NBSIR 83-27U4 IUSAR)

\title{
Initial Graphics Exchange Specification Test Library, Version 1.3
}

U.S. DEPARTMENT OF COMNERCE

National Bureau of Standards

National Engineering Laboratory

Center for Manufacturing Engineering

Automated Production Technology Division

Washington, DC 20234

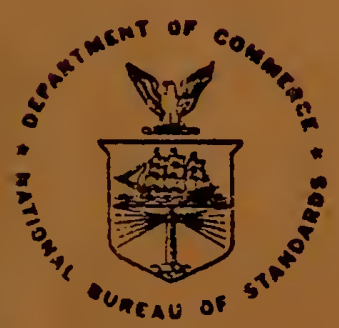

L.S. DEPARTMENT OF COMMERCE

NATIONAL BUAEAU OF STANDARDS

100

.056

$83-2704$

1983 


\section{INITIAL GRAPHICS EXCHANGE} SPECIFICATION TEST LIBRARY, VERSION 1.3

Bradford Smith. NBS

Michael Liewald. Boeing Commercial Airplane Co.

IGES Test, Evaluate \& Support Committee

U.S. DEPARTMENT OF COMMERCE

National Bureau of Standards

National Engineering Laboratory

Center for Manufacturing Engineering

Automated Production Technology Division

Wasnington. DC 20234 



\section{TABLE OF CONTENTS}

1.0 INTRODUCTION

$1-1$

2.0 TEST METHODOLOGY $2-1$

2.1 Test Ojectives 2-1

2.2 Test Procedure 2-1

2.3 Test Case Generation . 2-4

2.4 Testing Limitations 2-6

2.4.1 Validation of Test Cases 2-6

2.4.2 Completeness 2-6

2.4.3 Figures 2-7

2.4.4 Attributes 2-7

2.4.5 Compound Test 2-8

2.4.6 Numeric Accuracy 2-8

2.4.7 System Incompatibility 2-9

3.0 TEST LIBRARY STRUCTURE $3-1$

3.1 Entity Set 3-2

3.2 Entity Attributes $3-3$

3.3 Test Cases $3.1-1$

3.3.1 Circular Arc 3.1-1

3.3.2 Composite Entity $3.2-1$

3.3.3 Conic 3.3-1

3.3.4 Copious Data Entity $3.4-1$

3.3.5 Line $3.5-1$

3.3.6 Parametric Spline $3.6-1$

3.3.7 Point 3.7-1

3.3.8 Transformation Matrix 3.3-1

3.3.9 Angular Dimension 3.9-1

3.3.10 Diameter Dimension 3.10-1

3.3.11 General Label 3.11-1

3.3.12 General Note 3.12-1

3.3.13 Linear Dimension 3.13-1

3.3.14 Radius Dimension 3.14-1

3.3.15 Leader 3.15-1 


\section{TABLE OF CONTENTS (Continued)}

Page

3.3.16 Section

$3.16-1$

3.3.17 Witness Line

$3.17-1$

3.3.18 Associativity Instance

$3.18-1$

3.3.19 Line Fonts

$3.19-1$

3.3.20 Levels

$3.20-1$

3.3.21 BLANK Status

$3.21-1$

3.3.22 Labels/Subscripts

$3.22-1$ 


\section{LIST OF FIGURES}

Figure

Page

2.2-1 - Simple Test Flow

$2-3$

2.3-1 - Data Flow

$2-5$

3.3.1-1 - Circular Arc Cases 1 and 2 (with annotation)

$3.1-2$

3.3.1-2 - Circular Arc Cases 1 and 2 (actual part)

$3.1-3$

3.3.1-3 - Circular Arc Cases 3 and 4 (with annotation)

$3.1-6$

3.3.1-4 - Circular Arc Cases 3 and 4 (actual part)

$3.1-7$

3.3.2-1 - Composite Entity Case 1 (with annotation)

$3.2-2$

3.3.2-2 - Composite Entity Case 1 (actual part)

$3.2-3$

3.3.3-1 - Conic Cases 1 and 2 (with annotation)

$3.3-2$

3.3.3-2 - Conic Cases 1 and 2 (actual part)

$3.3-3$

3.3.4-1 - Linear String Form 11 (actual part)

$3.4-2$

3.3.4-2 - Linear String Form 12 (actual part)

$3.4-3$

3.3.4-3 - Linear String Form 13 (actual part)

$3.4-4$

3.3.5-1 - Line Cases 1 and 2 (with annotation)

$3.5-2$

3.3.5-2 - Line Cases 1 and 2 (actual part)

$3.5-3$

3.3.5-3 - Line Cases 3 and 4 (with annotation) 3.5-8

3.3.5-4 - Line Cases 3 and 4 (actual part)

$3.5-9$

3.3.5-5 - Line Cases 3 and 4 (magnified to show small lines)

$3.5-10$

3.3.6-1 - 2D Cubic Spline (aciual part)

$3.6-2$

3.3.6-2 - 3D Cubic Spline (actual part)

$3.6-3$

3.3.6-3 - Wilson-Fowler Spline (actual part)

$3.6-4$

3.3.6-4 - Modified Wilson-Fowler Splines (actual part)

$3.6-5$

3.3.6-5 - Uniform 3-Spline (actual part)

$3.6-6$

3.3.7-1 - Point Case 1 (actual part)

$3.7-2$

3.3.9-1 - Angular Dimension Cases 1 and 2 (with annotation)

$3.9-2$

3.3.9-2 - Angular Dimension Cases 1 and 2 (actual part)

$3.9-3$

3.3.9-3 - Angular Dimension Cases 1 and 2 (lef: side magnified)

$3.9-4$

3.3.9-4 - Angular Dimension Cases 1 and 2 (right side magnified)

$3.9-5$

3.3.9-5 - Angular Dimension Cases 3 and 4 (with lines) 3.9-23

3.3.9-6 - Angular Dimension Cases 3 and 4 (actual part) 3.9-29

3.3.10-1 - Diameter Dimension Cases 1 and 2 (with annotation) 3.10-2

3.3.10-2 - Diameter Dimension Cases 1 and 2 (actual part) 3.10-3

3.3.11-1 - General Label Cases 1 and 2 (with annotation) 3.11-2 


\section{LIST OF FIGURES (Continued)}

Figure

Page

3.3.11-2 - General Label Cases 1 and 2 (actual part)

$3.11-3$

3.3.11-3 - General Label Cases 3 and 4 (with annotation)

$3.11-8$

3.3.11-4 - General Label Cases 3 and 4 (actual part)

$3.11-9$

3.3.12-1 - General Note Cases 1 and 2 (actual part)

$3.12-2$

3.3.12-2 - General Note Cases 3 and 4 (actual part)

$3.12-5$

3.3.12-3 - General Note Cases 3 and 4 (rotated text magnified)

$3.12-6$

3.3.12-4 - General Note Cases 3 and 4 (long text magnified)

$3.12-7$

3.3.12-5 - General Note Cases 3 and 4 (small text magnified)

$3.12-8$

3.3.13-1 - Linear Dimension - Cases 1 and 2 (with annotation)

3.13-2

3.3.13-2 - Linear Dimension Cases 1 and 2 (actual part)

$3.13-3$

3.3.13-3 - Linear Dimension Cases 1 and 2 (left side magnified)

$3.13-4$

3.3.13-4 - Linear Dimension Cases 1 and 2 (right side magnified)

$3.13-5$

3.3.14-1 - Radius Dimension Cases 1 and 2 (with annotation)

$3.14-2$

3.3.14-2 - Radius Dimension Çases 1 and 2 (actual part)

3.14-3

3.3.15-1 - Leader - Case 1 (actual part)

3.15-2

3.3.18-1 - Associativity Instance Case 1 (actual part)

3.18-2

3.3.18-2 - Associativity Instance Case 2 (actual part)

3.18-3

3.3.19-1 - Examples of Line Fonts

$3.19-2$

3.3.20-1 - Level Case 1 (with annotation and all levels visible)

3.20-2

3.3.20-2 - Level Case I (actual part with all levels visible)

$3.20-3$

3.3.21-1 - Blank Status Case 1 (actual entities are blanked)

3.21-2

3.3.22-1 - Label/Subscript Case 1 (with annotation)

3.22-2

3.3.22-2 - Label/Subscript Case 2 (actual part) 
IGES Test. Evaluate and SupDort Committee

IGES Chairman: Brad Smith, National Bureau of Standards, Washington, D.C.

IGES Coordinator: Joan Wellington, National Bureau of Standards, Washington, D.C.,

Test, Evaluate, Mike Liewald, Boeing Commerical Airplane Company, Seattle, and Support Chairman: Washington

Library Tecinnical Coordinator:

David Briggs, Boeing Commercial Airplane Company, Seattle, Washington

Committee Members:

Robert Ard, Structural Dynamics Research Corp., Millford, Ohio

Peter Benjamin, Lockheed Missiles and Space Co., Sunnyvaie, California

Istvan Bodnar, Control Data, Arden Hills, Minnesota

Noel Christensen, Bendix Corp., Kansas City, Missouri

Dave Ellis, Racal-Redac, Holliston, Massachusetts

Roger Gale, General Dynamics, Pomona, California

Albert Gibbons, Westington Electric Corp., Pirtsburgh, Pennsylvania

William Gruttke, McDonnell Douglas Automation Company, St. Louis, Missouri

Douglas Hakala, Manufacturing Data Systems Inc., Ann Arbor, Michigan

Jack Horgan, Applicon Corp., Burlington, Massachusetts

Robert Ivey, Westinghouse Electric Corp., Monroeville, Pennsylvania

Srephen Kaminski, Hughes Aircraft, Culver City, California

J.C. Kelly, Sandia National Laboratories, Albuquerque, New Mexico

Philip Kennicott, General Electric Co., Scheneciady, New York

Andrew Pauker, Computervision Corp., Bedford, Massachusetts

Mark Quinlan, CALMA, San Diego, California

Patrick Rourke, Newport News Shipbuilding, Newport News, Virginia

Paul Serednicky, IBM, Poughkeepsie, New York

Robert Wolf, Xerox, Webster, New York 
- 
This document contains a library of benchmark tests to be used to verify the interface capability possible with the Initial Graphics Exchange Specification (IGES) (Reference A). IGES provides a common data format to facilitate the exchange of data between different Computer Aided Design/Computer Aided Manufacturing (CAD/CAM) systems.

The test cases outlined in this document provide data for software modules which translate IGES format to and from the format of a particular CAD/CAM system. The geometric and drafting entities contained in these parts comprise only a limited portion of all IGES entities and are intended to demonstrate some capabilities of an IGES translator on an individual entity basis. This set of tests does not constitute a test of compliance with any existing or proposed standardization of IGES. Subsequent tests with more complex parts should be made to insure the suitability of the processors for a user's production environment.

The library has been prepared by the IGES Test, Evaluate, and Support Committee and has been iterated through several versicns during its development. Test Library Versions $1.0,1.1$, and 1.2 were made available only to Test, Evaluate, and Support Committee members for verification of accuracy and to evaluate the utility of individual cases. During this process, many cases and the associated documentation were modified to addiess errors, make the case more useful, or clarify the supporting documentation. Version 1.3 represents the results of this effort, and is the first version of the Test Library to be made available to the general community. Future versions will incorporate additional cases and address any reported errors.

While the Test, Evaluate, and Support Committee has attempted to remove all errors from this library, no responsibility or liability is accepted by IGES, by members of IGES committees, or by firms represented in IGES for decisions made on the basis of the use of these test cases. 
The objective of this series of tests is to demonstrate the ability of CAD/CA.M systems to accept data in IGES format and transform the data into a format compatible with the receiving CAD/CAM system without significant loss of data accuracy or design intent.

The intended users of IGES span a wide range of applications, each having differing requirements on the data retrieved from IGES format. Each application's requirements will determine what constitutes acceptable results from these tests. Due to a wide range of requirements, no acceptance criteria are given and it is left to each user to determine if the results of these tests are acceptable in his environment.

Each entity listed in Section 3.0 will be tested individually and in combination with other entities to verify the ability of the CAD/CAM system to process the IGES entity. Each test case will contain a variety of examples of the entity such as a wide range of coordinate values, rotations, intersecting entities, non-model definition spaces, various fonts, various text/arrow configurations, and complex sub-entity arrangements.

\subsection{TEST PROCEDURE}

While IGES does not endorse any explicit testing and evaluation procedures, the parts in this library are intended as input to a software module which translates IGES format to the format of a particular CAD/CAM system. The output of this module will be a set of parts in the native format of the receiving system. In order to evaluate the conversion, the user will have to develop evaluation techniques and acceptance criteria suited to the user's needs. This criteria may include a combination of usability and functionality of the entities created, retention of design intent, visual display, and numeric comparison of parameter data. None of these by themselves is, in general, a complete or accurate evaluation criterion. Different users will have differing circumstances in which IGES will be utilized. These may 
range from a single pre-processor and post-processor to several of each. Figure 2.2-1 illustrates a very simple test flow which transfers a test case once through a post-processor and once through a pre-processor. Evaluators must decide for themselves at what points evaluation will be performed. They must also decide whether they will evaluate upon a single pass or multiple loops through the pre- and post-processors. Users having more than one pre- and/or post-processor will have more than one route possible and must make their own selection of routes to evaluate. The visual and IGES formatted representations contained in this document are not "master definitions" which other representations should be compared against. Valid representations of the data in the test parts may be generated which differ substantially in parameterization from the examples in this document. 


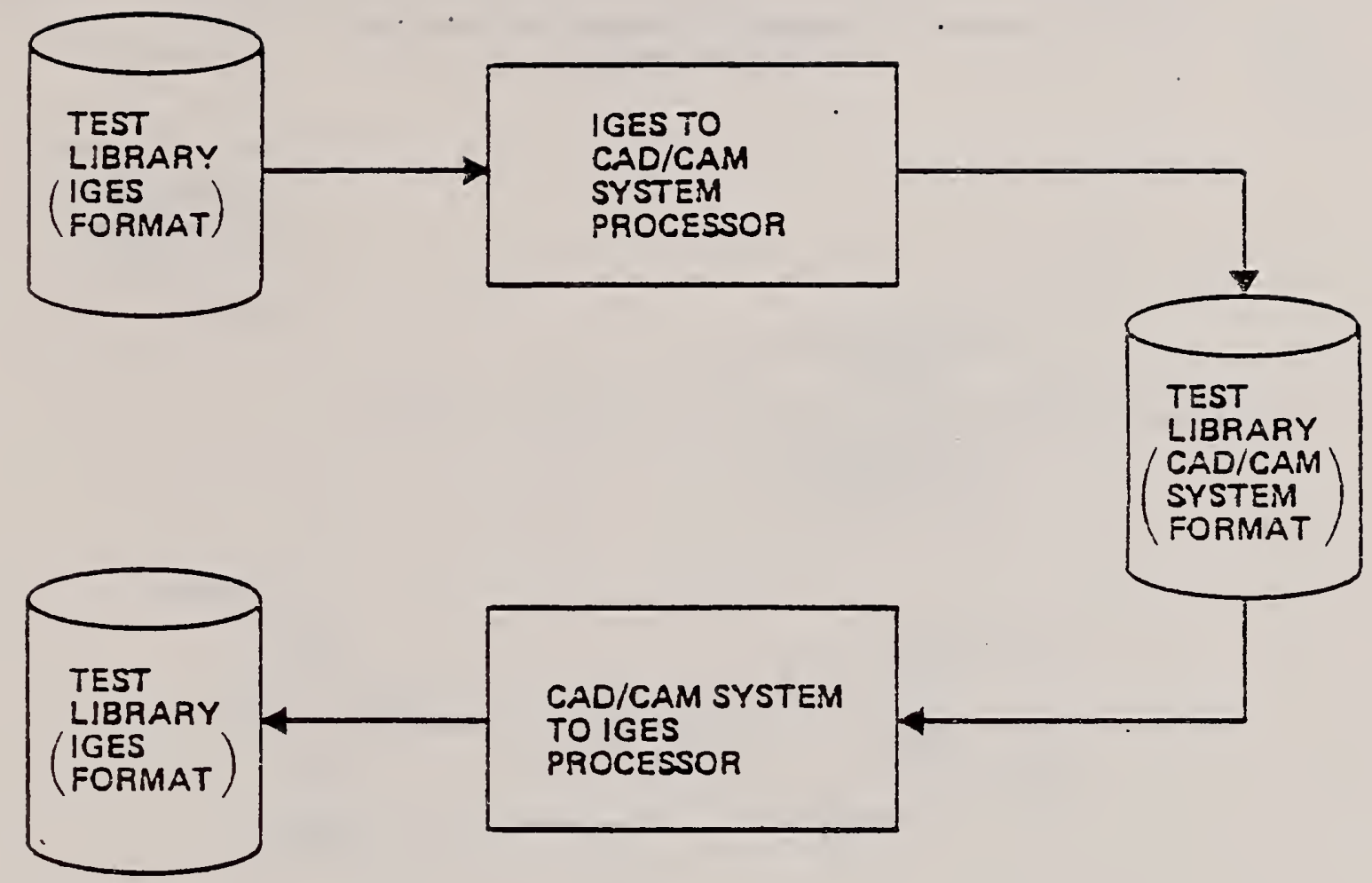

Figure 2.2-1 - Simple Test Flow 
The majority of these test cases were developed within the Boeing CAD/CAM integrated Information Network (CIN) (Reference C) environment and transformed into IGES format through an IGES/CIIN postprocessor. Additional cases were provided by participating CAD/CAM system developers. Figure 2.3-1 shows the data flow. 


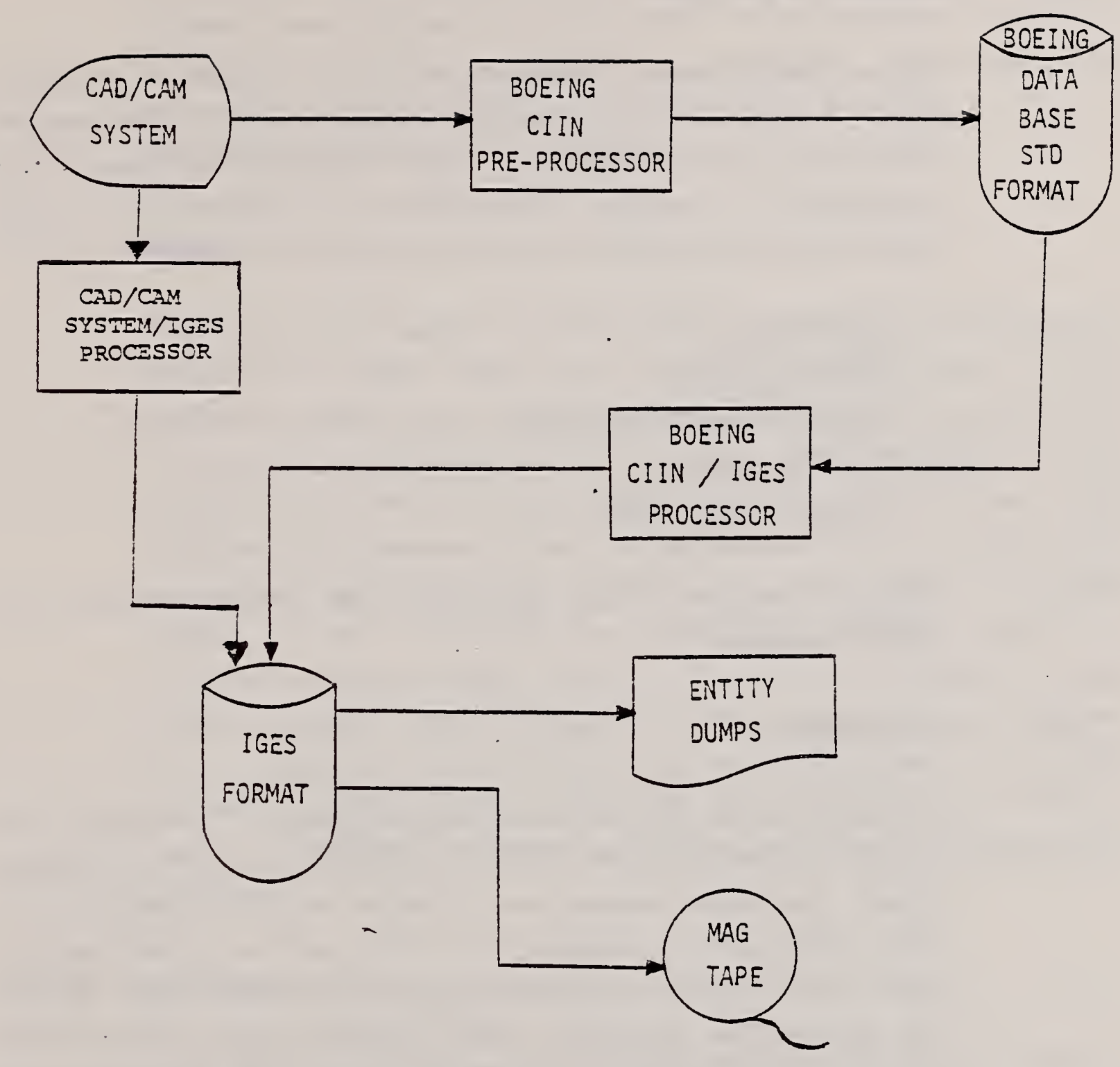

Figure 2.3-1 - Data Flow 


\title{
2.4.1 Validation of Test Cases
}

The test cases presented in this document have themselves on the date of issuance received only limited testing. Despite the efforts of the preparers and reviews by Test, Evaluate, and Support Committee undetected errors may persist in the test cases. Only extensive use of these files will reveal such errors. If any problems are found, they should be reported to

\author{
Joan Wellington \\ IGES Coordinator \\ National Bureau of Standards \\ A-353, Building 220 \\ Washington, D.C. 20234
}

Subsequent versions of this test library will attempt to correct any reported problems.

\subsubsection{Completeness}

This version of the IGES test library does not attempt to exhaustively test all IGES entities, attributes; associativities, properties, and combinations thereof. Each case addresses only a limited set of functionality and is largely independent of the other cases. The tests are rather general in nature and do not have any particular application thrust. In later versions this library will be extended to cover additional entities and attributes. Moreover, the test cases in these future versions will be organized into groups appropriate to the more common application areas. The inclusion or exclusion of a particular entity or attribute should not be interpreted as a statement of its relative usefulness. No attempt has been or will be made to establish a priority scheme in this area.

The initial set of test cases has been provided by a small number of sources. This may have caused some unintentional bias to be introduced into the test cases. This would be reflected not only in the specific 
features chosen for testing but also the manner in which the features are tested. Even subtle choices such as range of coordinate values and viewing orientation can inadvertently affect the test cases. The software used to generate these files uses a limited range of numeric representations (i.e., in number of decimal places), of global parameter values, and in the sequencing of data. Further tests with data from a variety of different sources constitutes a more complete test.

\subsubsection{Figures}

The figures which appear in this document were derived from hardcopy taken from the CRT screen of the originating graphics system. These figures were appropriately scaled for viewing clarity and are therefore not a one-to-one representation of the data file. The appearance of small details such as line font pattern which appear in the file may have been distorted by this procedure. Even the appearance of actual geometry may be distorted as when relatively large and small items appear in the same figure. In order to reproduce these figures on the graphics screen of a CAD/CAM system processing these test cases it may be necessary to issue several viewing commands to achieve the same scale and viewing orientation. A simple visual comparison is not sufficient to accurately verify successful transfer of data. The intent of the figures is to provide a visually recognizable description of the test cases, not a "template" for evaluation.

\subsubsection{Attributes}

Some IGES attributes are not defined in geometric detail but rather in terms of the default values of the active CAD/CAM system. For example the line font parameter has value 1, 2, 3, or 4 for solid, dashed, phantom, and centerline font respectively. The spacing and line pattern for the last three forms may vary from graphics system to graphics system and are not defined in the IGES specification. Therefore some changes in the appearance of line font may be introduced when transferring from system $\lambda$ to system $B$ but such a change would not be due to a processor error. Other attributes which fall into this category include line weight and tex: 
font. The figures which appear in the document were generated on a specific CAD/CAM system. This should not be interpreted as an endorsement by IGES of that specific implementation of these attributes.

\subsubsection{Compound Test}

Even though the test cases presented in this document are limited in scope, each case addresses multiple functionality. For example the transier of a geometric entity is frequently tied with its cosmetic appearance e.g., line font. In the majority of cases, the multiplicity is limited to an entity and its attributes. Careful examination of the output of an IGES processor is required to determine whether all aspects of the test case have been successiully transferred. Conversely a cursory or simple visual examination of output may lead to erroneous conclusions on completeness of data transfer. It is up to each individual user to decide the relative importance of each feature.

\subsubsection{Numeric Accuracy}

The internal representation of geometric entities in the various CAD/CAM systems differ greatly among themselves and also from the IGES representation. Therefore in transferring data to or from IGES format, calculations must be performed on the source representation in order to achieve the target representation. In the course of this procedure some computational inaccuracies may be introduced. Parameters which identically match in one representation may differ slightly in a second representation. Parameters with zero value in one representation may have small non-zero values in another representation. Within the parameter data of some entities in the test library, two values intended to be identical may differ by some small amount. For example, the start and terminate points of a curve intended to be closed may not have identical cocrdinate values. Further, these small representational inaccuracies may lead to boundary points for mathematical curves that do not lie exactly on those curves. Sophisticated numerical analysis techniques may be required to reduce the adverse effects of this problem. Careful examination of the output of IGES processors is required to detect such problems and even more detailed 
analysis is needed to determine the point where the error may have been introduced.

\subsubsection{System Incompatibilities}

There is a significant amount of overlap of functionality provided by the various CAD/CAM systems. However, the user interfaces and internal data representation employed to support this common functionality difier greatly among these systems and also from IGES. A given graphics system may provide perfectly acceptable functionality to its user community without having a direct correspondence to each and every IGES entity, property, and associativity. An obvious example would be an electronics system which did not support ruled and parametric spline surfaces. Conversely data which does reside in a given graphics system may have no corresponding entity, property, or associativity in IGES to directly map into. The inclusion or exclusion of a specific data type in the specifications should not be interpreted as a statement by IGES on its relative merits.

When an IGES pre-processor encounters an entity in the native data base which has no equivalent IGES entity, it has two basic choices. It can ignore the information or convert it to one or more IGES entities. The userdefined IGES entities (macro, associativity and property) will be useful in this context.

When an IGES post-processor encounters an entity in the IGES file which has no equivalent entity in its native data base, it has two basic choices. It can either discard the data or store it in an "equivalent" form in the target system. There are no hard and fast IGES rules for cetermining what is an appropriate "equivalent" representation. It is up to each user to determine whether data has been adequately captured in the target system. It is also a subjective judgment whether any loss of data or transformation of data is acceptabie in a given user environment.

A user may minimize this type of problem by eliminating or restricting the usage of features and aata structures in one system which have little or no 
correspondence in a second system into which it is intended to transfer data via IGES. Future extensions to IGES will also help alleviate this type of problem. 
For each entity type, one or more test cases are provided:

- Case 1 contains multiple occurrences of the entity in a definition space which coincides with model space (XYZ). Several different variations of the entity are provided.

- Case 2 is identical to the first except the entities are in a nonmodel definition space (XT, YT, ZT).

- Case 3 contains unusual or extreme examples of the entity.

- Case 4 is identical to the third case except it is in a non-modei definition space.

For each entity a figure for the test case with descriptive annotation is given along with a figure of the actual test case. The descriptive annotation is not part of the test case and will not be present in the IGES file.

The following conventions are followed for the test cases:

- Test cases contain only the entities being tested

- Test cases contain values (ccordinates and coefficients) with magnitudes from 0 to 1000 except for the point case where larger magnitudes were used

- All transformation matrices have zero translation components

- All text originates from Computervision CADDS-3 Rev. 10.020V6B

- No provision made for properties

The test cases contain geometric and drafting entities defined in an appropriate coordinate system, but do not include the IGES Drawing or View entities. All figures in this document have a viewing orientation corresponding to looking along the positive $Z T$ axis toward the origin of the unrotated XT-YT plane (right-handed coordinate system). Thus the figures for the model and non-model definition space cases are identical when viewed from the appropriate orientation. The regeneration of the 
appropriate orientation is leit to the user interface of the particular system using, if necessary, the orientation information given with the case description. This information consists of a series of rotations which will move the model relative to the user's viewing perspective. The rotations are described by an ordered triple $(A, B, C)$ where the values are rotation angles measured in degrees. For example, an orientation of $\left(30^{\circ}, 60^{\circ}, 90^{\circ}\right)$ means the user must enter the commands to rotate the model relative to the user's viewing perspective as follows:

- Begin with a viewing orientation corresponding to looking along the positive $Z$ axis toward the origin of the unrotated $X-Y$ plane (righthanded coordinate system).

- First, $30^{\circ}$ top out, bottom in

- Second, $60^{\circ}$ left out, right in

- Third, $90^{\circ}$ counter clockwise

These orientation descriptions are for use as an aid to the user in reaching a viewing orientation similar to the figure in the document and do not supercede the IGES transformation matrix entity.

\subsection{ENTITY SET}

The following IGES geometric entities are contained in this library:

- Circular Arc (entity $¥ 100)$

- Composite Entity (entity 非102)

- Conic (entity 非104) FORM 1, 2, 3

- Copious Data Entity (entity \#1106) FORM 11, 12, 13, 40

- Line (entity $\mid ⿰ 110)$

- Parametric Spline (entity 非12)

Cubic Splines

Wilson Fowler Splines

Modified Wilson Fowler Splines 


$$
\begin{aligned}
& \text { B-spline } \\
& \text { - Point (entity \#116) } \\
& \text { - Transformation Matrix (entity \#124) }
\end{aligned}
$$

The following IGES annotation entities are contained:

- Angular Dimension (entity 作202)

- Diameter Dimension (entity 非206)

- Ceneral Label (entity 非10)

- General Note (entity 非12)

- Leader (entity 非14)

- Linear Dimension (entity \#216)

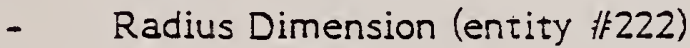

The IGES structural entity Associativity Instance (FORM ik 1 ) is also contained.

Section 3.3 describes the individual tests to be performed. For each test an explanation of the test case and a corresponding IGES formatted definition are given.

\subsection{ENTITY ATTRIBUTES}

The following IGES features (attributes) are contained in this library:

- Line Fonts

- Levels

- Entity Labeis/Subscript

- Blanked Status 


\section{-}


NOTE: Some test cases contain zero values in the DE Reserved fields (16 and 17)

3.3.1 Circular Arc

- Case 1 (Figures 3.3.1-1 and 3.3.1-2) tests the following items:

- Full circles

- Fonted circles (solid, dashed, phantom, centerline)

- Arcs

- $\quad$ Case 2

- Identical to Case 1 except non-model space is used with rotation $\left(30^{\circ}, 60^{\circ}, 90^{\circ}\right)$ per section 3.0

- Case 3 (Figures $3.3 .1-3$ and 3.3.1-4) tests the following:

- Large circle (diameter $=1000 \mathrm{in}$ )

- Small circle (diameter $=0.01$ in)

- Case 4

- Identical to Case 3 except non-model space is used with rotation $\left(60^{\circ}, 120^{\circ},-60^{\circ}\right)$ per section 3.0

NOTE: Circle entities that appear closed may have start and terminate points which are not identica! but which differ by $10^{4}$ 
FONTED

FULL CIRCLES
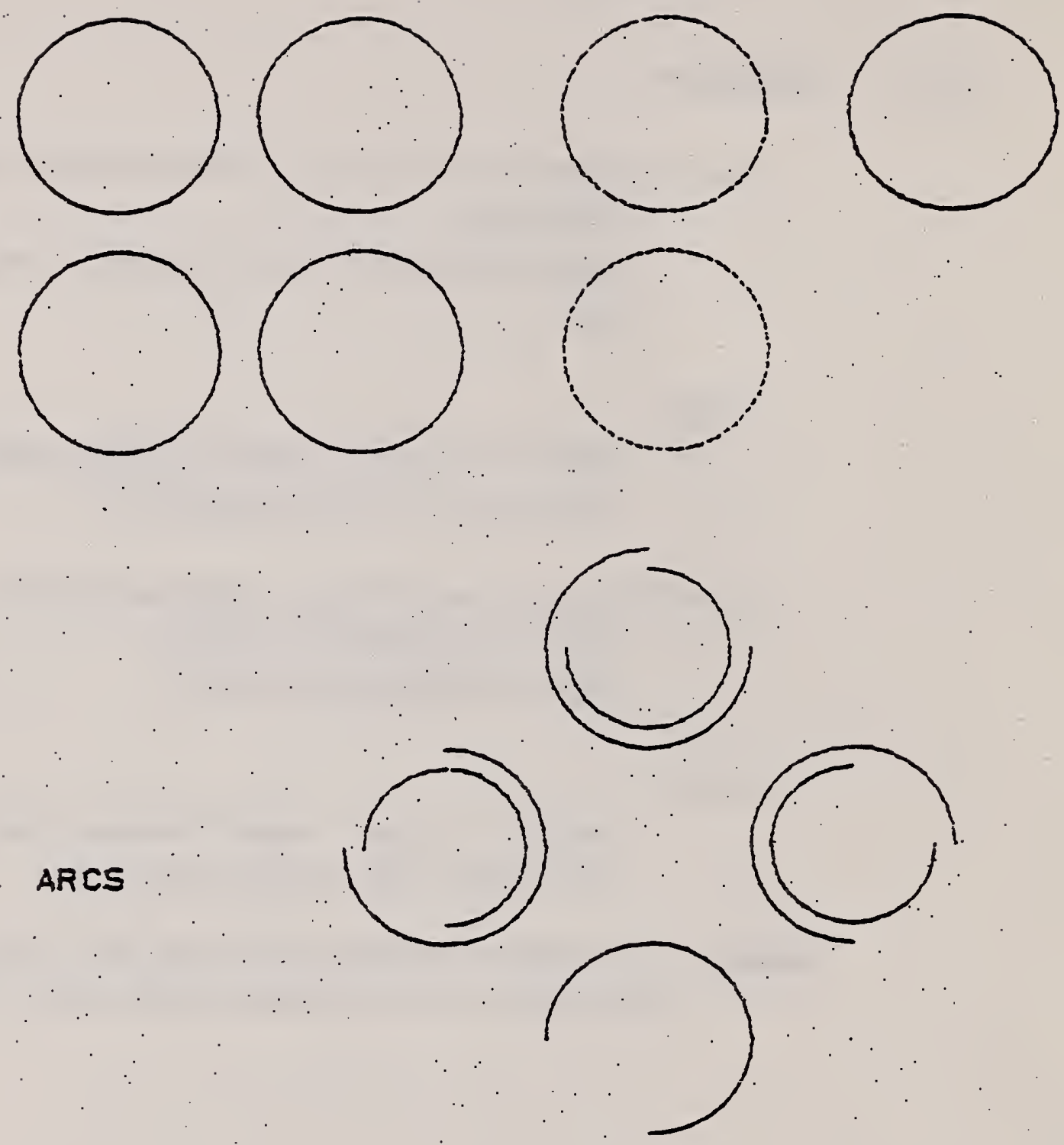

Figure 3.3.1-1 Circular Arc Cases 1 and 2 (with annotation)

Case 1: Rotation $\left(0^{\circ}, 0^{\circ}, 0^{\circ}\right)$

Case 2: Rotation $\left(30^{\circ}, 60^{\circ}, 90^{\circ}\right)$ 

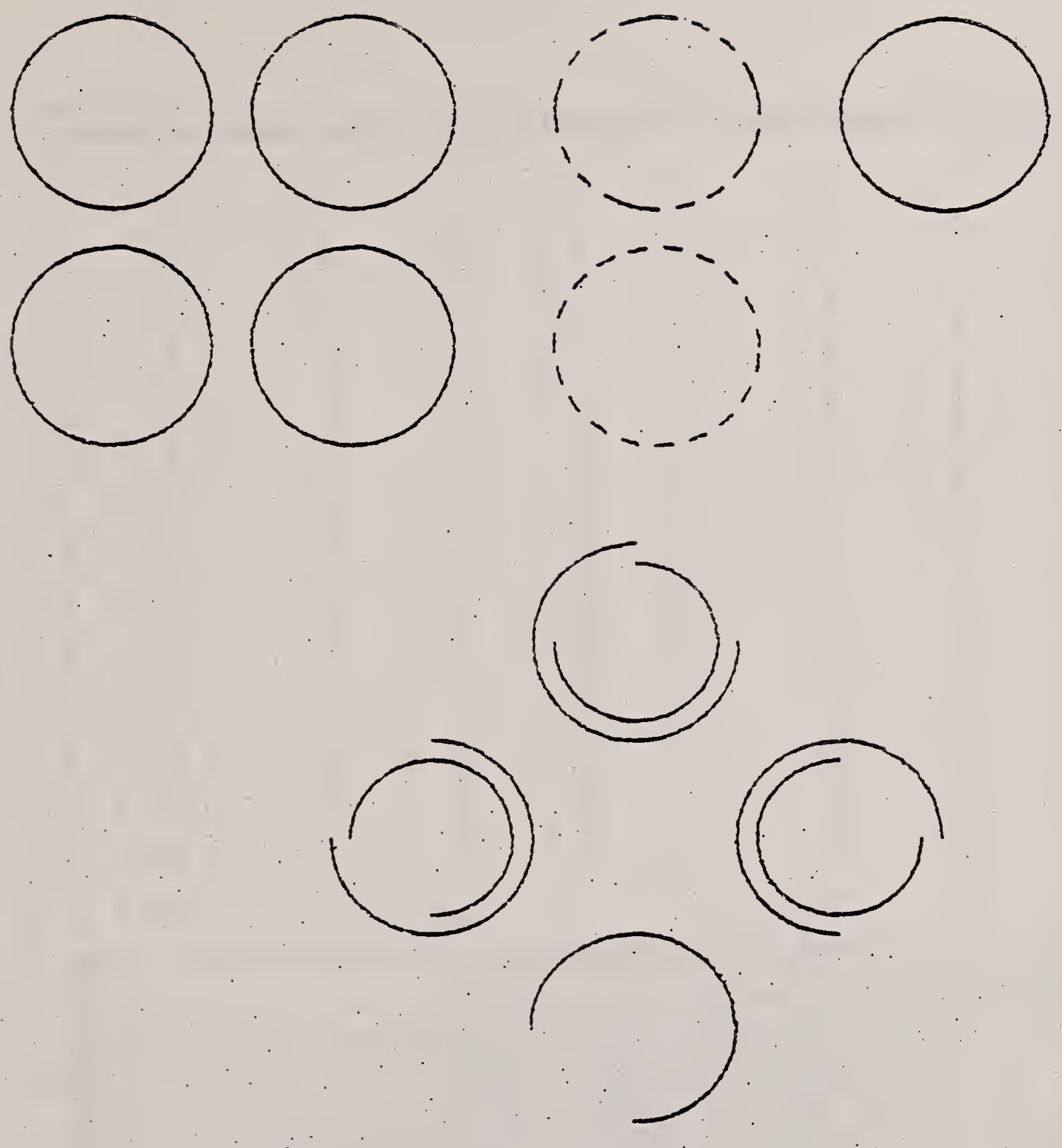

Figure 3.3.1-2 Circular Arc Cases 1 and 2 (actual part) 


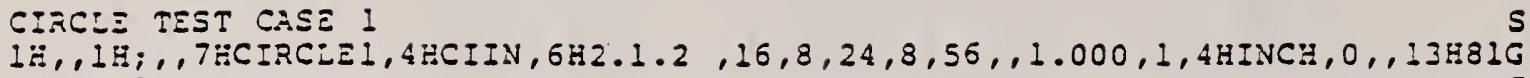

$0714.092244, \ldots$, ;

124

124

100

100

100

100

100

100

100

100

100

100

100

100

100

100

100

100

100

100

100

100

100

100

100

100

100

100

100

100

$\begin{array}{rl}1 & 1 \\ 0 & 0 \\ 2 & 1 \\ 0 & 0 \\ 3 & 1 \\ 0 & 0 \\ 4 & 1 \\ 0 & 0 \\ 5 & 1 \\ 0 & 0 \\ 6 & 1 \\ 0 & 0 \\ 7 & 1 \\ 0 & 0 \\ 8 & 1 \\ 0 & 0 \\ 9 & 1 \\ 0 & 0 \\ 10 & 1 \\ 0 & 0 \\ 11 & 1 \\ 0 & 0 \\ 12 & 1 \\ 0 & 0 \\ 13 & 1 \\ 0 & 0 \\ 14 & 1 \\ 10 & 0 \\ 15 & 1 \\ 0 & 0\end{array}$

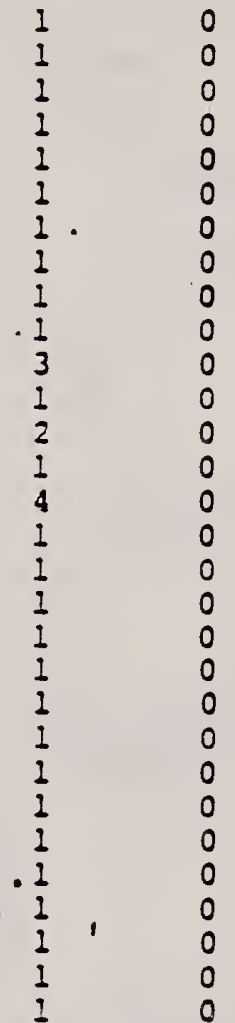

$$
\begin{aligned}
& 0 \\
& 0 \\
& 0 \\
& 0 \\
& 0 \\
& 0 \\
& 0 \\
& 0 \\
& 0 \\
& 0 \\
& 0 \\
& 0 \\
& 0 \\
& 0 \\
& 0 \\
& 0 \\
& 0 \\
& 0 \\
& 0 \\
& 0 \\
& 0 \\
& 0 \\
& 0 \\
& 0 \\
& 0 \\
& 0 \\
& 0 \\
& 0 \\
& 0 \\
& 0
\end{aligned}
$$

$\begin{array}{lllll}0 & 0 & 0 & 0 & D^{6} \\ 0 & 0 & 0 & 0\end{array}$

$\begin{array}{lllll}0 & 0 & 0 & 0 & D\end{array}$

$\begin{array}{llllll}0 & 0 & 0 & 0 & D\end{array}$

$\begin{array}{llllll}0 & 0 & 0 & 0 & 0 & 0\end{array}$

$\begin{array}{lllll}0 & 0 & 0 & 0 & 0\end{array}$

$\begin{array}{llllllll}0 & 0 & 0 & 0 & D\end{array}$

$\begin{array}{llllllllll}0 & 0 & 0 & 0 & D\end{array}$

$\begin{array}{llllll}0 & 0 & 0 & 0 & D\end{array}$

$\begin{array}{llllll}0 & 0 & 0 & 0 & D\end{array}$

00000

$\begin{array}{lllll}0 & 0 & 0 & 0 & 0\end{array}$

$\begin{array}{lllll}0 & 0 & 0 & 0\end{array}$

$\begin{array}{llllllllll}0 & 0 & 0 & 0 & 0\end{array}$

$\begin{array}{lllll}0 & 0 & 0 & 0 & 0\end{array}$

$O D$

$\begin{array}{llllll}0 & 0 & 0 & 0 & D\end{array}$

25

$\begin{array}{llllllll}0 & 0 & 0 & 0 & 0 & 25\end{array}$

$\begin{array}{llllllll}0 & 0 & 0 & 0 & 0 & 28\end{array}$

$\begin{array}{lllllll}0 & 0 & 0 & 0 & D & 29\end{array}$

$124,1.000,0.0,0.0,0.0,0.0,1.000,0.0,0.0,0.0,0.0,1.000,0.0,0,0 ;$

$100,0.0,6.000,6.000,11.000,6.000,11.000,6.000,0,0$;

$100,0.0,-6.000,6.000,-1.000,6.000,-1.000,6.000,0,0$;

$100,0.0,-6.000,-6.000,-1.000,-6.000,-1.000,-6.000,0,0$;

$100,0.0,6.000,-6.000,11.000,-6.000,11.000,-5.000,0,0$;

$100,0.0,21.000,6.000,26.000,6.000,26.000,6.000,0,0$;

$100,0.0,21.000,-6.000,26.000,-6.000,26.000,-5.000,0,0$;

$100,0.0,35.000,6.000,40.000,6.000,40.000,6.000,0,0$;

$100,0.0,30.000,-31.000,35.000,-31.000,30.000,-36.000,0,0$;

$100,0.0,20.000,-2 \$ .000,20.000,-16.000 ; 25.000,-21.000,0,0$;

$100,0.0,10.000,-31.000,5.000,-31.000,10.000,-25.000,0,0$;

$100,0.0,20.000,-41.000,20.000,-46.000,15.000,-41.000,0,0$;

$100,0.0,30.000,-31.000,30.000,-27.000,34.000,-31.000,0,0$;

$100,0.0,20.000,-21.000,16.000,-21.000,20.000,-17.000,0,0$;

$100,0.0,10.000,-31.000,10.000,-35.000,6.000,-31.000,0,0$;

5

iG

2D

$30 P$

15

$\begin{array}{rr}O D & 30 \\ 1 P & 1 \\ 3 P & 2 \\ 5 P & 3 \\ 5 P & 4 \\ 7 P & 4 \\ 9 P & 5 \\ 11 P & 6 \\ 13 P & 7 \\ 15 P & 8 \\ 17 P & 9 \\ 19 P & 10 \\ 21 P & 11 \\ 23 P & 12 \\ 25 P & 13 \\ 27 P & 14 \\ 29 P & 15 \\ 7 & 1\end{array}$


CERCEE TEST CASE 2

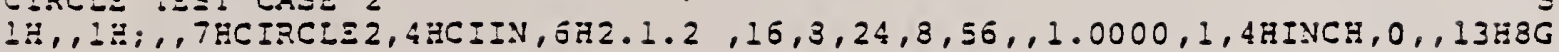

$10714.092709, \ldots 1 ;$

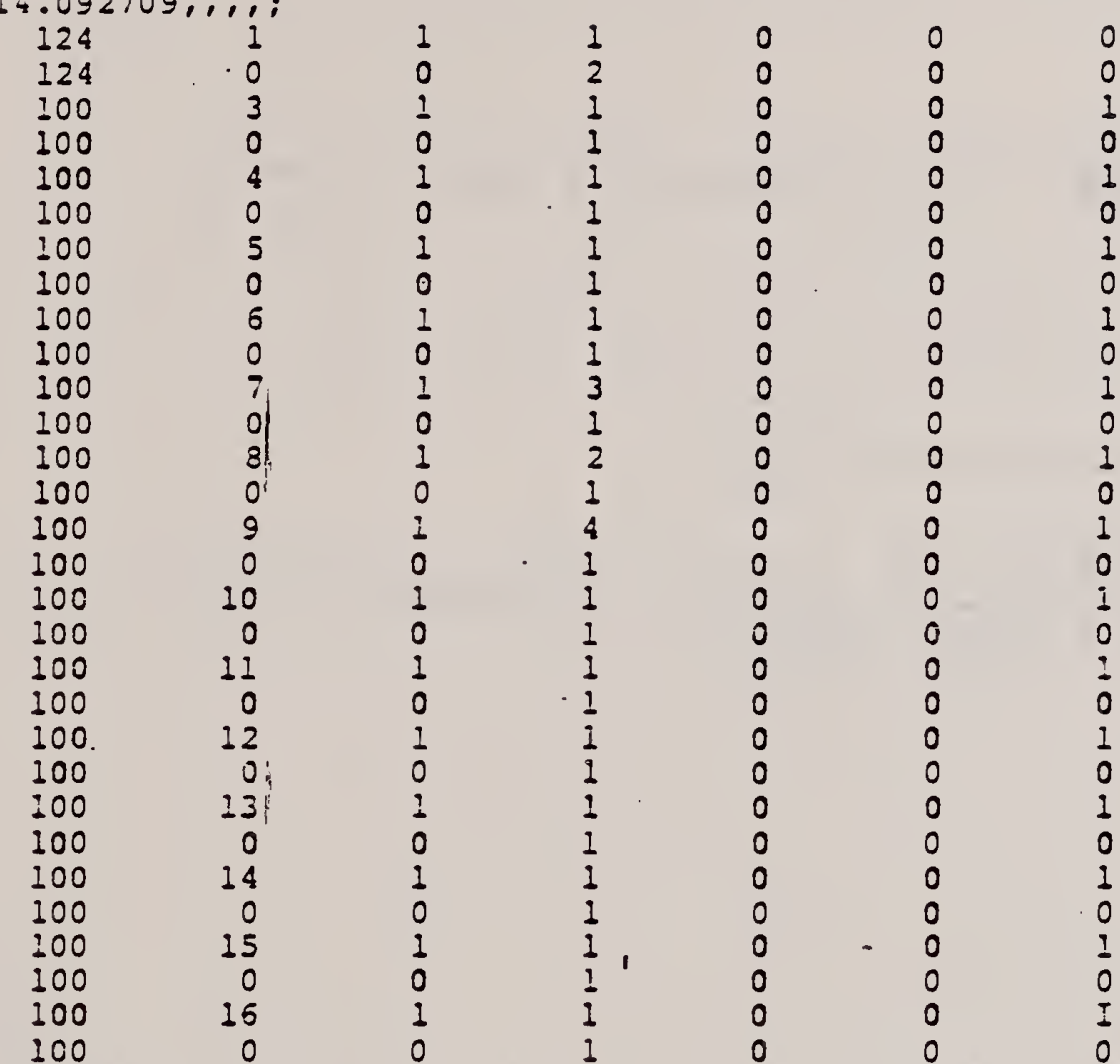

$124,0.0, .5000,-.8660,0.0,-.8660, .4330, .2500,0.0, .5000, .7500$, $.4330,0.0,0,0$;

$100,0.0,6.0000,6.0000,11.0000,6.0000,11.0000,6.0000,0,0$;

$100,0.0,-5.0000,6.0000,-1.0000,6.0000,-1.0000,6.0000,0,0$;

$100,0.0,-6.0000,-6.0000,-1.0000,-6.0000,-1.0000,-5.0000,0,0$

$100,0.0,6.0000,-6.0000,11.0000,-6.0000,11.0000,-5.0000,0,0$;

$100,0.0,21.0000,6.0000,26.0000,6.0000,25.0000,6.0000,0,0$;

$100,0.0,21.0000,-6.0000,25.0000,-5.0000,25.0000,-5.0000,0,0$;

$100,0.0,35.0000,5.0000, \div 0.0000,6.0000,40.0000,6.0000,0,0$

$100,0.0,30.0000,-31.0000,35.0000,-31.0000,30.0000,-36.0000,0,0$;

$100,0.0,20.0000,-21.0000,20.0000,-15.0000,25.0000,-21.0000,0.0$;

$100,0.0,10.0000,-31.0000,5.0000,-31.0000,10.0000,-25.0000,0,0$;

$100,0.0,20.0000,-41.0000,20.0000,-46.0000,15.0000,-41.0000,0,0$;

$100,0.0,30.0000,-31.0000,30.0000,-27.0000,34.0000,-31.0000,0,0$;

$100,0.0,20.0000,-21.0000,16.0000,-21.0000,20.0000,-17.0000,0,0$;

$100,0.0,10.0000,-31.0000,10.0000,-35.0000,6.0000,-32.0000,0,0$;

16

$2 D$

308

16

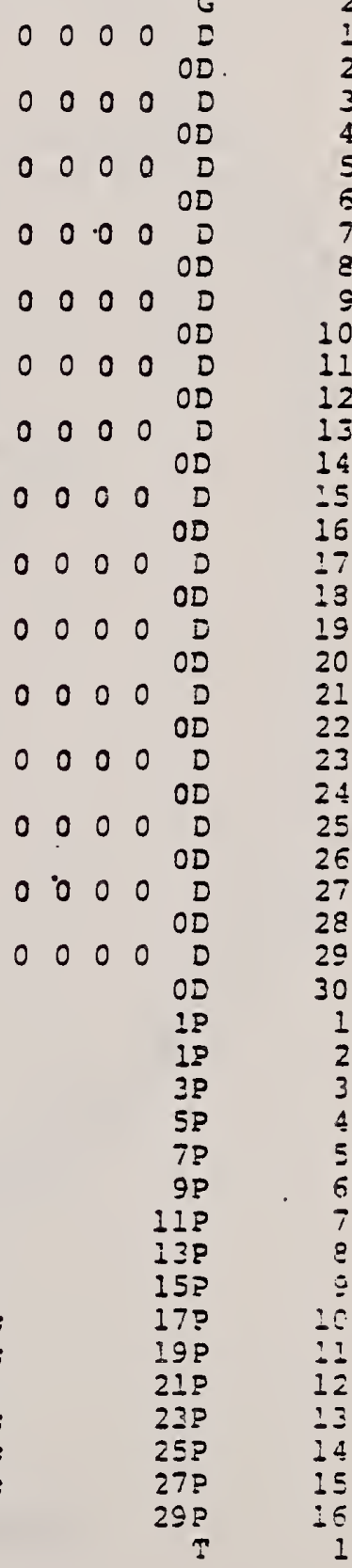




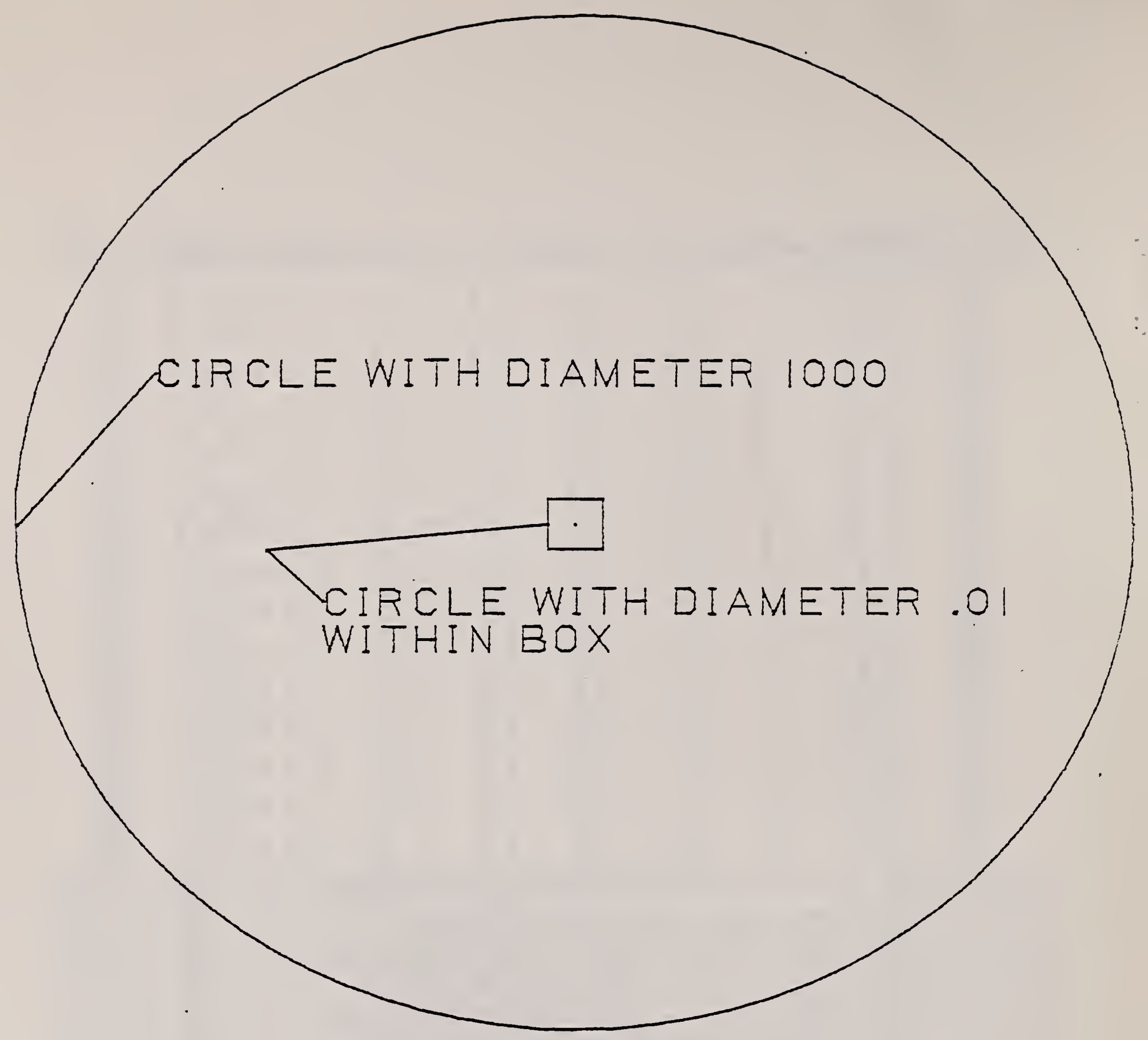

Figure 3.3.1-3 Circular Arc Cases 3 and 4 (with annotation)

Case 3: Rotation $\left(0^{\circ}, 0^{\circ}, 0^{\circ}\right)$

Case 4: Rotation $\left(60^{\circ}, 120^{\circ},-60^{\circ}\right)$ 


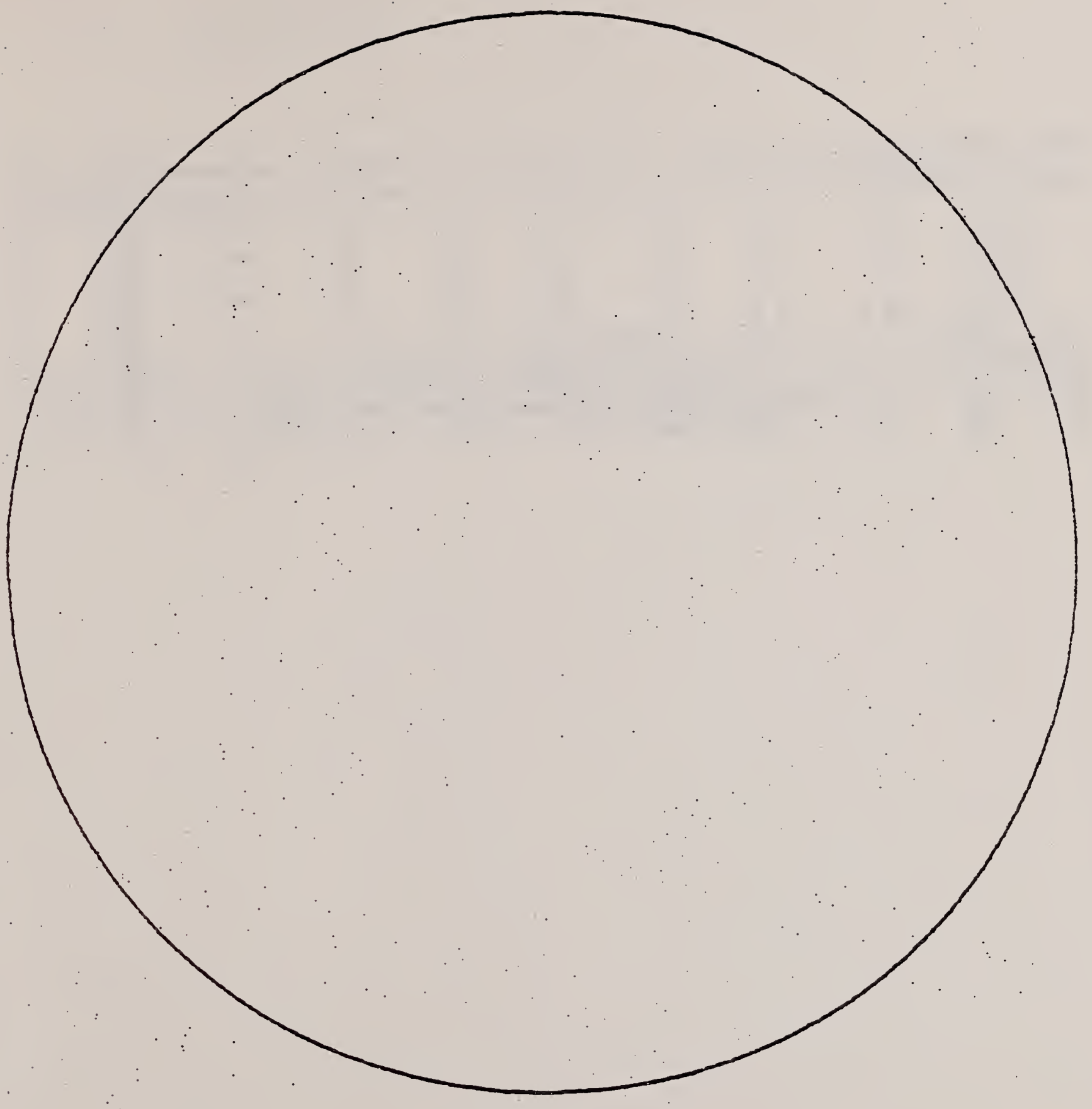

Figure 3.3.1-4 Circular Arc Cases 3 and 4 (actual part) 


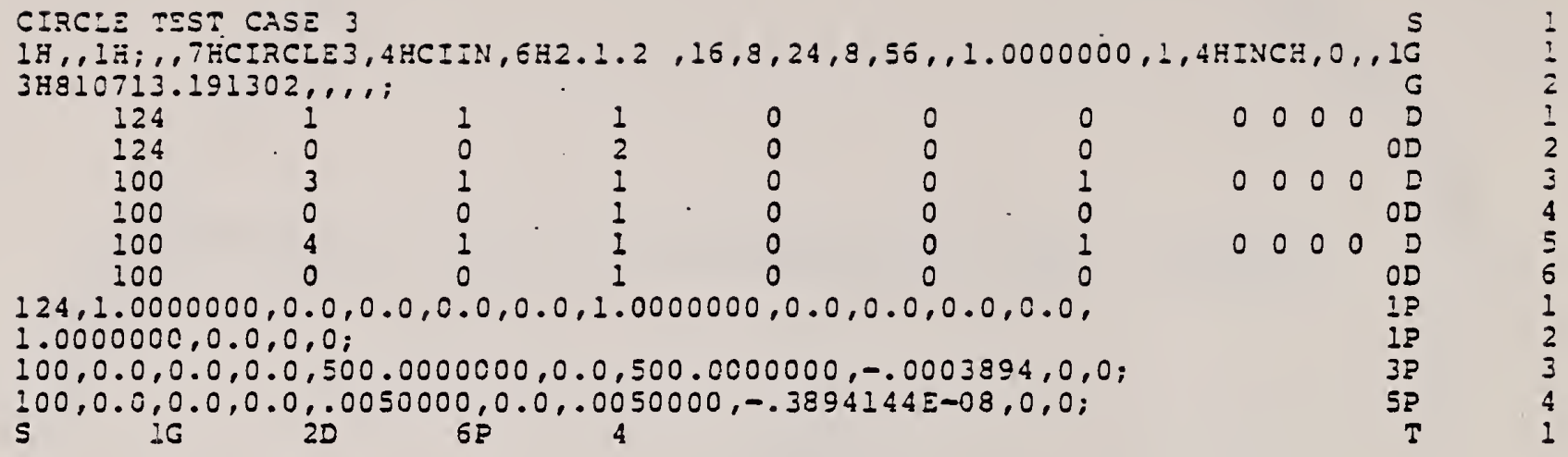


CIRCLE TEST CA.SE 4

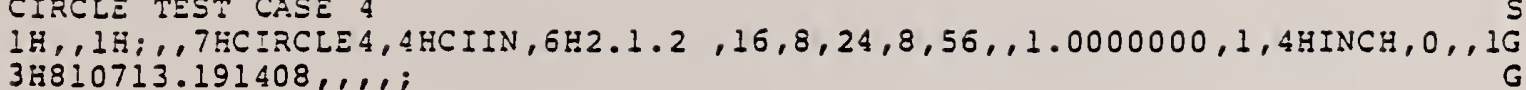

124

124

$i^{\prime \prime \prime \prime}$

100

100

100

100

$\begin{array}{lll}1 & 1 & 0 \\ 0 & 2 & 0 \\ 1 & 1 & 0 \\ 0 & 1 & 0 \\ 1 & 1 & 0 \\ 0 & 1 & 0\end{array}$

0

$\begin{array}{lllll}0 & 0 & 0 & 0 & \mathrm{D} \\ 0\end{array}$

$\begin{array}{lllll}0 & 0 & 0 & 0 & 0 \\ D\end{array}$

0

0

0

$\begin{array}{llllr}0 & 0 & 0 & 0 & D\end{array}$

$124,-.2499999, .4330126,-.8660254,0.0, .8080128,-.3995191$,

$-.4330126,0.0,-.5334937,-.8080127,-.2500000,0.0,0,0$;

$100,0.0,0.0,0.0,500.0000000,0.0,500.0000000,-.0003894,0,0$;

$100,0.0,0.0,0.0, .0050000,0.0, .0050000,-.3894144 \mathrm{E}-08,0,0$;

S

16

2D

$6 P$ 

3.3.2 Composite Entity

Case 1 (Figures 3.3.2-1 and 3.3.2-2) tests the following:

- Composite entities with the following subentities:

- 5 lines

- 2 circular arcs

- 1 cubic spline 


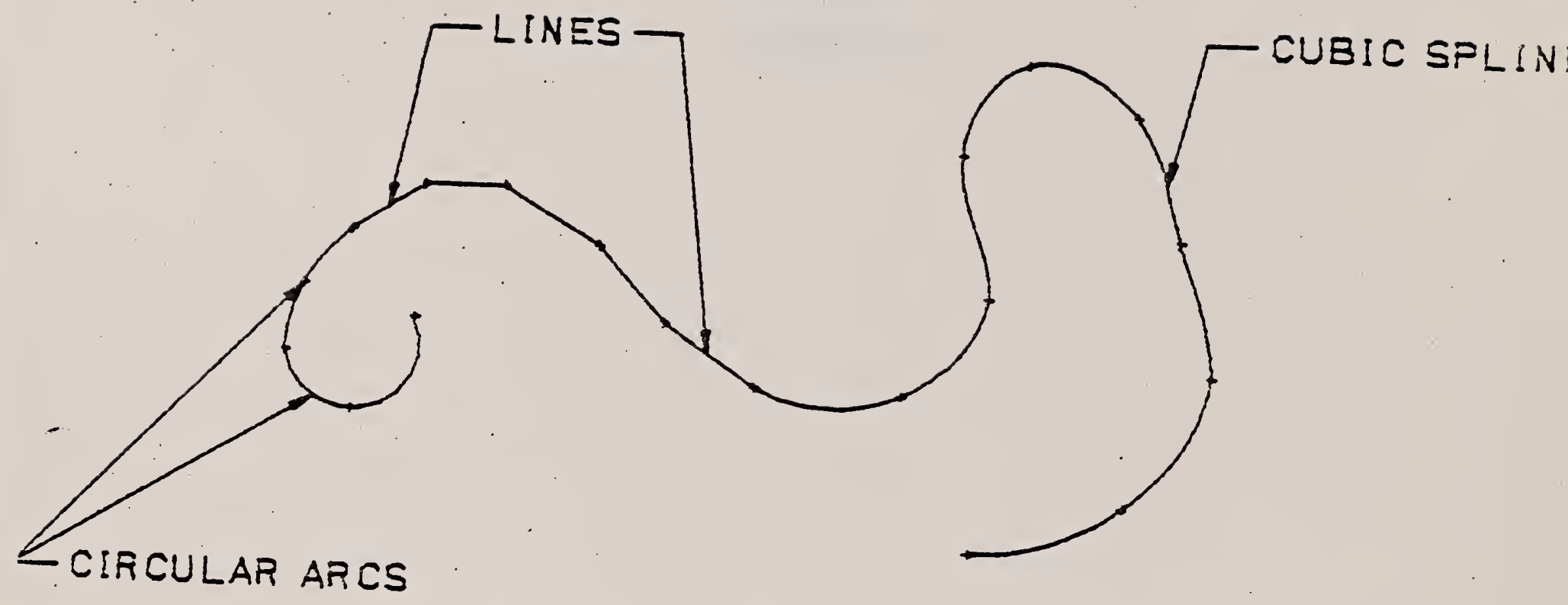

Figure 3+3.2-1 Composite Entity Case 1 (with annotation) Case 1: Rotation $\left(0^{\circ}, 0^{\circ}, 0^{\circ}\right)$ 


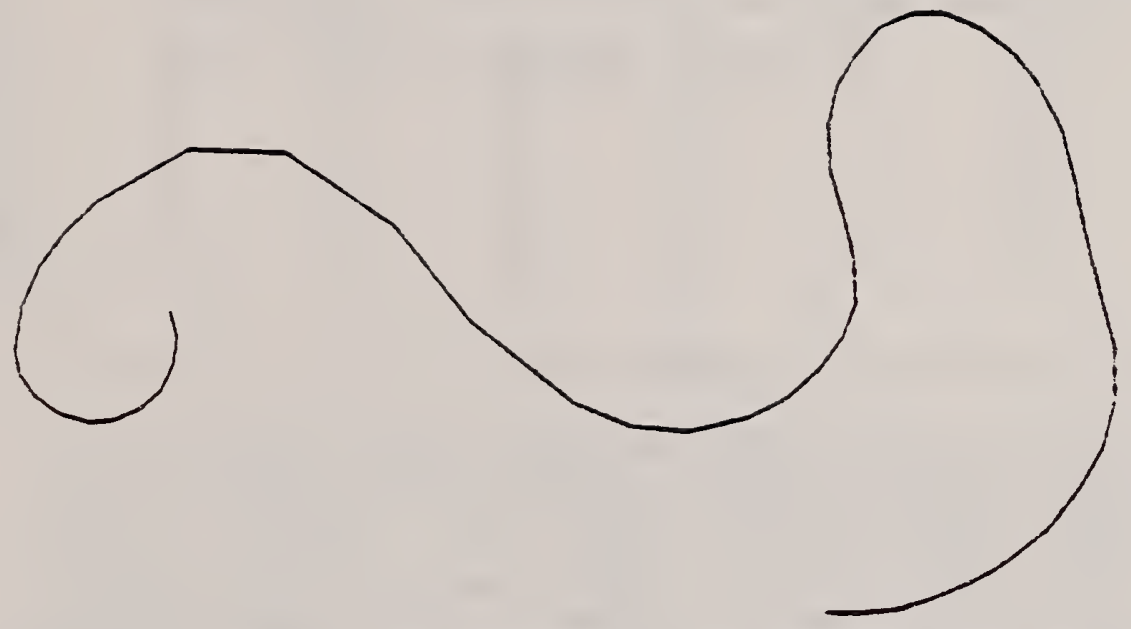

Figure 3.3.2-2 Composite Entity Case 1 (actual part)

$3.2-3$ 
COMPOSITE ENTITY TEST CASE ?

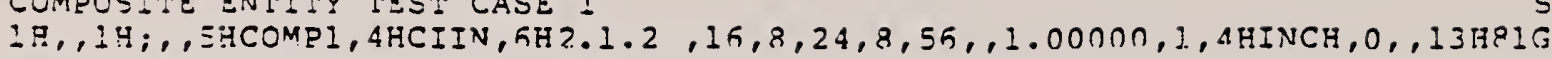

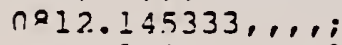

$\begin{array}{cr}102 & 1 \\ 102 & 0 \\ 124 & 3 \\ 124 & 0 \\ 112 & 5 \\ 112 & 0 \\ 110 & 20 \\ 110 & 0 \\ 110 & 21 \\ 110 & 0 \\ 110 & 22 \\ 110 & 0 \\ 110 & 2.3 \\ 110 & 0 \\ 110 & 24 \\ 110 & 0 \\ 110 & 25 \\ 100 & 0 \\ 100 & 25 \\ 110 & 0 \\ 102,9, & 5,\end{array}$

1
0
1
0
1
0
1
0
1
0
1
0
1
0
1
0
1
0
1
0

$\begin{array}{cc}1 & 0 \\ 2 & 0 \\ 1 & 0 \\ 2 & 0 \\ 1 & 0 \\ 15 & 0 \\ 1 & 0 \\ 1 & 0 \\ 1 & 0 \\ 1 & 0 \\ 1 & 0 \\ 1 & 0 \\ 1 & 0 \\ 1 & 0 \\ 1 & 0 \\ 1 & 0 \\ 1 & 0 \\ 1 & 0 \\ 1 & 0 \\ 1 & 0 \\ 11 & 13\end{array}$

0
0
0
0
0
0
0
0
0
0
$n$
0
0
0
0
0
0
0
0
0
15

0
0
0
0
3
0
0
0
0
0
0
0
0
0
0
0
3
0
3
0
17.

$i=4, i .00000,0.0,0.0,0.0,0.0,1.00000,0.0,0.0,0.0,0.0,1.00000,0.0$, 0,6 ;

$112,3,0,3,10,0.0,1.02455,2.05777,2.9794 \Delta, 3.85073,4.61954$, $5.35851,6.33129,7.18453,8.12094,8.12094,5.57500, .90989, .00585$, -. $05 \overline{5} \div, 1.51500,-.01453, .35101,-.01727,0.0,0.0,0.0,0.0,5.54400$, $.82834,-.17328,-.12053,1.95200, .55223, .20881,-.09500, n, 0, n .0$, $0.0,0.0,7.0720 n, .05555,-.57473, .28270,2.84 n n 0, .05545, .00437$, $.00315,0.0,0.0,0.0,0.0,5.85500,-.28449, .20579,-.321 .35,3.73500$, $.98153, .01307,-.07367,0.0,0.0,0.0,0.0,6.55200,-.55755,-.53423$, $.40704,4.55200, .93581,-.17926,-.46045,0.0,0.0,0.0,0.0,5.85500$, $-.91054, .30531, .24335,4.82000,-.25580,-1.2 .4210, .52433,0.0,0.0$, $0.0,0.0,5.44800,-.05 n 91, .8 \Delta 473,-.58673,4.254 n n,-1.05979, .34179$, $-.04043,0.0,0,0,0.0,0.0,5.5480 n,-.08313,-.85756, .25824,3.313 n n$, $-.93425,-.0 n 7.47, .21733,0.0,0.0,0.0,0.0,5.112 n 0,-.97778,-.12085$, $.16818,2.54800,-.45371, .55390,-.03339,0.0,0.0,0.0,0.0,4.1750 n$,

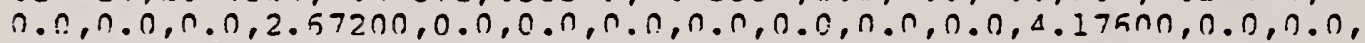
$n, 0,2.57200,0,0, n, 0,0,0,0,0,0, n, 0, n, 0,0,0,0$;

$=11,4.17500,7.67200,0.0,3.59200,3.0800 n, 0.0, n, 0$;

$110,3.59200,3.08000,0.0,3.15000,3.57500,0.0,0,0$;

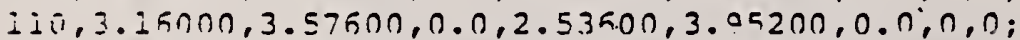

$110,2.53500,3.95200,0.0,2.02 .400,3.94400,0.0,0,0$;

$110,2.07 .1 n 0,3.94400,0.0,1.56 n n n, 3.632 n n, 0 . n, n, n$;

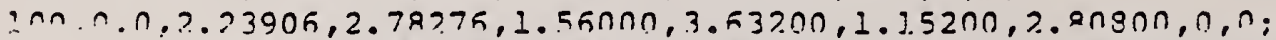

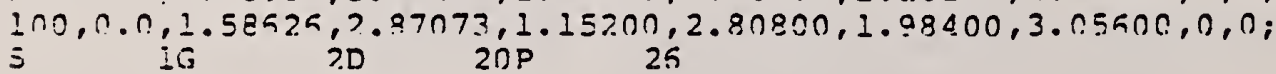

D

D on 


\subsubsection{Conic}

- Case 1 (Figures 3.3.3-1 and 3.3.3-2) tests the following:

- Closed and partial ellipses (FORM 1)

- Ellipses with fonts (solid, dashed, phantom, centerline)

- Symmetric and partial parabolas (FORM 3)

- Parabolas with fonts (solid, dashed, phantom, centerline)

- Symmetric and partial hyperbolas (FORM 2)

- Hyperbolas with fonts (solid, dashed, phantom, centerline)

- Case 2

- Identical to Case 1 except non-model space with rotation $\left(33^{\circ}, 77^{\circ}, 99^{\circ}\right)$ is used per section 3.0

NOTE: The start and terminate point of the conic may not exactly lie on the conic curve but may differ by $10^{-4}$ 
ELLPPSE
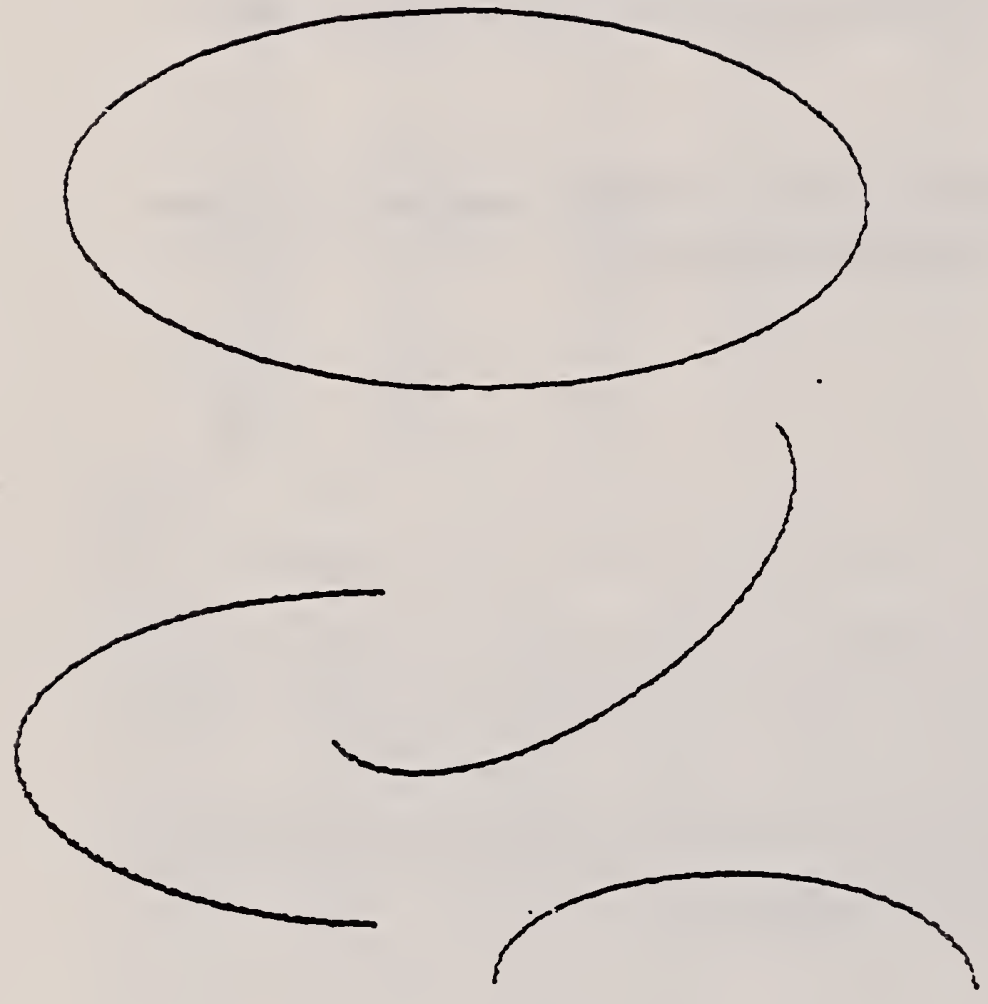

HYPERBOLA
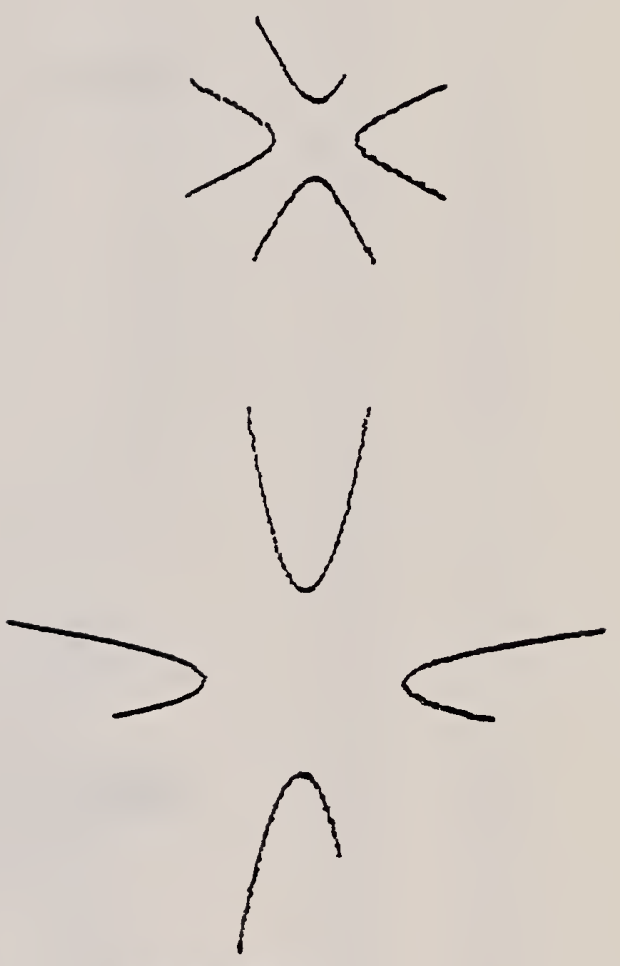

parabola

Figure 3.3.3-1 Conic Cases 1 and 2 (with annotation)

Case 1: Rotation $\left(0^{\circ}, 0^{\circ}, 0^{\circ}\right)$

Case 2: Rotation $\left(33^{\circ}, 77^{\circ}, 99^{\circ}\right)$ 

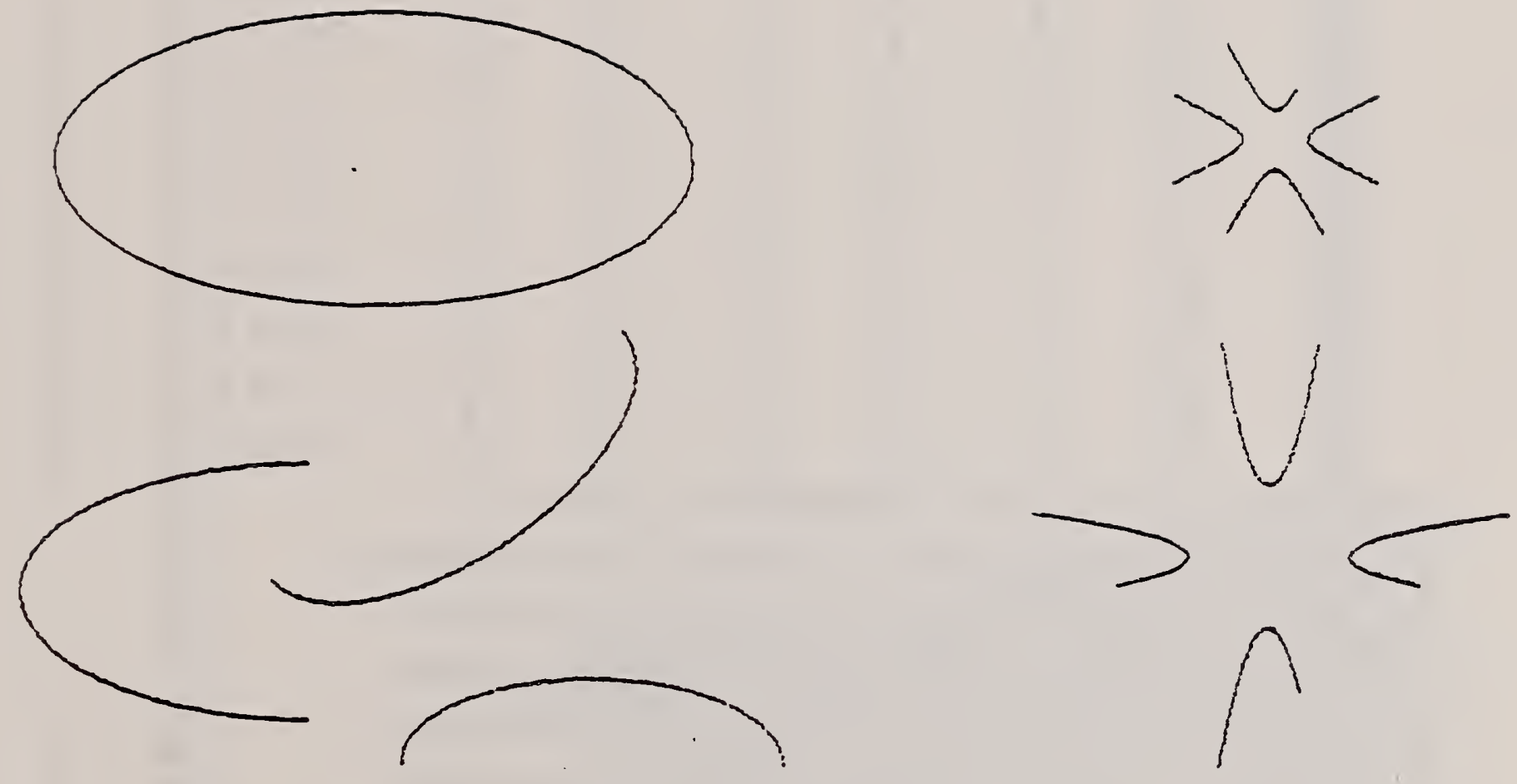

Figure 3.3.3-2 Conic Cases 1 and 2 (actual part) 
CONIC TEST CASE 1

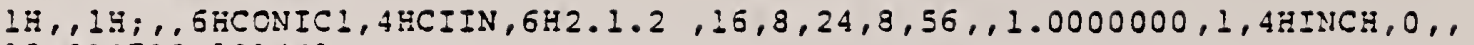

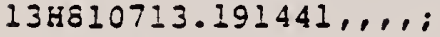

$\begin{array}{ccccccc}10713.191441 \ldots, \ldots & & & & 0 & 0 \\ 124 & 1 & 1 & 1 & 0 & 0 & 0 \\ 124 & 0 & 0 & 2 & 0 & 0 & 1 \\ 104 & 3 & 1 & 1 & 0 & 0 & 0 \\ 104 & 0 & 0 & 2 & 1 & 0 & 1 \\ 104 & 5 & 1 & 4 & 0 & 0 \\ 104 & 0 & 0 & 2 & 1 & 0 & 0 \\ 104 & 7 & 1 & 3 & 0 & 0 & 1 \\ 104 & 0 & 0 & 2 & 1 & 0 & 0 \\ 104 & 9 & 1 & 2 & 0 & 0 & 1 \\ 104 & 0 & 0 & 2 & 1 & 0 & 0 \\ 104 & 11 & 1 & 4 & 0 & 0 & 1 \\ 104 & 0 & 0 & 2 & 2 & 0 & 0 \\ 104 & 13 & 1 & 1 & 0 & 0 & 1 \\ 104 & 0 & 0 & 2 & 2 & 0 & 0 \\ 104 & 15 & 1 & 2 & 0 & 0 & 1 \\ 104 & 0 & 0 & 2 & 2 & 0 & 0 \\ 104 & 17 & 1 & 3 & 0 & 0 & 1 \\ 104 & 0 & 0 & 3 & 2 & 0 & 0 \\ 104 & 20 & 1 & 3 & 0 & 0 & 1 \\ 104 & 0 & 0 & 2 & 3 & 0 & 0 \\ 104 & 22 & 1 & 2 & 0 & 0 & 1 \\ 104 & 0 & 0 & 3 & 3 & 0 & 0 \\ 104 & 25 & 1 & 1 & 0 & 0 & 1 \\ 104 & 0 & 0 & 3 & 3 & 0 & 0 \\ 104 & 23 & 1 & 4 & 0 & 0 & 1 \\ 104 & 0 & 0 & 3 & 3 & 0 & 0\end{array}$

$124,1.0000000,0.0,0.0,0.0,0.0,1.0090000,0.0,0=0,0.0,0.0$,

$1.0000000,0.0,0,0$;

$104, .0025000,0.0,00100000,0.0,0.0,-1.0000000,0.0,20.0000000,0.0$, $20.0000000,-.1907349 \varepsilon-04,0,0$;

$104, .0030864,0.0, .0123457, .0246914, .7407407,10.1604938,0.0$,

$-3.9999957,-21.0000019,-4.0000086,-39.0000000,0,0$;

$104, .0110752,-.0149328, .0144350,-.4104291, .6609945,6.7296818$, $0.0,-6.1815600,-29.1839848,15.6827803,-11.5925392,0,0$;

$104, .0059444,0.0, .0277778,-.1944444,2.3333333,49.3611111,0.0$, $26.0000000,-42.0000000,2.0000010,-41.9999924,0,0$;

$104, .2500000,0.0,-1.0000000,-28.2500000,3.8000000,793.4525001$, $0.0,62.8245392,-1.0999905,62.8245544,4.8999991,0,0$;

$104,-.9999999, .5960464 \Sigma-05, .2500000,112.9999854,-.9500336$,

$-3192.3470556,0.0,57.8370590,5.2392955,53.5000038,8.2245541,0,0$;

$104, .2500000,-.1192093 \mathrm{E}-05,-1.0000000,-28.2499977,3.8000673$,

$793.4523721,0.0,50.1754646,4.8999934,50.1754455,-1.0999961,0,0$;

$104,-.9999999,-.29802325-06, .2500000,112.9999871,-.9499830$,

$-3192.3471515,0.0,53.5000114,-4.4245372,59.5000000,-4.4245529,0$, 0 ;

$104,0.0,0.0,1.0000000,-.9200000,54.2000008,790.8060245,0.0$, $65.5478271,-28.1000004,71.0826111,-24.1000004,0,0$;

$104, .9999999,-.4768371 E-06, .5684342 E-13,-112.6000034,-.9199731$, $3149.3585719,0.0,59.3000069,-12.3173933,53.3000069,-12.3173914$,

$\begin{array}{lllll}0 & 0 & 0 & 0 & 0\end{array}$

$\begin{array}{lllll}0 & 0 & 0 & 0 & 0\end{array}$

$\begin{array}{lllll}0 & 0 & 0 & 0 & D\end{array}$

00000

$\begin{array}{lll}0 & 0 & 0 \\ 0 & 0 & 0\end{array}$

$0 \quad 00000$

$\begin{array}{llllll}0 & 0 & 0 & 0 & D\end{array}$

$\begin{array}{lll}0 & 0 D & 12\end{array}$

$\begin{array}{llllll}0 & 0 & 0 & 0 & D & 13\end{array}$

$\begin{array}{lllll}0 & 0 & 0 & 0 & 0\end{array}$

14

0000017

$\begin{array}{llll}0 & 0 & 0 & 18\end{array}$

$\begin{array}{llll}0 & 0 D & 20\end{array}$

$\begin{array}{llllll}0 & 0 & 0 & 0 & D & 21\end{array}$

$\begin{array}{llllll}0 & 0 & 0 & 0.0 & 23\end{array}$

$\begin{array}{llllll}0 & 0 & 0 & 0 & D & 25\end{array}$

OD 26

12

19

$3 P$

$3 P$

$5 P$
$5 P$

$7 P$

$7 P$

98

$9 P$

112

II?

$13 ?$

13 ?

159

15 P

17 ?

179

178

19 ?

IOP

$21 \mathrm{P}$

21? 


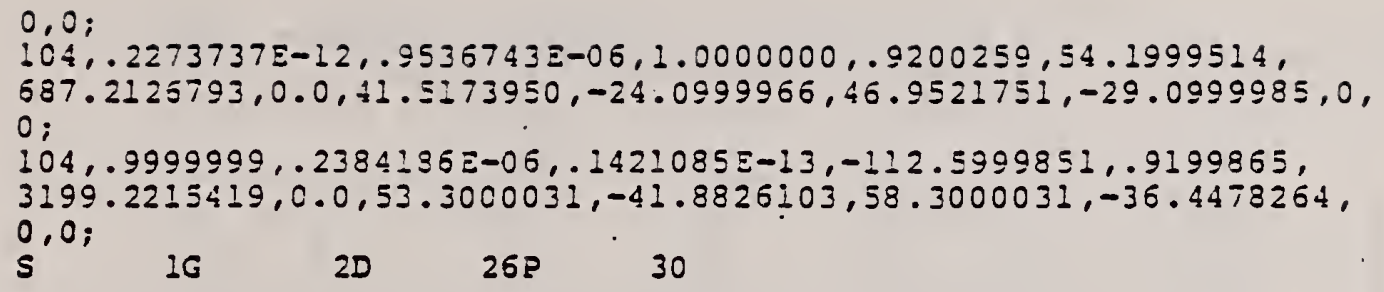

IG

2D

$26 P$

30

$\begin{array}{rr}21 P & 24 \\ 23 P & 25 \\ 23 P & 25 \\ 23 P & 27 \\ 25 P & 29 \\ 25 P & 29 \\ 25 P & 30 \\ T & 1\end{array}$



CONIC TEST CASE 2
$1 H, 1 H,, 6 H C O N I C 2,4 H C I N, 6 H 2.1 .2,16,8,24,8,56,1.0000000,1,4 \mathrm{HINCH}, 0,13 \mathrm{~S}$ H810713.191457, , , ;

$\begin{array}{lrr}124 & 1 & 1 \\ 124 & 0 & 0 \\ 104 & 3 & 1 \\ 104 & 0 & 0 \\ 104 & 5 & 1 \\ 104 & 0 & 0 \\ 104 & 7 & 1 \\ 104 & 0 & 0 \\ 104 & 9 & 1 \\ 104 & 0 & 0 \\ 104 & 11 & 1 \\ 104 & 0 & 0 \\ 104 & 13 & 1 \\ 104 & 0 & 0 \\ 104 & 15 & 1 \\ 104 & 0 & 0 \\ 104 & 17 & 1 \\ 104 & 0 & 0 \\ 104 & 19 & 1 \\ 104 & 0 & 0 \\ 104 & 22 & 1 \\ 104 & 0 & 0 \\ 104 & 24 & 1 \\ 104 & 0 & 0 \\ 104 & 27 & 1 \\ 104 & 0 & 0 \\ 104 & 30 & 1 \\ 104 & 0 & 0\end{array}$

$\begin{array}{ll}1 & 1 \\ 0 & 2 \\ 1 & 1 \\ 0 & 2 \\ 1 & 4 \\ 0 & 2 \\ 1 & 1 \\ 0 & 2 \\ 1 & 3 \\ 0 & 2 \\ 1 & 2 \\ 0 & 2 \\ 1 & 4 \\ 0 & 2 \\ 1 & 1 \\ 0 & 2 \\ 1 & 2 \\ 0 & 2 \\ 1 & 3 \\ 0 & 3 \\ 1 & 3 \\ 0 & 2 \\ 1 & 2 \\ 0 & 3 \\ 1 & 1 \\ 0 & 3 \\ 1 & 4 \\ 0 & 3\end{array}$

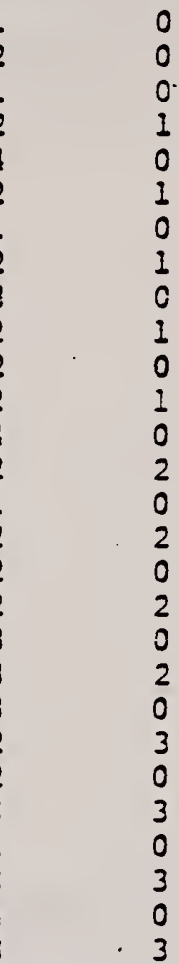

$\begin{array}{ll}0 & 0 \\ 0 & 0 \\ 0 & 0 \\ 1 & 0 \\ 0 & 0 \\ 1 & 0 \\ 0 & 0 \\ 1 & 0 \\ 0 & 0 \\ 1 & 0 \\ 0 & 0 \\ 1 & 0 \\ 0 & 0 \\ 2 & 0 \\ 0 & 0 \\ 2 & 0 \\ 0 & 0 \\ 2 & 0 \\ 0 & 0 \\ 2 & 0 \\ 0 & 0 \\ 3 & 0 \\ 0 & 0 \\ 3 & 0 \\ 0 & 0 \\ 3 & 0 \\ 0 & 0 \\ 3 & 0\end{array}$

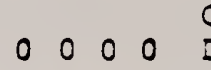

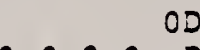

$\begin{array}{lllll}0 & 0 & 0 & 0 & D\end{array}$

$\begin{array}{llllll}0 & 0 & 0 & 0 & 0 & 0\end{array}$

$\begin{array}{llllll}0 & 0 & 0 & 0 & D\end{array}$

$\begin{array}{llllll}0 & 0 & 0 & 0 & 0 & 0\end{array}$

$\begin{array}{lllllllll}0 & 0 & 0 & 0 & 0 & 0 & 0 & 0 & 0\end{array}$

$\begin{array}{llllll}0 & 0 & 0 & 0 & 0\end{array}$

$\begin{array}{llllllll}0 & 0 & 0 & 0 & D & 0\end{array}$

$\begin{array}{lllllllll}0 & 0 & 0 & 0 & 0 & 0\end{array}$

$\begin{array}{lllll}0 & 0 & 0 & 0 & 0 \\ 0 & 0 & 0 & 0 & 0\end{array}$

00000

$\begin{array}{llllllllll}0 & 0 & 0 & 0 & 0\end{array}$

$\begin{array}{llllll}0 & 0 & 0 & 0 & D\end{array}$

$\begin{array}{llllll}0 & 0 & 0 & 0 & D\end{array}$

$\begin{array}{llllll}0 & 0 & 0 & 0 & D\end{array}$

0000 OD

$\begin{array}{lllll}0 & 0 & 0 & 0 & D\end{array}$

$O D$

$124,-.0351901, .2221817,-.9743701,0.0,-.9113618, .3929496$,

$.1225172,0.0, .4100994, .8923150, .1886600,0.0,0,0$;

$104, .0025000,0.0, .0100000,0.0,0.0,-1.0000000,0.0,20.0000000,0.0$, $20.0000000,-.1907349 E-04,0,0$;

$104, .0030854,0.0, .0123457,0.0,0.0,-1.0000000,0.0, .4291534 \mathrm{E}=05$, $8.9999990,-.8583069 \Xi-05,-9.0000000,0,0$;

$104, .0039063,0.0, .0156250,0.0,0.0,-1.0000000,0.0, .7629395 \mathrm{E}-05$, $-8.0000000, .1525879 \varepsilon-04,7.9999995,0,0$;

$104, .0051020,0.0, .0204082,0.0,0.0,-1.0000000,0.0,-13.9999990$, $.8344650 \Sigma-05,14.0000000,-.6675720 \varepsilon-05,0,0$;

$104, .0069444,0.0, .0277778,0.0,0.0,-1.0000000,0.0,12.0000000,0.0$, $-11.9999990, .7152557 \mathrm{E}-05,0,0$;

$104, .2500000,0.0,-1.0000000,-28.2500000,3.8000000,793.4525001$, $0.0,62.8245392,-1.0999905,52.8245544,4.8999991,0,0$

$104,-.9999999, .5960464 \mathrm{E}-06, .2500000,112.9999854,-.9500336$,

$-3192.3470556,0.0,59.4999924,8.2245359,53.5000038,8.2245541,0,0$;

$104, .2500000,-.1192093 \Xi-05,-1.0000000,-28.2499977,3.8000673$,

$793.4523721,0.0,50.1754646,4.8999934,50.1754456,-1.0999961,0.0$;

$104,-.9999999,-.29802325-06, .2500000,112.9999871,-.9499830$,

$-3192.3471516,0.0,53.5000114,-4.4245372,59.5000000,-4.4245529,0$, 0 ; 


$$
\begin{aligned}
& 104,0.0,0.0,1.0000000,-.9200000,54.2000008,765.0460240,0.0, \\
& 27 . \leqslant 4733: 0,-29.1000004,43.0826111,-24.1000004,0,0 \text {; } \\
& 104, .9995999,-.4768371 \mathrm{E}-06, .56843425-13,-56.0000063,-.9199865 \text {, } \\
& 780.5582742,0.0,31.3000031,-12.3173933,25.3000031,-12.3173914,0 \text {, } \\
& 0 \text {; } \\
& 104, .2273737 \Xi-12, .9536743 \Xi-06,1.0000000, .9200259,54.1999781 \text {, } \\
& 712.9734052,0.0,13.5173941,-24.0999966,18.9521751,-29.0999985,0 \text {, } \\
& 0 \text {; } \\
& 104, .9329999, .2384186 \mathrm{E}-06, .1421085 \mathrm{E}-13,-56.5999880, .9199932 \text {, } \\
& 6=0.42=7567,0.0,25.3000031,-41.8826103,30.3000011,-36.4478264,0 \text {, } \\
& \text { ก. } \\
& \text { S }
\end{aligned}
$$

$\begin{array}{rr}210 & 22 \\ 21 P & 23 \\ 23 P & 26 \\ 239 & 25 \\ 23 P & 26 \\ 250 & 27 \\ 259 & 28 \\ 25 P & 29 \\ 279 & 30 \\ 279 & 31 \\ 27 P & 32 \\ 7 & 1\end{array}$

$3.3-7$ 



\subsubsection{Copious Data Entity}

- Case 1 tests the following forms of the copious data entity:

- Level 1 (Figure 3.3.4-1) contains 2-D linear strings (FORM 11) in model space.

- Level 2 (Figure 3.3.4-2) contains 3-D linear strings (FORM 12) in a model space.

- Level 3 (Figure 3.3.4-3) contains a 3-D linear string (FORM 13) with associated normal vectors. Non-model space rotation $\left(90^{\circ}\right.$, $111.80^{\circ}, 125.94^{\circ}$ ) is used per section 3.0. 


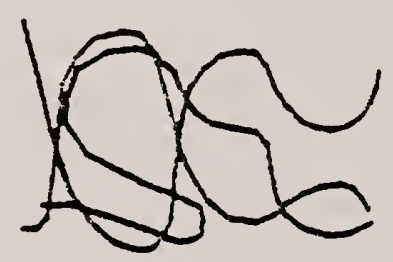

Figire 3.3.4-1 Linear String Form 11 (actual part) Case 1: Rotation $\left(0^{\circ}, 0^{\circ}, 0^{\circ}\right)$ 

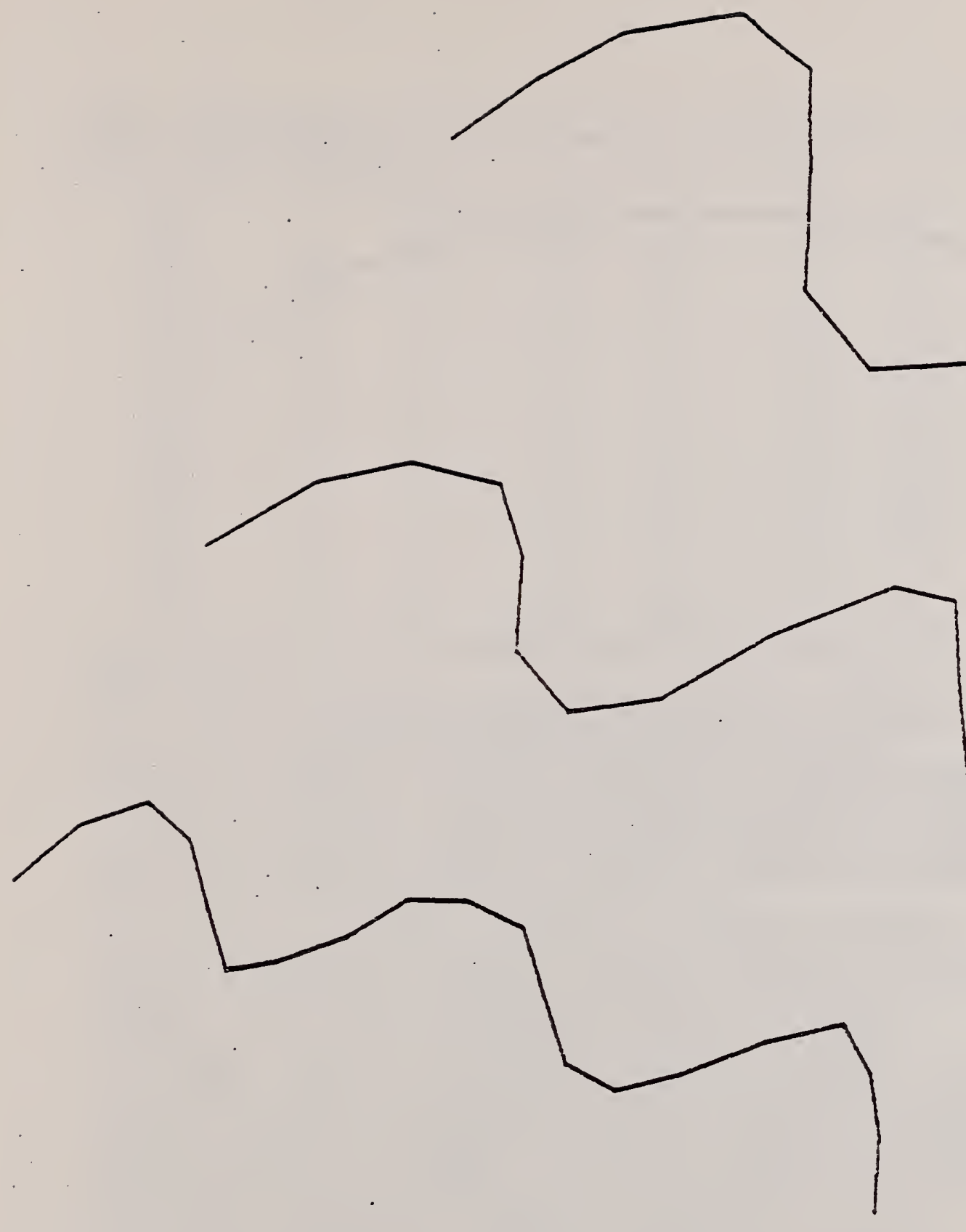

Figure 3.3.4-2 Linear String Form 12 (actual part) Case 1: Rotation $\left(0^{\circ}, 0^{\circ}, 0^{\circ}\right)$

IGES Test Def.

$3.4-3$ 


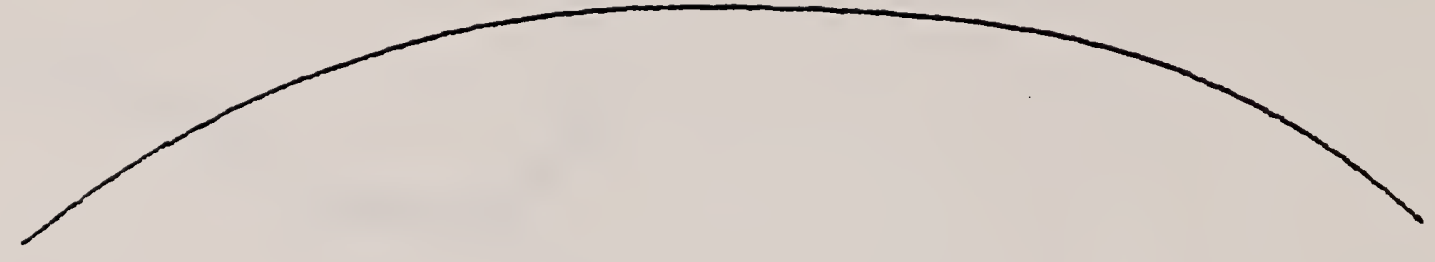

Figure 3.3.4-3 Linear String Form 13 (actual part) Rotation: $\left(90^{\circ}, 111.80^{\circ}, 125.94^{\circ}\right)$ 
IENEAR SIRING CASE I

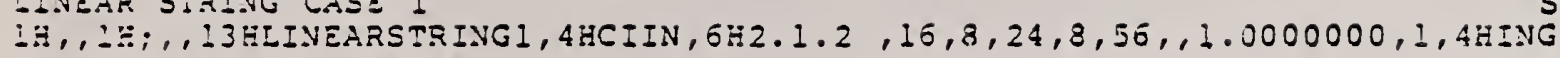
CH, $0,135810714.085946, \ldots$, ;

$\begin{array}{rrrrrrr}124 & 1 & 1 & 1 & 0 & 0 & 0 \\ 124 & 0 & 0 & 2 & 0 & 0 & 0 \\ 106 & 3 & 1 & 1 & 1 & 0 & 1 \\ 106 & 0 & 0 & 9 & 11 & 0 & 0 \\ 106 & 12 & 1 & 1 & 1 & 0 & 1 \\ 106 & 0 & 0 & 12 & 11 & 0 & 0 \\ 106 & 24 & 1 & 1 & 1 & 0 & 1 \\ 106 & 0 & 0 & 12 & 11 & 0 & 0 \\ 106 & 36 & 1 & 1 & 2 & 0 & 1 \\ 105 & 0 & 0 & 16 & 12 & 0 & 0 \\ 106 & 52 & 1 & 1 & 2 & 0 & 1 \\ 106 & 0 & 0 & 13 & 12 & 0 & 0 \\ 106 & 65 & 1 & 1 & 2 & 0 & 1 \\ 106 & 0 & 0 & 11 & 12 & 0 & 0 \\ 124 & 76 & 1 & 1 & 0 & 0 & 0 \\ 124 & 0 & 0 & 2 & 0 & 0 & 0 \\ 106 & 78 & 1 & 1 & 3 & 0 & 15 \\ 106 & -0 & 0 & 224 & 13 & 0 & 0\end{array}$

$124, ? .0000000,0.0,0.0,0.0,0.0,1.0000000,0.0,0.0,0.0,0.0$, $1.0000000,0.0,0,0$;

$106,1,24,0.0,-17.0832386,5.6932182,-16.5788116,5.7789435$,

-i5.7591171,5.1168823,-14.6556816,4.8016148,-13.3000326,

$4.8961949,-11.9443836,5.4321494,-10.9040022,5.0942106$,

$-9.7059860,7.1030655,-8.1611767,7.7651257,-0.1434669,7.5444398$,

$-4.5671306,6.2203174,-3.4952223,4.7070346,-2.5494206,2.8784850$,

$-1.2883517,-1.4722028,-.1849164,-5.2869363, .9500450,-6.7056384$,

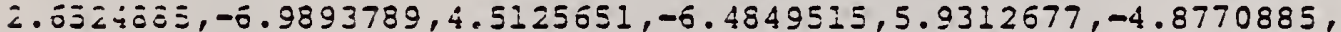
$6.9401226,-2.7963250,7.3814969,2.4371109,7.9805045,7.5444398$, $8.8001395,8.3326082,9.8721075,8.3956614,0,0$;

$106,1,32,0.0,-17.8714066,-4.1204472,-17.5876675,-2.8278518$,

$-17.0832386,-1.6298363,-16.3896523,-.5264010,-15.4753771$,

$.1356601,-14.0566740, .5455075,-12.8536588, .3878739,-11.6921701$,

$-.0850270,-10.6517878,-1.1254088,-9.9897270,-2.0712104$,

$-9.4852991,-3.1746457,-5.9493456,-4.3725611,-8.1927042$,

$-5.3815165,-6.7424746,-5.6022034,-5.2607 i 88,-5.1608291$,

$-4.0311760,-4.1204472,-3.0538480,-2.7017448,-2.2656801, .4194005$,

$-1.8558327,4.2341342,-1.5720922,7.4498596,-.9730845,8.9946690$,

$-.0903363,0.8450900,1.3283663,9.9719973,2.7785954,9.4990959$,

$4.15577: 0,8.3641348,5.2592065,7.1030655,6.1204281,5.6529363$,

$5.071 \leq 406,3.4459660,7.6967640,1.0814613,8.3508257,-1.5657830$,

$9.0208864,-4.4357147,9.9351615,-7.7144938,0,0$;

1.5:-1,34,0.0,-27.2408733,7.7536001,-15.6014833,8.5532951,

$-13.3044605,8.7739820,-11.9759102,8.3010807,-10.5887346$,

$7.1551191,-9.8951464,5.9581039,-9.4852991,3.7512331,-9.1069784$,

i. $8055764,-2.2557573, .7661945,-6.2695737, .4194005,-4.5671306$,

$.104-334,-3.3091154,-.7786148,-2.4863672,-2.1342540,-1.7297258$,

- Z . 7-35:35, -.2794966, $-5.0977759,1.3283663,-5.7598372,3.5352368$,

$-5.44 \leq 5 \leq ? 5,5.5475272,-4.0889206,6.4356952,-1.9135768,6.6243555$,

$.2302403,5.1746254,2.4636375,2.9362290,3.6656529, .8369921$,

$\begin{array}{lllll}0 & 0 & 0 & 0 & 0\end{array}$

$\begin{array}{llllll}0 & 0 & 0 & 0 & 0\end{array}$

$51 \quad O D$

$\begin{array}{llllllll}0 & 0 & 0 & 0 & D\end{array}$

52

$\begin{array}{lllll}0 & 0 & 0 & 0 & D\end{array}$

\begin{tabular}{llllll}
\hline & 0 & 0 & 0 & 0 & 0
\end{tabular}

$54 \quad O D$

$\begin{array}{lllll}0 & 0 & 0 & 0 & D\end{array}$

55

$\begin{array}{lllll}0 & 0 & 0 & 0 & 0\end{array}$

s6

0

OD

IP

19

$3 p$

$3 P$

39

3 ?

39

$3 P$

$3 P$

$3 P$

$3 P$

$5 P$

$5 ?$

$5 P$

25

$5 P$

$\sum p$

S?

25

$5 P$

Is

$5 P$

SP

79

78

$7 ?$

75

7

$7 p$

ip

75 
$4.5809278,-1.8243060,5.6843634,-3.8420162,6.5932182,-4.0942302$, $7.733500 \div,-3.9681232,8.6794014,-3.2114818,9.1207762,-2.1395731$, $9.3095365,-.9415578,8.9631424, .6032516,0.3641348,3.5667634$, $7.3552794,6.4987488,6.4725313,8.3588257,6.5986381,0,0 ;$ $106,2,20,-166436.2343750,-79594.1875000,-155032.1718750$, - $150317.2656250,-65993.6484375,-146587.0468750,-133174.8750000$, $-59756.8515625,-128580.8515625,-122428.9062500,-68533.1171875$, $-104607.4765625,-117311.7656250,-85383.2890625,-80520.5859375$, $-112450.4921875,-100111.6540625,-58917.3320313,-99913.5234375$, $-976 \div 3.1093750,-43655.9414063,-32259.4140625,-91044.4687500$, $-25287.9003906,-67163.8750000,-31649.5937500,-13334.4619141$, $-53091.7617188,-81677.5156250,6594.4301758,-38507.9335938$, $-37484.1640625,33025.7148438,-32879.0859375,-103972.4921875$, $57474.4375000,-26738.5292969,-121122.4218750,83308.4218750$, $-14201.5556641,-127352.9765625,107268.9375000,1917.4097900$, $-122863.2812500,125574.8906250,22385.9375000,-114018.6875000$, $145677.1718750,41575.1835938,-108893.1171875,167689.2812500$, $48995.0273438,-121300.5937500,190590.0000000,51809.4492188$, $-138499.7500000,211769.3593750,51553.5898438,-157358.2031250$, $230265.9687500,0,0$;

$106,2,16,-120893.7656250,3680.5187988,-174650.0937500$,

$-93261.2578125,20149.3750000,-152040.6875000,-69466.5859375$, $25461.1933594,-123701.7734375,-47462.9218750,20551.6074219$, $-87574.3045875,-41322.3593750,2889.9716797,-61328.6093750$, $-42089.9296875,-19912.3046875,-39611.8398438,-28735.3847656$, $-34555.08 @ 8438,-5153.5888672,-5758.2924805,-30553.5976563$, $22410 .: 406250,20850.7910156,-14296.7001953,43784.1679688$, $51041.8671875,-1647.5095215,73831.6015625,66137.4062500$, $-4533.7514648,98066.1540625,68184.2656250,-27136.9277344$, $123564.0234375,71510.3984375,-54208.5039063,155339.4687500$, $79697.8125000,-65817.3750000,178527.0781250,102980.7656250$, $-57797.1171875,203433.8906250,129529.8515625,-35911.3750000$, $219179.0781250,0,0$

$106,2,14,-62302.6093750,104725.3046875,-192834.4687500$, $-41578.2226563,120147.2265625,-178947.6875000,-20086.2753906$, $131762.3281250,-160168.5093750,9081.3789063,137547.2968750$, $-124704.2812500,26223.7753906,124339.0000000,-87252.9765625$, $27247.2011719,98965.2968750,-60431.9335938,26735.4882813$, $69435.8046875,-31626.1191406,43366.1679688,50748.5429688$, $10580.4794922,75348.2421875,53662.3203125,52896.1796875$, $106818.6093750,71565.6640625,79498.6406250,133683.5468750$, $83553.9218750,105403.1484375,161060.2031250,82799.4687500$, $144974.0312500,161571.9218750,46829.6679688,181667.5000000$, : $\$ 24, .2179680,-.3007008,-.9284767,0.0,-.5449199, .7517521$, -.37j3907,0.0,.8096618,.5868967,-.8970038E-14,0.0,0,0; $106,3,195,168.1533374,108.7943474,0.0,168.1664308,-98.3623479$, $15155.7416570,168.2556492,108.8837242,0.0,162.2758157$, $-08.9032742,15065.4584928,168.6856710,109.2223890,0.0$, $168.6971194,-100.9649623,14731.9961143,169.1074731,109.5593993$,

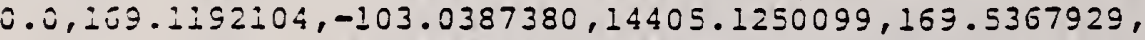

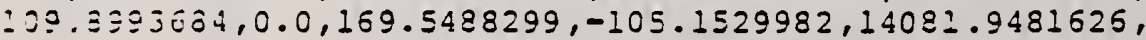

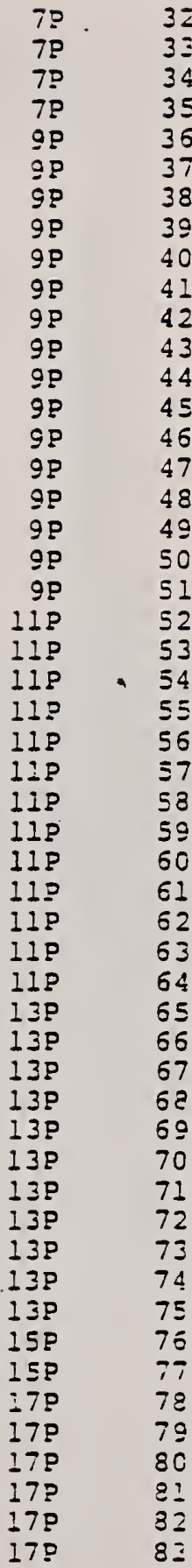


LE9.9679577,110.2377732,0.0,169.9803011, -107.2799994 , $13757.2953415,170.4047717,110.5775887,0.0,170.4174306$, $-109.4385798,13458.4059555,170.8435136,110.9159089,0.0$, $170.8564942,-111.6108253,13158.4482103,171.2844427,111.2529576$, $0.0,171.2977512,-113.7978491,12867.1984279,171.7274050$, $111.5886397,0.0,171.7410472,-115.9991199,12584.7576772$, :72.1501739,111.9063461,0.0,172.1641382,-118.1039967, $12324.5059592,172.5753759,112.2233022,0.0,172.5896676$, $-120.2249470,12071.7289575,173.3822629,112.8178499,0.0,$. $173.3971843,-124.2609107,11615.9766696,173.7569628,113.0909364$,

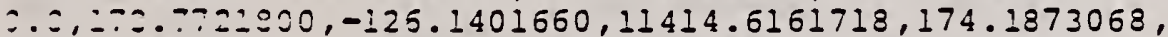
$113.4015909,0.0,174.2031292,-129.7517877,11004.8746185$, $174.2195015,113.4247120,0.0,174.2354764,-130.0623721$, $10970.4157810,174.2296242,113.4318821,0.0,174.2455155$, $-130.1588534,10959.7583124,174.4031208,113.5555821,0.0$, $174.4192959,-131.8358395,10777.9647968,174.7610898,113.8083322$, $0.0,174.7778562,-135.3372938,10418.8216886,175.1905922$, $114.1072438,0.0,175.2080760,-139.6133442,10015.4214943$, $175.6521569,114.4232694,0.0,175.6704183,-144.3018468$, Q. $\$ 3.7214350,176.1323519,114.7464328,0.0,176.1514263$, $-149.2848761,9228.5275207,176.6254366,115.0724155,0.0$, $176.5453472,-154.5166159,8865.0531473,177.1285144,115.3990012$, $0.0,177.1492771,-159.9780779,8524.8132709,177.6399517$, $115.7249109,0.0,177.6615771,-165.6624023,3207.6484915$, $178.1587543,116.0493420,0.0,178.1812497,-171.5691331$, $7312.5787519,178.6242871,115.3717589,0.0,178.7076569$, $-177.7016548,7638.2151628,179.2161327,115.6917375,0.0$, $179.2403797,-184.0659421,7382.9778762,179.7540156,117.0091580$, $0.0,179.7791416,-190.6699133,7145: 2182762,180.2977595$, 117 ₹2?5712,0.0,180.3237560,-197.5230887,6923.2890438, $180.8472612,117.6351789,0.0,180.8741500,-204.6364190$ 。 E:15. $5839773,181.4024758,117.9435706,0.0,181.4302495$, $-212.0222136,6520.5604579,181.9634068,118.2487654,0.0$, $181.9920698,-219.6941340,6336.7503077,182.5301013,118.5507066$, $0.0,182.5596599,-227.6672339,6162.7659876,183.1026469$, $118.8493581,0.0,183.1331103,-235.9580344,5997.3019045$, $\div 82.6 \varepsilon 11708,1: 9.1447013,0.0,183.7125514,-244.5846301$, $5839.2347035,184.2558404,119.4367341,0.0,184.2981546$, $-253.5668237,5687.1218570,184.8568643,119.7254692,0.0$, $184.8901330,-262.9262893,5540.2015016,195.4544952,120.0109334$, $0.0,185.4887445,-272.6867658,5397.3913086,186.0590325$, $120.2931670,0.0,186.0942943,-282.8742838,5257.7895054$, $186.6708267,120.5722242,0.0,186.7071391,-293.5174299$, $=120.5747314,187.2902778,120.8481693,0.0,187.3276861$, $-304.64754 \div 7,4985.0100898,187.9178518,121.1210826,0.0$, $: 37.9564090,-316.2902487,4850.4419049,188.5519739,121.3901740$, 0.0, 3 $\varepsilon .5917369,-328.4696509,4716.7453146,189.1948579$, $: 2: .6552731,0.0,189.2358959,-341.2291759,4583.0290825$, Z: . $4575.396: 455,189.8857060,121.9348470,0.0,189.9231779$, $-355.4288930,4441.2409496,190.5525083,122.1966275,0.0$, $: 50.5964343,-359.6290627,4305.8470490,191.2310317,122.4560086$, 
$0.0,191.2765132,-384.5929631,4169.5205225,191.9223123$, $122.713: 548,0.0,191.9694621,-400.3867536,4032.1592012$, i $92.6275306,122.9682900,0.0,192.6764736,-417.0849059$, $3893.7586092,193.3480249,123.2215962,0.0,193.3988986$, $-434.7713151,3754.3944248,194.0853023,123.4733110,0.0$, $194.1382566,-453.5405326,3614.2381635,104.8397506,123.7232508$, $0.0,194.8949441,-473.4641251,3473: 7830855,195.6114815$, $123.971-459,0.0,195.6690785,-494.6087485,3333.6620716$, 196.2289195,124.1639741,0.0,196.2885187, -512.0910759 , $3224.4505337,196.8455565,124.3517953,0.0,196.9072211$, $-570.0579457 .3118 .1939252,197.7492308,124.6205264,0.0$, $197.8131919,-547.6540644,3011.2497571,198.4579530,124.8267860$, $0.0,198.5237586,-565.2390018,2929.0950487,199.0427703$, $124.9950007,0.0,199.1101096,-584.4307571,2862.5133828$, $199.5470941,125.1326787,0,0,199.6157020,-605.4684849$, $2808.1785794,200.0000572,125.2546599,0.0,200.0697200$, $-620.6542449,2762.9896793,200.4239939,125.3654002,0.0$, $200.4945559,-554.4429687,2723.8914526,200.8412329,125.4708175$, $0.0,200.9126139,-683.7083642,2687.2909330,201.2836190$, $125.5784772,0.0,201.3558624,-718.3942663,2647.6469743$, $201.8011875,125.6990019,0.0,201.8745909,-761.9552739$, $2594.1261970,202.3471741,125.8201658,0.0,202.4222857$, $-607.0881329,2519.1492796,202.8055459,125.9178151,0.0$, $202.8824083,-843.5855882,2445.5679691,203.3635391,126.0319454$, $0.0,203.4424699,-891.2365655,2358.3997663,203.9296040$, $126.1424782,0.0,204.0105158,-943.5489277,2273.0052638$, $204.5047800,125.2494598,0.0,204.5875305,-1001.2517222$, $2189.6249269,205.0901854,126.3529201,0.0,205.1745663$, $-1065.2343922,2108.4435789,205.6870302,126.4528719,0.0$, $205.7727565,-1136.5934843,2029.5972927,206.2966317,126.5493101$, $0.0,206.3833311,-1216.6966889,1953.1798494,206.9204331$, $125.6422096,0.0,207.0076358,-1307.2722179,1879.2488998$, $207.5600246,126.7315238,0.0,207.6471569,-1410.5360424$, $1807.8313969,208.2171695,126.8171819,0.0,208.3035510$, $-1529.3771299,1738.9286398,208.8938352,126.8990861,0.0$, $208.9786833,-1667.6340502,1672.5206576,209.5922313,126.9771073$, $0.0,209.6746737,-1830.5202468,1608.5701087,210.3148531$, $127.0510801,0.0,210.3939494,-2025.2995091,1547.0258166$, $211.0645305,127.1207958,0.0,211.1393028,-2262.4014499$, $1487.8262299,211.8442441,127.1859744,0.0,211.9137157$, $-2557.2523407,1430.9194320,212.6570793,127.2462576,0.0$, $212.7203177,-2933.6342586,1376.2564920,213.4875978,127.3000876$, c.0,213.5439164,-3417.9032162,1324.9418113,214.3366030, $:=7.34 .2372,0.0,214.3854767,-4062.7232432,1276.7826557$, $215.1677049,127.3862957,0.0,215.2091082,-4916.3943556$, $1233.40104: 7,216.0051614,127.4185676,0.0,216.0390058$, $-5138.6159839,1193.3454423,216.8550461,127.4444652,0.0$, $216.8813681,-8045.7613262,1155.8769487,217.7428508,127.4644810$, $0.0 .217 .7613849,-11565.2382906,1119.9206167,218.6719573$, $127.4780936,0.0,218.6826896,-20217.5160886,1085.4417658$, 2:3.696595三, $127.4847170,0.0,219.6495096,-75239.0465783$, 1352. 3\$12586,220.1592546,127.4851932,0.0,220.1602924, 
$212262.5523205,1036.1772655,220.6719896,127.4835718,0.0$, $220.6768567,45362.5220054,1020.7101283,221.2284685,127.4798258$, $0.0,221.2373837,24921.0298033,1004.7235754,221.7849891$, $127.4737637,0.0,221.7978095,17397.0797041,989.5209269$, $222.3758385,127.1649789,0.0,222.3926614,13308.2949348$, $974.1884621,222.9667175,127.4538526,0.0,222.9873981$, $10863.7895923,959.6371863,223.5970660,127.4394887,0.0$, $223.6217066,9149.5970178,944.9222953,224.2274300,127.4226381$, $0.0,224.2558752,7951.8239434,930.9890304,224.9028942$, $127.4019210,0.0,224.9352503,7013.8626398,916.8703483$, $225.5783584,127.3785522,0.0,225.6144606,6305.9061705$, $903.5359138,226.3057053,127.3505372,0.0,226.3456657$, $5715.6538177,389.9944745,227.0330353,127.3196820,0.0$, $227.0765802,5249.6096258,877.2424225,227.8204806,127.2832058$, $0.0,227.8679292,4844.7759393,864.2624729,228.6078900$, $127.2436731,0.0,228.6589600,4515.8152234,852.0799204$, $229.4655180,127.1972894,0.0,229.5203371,4221.5642524$, $839.6495048,230.3230892,127.1475987,0.0,230.3814659$, $3977.8591494,828.0278090,231.2634303,127.0894888,0.0$, $231.3255024,3754.9629202,816.1396158,232.2026913,127.0277794$, $0.0,232.2692573,3568.0050953,805.0753309,233.2425285$, $126.2556260,0.0,233.3117374,3393.9617380,793.7279116$, $234.2812593,125.3795290,0.0,234.3538987,3246.9172358$, $783.2241467,234.8587052,126.8355855,0.0,234.9334986$, $3159.9628145,777.9393647,235.4348102,126.7892475,0.0$, $235.5150121,2948.4124121,774.8133054,236.0087684,126.7396356$, $0.0,236.0943007,2765.4348370,771.5524579,236.8398942$, $126.6615642,0.0,236.9330756,2538.3545304,766.7620336$, $237.6670834,126.5767795,0.0,237.7678251,2346.5838777$, $762.0895218,238.4860587,126.4854586,0.0,238.5942515$, $2182.6595817,757.6736176,239.2927918,126.3882808,0.0$, $239.4082952,2041.2995131,753.5789294,239.4222339,126.3720110$, $0.0,239.5389080,2020.2330718,752.9381208,240.2046068$, $126.2696244,0.0,240.3283360,1901.0485052,749.2512489$. $240.9763373,126.1617209,0.0,241.1069849,1795.8149370$, $745.8385012,241.7376261,126.0484165,0.0,241.8750488$, $1702.2543293,742.6344356,242.4855419,125.9303370,0.0$, $242.6295617,1618.9104823,739.6212809,243.2210690,125.8075515$, $0.0,243.3715118,1544.1656501,736.7578949,243.9451270$, $125.6801109,0.0,244.2018241,1476.5967581,734.0649967$, $244.6583700,125.5480850,0.0,244.8211594,1415.4140054$, $731.5309585,245.3610170,125.4116031,0.0,245.5297413$, $1359.4147513,729.2152445,246.0528256,125.2709564,0.0$, $246.2271780,1309.2571107,727.4659823,246.7415957,125.1246129$, $0.0,246.9215875,1261.1038215,726.5633868,247.4179314$, $124.9744614,0.0,247.6034795,1215.4909801 .726 .3820147$, $248.0534710,124.8249121,0.0,248.2543013,1173.5635336$, $726.7638016,248.6833467,124.6753477,0.0,248.8791998$, $1135.1538520,727.5575847,249.2820407,124.5251571,0.0$, $249.4826757,1099.5371255,723.6496141,249.8631976,124.3738085$, $0.0,250.0683915,1066.4533803,729.9517469,249.9415521$, $124.3529757,0.0,250.1473540,1062.1125494,730.1301221$,

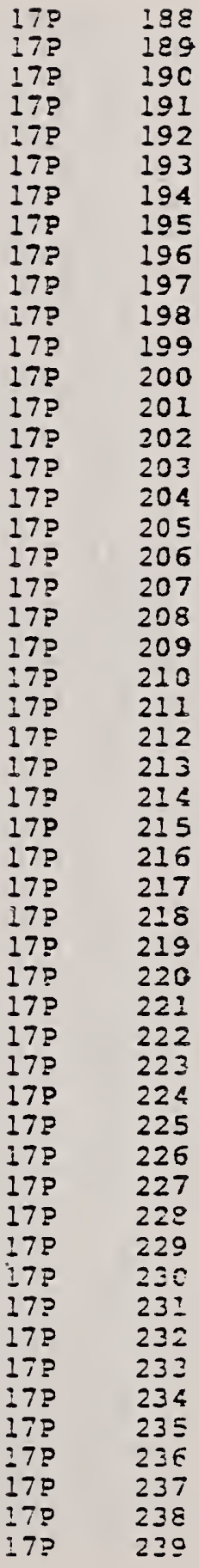


$250.4989696,124.2013695,0.0,250.7090959,1032.8924793$,

$729.8753 .426,251.0639183,124.0434611,0.0,251.2783611$,

$1004.4853333,729.7255299,251.6227420,123.8814334,0.0$,

$251.8413841,977.5029551,729.6955642,252.1709439,123.7172018,0.0$, $252.3936335,052.0500476,729.7785240,252.7088019,123.5508607,0.0$, $252.9353893,928.0020683,729.9656609,253: 2367447,123.3824493,0.0$, $253.4670841,905.2416538,730.2483067,253.7552228,123.2119883,0.0$, $253.9891726,883.6631002,730.6179404,254.2646800,123.0394878,0.0$, $254.5021028,863.1719211,731.0661705,254.7655511,122.8649486,0.0$, $255.0063141,843.6834283,731.5847472,255.2582611,122.6883626,0.0$, $255.5022359,825.1215000,732.1655542,255.7432243,122.5097132,0.0$, $255.9902875,807.4175252,732.8005999,256.2208443,122.3289760,0.0$, $256.4708771,790.5094977,733.4820135,256.6915137,122.1461193,0.0$, $256.9444023,774.3412425,734.2020246,257.1556140,121.9611037,0.0$, $257.4112496,758.8617410,734.9529656,257.6135161,121.7738834,0.0$, $257.8717949,744.0245532,735.7272542,258.0655804,121.5844054,0.0$, $258.3264036,729.7873128,736.5173867,258.5121569,121.3926105,0.0$, $258.7754309,716.1112897,737.3159242,258.9535361,121.1984330,0.0$, $259.2192222,702.9610048,738.1154874,259.3901988,121.0018008,0.0$, $259.6581138,690.3038937,738.9087493,259.8223173,120.8026355,0.0$, $260.0924329,678.1100120,739.6884259,260.2502552,120: 6008527,0.0$, $260.5224983,666.3517740,740.4472713,250.6743185,120.3563615,0.0$, $260.9486212,655.0037241,741.1780689,251.0948058,120.1890647,0.0$, $261.3711053,644.0423234,741.8736461,261.5120088,119.9788589,0.0$, $251.7902472,633.4457991,742.5268494,261.9262129,119.7656340,0.0$, $262.2063377,623.1939296,743.1305608,262.3375976,129.5492734,0.0$, $262.6196613,613.2679520,743.5776944,262.7467373,119.3296537,0.0$, $263.0304972,603.6504067,744.1612071,263.1536012,119.1066447,0.0$, $263.4391199,594.3250294,744.5740964,263.5585544,118.8801091,0.0$, $263.8457992,585.2766446,744.9094228,263.9618575,118.6499021,0.0$, $264.2508010,576.4910748,745.1603073,264.3637680,118.4158717,0.0$, $264.6543873,567.9550547,745.3199560,264.7645397,118.1778579,0.0$, $265.0568168,559.6561540,745.391 .6800,255.1644235,117.9356930,0.0$, $265.4583454,551.5827115,745.3389035,265.5636675,117.6892013,0.0$, $265.8592257,543.7237694,745.1851997,265.9625173,117.4381986,0.0$, $266.2597080,536.0690171,744.9143149,266.3612159,117.1824923,0.0$, $266.6600401,523.6087394,744.5201974,266.7600041,116.9218813,0.0$, $267.0604672,521.3337677,743.9970403,267.1591202,116.6561558,0.0$, $267.4612320,514.2354375,743.3393121,267.5588000,116.3850972,0.0$, $267.8625745,507.3055475,742.5418061,267.9592762,116.1084784,0.0$, $258.2647320,500.5363226,741.5996883,268.2344489,115.9154752,0.0$, $268.5410720,495.9849496,740.8611570,253.6372034,115.6282994,0.0$, $268.9449562,499.1576074,741.7972167,269.0120147,115.3565583,0.0$, $269.3217087,483.4292899,739.3828502,269.4335290,115.0456782,0.0$, $269.7453226,477.0745943,736.9396235,269.8511828,114.7320469,0.0$, $270.1650127,470.8914197,734.6411791,270.2672561,114.4139867,0.0$, $270.5830957,464.8539542,732.3849334,270.5830635,114.0904646,0.0$, $271.0009074,458.9465226,730.1136926,271.0994002,113.7607971,0.0$, $271.4192565,453.1591326,727.7926236,271.5168213,113.42445 \equiv 4,0.0$, $271.8387078,447.4845193,725.3990541,271.9357592,113.0809840,0.0$, $272.2597008,441.9163842,722.9176332,272.3565760,112.7299633,0.0$, $272.6826034,436.4493543,720.3378512,272.7795905,112.3709912,0.0$,

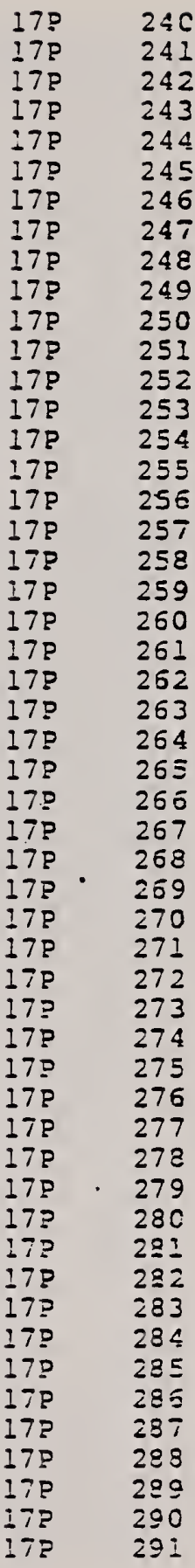




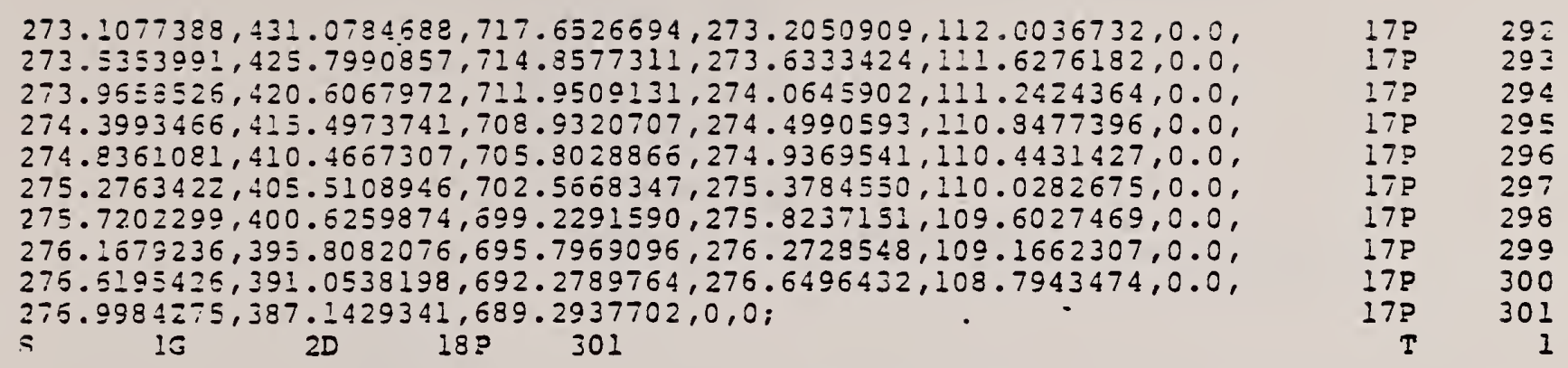

$3.4-11$ 
.

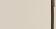

$\because$

. 


\subsubsection{Line}

- Case 1 (Figures 3.3.5-1 and 3.3.5-2) tests the following:

- horizontal lines

- vertical lines

- lines at angles

- 4 connected lines

- fonted lines (solid, dashed, phantom and centerline)

- Case 2

This is identical to Case 1 except lines are in 3-D parallel to a plane with rotation $\left(89^{\circ}, 89^{\circ}, 89^{\circ}\right)$ is used per section 3.0

- Case 3 (Figures 3.3.5-3 and 3.3.5-4) tests the following:

- long vertical, horizontal and angled lines (length $>1000$ in.)

- short vertical horizontal and angled lines (length $\leqslant .001$ in.)

Case 4

This case tests lines in 3-D

- long lines (length $>1000$ in)

- short lires (length $\leq .001 \mathrm{in}$ ) 


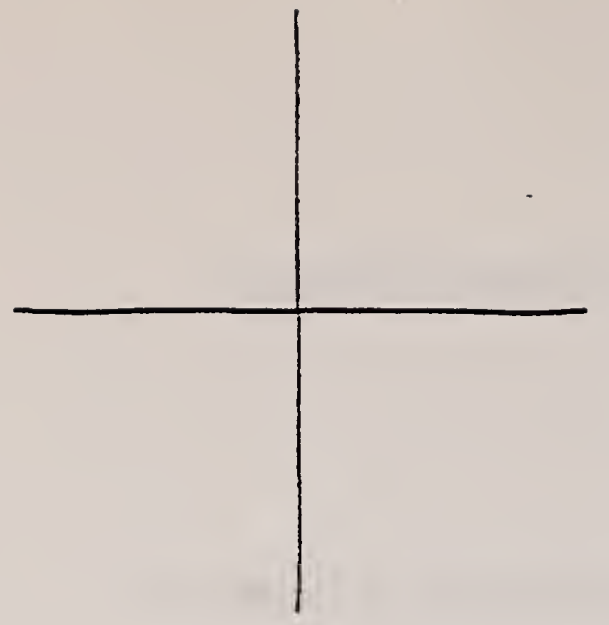

\begin{tabular}{l}
$u$ \\
$u$ \\
$z$ \\
\hline 1 \\
0 \\
$w$ \\
5 \\
$u$ \\
2 \\
2 \\
0 \\
0 \\
+
\end{tabular}
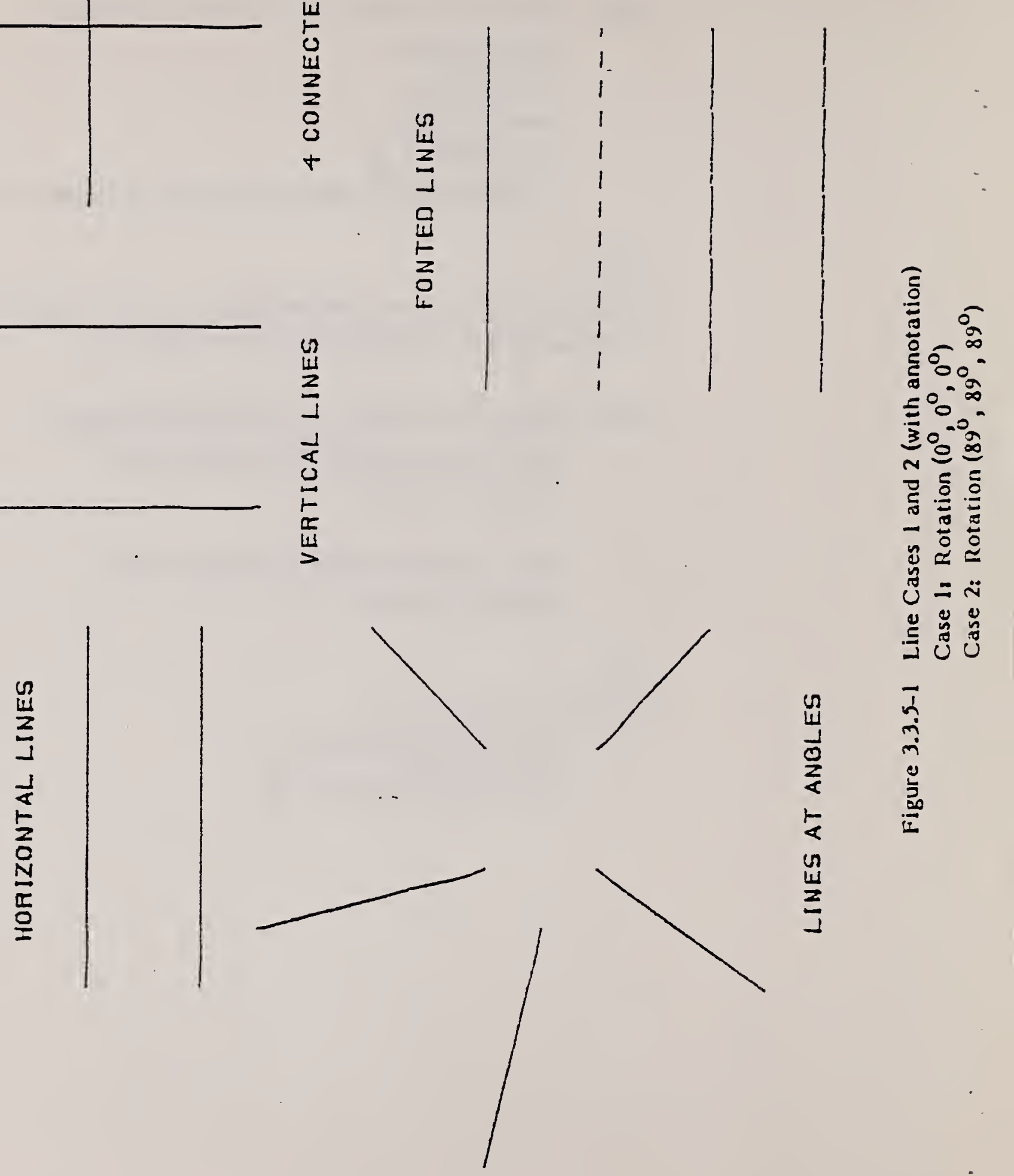


$$
\pm
$$




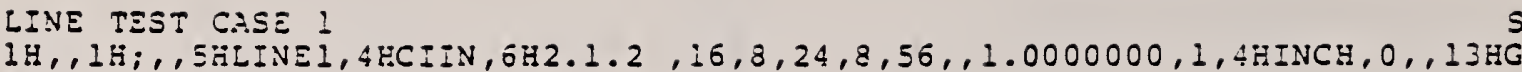
810713 .191724, , i;

$\begin{array}{ccccccc}8107131191724, \ldots & 1 & 1 & 0 & 0 & 0 \\ 110 & 0 & 0 & 1 & 0 & 0 & 0 \\ 110 & 2 & 1 & 1 & 0 & 0 & 0 \\ 110 & 0 & 0 & 1 & 0 & 0 & 0 \\ 110 & 3 & 1 & 1 & 0 & 0 & 0 \\ 110 & 0 & 0 & 1 & 0 & 0 & 0 \\ 110 & 4 & 1 & 1 & 0 & 0 & 0 \\ 110 & 0 & 0 & 1 & 0 & 0 & 0 \\ 110 & 5 & 1 & 1 & 0 & 0 & 0 \\ 110 & 0 & 0 & 1 & 0 & 0 & 0 \\ 110 & 6 & 1 & 1 & 0 & 0 & 0 \\ 110 & 0 & 0 & 1 & 0 & 0 & 0 \\ 110 & 7 & 1 & 1 & 0 & 0 & 0 \\ 110 & 0 & 0 & 1 & 0 & 0 & 0 \\ 110 & 8 & 1 & 1 & 0 & 0 & 0 \\ 110 & 0 & 0 & 1 & 0 & 0 & 0 \\ 110 & 9 & 1 & 1 & 0 & 0 & 0 \\ 110 & 0 & 0 & 1 & 0 & 0 & 0 \\ 110 & 10 & 1 & 1 & 0 & 0 & 0 \\ 110 & 0 & 0 & 1 & 0 & 0 & 0 \\ 110 & 11 & 1 & 2 & 0 & 0 & 0 \\ 110 & 0 & 0 & 1 & 0 & 0 & 0 \\ 110 & 12 & 1 & 3 & 0 & 0 & 0 \\ 110 & 0 & 0 & 1 & 0 & 0 & 0 \\ 110 & 13 & 1 & 4 & 0 & 0 & 0 \\ 110 & 0 & 0 & 1 & 0 & 0 & 0 \\ 110 & 14 & 1 & 1 & 0 & 0 & 0 \\ 110 & 0 & 0 & 1 & 0 & 0 & 0 \\ 110 & 15 & 1 & 1 & 0 & 0 & 0 \\ 110 & 0 & 0 & 1 & 0 & 0 & 0 \\ 110 & 16 & 1 & 1 & 0 & 0 & 0 \\ 110 & 0 & 0 & 1 & 0 & 0 & 0 \\ 110 & 17 & 1 & 1 & 0 & 0 & 0 \\ 110 & 0 & 1 & 0 & 0 & 0 \\ 110 & 0 & 0 & 1 & 0\end{array}$

$110,-3.0000000,5.0000000,0.0,-2.0000000,5.0000000,0.0,0,0$; $110,-8.0000000,3.0000000,0.0,-2.0000000,3.0000000,0.0,0,0$ : $110,0.0,2.0000000,0.0,0.0,7.0000000,0.0,0,0$;

$110,3.0000000,2.0000000,0.0,3.0000000,7.0000000,0.0,0,0$;

$110,-4.0000000,-2.0000000,0.0,-2.0000000,0.0,0.0,0,0$;

$110,-4.0000000,-4.0000000,0.0,-2.0000000,-6.0000000,0.0,0,0$; $110,-6.0000000,-4.0000000,0.0,-3.0000000,-7.0000000,0.0,0,0$;

$110,-7.0000000,-3.0000000,0.0,-11.0000000,-2.0000000,0.0,0,0$; $\begin{array}{llllll}0 & 0 & 0 & 0 & 0\end{array}$ $\begin{array}{lllllllll}0 & 0 & 0 & 0 & 0\end{array}$ $\begin{array}{ccc}0 & 0 & 0 \\ 0 & 0 & 0\end{array}$ $\begin{array}{lllll}0 & 0 & 0 & 0 & D\end{array}$ $\begin{array}{lllll}0 & 0 & 0 & 0 & D\end{array}$ OD $\begin{array}{lllll}0 & 0 & 0 & 0 & D\end{array}$ $\begin{array}{lllll}0 & 0 & 0 & 0\end{array}$ OD $\begin{array}{lllll}0 & 0 & 0 & 0 & D\end{array}$

$O D$ $\begin{array}{lllll}0 & 0 & 0 & 0 & D\end{array}$ $\begin{array}{llllll}0 & 0 & 0 & 0 & D\end{array}$ OD $\begin{array}{lllll}0 & 0 & 0 & 0 & 0\end{array}$ $\begin{array}{lllll}0 & 0 & 0 & 0 & D\end{array}$ OD $\begin{array}{lllll}0 & 0 & 0 & 0 & D\end{array}$ $\begin{array}{lllll}0 & 0 & 0 & 0 & 0\end{array}$ $\begin{array}{lllll}0 & 0 & 0 & 0 & D\end{array}$ $\begin{array}{lll}0 & 0 D & 26\end{array}$ $\begin{array}{llllll}0 & 0 & 0 & 0 & D & 27\end{array}$ $\begin{array}{lllllll}0 & 0 & 0 & 0 & D & & 29\end{array}$ $\begin{array}{llllll}0 & 0 & 0 & 0 & 0 & 30\end{array}$ $\begin{array}{lllll}0 & 0 & 0 & 0 & 32\end{array}$ $\begin{array}{llllll}0 & 0 & 0 & 0 & D & 33\end{array}$ OD $\quad 34$ $110,-5.0000000,-2.0000000,0.0,-7.0000000 ; 2.0000000,0.0,0,0$; $110,2.0000000,-2.0000000,0.0,8.0000000,-2.0000000,0.0,0,0$; $110,2.0000000,-4.0000000,0.0,8.0000000,-4.0000000,0.0,0,0$; $110,2.0000000,-5.0000000,0.0,8.0000000,-5.0000000,0.0,0,0$; $110,2.0000000,-8.0000000,0.0,8.0000000,-8.0000000,0.0,0,0$; $110,8.0000000,8.0000000,0.0,8.0000000,5.0000000,0.0,0,0$; $110,5.0000000,5.0000000,0.0,8.0000000,5.0000000,0.0,0,0$;. 
$110,8.0000000,2.0000000,0.0,8.0000000,5.0000000,0.0,0,0 ;$ $: 10,11.0000000,5.0000000,0.0,8.0000000,5.0000000,0.0,0,0$; 
LINE TEST CASE 2

$1 \mathrm{H},, 1 \mathrm{H} ;$, , 5HIINE2, 4HCIIN , 6H2.1.2, $16,8,24,8,56, .1 .0000000,1,4 \mathrm{HINCH}, 0,13 \mathrm{HG}$ $810713.191729, \ldots ;$

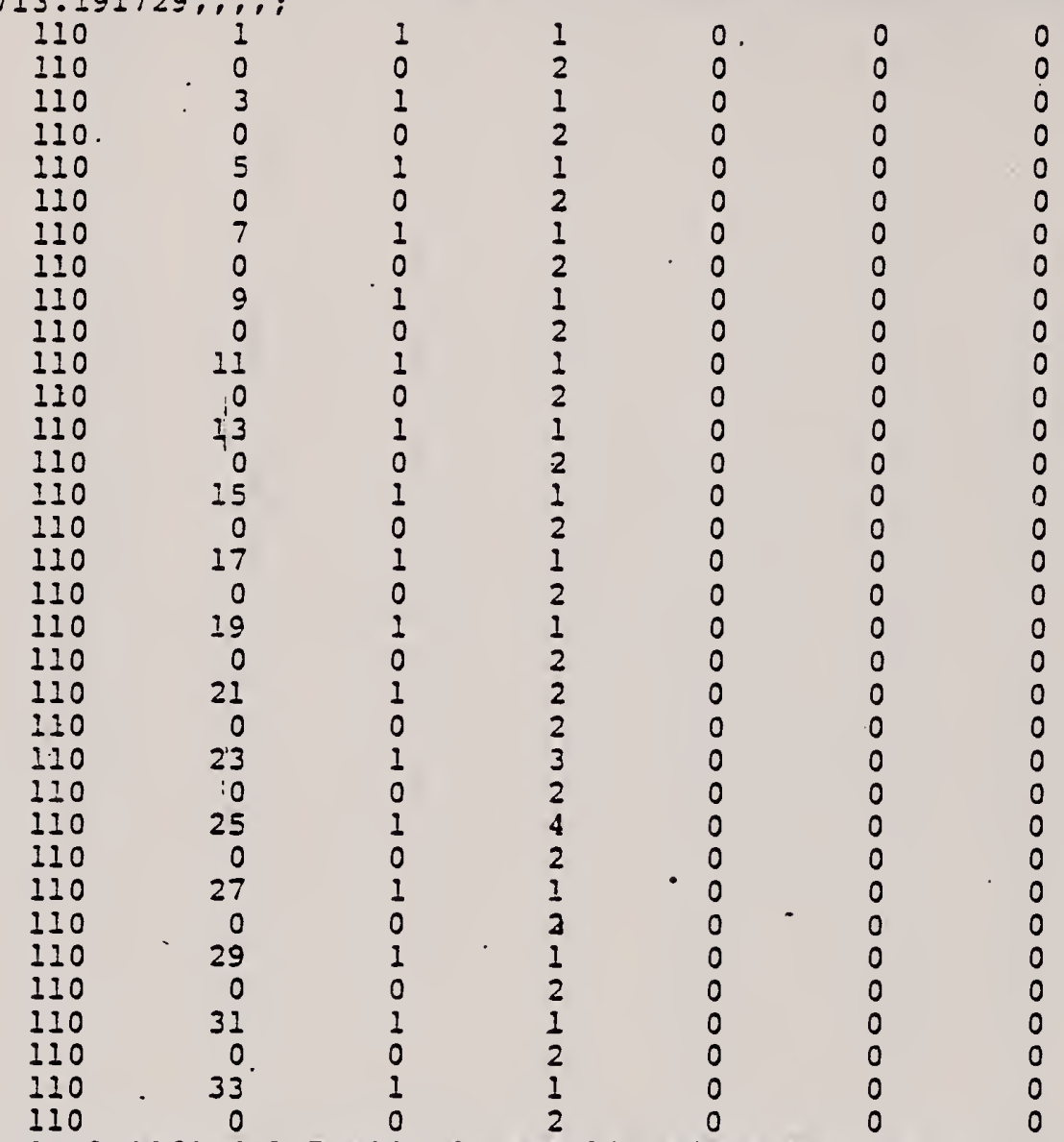

$110,-3.8349369,6.1070509,-6.0825319, .6650635,4.8080130$, $\begin{array}{llllll}0 & 0 & 0 & 0 & 0\end{array}$ $\begin{array}{lllll}0 & 0 & 0 & 0 & 0\end{array}$ $\begin{array}{llllll}0 & 0 & 0 & 0 & 0 D\end{array}$ $\begin{array}{lllll}0 & 0 & 0 & 0 & D\end{array}$ $\begin{array}{lllll}0 & 0 & 0 & 0 & D\end{array}$ 0000 OD 10 OD 12 $\begin{array}{lllllllll}0 & 0 & 0 & 0 & 0 & 13\end{array}$ $\circ 000$ OD 14 $\begin{array}{llllll}0 & 0 & 0 & 0 & D & 16\end{array}$ $\begin{array}{llllrl}0 & 0 & 0 & 0 & D & 18 \\ & & & \end{array}$ $\begin{array}{llllll}0 & 0 & 0 & 0 & 0 & 20 \\ 0 & & 21\end{array}$ OD 22 $\begin{array}{llllllll}0 & 0 & 0 & 0 & D & 23\end{array}$ $\begin{array}{rrrrrr}0 & 0 & 0 & 0 & D & 24\end{array}$ OD 26 $\begin{array}{llllll}0 & 0 & 0 & 0 & D & 2 T\end{array}$ $\begin{array}{llllll}0 & 0 & 0 & 0 & D & 28\end{array}$ $\begin{array}{lllll}0 & 0 D & 30\end{array}$ $\begin{array}{llll}0 & O D & 32\end{array}$ $\begin{array}{llllll}0 & 0 & 0 & 0 & D & 33\end{array}$ $-2.3325319,0,0$;

$110,-4.7009621,4.3570509,-5.6495194,-.2009619,3.0580127$, $-1.8995192,0,0$ :

$110, .8660254,1.7500001,-.4330127,3.0310991,6.1250000,-1.5155445$, 0,0 ;

$110,3.1160254,1.1004810,1.4419874,5.2810888,5.4754810, .3594556$, 0,$0 ;$

$110,-3.8660257,-.8839747,-2.0669875,-1.5000001, .4330127$,

$-1.2500001,0,0$;

$110,-4.7320509,-2.6339748,-1.6339748,-4.0980753,-4.8169875$, $.0490381,0,0$ :

$110,-6.2320509,-2.2009621,-2.8839748,-9.0310888,-4.3929491$, $-3.4844558,0,0$;

$110,-6.5490384,-1.1094556,-3.7254813,-9.1160259, .6315699$,

$\begin{array}{lr}\text { OD } & 34 \\ 1 P & 1 \\ 1 P & 2 \\ 3 P & \quad \\ 3 P & 4 \\ 5 P & 5 \\ 5 P & 6 \\ 7 P & 7 \\ 7 P & 8 \\ 9 P & 9 \\ 9 P & 10 \\ 11 P & 11 \\ 11 P & 12 \\ 13 P & 13 \\ 13 P & 14 \\ 15 P & 15\end{array}$


$-6.4419875,0,0$;

$110,-5.366025 \div,-.4509619,-3.3169875,-4.3839746,3.2555447$,

$-4.8080130,0,0$;

$110, .6339747,-2.1830127,1.6830128,5.1339750,-3.4820509$,

$5.4330130,0,0$;

$110,-.2320508,-3.9330130,2.1160254,4.2679496,-5.2320509$,

$5.8550259,0,0$;

$110,-1.0980762,-5.5830130,2.5490382,3.4019241,-6.9820509$,

$6.2990384,0,0$;

$110 .,-1.9541017,-7.4330130,2.9820509,2.5358987,-8.7320509$,

$6.7320514,0,0$;

$110,9.4641018,5.2679496,3.2679496,8.1550639,2.6429493,3.9174685$,

0,0 ;

$110,5.9150639,3.2924683,2.0424683,8.2650639,2.6429493,3.9174685$,

0,$0 ;$

$110,6.8660259, .0179492,4.5669875,8.1650639,2.6429493,3.9174685$,

0,$0 ;$

$110,10.4150639,1.9934303,5.7924685,8.1650639,2.6429493$,

$3.9174685,0,0$;

$1 \mathrm{G}$

$2 D$

$34 P$

34

$\begin{array}{rr}15 P & 19 \\ 17 P & 17 \\ 179 & 18 \\ 19 P & 19 \\ 19 P & 20 \\ 219 & 21 \\ 219 & 22 \\ 23 P & 23 \\ 23 P & 24 \\ 259 & 25 \\ 25 P & 25 \\ 279 & 27 \\ 279 & 28 \\ 29 P & 29 \\ 29 P & 30 \\ 319 & 31 \\ 31 P & 32 \\ 33 P & 33 \\ 33 P & 34 \\ 9 & 1\end{array}$



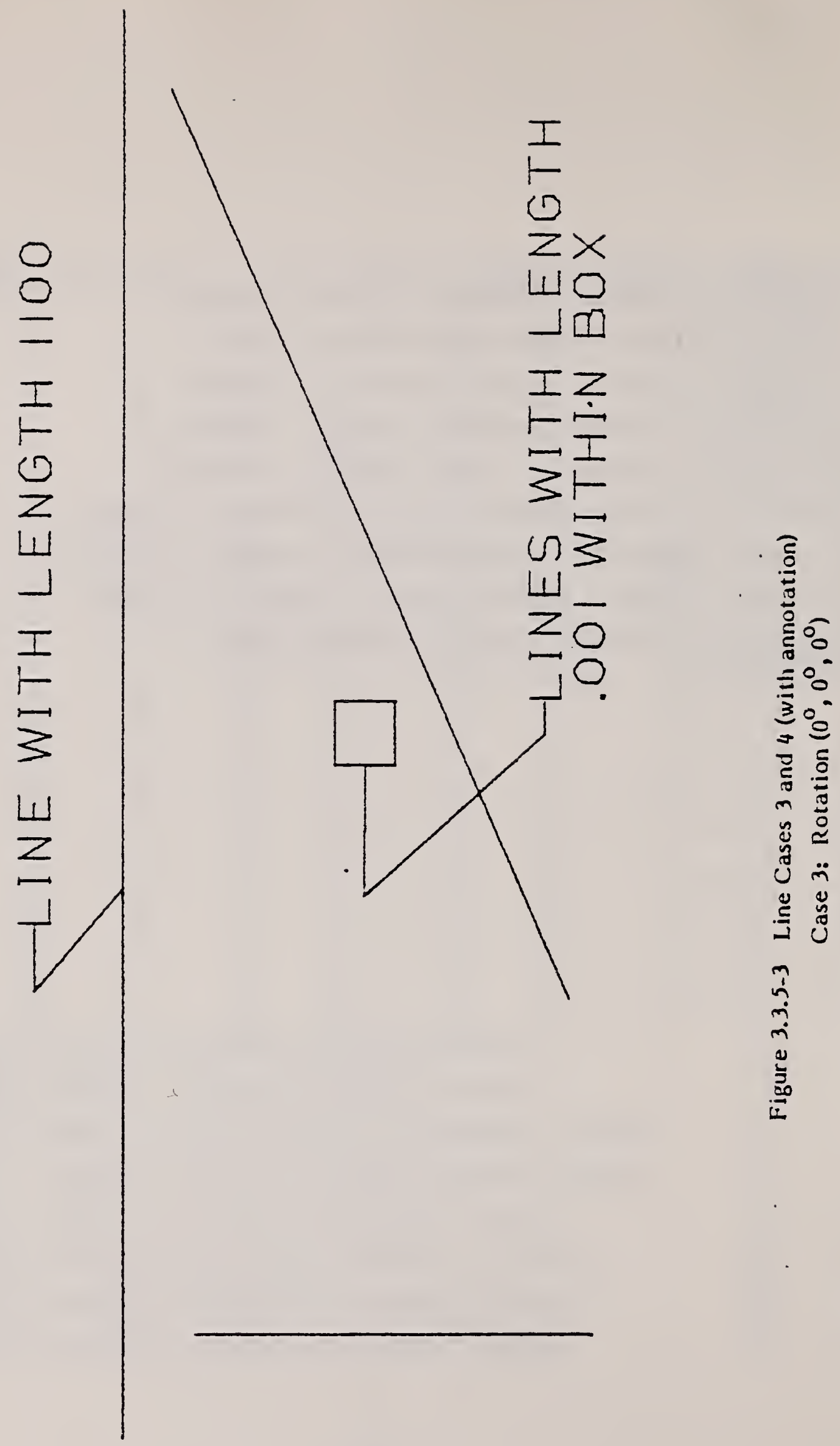


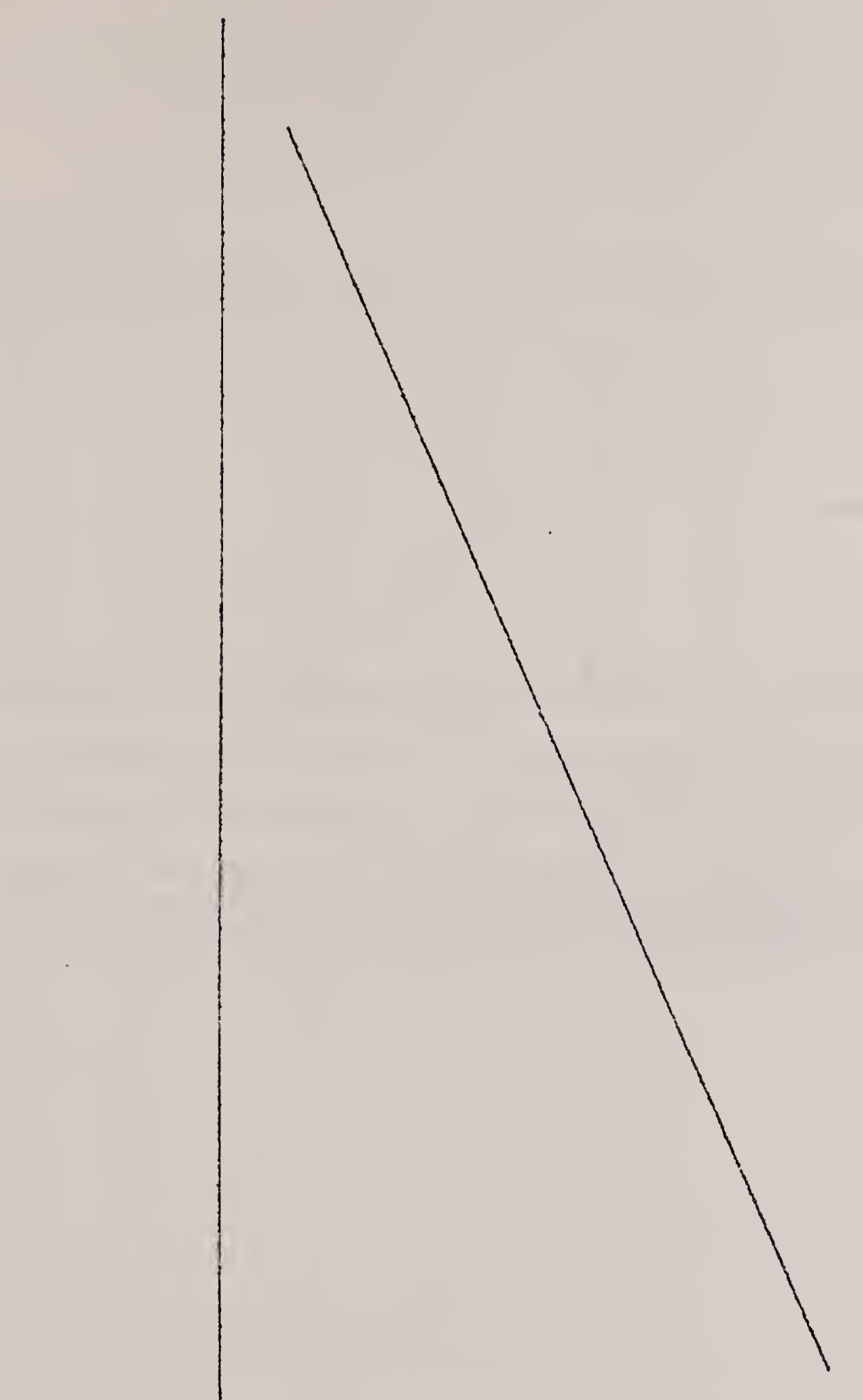



$3.5-9$ 

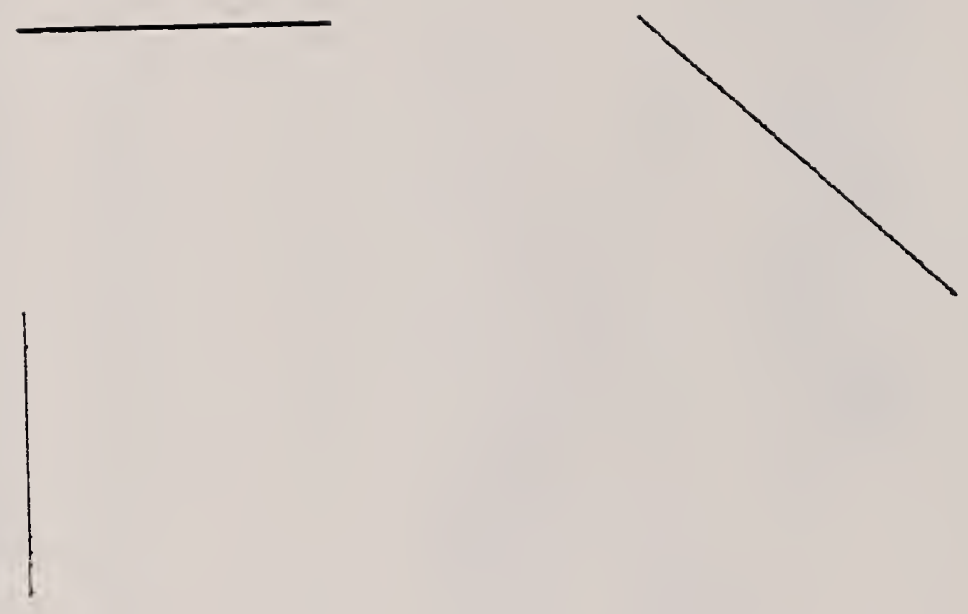

Figure 3.3.5-5 Line Cases 3 and 4 (magnified to show small lines) 
LINE TEST CASE 3

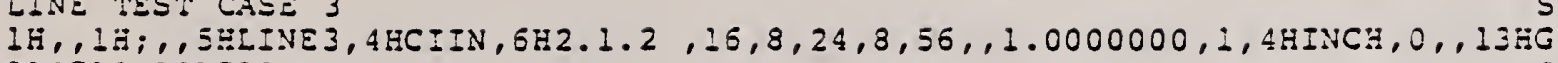
$810713.191733, \ldots$, ;

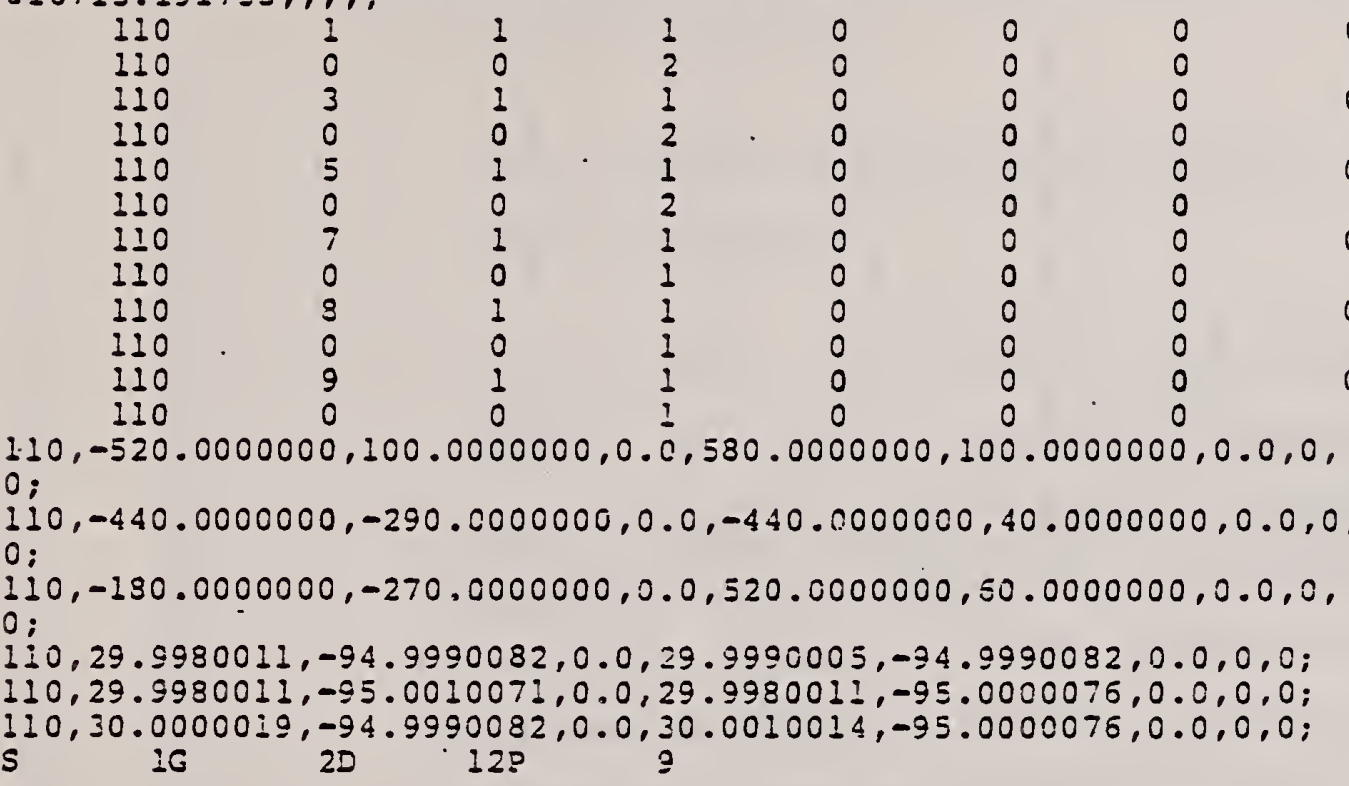

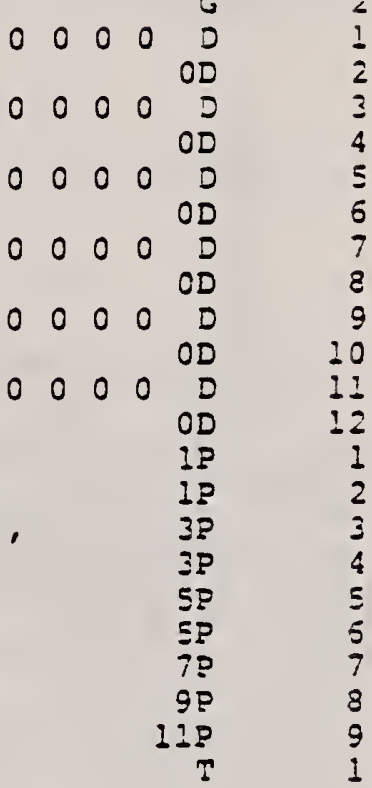


LIVE TEST CASE 4

$110,-4.7662959,-269.9583435,-179.9992523,1.2053807,59.9894714$, $519.9997559,0,0$;

$110,-1.6485848,-94.9846039,29.9982491,-1.6485845,-94.9846039$, $29.9992485,0,0$;

$110,-1.6486197,-94.9866028,29.9982491,-1.6486022,-94.9856033$, $29.9982491,0,0$;

$110,-1.6485842,-94.9846039,30.0002499,-1.6486013,-94.9856033$, $30.0012493,0,0$; 
- Case 1

Spline types of linear, cubic, Wilson-Fowler and Modified Wilson-Fowler are tested.

- Level 2 (Figures 3.3.6-1 and 3.3.6-2) contains a 2D and 3D cubic spline in model space.

- Level 3 (Figure 3.3.6-3) contains a Wilson-Fowler spline in mode! space.

- Level 4 (Figure 3.3.6-4) contains 3 Modified Wilson-Fowler splines.

- One in model space.

- One in non-model space with rotation $\left(0^{\circ}, 0^{\circ}, 2^{\circ}\right)$ per section 3.0.

- One in non-model space with rotation $\left(0^{\circ}, 0^{\circ}, 71^{\circ}\right)$ per section 3.0 .

- Case 2 (Figure 3.3.6-5)

- A uniform, cubic B-spline in model space 


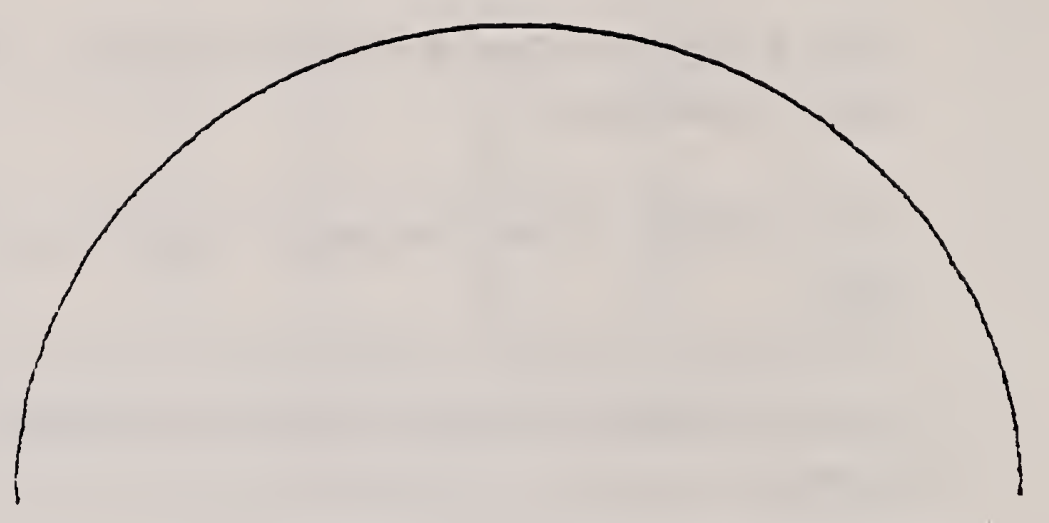

Figure 3.3.6-1 2D Cubic Spline (actual part)

Case 1: Rotation $\left(0^{\circ}, 0^{\circ}, 0^{\circ}\right)$

$3.6-2$ 


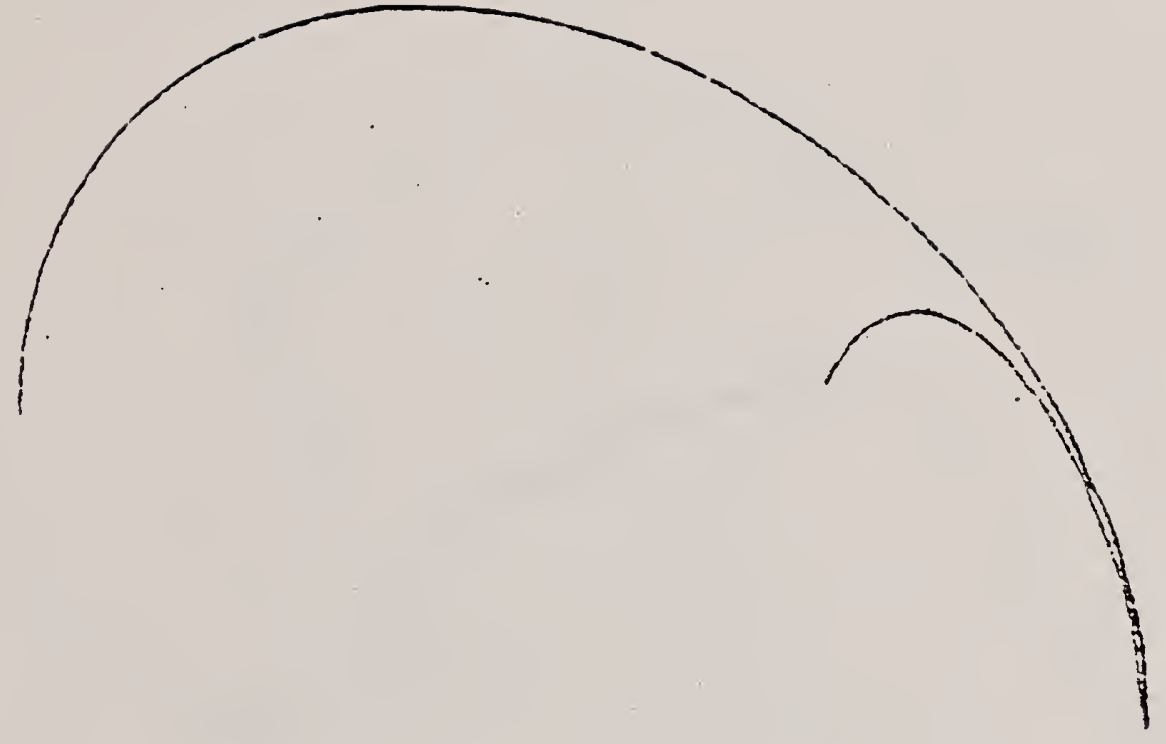

LUBIC SPLINE

Figure 3.3.6-2 3D Cubic Spline (actual part) Case 1: Rotation $\left(0^{\circ}, 0^{\circ}, 0^{\circ}\right)$ 


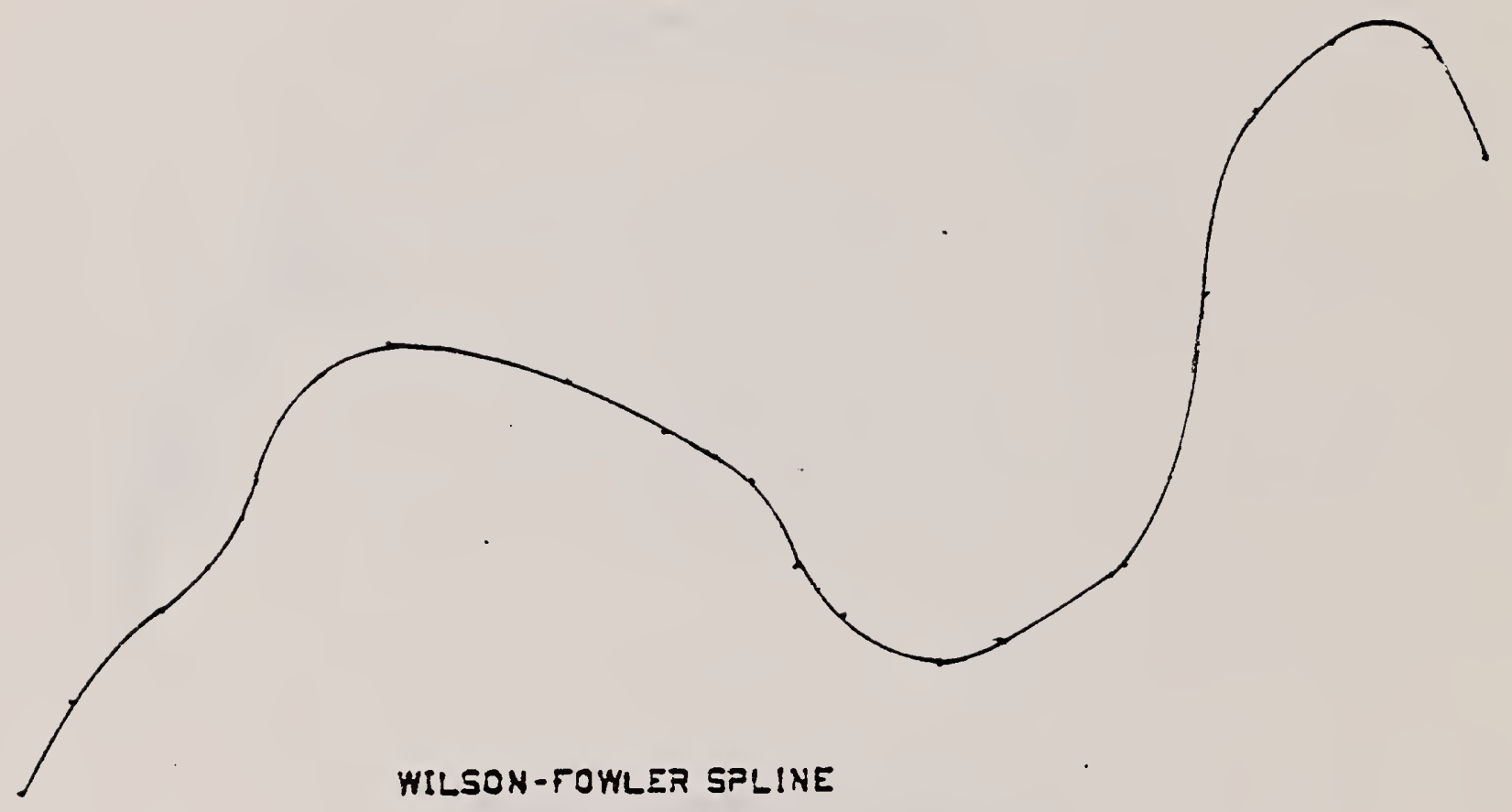

Figure 3.3.6-3 Wilson Fowler Spline (actual part) Case 1: Rotation $\left(0^{\circ}, 0^{\circ}, 0^{\circ}\right)$ 


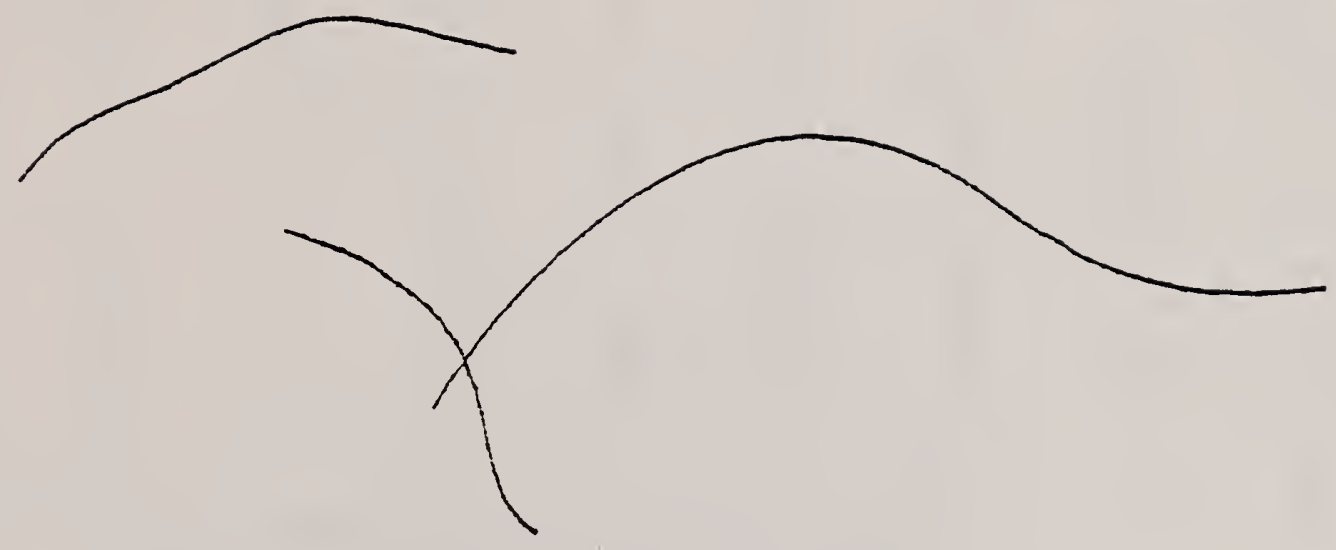

Figure 3.3.6-4 Modified Wilson Fowler Splines (actual part) Case 1: Rotation $\left(0^{\circ}, 0^{\circ}, 0^{\circ}\right)$ 


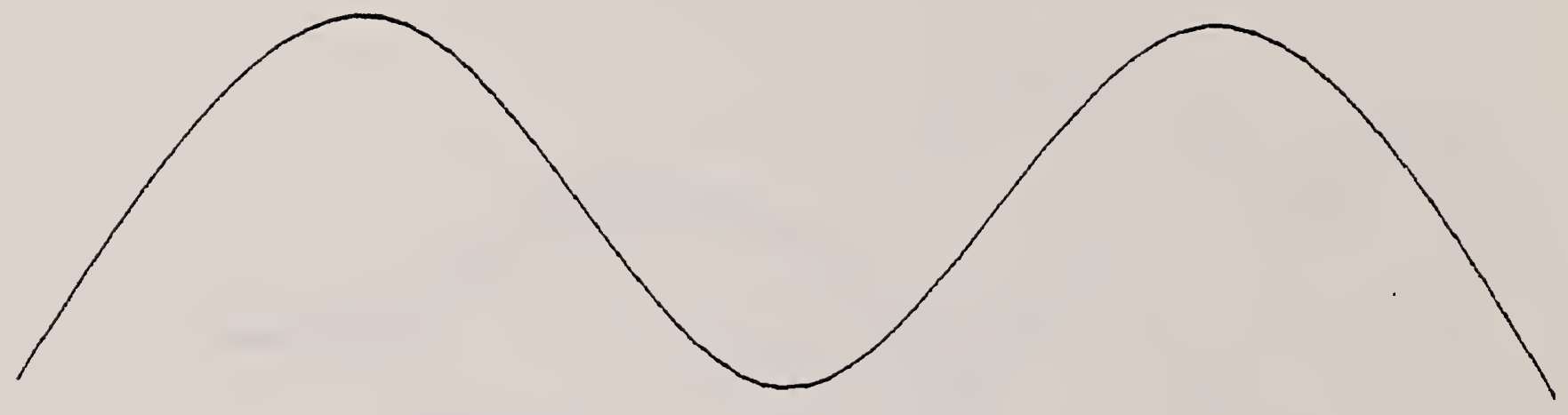

Figure 3.3.6-5 Uniform B-Spline (actual part)

Case 2: Rotation $\left(0^{\circ}, 0^{\circ}, 0^{\circ}\right)$ 
SPLINES TEST CASE I

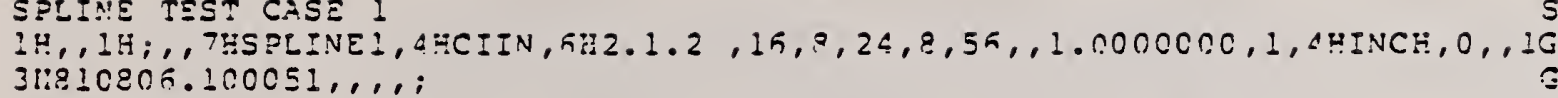

$\begin{array}{lrrrrrr}10206 & 120 & 1 & 1 & 0 & 0 & 0 \\ 124 & 0 & 1 & 1 & 0 & 0 & 0 \\ 112 & 3 & 1 & 1 & 3 & 0 & 1 \\ 112 & 0 & 0 & 39 & 0 & 0 & 0 \\ 112 & 42 & 1 & 1 & 4 & 0 & 1 \\ 112 & 0 & 0 & 15 & 0 & 0 & 0 \\ 124 & 58 & 1 & 1 & 0 & 0 & 0 \\ 124 & 0 & 0 & 2 & 0 & 0 & 0 \\ 112 & 90 & 1 & 1 & 4 & 0 & 7 \\ 112 & 0 & 0 & 13 & 0 & 0 & 0 \\ 124 & 72 & 1 & 1 & 0 & 0 & 0 \\ 124 & 0 & 0 & 2 & 0 & 0 & 0 \\ 112 & 75 & 1 & 1 & 4 & 0 & 11 \\ 112 & 0 & 0 & 13 & 0 & 0 & 0 \\ 112 & 88 & 1 & 1 & 2 & 0 & 1 \\ 112 & 0 & 0 & 38 & 0 & 0 & 0 \\ 112 & 125 & 1 & 1 & 2 & 0 & 1 \\ 112 & 0 & 0 & 57 & 0 & 0 & 0\end{array}$

$128,1.0000000,0.0,0.0,0.0,0.0,1.0000000,0.0,0.0,0.0,0.0$, $1.0,000000,0.0,0,0 ;$

$112,4,0,2,21,0.0,2.2350630,5.0644951,5.4787087,8.7147756$, $10.0509445,13.1859125,17.3100122,19.5460552,21.7821542, \ldots$ $24.0192222,25.4324357,27.5585037,29.9045717,22.1406397$, $34.3757075,28.4998133,42.5229120,44.0371324,16.2732004$, $47.5874140,50.9234819,0.0, .2863025, .0710517, .24825345-17,0.0$, $.9748828,-.0359808,-.174: 1257 \mathrm{E}-17,0.0,0.0,0.0,0.0,1.0000000$, $.6047539, .0552470,-.0070922,2.0000000, .0094597,-.0562470$, $.0070922,0.0,0.0,0.0,0.0,3.0000000, .7527220,-.0041021,-.0290070$,

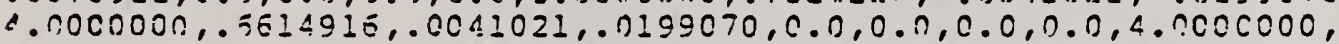
$.5209371,-.1154933, .0155419,5.0000000, .9030654, .0582346$, $-.0077710,0.0,0.0,0.0,0.0,5.0000000, .3421099,-.0121361, .0254525$, $7.0000000, .3459341, .0050031,-.0132263,0.0,0.0,0.0,0.0,6.0000000$, $.7136276, .0934254$, . .CS55212,0.0000000,.8082127,-.1868508. $.0112424, C .0,0.0,0.0,0.0,8.0000000,1.0650029,-.0320152, .0020733$, 2n. $0000000, .1445060,-.1280 \pi 49, . n 022932,0.0,0.0,0.0,0.0$,

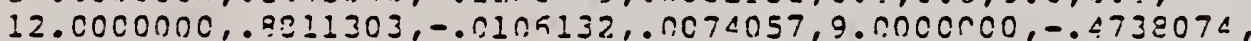
$-.0212254, .0148115,0.0,0.0 .0 .0,0.0,14.0000000, .0417528, .0301504$, $-.0275739,8.0000000,-.3465624, .0723018,-.0551479,0.0,0.0,0.0$, $0.0,25.0000000, .5710102,-.2504908, .0572576,7.0000000,-.7825280$, $-.1252499,00336338,0.0,0.0,0.0,0.0,17.0000000, .5552027, .1543224$, $-.0458300,5.0000 \mathrm{c} \cap 0,-.8479300, .1643924,-.045 \pi 309, \mathrm{c} . \mathrm{C}, 0.0,0.0$,

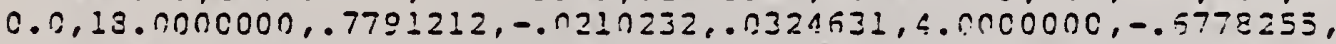
-.0420454,.0640251,0.0,0.0,0.0,0.n, 20.0000000,1.0537783,

$-.1434500, .0319702,3.0500000, .0985104, .2989201,-.0539405,0.0$, $0.0,0.0,0.0,22.0000000, .8709258, .0651419,-.0252412,4.0000000$, $.8752144,-.1303237, .0524024,0.0,0.0,0 . n, 0.0,24.06 n c 000, .0120257$, -. Z535401,.04C2522,5.0000000,.7115211,.1253201,-.0201341,0.0,

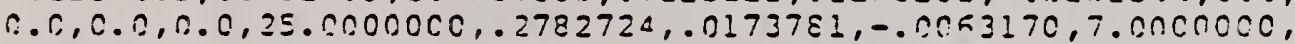
$.0512093,-.00424 A 5, .0015792,0.0,0.0,0.0,0.0,25.0000000, .0004: 04$, 
$-.0508531, .0231806,11.0000000,1.0050238, .0152158,-.0057051,0.0$, ก.c, c.0, ..C, $27.0 n 00000, .5225: 73, .1104887,-.0553475,15.0 n 0000 n$, $.7315653,-.1104397, .0558475,0 . n, n .0, n .0, c . c, 28.000 n c 0 n, .6560542$, $-.1453434, .1111193,15.0000000, .0030305, .29252 \div 8,-.2222337,0.0$, c.c.0.0, ..0,30.0000nco,.0.479082,--.1224887,.0151578,17.0000000, $-.5552254,-.1224687, .0151 \leq 78,0.0,0.0,0.0,0.0,31.0000000$, $.5002 n n 2,-.0684177, .7647502 E-17,16.0000000,-.9179339,-.0342090$, $.3723801 E-17,0.0,0.0,0.0,0.0,31.0000000, .5002002,-.1368354$, $.4468551 \mathrm{a}-15,15.0000000,-.8170339,-.0584177, .2234221 \equiv-15,0.0$, $0.0,0.0,0.0,0,0$;

$112,5,0,2,9,1.2719091,1.4799903,1.9550990,2.3190992,2.0990975$, $3.70009 .3,4.2539980,4.9350981,5.4390077,5.0390071,1.2710991$, $1.0000000,0.0,0.0,2.3359985,5.5851707,-25.0 \leq 23555,49.4484100$, $0.0,0.0,0.0,0.0,1.4790993,1.0000000,0.0,0.0,3.0230383,2.1595475$, $-2.0233784,1.4731183,0.0,0.0,0.0,0.0,1.8559090,1.000000 c, 0 . c$, $0.0,3.5319976,1.2727575,-.5553737, .1929117,0.0,0.0,0.0,0.0$, $2.3190992,1.0000000,0.0,0.0,4.1190980, .2722259,-.2455377$,

$-.0042488,0.0,0.0,0.0,0.0,2.0999075,1.0000000,0.0,0.0,4.5519981$, $.3954013,-.3492873,-. c 421438,0.0,0.0,0.0,0.0,3.7929983$, $1.0000000,0.0,0.0,4.6230077,-.2433745,-.4788300, .0001501,00.0$, $0.0,0.0,0.0,4.2639980,1.0000000,0.0,0.0,4.4070081,-.5875252$, -.4715988,.5379271, c.n,0.0,0.0,0.0,4.8159981, 1.0000000,0.c,n.0, $3.0919877,-.5252437, .4901187,-.1345374,0.0,0.0,0.0,0.0$, $5.4359977,1.0000000,0.0,0.0,3.7590074,-.1707328, .2538025$, $-.0228200,0.0,0.0,0.0,0.0,5.4399977,1.0000000,0.0,0.0,3.7509974$, $-.1707328, .5 n 75050,-.1360200,0.0,0.0,0.0,0.0,0,0$; $124, .0993906, .0348992,0.0,0.0,-.0348992, .0993905,0 . n, 0.0, n . n$, $0.0 .1 .0000000,0.0,0,0$ :

$112,5,0,2,7,-.9707423,-.8515917,-.5198555,-.2815025, .2599888$, $.9277000,1.5857108,1.9240549,-.0707423,1.0000000,0.0,0.0$, $3.5215421,5.1570097,-18.3800507,45.7533645,0.0,0.0,0.0,0.0$, $-.9515017,1.0000000,0.0,0.0,3.9538841,2.7657005,-3.7920985$, $4.0551001,0.0,0.0,0.0,0.0,-0.5109555,1.0000000,0.0,0.0,4.4425155$, $1.5610451,-1.5373434, .4771501,0.0,0.0,0.0,0.0,-.2815026$, $1.0000000,0.0,0.0,4.8373642, .7219237,-.0007566, .0209391,0.0,0 . n$, $0 . n, 0 . n, .2598883,1.000 n 000,0.0,0.0,5.1005287, .4246470, .3520545$, $-.5858209,0.0,0.0,0.0,0.0, .9277909,1.0000000,0.0,0.0,5.4075537$, $.1951118,-.7513812, .3232574,0.0,0.0, n .0,0 . n, 1.5857108,1.0000000$, $0.0,0.0,5.3108318,-.2958344, .0445310, .3499294,0.0,0.0,0 . c, 0.0$, $1.5257108,1.0000000,0.0,0.0,5.3139313,-.2953844, .0801510$, $2.0095754,0.0,0.0,0.0,0.0,0,0$; $124, .3755521, .0455184,0.0,0.0,-.0455184, .3255421,0.0,0.0,0.0$, $0.0,1.0000000,0.0,0,0:$

$112,5, n, 2,7,-3.7373052,-3.4700454,-3.1087920,-2.0051004$, $-2.5313225,-2.3129533,-1.0243036,-1.7975037,-3.7373552$, $1.0000000,0.0,0.0,2.2780337,1.3075010,-1.5699446,1.2024587, r .0$, $0 . n, c .0,0.0,-3.4709454,1 . \operatorname{con} 0000,0.0, n .0,2.5517551, .910521=$, $-.5787245, .3479272,0.0,0.0,0.0,0.0,-3.1987929,1 . \operatorname{con} 00 \mathrm{co}, 0 . n, 0.0$, $2.7412710, .5253552,-.4534544,-.3350705,0 . n, n, n, 0.0,0.0$. $-2.8851004,1 . n 00 n c 00,0.0, n .0,3.55129 ? 9, .1445410,-.7457789$,

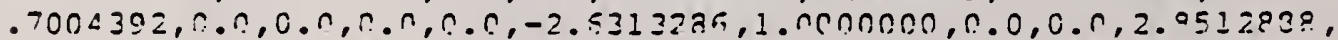
$-. r 990740,-.2000753, .1580001,0.0,0.0,0 . n, 0 . n,-2.3128532$,

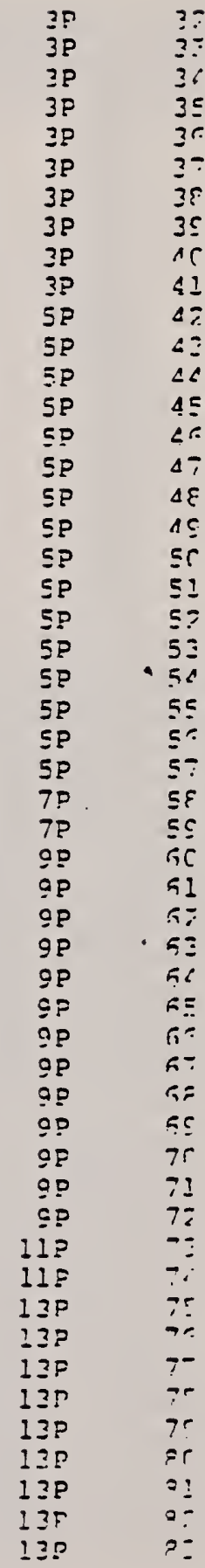


$1.0000000,0.0,0.0,2.8049655,-.1716579,-.0077140, .9620458,0.0$, $0.0,0.0,0.0,-1.9248935,1.0000000,0.0,0.0,2.7933846, .2557570$, $1.0833561,3.5833868,0.0,0.0,0.0,0.0,-1.9248936,1.0000000,0.0$, $0.0,2.7933846, .2567570,2.1667123,22.1003208,0.0,0.0,0.0,0.0,0,0$; $112,3,0,2,20,0.0, .2255125, .6249973,1.0244054,1.4238676$, $1.8236292,2.0955623,2.4947605,2.8943290,3.2938080,3.6934843$, $3.9653941,4.3649945,4.6366426,4.9085522,5.1804630,5.4525724$, $5.7243701,5.9962628,6.2681359,6.2681369,-2502.6000977, .0384577$, $0.0, .4358998,579.0290527,1.0000929,0.0,=.0379968,0.0,0.0,0.0$, $0.0,-2502.5864258, .1049619, .2949025,-.0551033,599.2541504$, $.0942958,-.0257063,-.0450447,0.0,0.0,0.0,0.0,-2502.5009766$, $.3141528, .2288803,-.0065292,599.6442871, .9522075,-.0796767$, $-.0367155,0.0,0.0,0.0,0.0,-2502.3393555, .4939055, .2210548$, $-.0253096,600.0096436, .8709639,-.1236323,-.0353520,0.0,0.0,0.0$, $0.0,-2502.1083934, .6583957, .1907242,-.0340757,600.3355713$, $.7552277,-.1650477,-.0284184,0.0,0.0,0.0,0.0,-2501.8168945$, $.7945473, .1498573,-.0290074,600.6091309, .6088442,-.2001294$, $-.0186703,0.0,0.0,0.0,0.0,-2501.5903320, .8696149, .1261935$, $-.0394897,600.7595215, .4958584,-.2153607,-.0229202,0.0,0.0,0.0$, $0.0,-2501.2255859, .9514881, .0789009,-.0414603,600.9217529$, $.3134350,-.2416121,-.0056566,0.0,0.0,0.0,0.0,-2500.8354492$, $.9946827, .0292022,-.0407993,601.0080565, .1176455,-.2483927$, $-.0024372,0.0,0.0,0.0,0.0,-2500.4360352, .9984813,-.0196932$, $-.0447514,601.0152588,-.0819767,-.2513135, .0081693,0.0,0.0,0.0$, $0.0,-2500.0429688, .9612936,-.0733514,-.0293878,600.9428711$, $-.2789499,-.2415183, .0160201,0.0,0.0,0.0,0.0,-2499.7875977$, $.9148853,-.0973239,-.0451330,600.8494873,-.4067390,-.2284502$, $.0178055,0.0,0.0,0.0,0.0,-2499.4404297, .8154834,-.1514293$, $-.0194162,600.6516113,-.5807870,-.2071050, .0343843,0.0,0.0,0.0$, $0.0,-2499.2304688, .7289141,-.1672525,-.0385164,600.4792480$, -. $5856945,-.1790837, .0233108,0.0,0.0,0.0,0.0,-2499.0454102$, $.5294159,-.1986714,-.0102541,600.2800293,-.7779132,-.1600684$, $.0409681,0.0,0.0,0.0,0.0,-2498.8891602, .5190996,-.2070361$, $-.0457711,600.0574951,-.8558749,-.1266494, .0282874,0.0,0.0,0.0$, $0.0,-2498.7641602, .3962595,-.2444003, .0340857,599.8157959$. $-.9185164,-.1035576, .0474737,0.0,0.0,0.0,0.0,-2498.6738281$, $.2709590,-.2165062,-.1235455,599.5594482,-.9642986,-.0648479$, $.0368954,0.0,0.0,0.0,0.0,-2498.6186523, .1257722,-.3173796$, $.3891256,599.2932129,-.9913694,-.0347532, .0426094,0.0,0.0,0.0$, $0.0,-2498.6000977,0.0,0.0,0.0,599.0219727,0.0,0.0,0.0,0.0,0.0$, $0.0,0.0,-2498.5000977,0.0,0.0,0.0,599.0219727,0.0,0.0,0.0,0.0$, $0.0,0.0,0.0,0,0$;

$112,3,0,3,31,0.0, .2719033, .5714031, .9433063,1.3428058,1.7423051$, $2.0142084,2.4137074,2.8132071,3.2127060,3.6122057,4.0117048$, $4.4112045,4.8107045,5.2102045,5.6097035,5.8816074,6.1535108$, $6.2711145,6.5753975,6.8760794,7.0818746,7.2870688,7.4922637$, $7.7935460,8.0948230,8.3000226,8.5052167,8.7104114,8.9156066$, $9.0052996,9.0052996, .9838046, .0348794,0.0, .3244740,2.1824389$, $.8564018,0.0,-.1257731,1.9159014, .5176950,0.0, .1431206, .9993111$, $.1068459, .2646767,-.0627361,2.4127488, .8282831,-.1034142$, $-.0215909,2.0595417, .5494392, .1167449,-.0542858,1.0807383$, $.2882843, .1894875, .0103111,2.7257662, .7353175,-.1292908$,

$13 P$

135

139

$13 P$

$15 \mathrm{P}$

$15 P$

$15 P$

$15 P$

$15 ?$

$15 P$

$1 \equiv P$

15?

$15 ?$

$15 P$

15 ?

$15 P$

$15 P$

$15 \mathrm{P}$

15 P

15 P

$15 P$

\5P

$15 P$

$15 \mathrm{P}$

isP

15 P

$15 \mathrm{P}$

$15 \mathrm{P}$

158

$15 P$

158

15 ?

$15 P$

$15 P$

$15 P$

$15 P$

$15 P$

$15 \mathrm{P}$

$15 \mathrm{P}$

$15 \mathrm{P}$

$1 \equiv P$

$15 F$

$17 ?$

170

$17 P$

17 ?

178

$17 ?$

17?

$17 P$

$17 ?$

:75 $\varepsilon \div$

85

86

87

89

80

90

91

92

93

94

95

96

97

98

99

100

101

102

103

104

105

206

$\lcm{07}$

108

109

110

111

112

113

114

115

115

117

118

110

120

121

122

123

124

125

125

127

122

129

130

131

132

133

134

135 
$-.0301380,2.2942138, .6167263, .0516833,-.0105403,1.1733401$, $.3936158, .1978983,-.0232532,2.9155369, .6583239,-.1538747$, $-.0251188,2.4655128, .6424942, .0430856,-.0305710,1.3606913$, $.5405028, .1700295,-.0254296,3.1523132, .5228725,-.1851781$, $-.0188928,2.7271161, .6622822, .0064463,-.0285366,1.5021132$, $.6638015, .1383537,-.0250792,3.3304415, .3658696,-.2078211$, $-.0109925,2.9909067, .6537694,-.0277548,-.0230526,1.7923276$, $.7334768, .1178964,-.0342911,3.4143372, .2504171,-.2167878$, $-.0080331,3.1661534, .6335632,-.0465591,-.0275076,2.1019807$, $.8112573, .0767986,-.0346088,3.4792671, .0733578,-.2264155$, $.0010430,3.4100766, .5831921,-.0795268,-.0224650,2.4361281$,

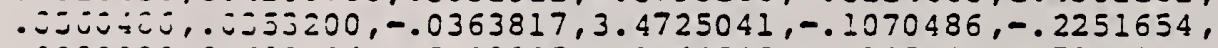
$.0080932,3.6289368, .5088935,-.1064523,-.0195787,2.7814360$, $.8658497,-.0082834,-.0359517,3.3943181,-.2830803,-.2154657$, $.0155069,3.8140011, .4144641,-.1299173,-.0150304,3.1241279$, $.8430176,-.0513715,-.0342548,3.2478280,-.4478126,-.1968807$; $.0215453,3.9578862, .3034637,-.1479312,-.0103413,3.4505291$, $.7855659,-.0924378,-.0312447,3.0388789,-.5948040,-.1710587$, $.0290598,4.0548506, .1803155,-.1603252,-.0042977,3.7476172$, $.0567482,-.1298845,-.0256683,2.7758074,-.7175612,-.1362135$, $.0253075,4.1010246, .0501580,-.1654760,-.00 .13400,4.0035381$, $.5802017,-.1618465,-.0222198,2.4691105,-.3135644,-.1040898$, $.0556307 .4 .0945673,-.0825989,-.1670819, .0136968,4.2080812$, $.4402475,-.1884769,-.0124730,2.1310258,-.8700961,-.0374164$, $-.0377731,4.0357361,-.2095393,-.1506663,-.0166505,4.3530836$, $.2836828,-.2034258,-.0278086,1.7750432,-.9180774,-.0826873$, $.5315390,3.9268775,-.3379936,-.1706218, .2017731,4.4146194$, $.1668905,-.2251096, .0734518,1.5299864,-.8451503, .3508961$, $-1.9093353,3.8264174,-.3860268,-.0060332,-.7141670,4.4447575$, $.0602218,-.1661942,-.9666144,1.2877476,-1.0778103,-1.2065677$,

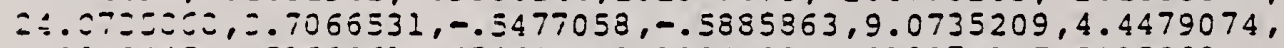
$-.0220445,-.5159251, .4546422,1.1824400,-.3183741,7.5035233$, -i0.5666030,3.6479535,-.2937472,2.6943143, $-4.3488745,4.4068680$, $-.2091134,-.1049981,-.0876423,1.4785960,1.3250118,-2.0488981$, $2.7983145,3.6850867, .1454941,-1.2364115,1.2334575,4.3319368$, $-.2962525,-. .842132, .0948841,1.7683451, .8524356, .4803464$, $-1.4080201,3.6504235,-.2636370,-.1215563,-.5012857,4.2642107 .$, $-.3598665,-.1258039, .0368399,1.9513209, .8717107,-.3864106$, $.2406320,3.5868773,-.3768426,-.4301406, .2054004,4.1853895$, $-.4068416,-.1031259, .0535461,2.1160002, .7435275,-.2382817$, $-.1725376,3.4932151,-.5274224,-.3036997, .0307898,4.0980282$, $-.4423998,-.0701638, .0610086,2.2570443, .6239388,-.3445240$, $-.0589758,3.3724694,-.6481685,-.2847460, .1015837,3.9500406$, $-.4680645,-.0150213, .0633914,2.4121404, .4002809,-.3978290$, $-.0380688,3.1541219,-.7920565,-.1928395, .1134978,3.8193913$, $-.4598536, .0422747, .0517269,2.4955854, .1501968,-.4322374$, $.7220555 E-04,2.9010892,-.8773478,-.0902549, .1058269,3.7272587$, $-.4359705, .0741170, .0598137,2.5082064,-.0271795,-.4321930$, $.0053276,2.7181764,-.9010199,-.0251096, .1241773,3.6414375$,

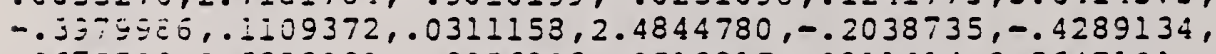
$.0675528,2.5333080,-.8956393, .0513317, .0911214,3.5647101$, $-.3485407, .1300916, .1036357,2.4251685,-.3713621,-.3873290$, 


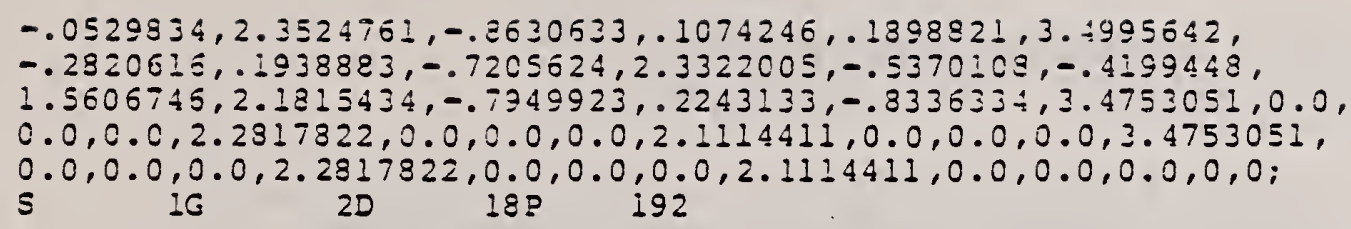


THIS IS A BENCKMARK OF A UNIECRM BSZLINE THAT IS OF DEGREE 3. POINTS WERE EXPLICITLY ENTERED AS EOLLOWS; $X 2 Y 5, X 6 Y 10, X 10 Y 6, X 14 Y 10, X 18 Y 6$

IH, , 1H; , 9HSYSTEM 1 , 25HEMC. BENCHMARK. 3SPLINE. PUT, 45 HCOMPUTERVISION. CADDSG 4 REV.2.00 GRAPHIC SYSTEM, 14HIGES REV 01.00,16,08,24,08,56,9 USYSTEM \#2, G $156.000,1,4 \mathrm{HINCH}, \ldots, 13 \mathrm{H} 810724.092702, \ldots 18 \mathrm{HINSTAVIEN}$ OPERATOR, 14 HCOMPUTERVC ISION

124

124

112

$$
1 \quad 3
$$

124,

112

$\begin{array}{cc}4 & 1 \\ 1.000000, & 0.000000, \\ 0.000000 ; & 1.000000 ; \\ 0.000000, & 0.000000, \\ 5 & 00000000,13\end{array}$

0.000000

$112,3,1,2,5, .0000000,1.000000,2.000000,3.000000,4.000000$

$5.000000,2.000000,3.980861,-0.2384186 \Sigma-06, .01913905,6.000000$,

$6.889953, .0000000,-2.889952, .0000000, .0000000, .0000000, .0000000$, $6.000000,4.038278, .05741692,-.09569439,10.00000,-1.779904$,

$-8.669857,6.449761, .0000000, .0000000, .0000000, .0000000,10.00000$, $3.866028,-.2296662, .3636374,6.000000, .2296648,10.67943$, $-6.909091, .0000000, .0000000, .0000000, .0000000,14.00000,4.497608$, $.8612461,-1.358853,10.00000, .8612442,-10.04785,5.186603$, $.0000000, .0000000, .0000000, .0000000,18.00000,2.143540,-3.215314$, $1.071773,6.000000,-3.574642,5.511961,-1.837319, .0000000$, $.0000000, .0000000, .0000000,18.00000,-1.071768, .00001120567$, $6.430639,6.000000,1.337322, .000005722046,-11.02392, .0000000$, $.0000000, .0000000, .0000000$;

S $4 \mathrm{G}^{\circ}$

$4 \mathrm{D}$

$4 P$

16

000000

000000

0.000000 0.000000 0.000000 


\subsubsection{Point}

Case 1 (Figure 3.3.7-1) tests the following:

- points derined in 3-D

- points with large absolute coordinate values

(-224355 to 200122)

Points are also tested in level Case 1 (Section 3.3.20). 


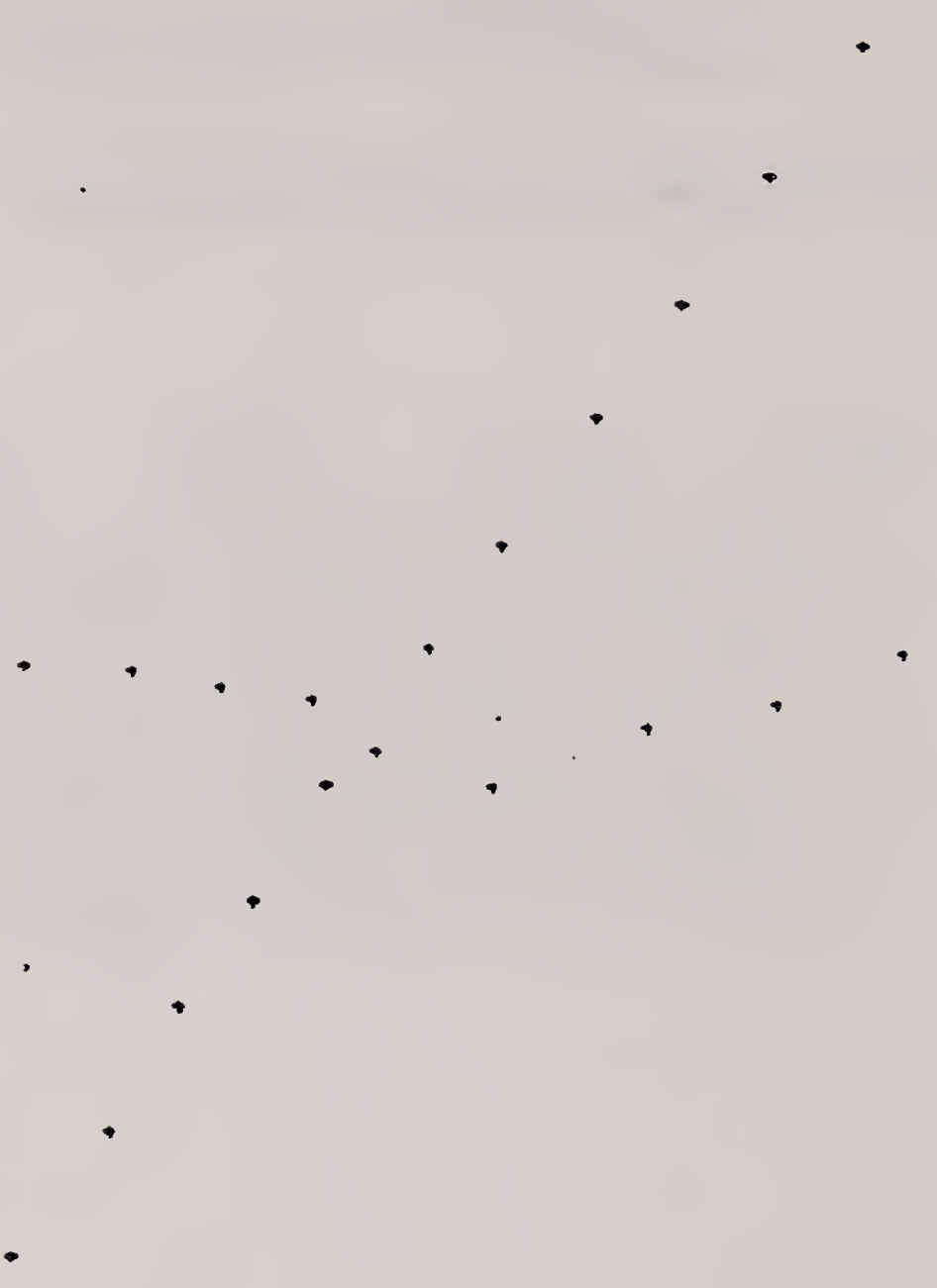

Figure 3.3.7-1 Point Case 1 (actual part) Case 1: Rotation $\left(0^{\circ}, 0^{\circ}, 0^{\circ}\right)$ 
POINT TEST CASE 1

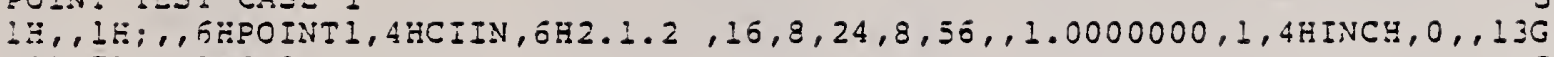

:810714.085049, , , i

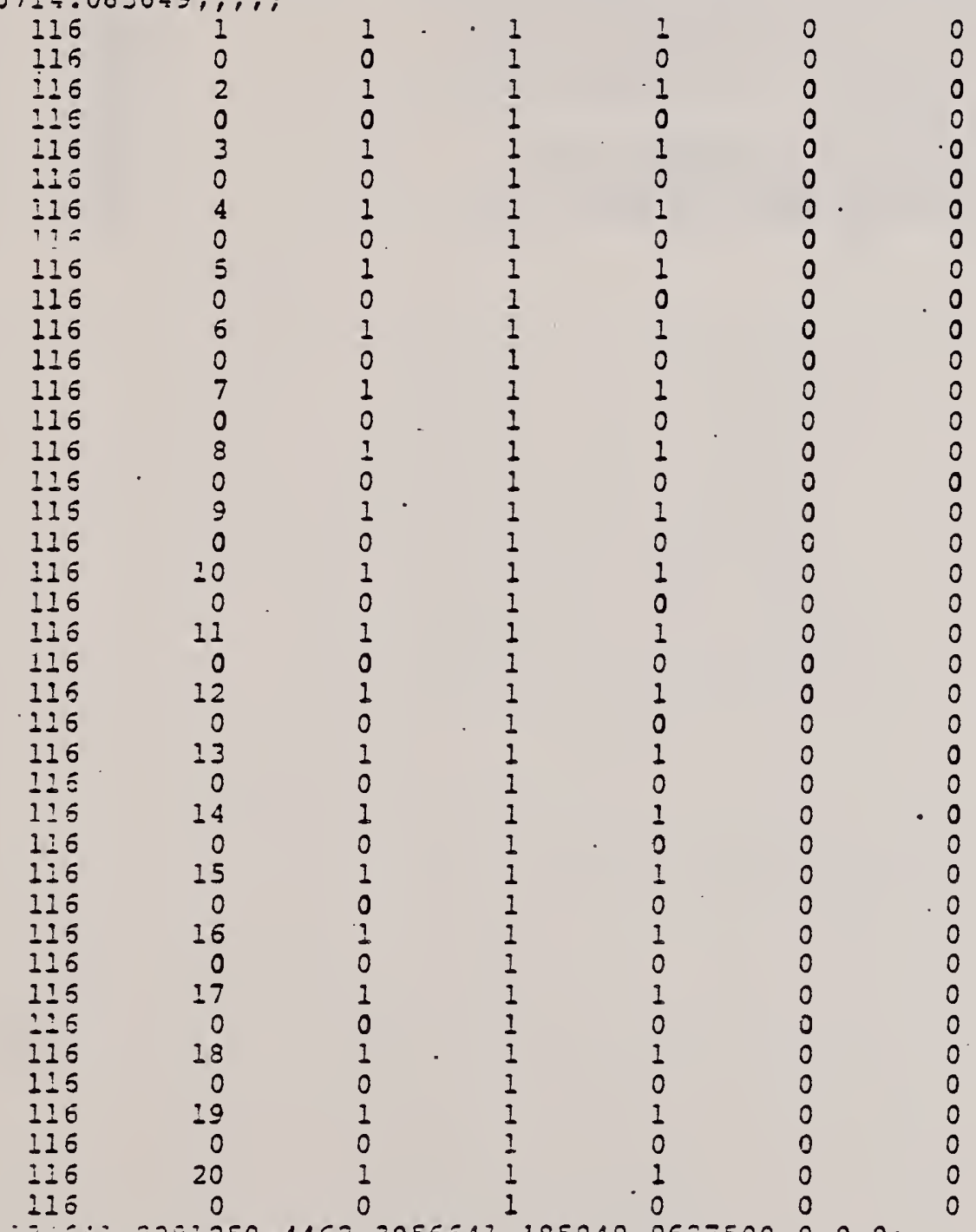

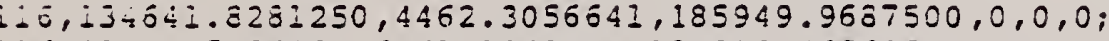

$126,98410.5156250,6261.1948242,132912.2656250,0,0,0$;

$116,59033.7734375,11927.7655313,85700.7109375,0,0,0$;

$116,38351.3945313,16344.7177734,37892.3398438,0,0,0$ :

$115,1740=2656250,33561.1757813,-3867.2158203,0,0,0$ :

II $==20-29.4380469,46052.3320313,-75366.8515025,0,0,0$;

$116,-72301.0000000,26884.7089844,-129133.7500000,0,0,0$;

$115,-114734.0781250,19728.5800781,-181987.0468750,0,0,0$;

$1: 0,-1 \equiv 5157.9218750,3472.6152344,-224355.6718750,0,0,0$;

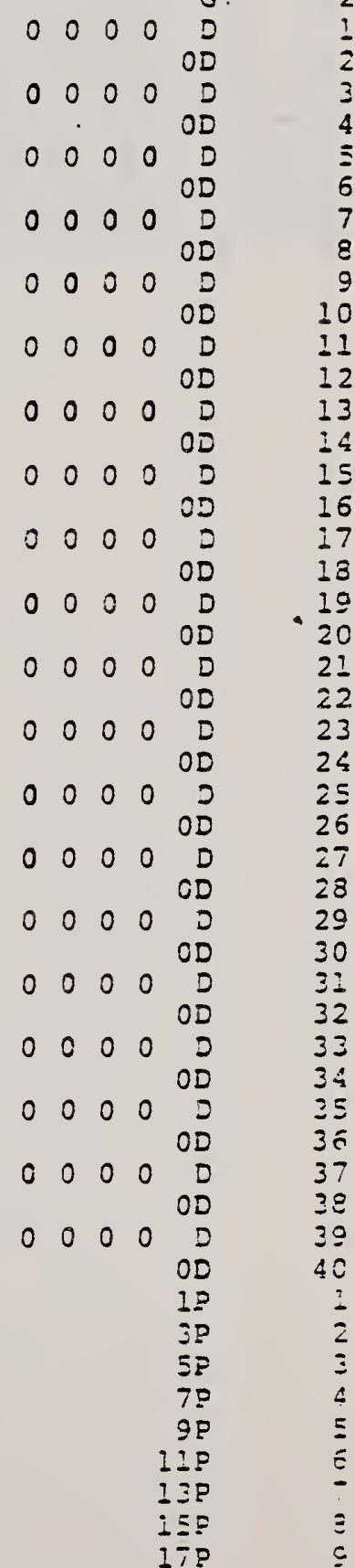


$116,-144753.6250000,-199308.9218750,-5417.7568359,0,0,0$; $116,-113754.3437500,-154860.2343750,-6013.4013555,0,0,0$; $116,-84704.5156250,-112122.7890625,-7667.4790039,0,0,0$; $116,-55980.5898438,-75165.0859375,-4003.4172353,0,0,0$; $116,140843.5781250,200122.8437500,-939.9491577,0,0,0$; $116,-24318.9882813,-33518.8710938,-873.3706055,0,0,0$; $115,107223.5312500,158071.0150250,-6434.053222 .7,0,0,0$; $116,-.0264881,-.4046917,0.0,0,0,0$;

$116,34434.4960938,44583.8359375,4113.8930664,0,0,0$; $116,58915.1171875,82457.9687500,850.5867310,0,0,0$; $116,84048.5468750,116876.8046875,1985.7882080,0,0,0$; 
3.3.8 Transformation Matrix

Transformation matrices are tested in most of the other cases. A wide range of values are included in those cases. 



\subsubsection{Angular Dimension}

The angular dimensions contain subentities which include leaders, witness lines, vertex points, and general notes.

The following conventions were followed:

- general note sub-entities to be single string with no box rotation

- $\quad$ arrows limited to one or two segments

- Case 1 (Figures 3.3.9-1 through 3.3.9-4) tests the following:

- text in, arrows in configuration

- text in, arrows out configuration

- $\quad$ acute and obtuse angles

- $\quad$ no witness lines suppressed

- $\quad$ first witness line suppressed

- $\quad$ second witness line suppressed

- both witness lines suppressed

- $\quad$ Case 2

- This is identical to Case 1 except non-model space with rotation $\left(-6.07^{\circ},-67.48^{\circ}, 92.37^{\circ}\right)$ is used per section 3.0

- Case 3 (Figures 3.3.9-5 and 3.3.9-6) tests the following:

- different witness line gaps

- very small angles

- nearly $360^{\circ}$ angles

- nearly $180^{\circ}$ angles

- Case 4

- This is identical to Case 3 except non-mocie! space with rotation $\left(11^{\circ}, 22^{\circ}, 33^{\circ}\right)$ is used per section 3.0 


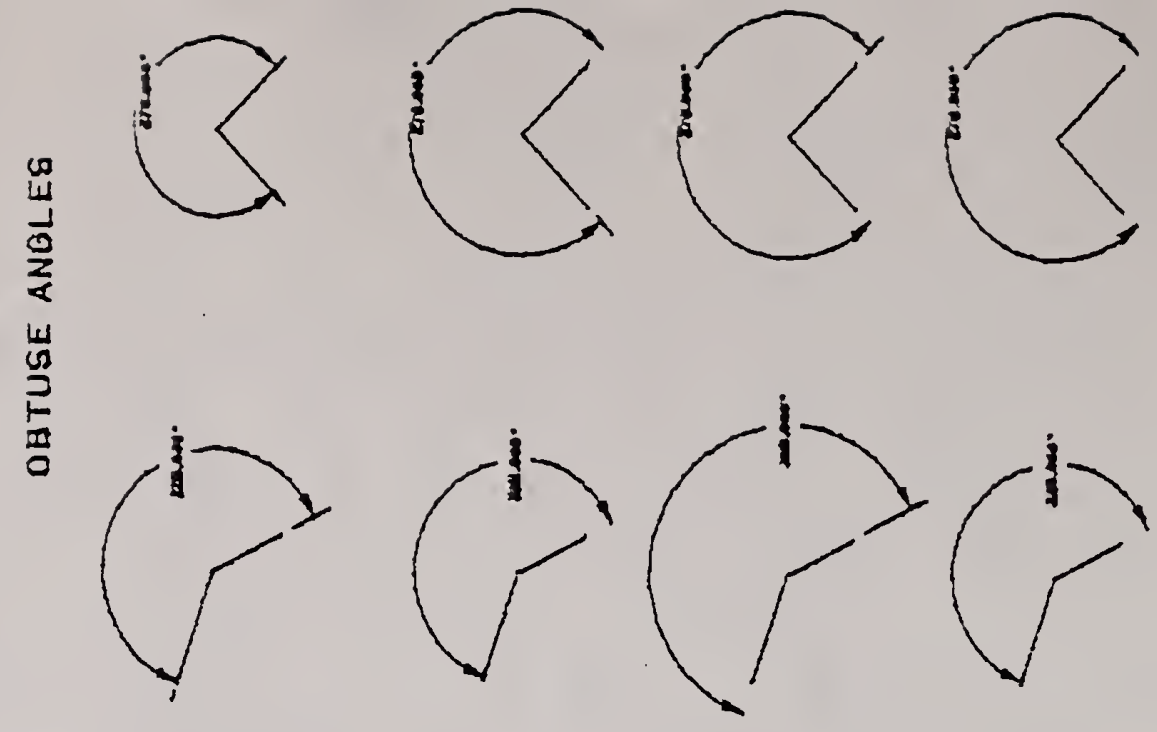

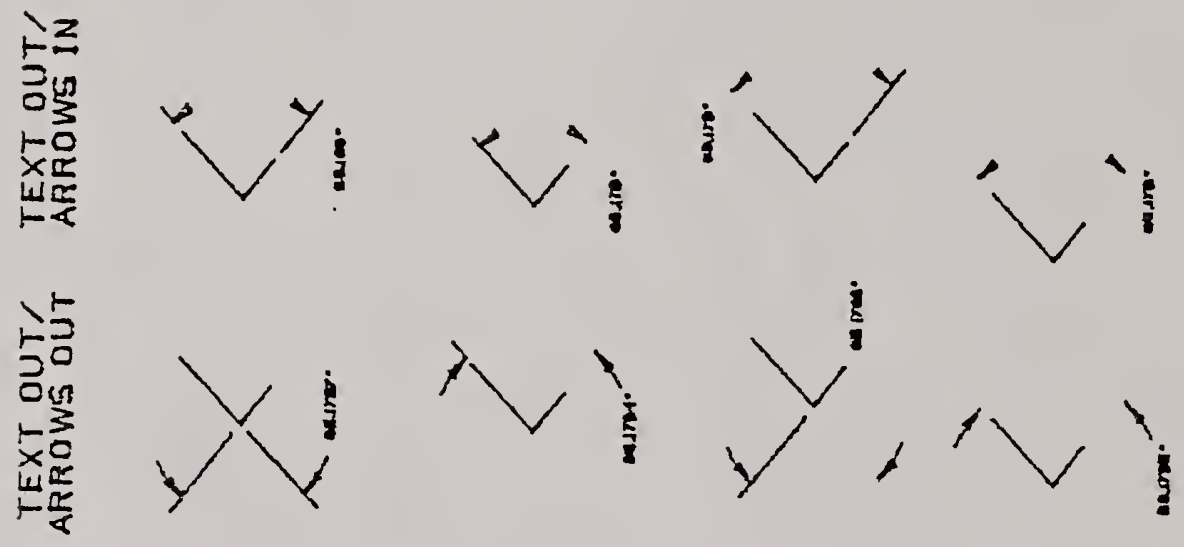

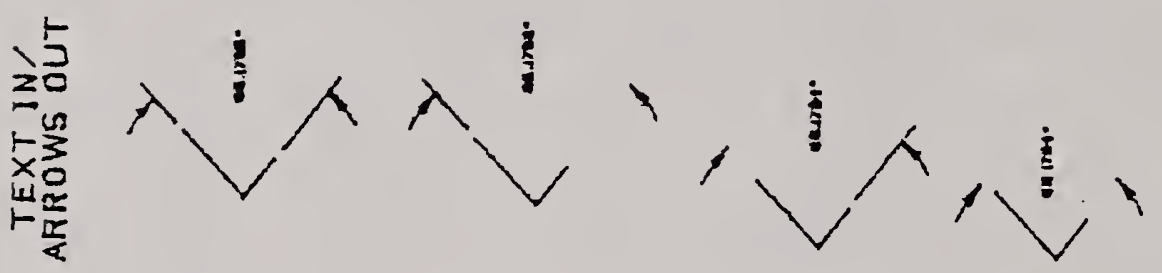<smiles>[C]1CCC1</smiles><smiles>C1CC1</smiles><smiles></smiles>

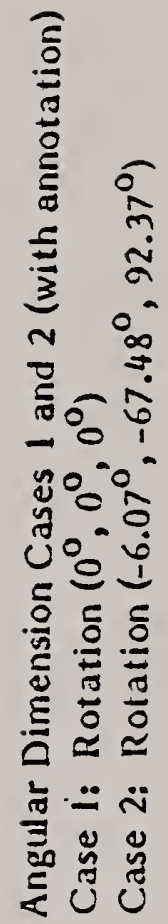
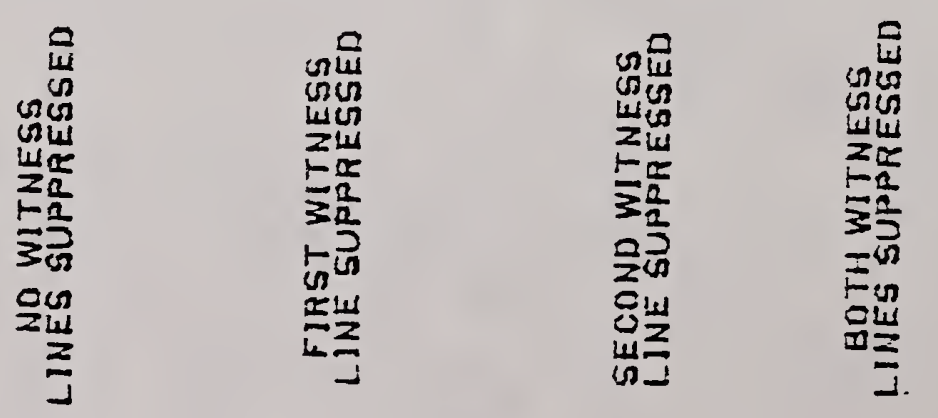

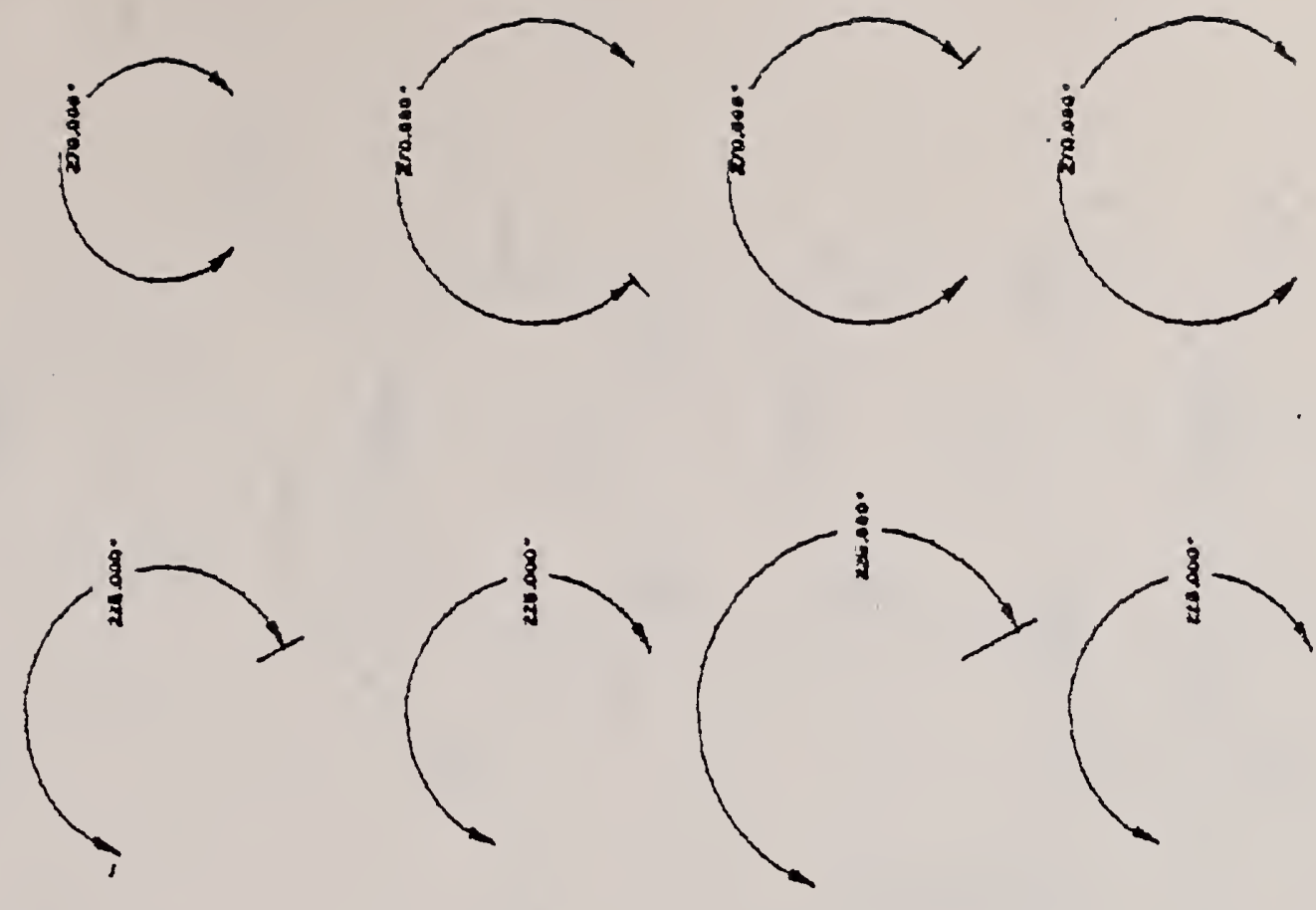

$\checkmark \quad Y_{\frac{j}{j}} \quad \Delta$

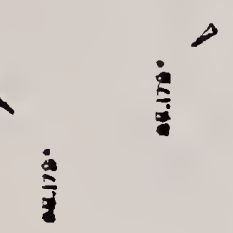

y

$y$

$>$

4

$\lambda \wedge \lambda$

,

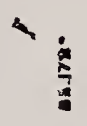



$<7$<smiles>CC[C](C)C</smiles><smiles>CC[C](C)CC</smiles> 


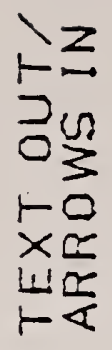
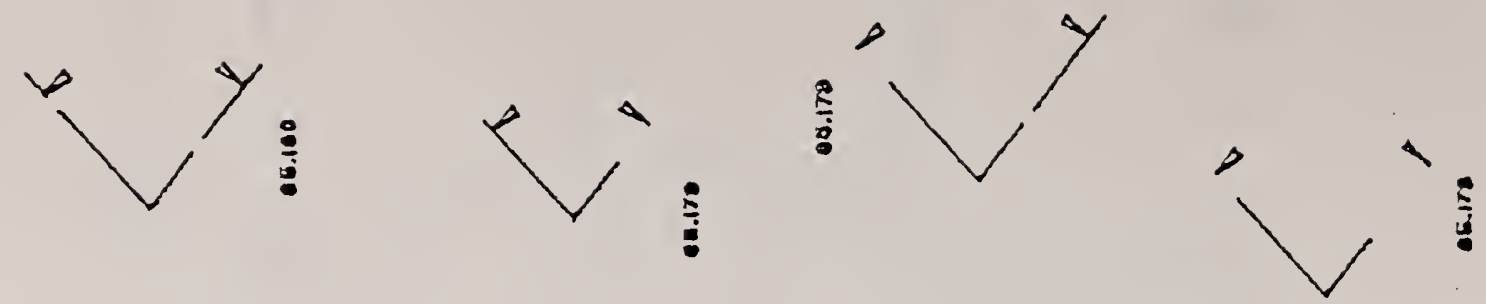

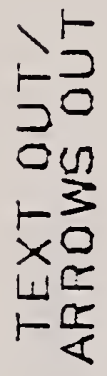
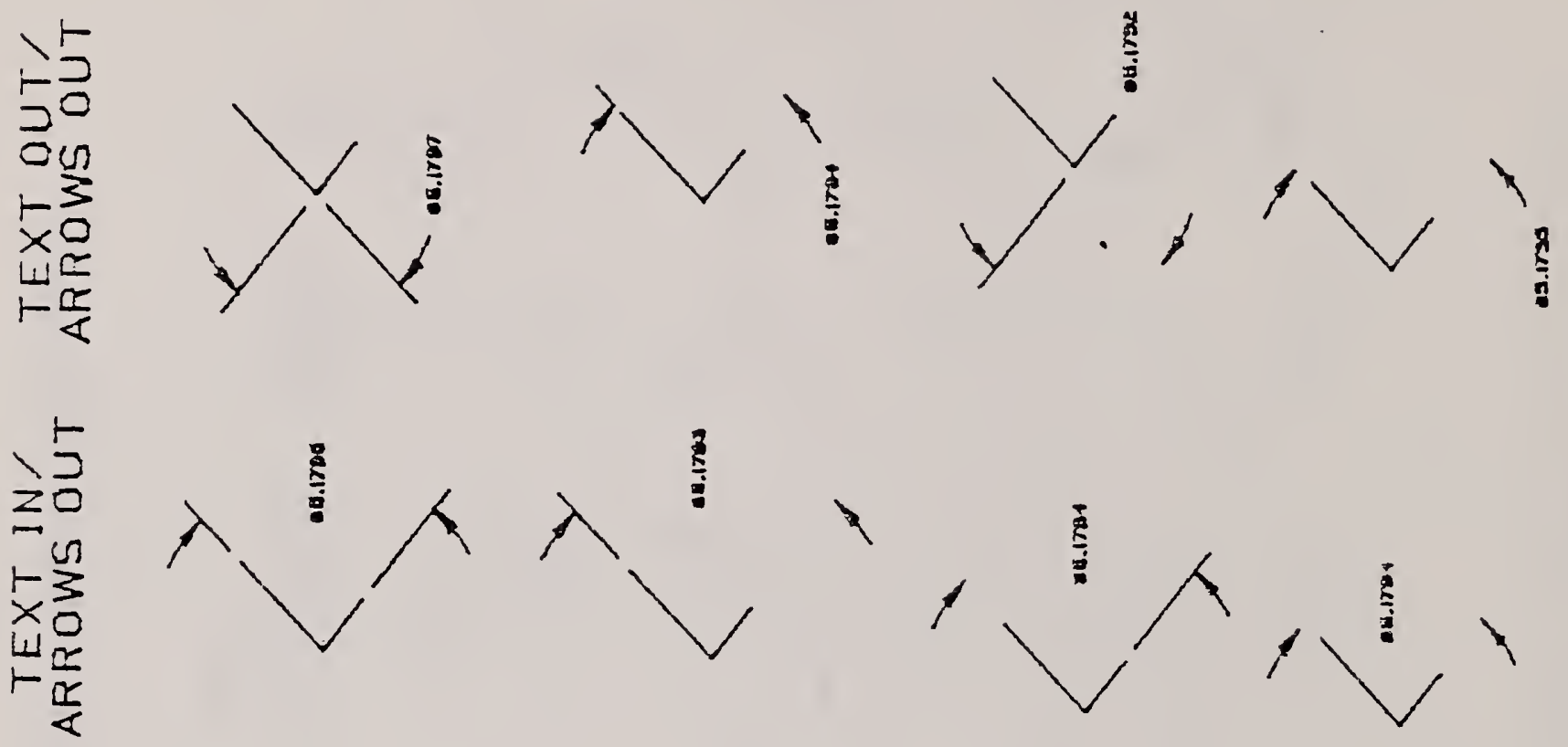

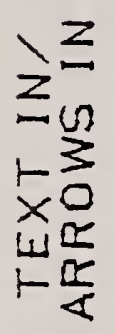
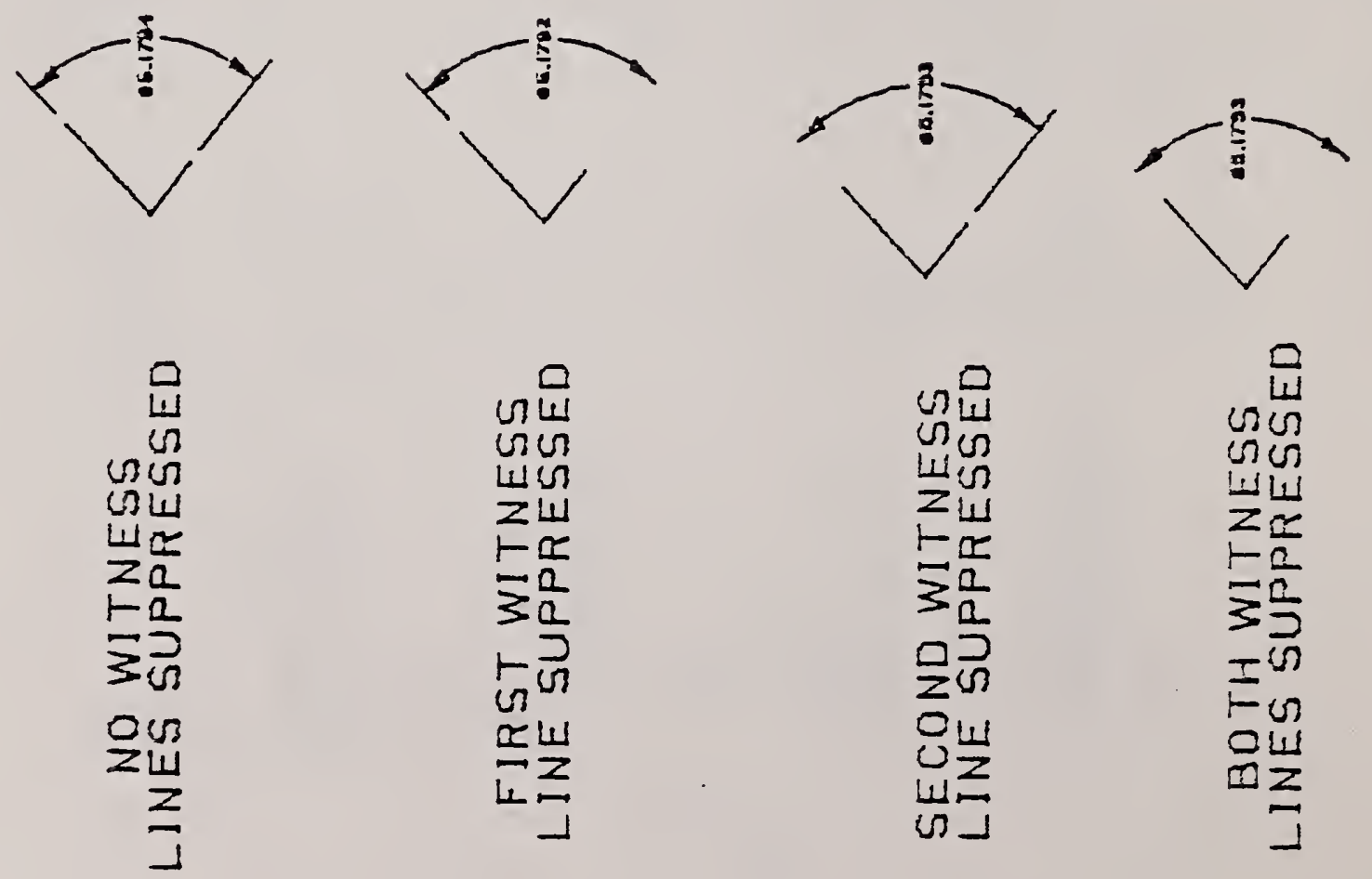

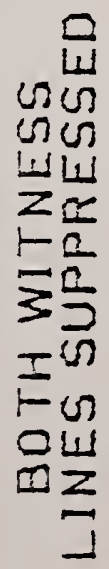



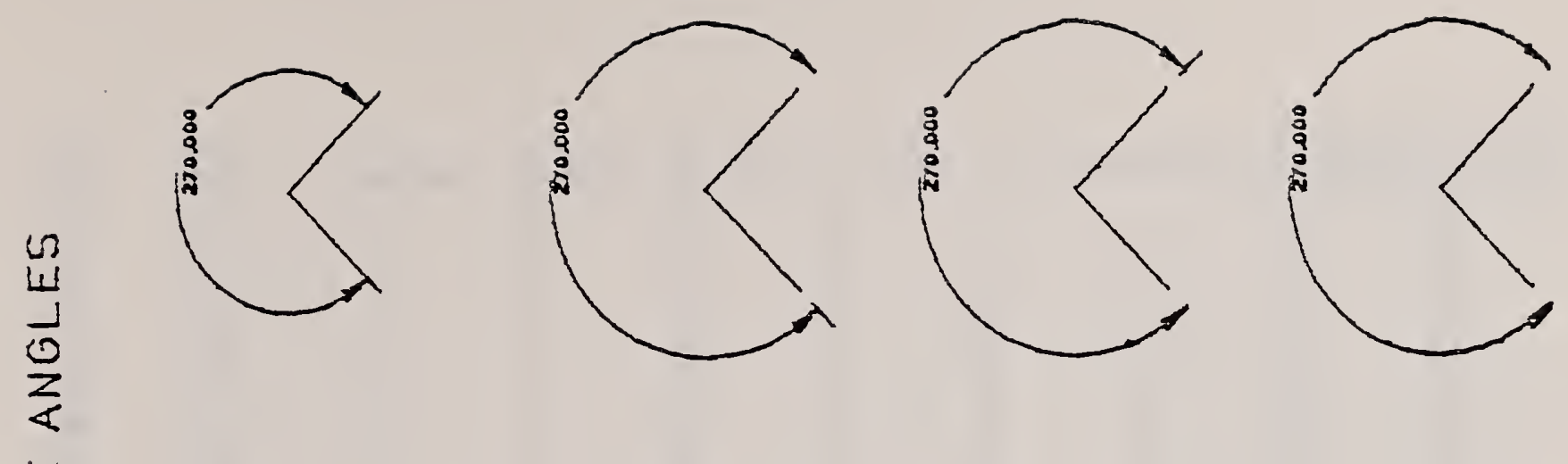

耑
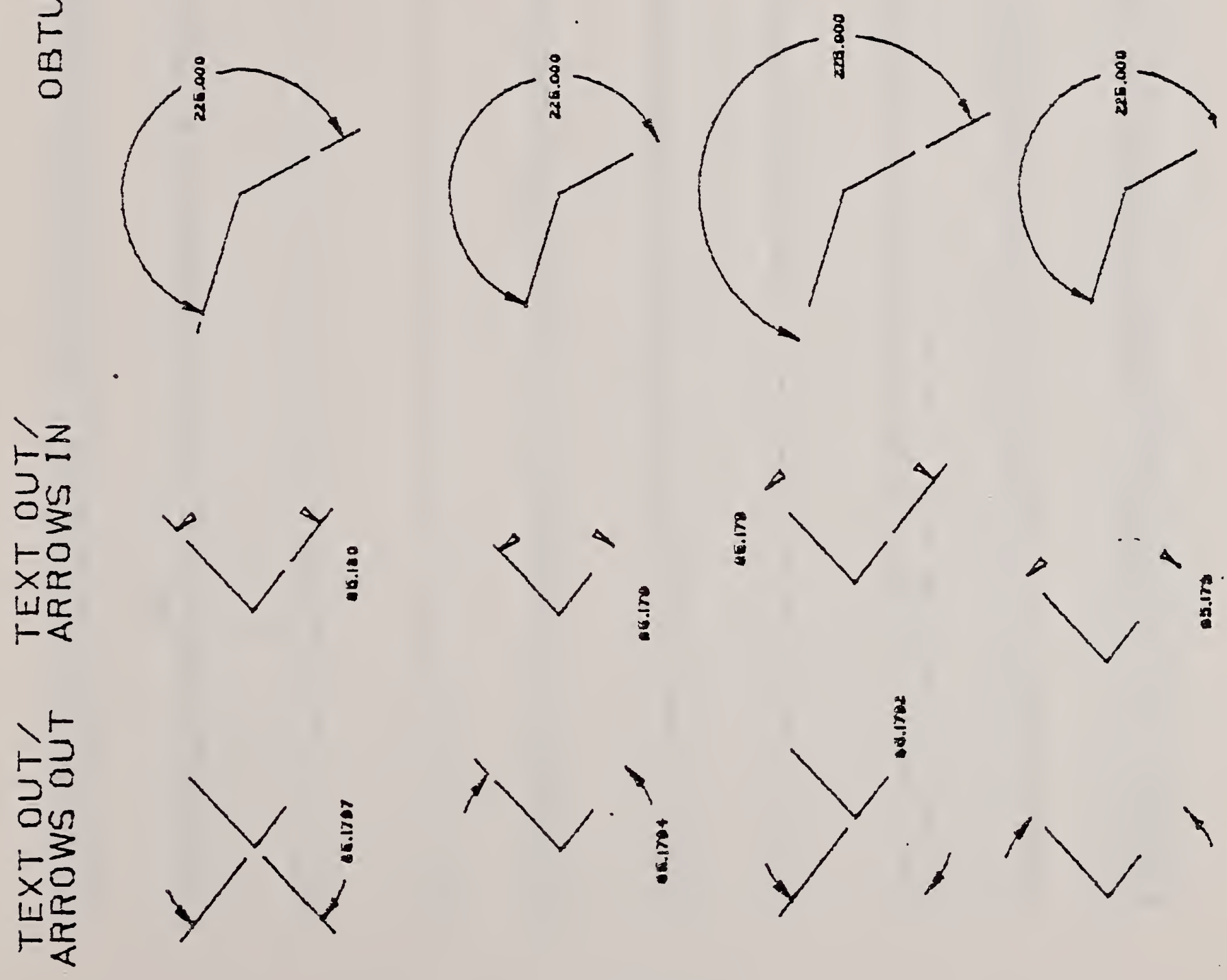

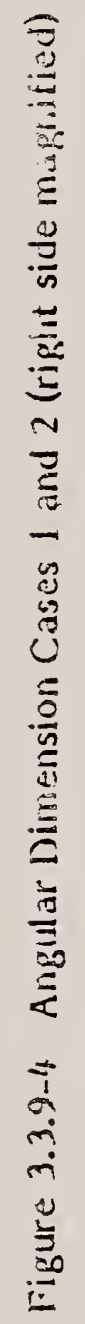




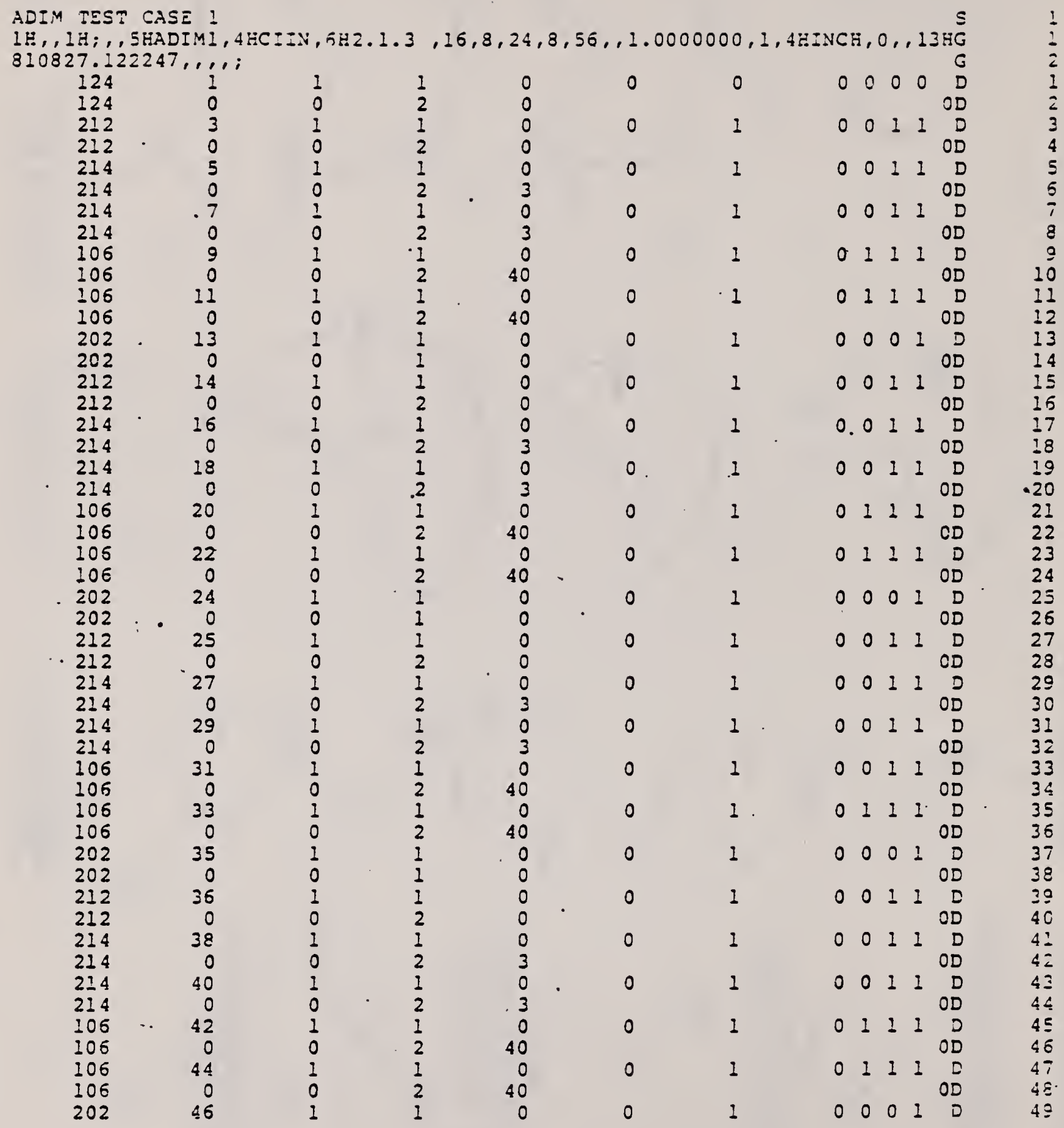




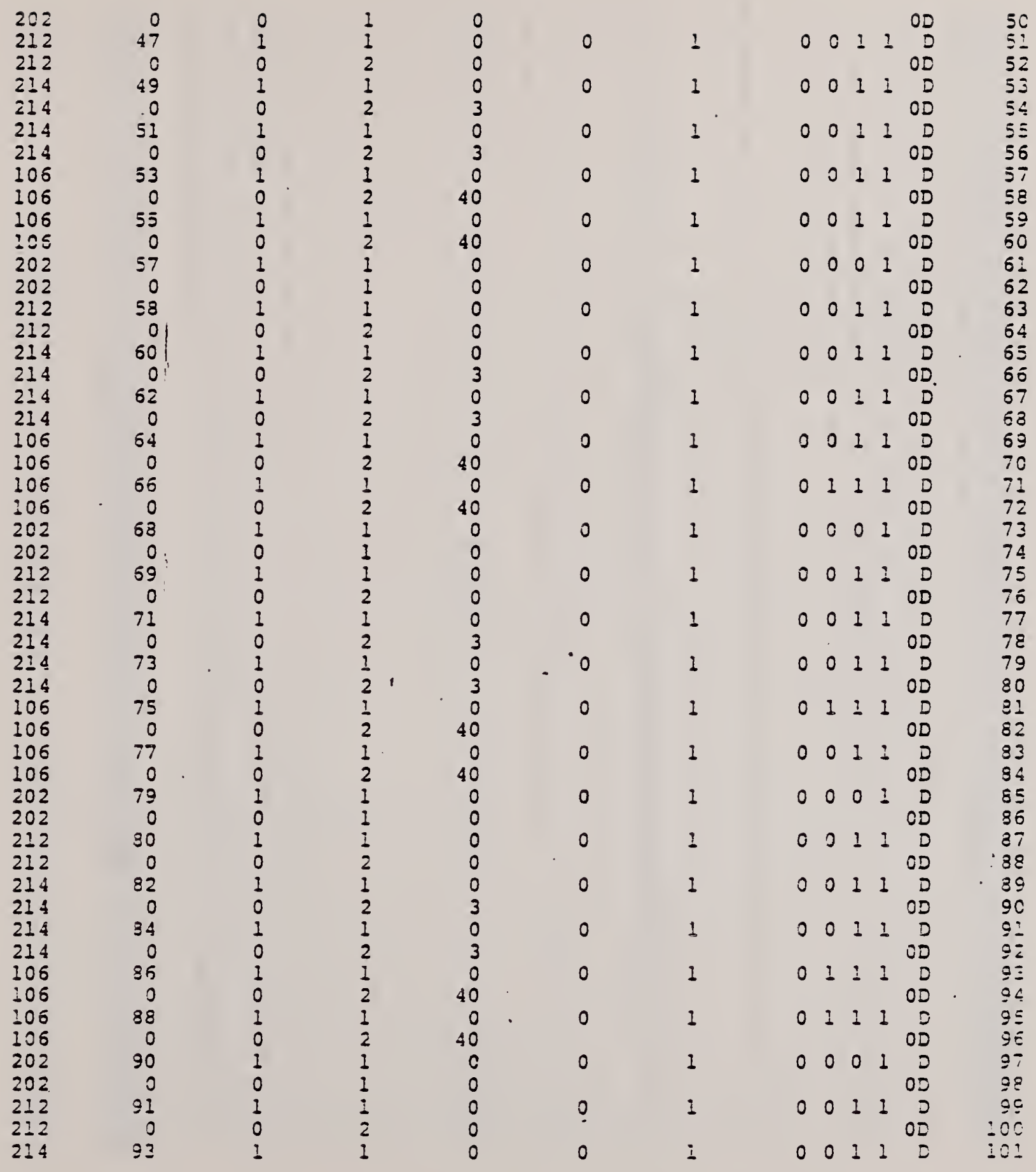




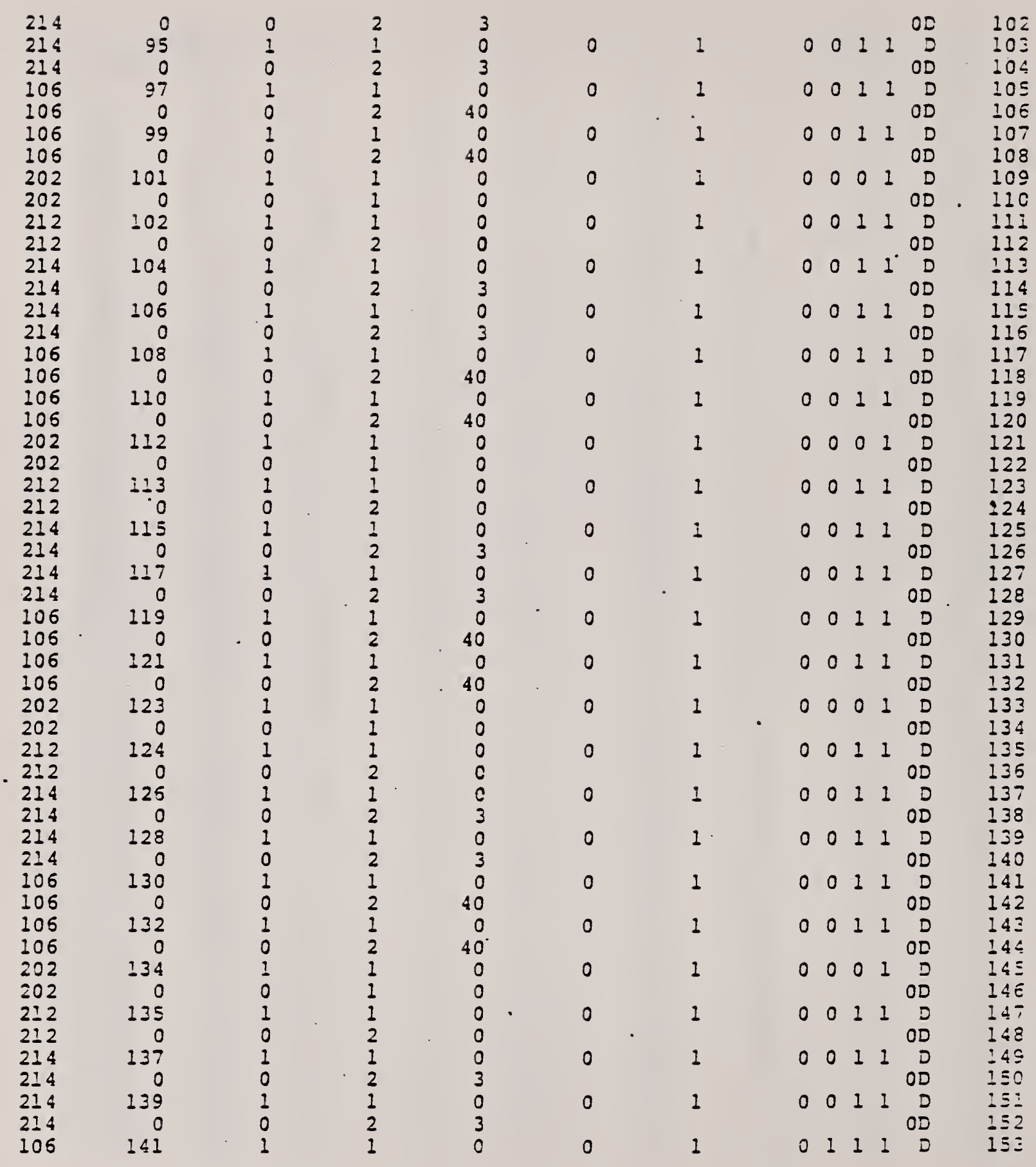




\begin{tabular}{|c|c|c|c|c|c|c|c|c|c|c|c|c|}
\hline $\begin{array}{l}106 \\
106\end{array}$ & $\begin{array}{r}0 \\
143\end{array}$ & $\begin{array}{l}0 \\
1\end{array}$ & $\begin{array}{l}2 \\
1\end{array}$ & $\begin{array}{r}40 \\
0\end{array}$ & 0 & 1 & 0 & 0 & 1 & & $\begin{array}{l}O D \\
0\end{array}$ & $\begin{array}{l}150 \\
15\end{array}$ \\
\hline 106 & 0 & 0 & 2 & 40 & & & & & & & DD & 15ถ \\
\hline 202 & 145 & $\frac{1}{1}$ & $\frac{1}{1}$ & 0 & 0 & 1 & 0 & 0 & 0 & ? & D & $\begin{array}{l}155 \\
1=2\end{array}$ \\
\hline 202 & 0 & 0 & 1 & 0 & & & & & & & OD & $1 \equiv 2$ \\
\hline 212 & 146 & 1 & 1 & 0 & 0 & 1 & 0 & 0 & 1 & 1 & D & 159 \\
\hline $\begin{array}{l}212 \\
214\end{array}$ & $\begin{array}{r}0 \\
148\end{array}$ & $\begin{array}{l}0 \\
1\end{array}$ & $\begin{array}{l}2 \\
1\end{array}$ & $\begin{array}{l}0 \\
0\end{array}$ & 0 & 1 & 0 & 0 & 1 & 1 & $\begin{array}{c}O D \\
D\end{array}$ & $\begin{array}{l}160 \\
161\end{array}$ \\
\hline 214 & 0 & 0 & $\frac{1}{2}$ & 3 & 0 & 1 & 0 & 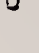 & 1 & & $O D$ & 162 \\
\hline 214 & $1 \leq 0$ & 1 & 1 & 0 & 0 & 1 & 0 & 0 & 1 & $?$ & D & 153 \\
\hline 214 & 0 & 0 & 2 & 3 & & & & & & & $O D$ & 164 \\
\hline 106 & 152 & 1 & 1 & 0 & 0 & 1 & 0 & 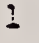 & 1 & 1 & $D$ & 165 \\
\hline 106 & 0 & 0 & 2 & 40 & & & & & & & $O D$ & 166 \\
\hline 106 & 254 & 1 & 1 & 0 & 0 & 1 & 0 & 0 & 1 & & $D$ & 167 \\
\hline 106 & 0 & 0 & 2 & 40 & & & & & & & DD & 158 \\
\hline 202 & 156 & 1 & 1 & 0 & 0 & 1 & 0 & 0 & 0 & 1 & $D$ & 169 \\
\hline 202 & 0 & 0 & 1 & 0 & & & & & & & $O D$ & 170 \\
\hline 212 & 157 & 1 & 1 & 0 & 0 & 1 & 0 & 0 & 1 & & $D$ & 171 \\
\hline 212 & 0 & 0 & 2 & 0 & & & & & & & OD & 172 \\
\hline 214 & $1 \leq 9$ & 1 & 1 & 0 & 0 & 1 & 0 & 0 & 1 & & 0 & 173 \\
\hline 214 & $?$ & 0 & 2 & 3 & & & & & & & OD & 174 \\
\hline 214 & 161 & 1 & 1 & 0 & 0 & 1 & 0 & 0 & 1 & & $D$ & 175 \\
\hline 214 & 0 & 0 & 2 & 3 & & & & & & & $C D$ & 175 \\
\hline 106 & 163 & 1 & 1 & 0 & 0 & 1 & 0 & 1 & 1 & 1 & $D$ & 177 \\
\hline 106 & $\stackrel{0}{0}$ & 0 & 2 & 40 & & & & & & & OD & 178 \\
\hline 106 & 165 & 2 & 1 & 0 & 0 & 1 & 0 & 0 & 1 & & $D$ & 179 \\
\hline 106 & 0 & 0 & 2 & 40 & & & & & & & OD & 180 \\
\hline $\begin{array}{l}202 \\
202\end{array}$ & $\begin{array}{r}167 \\
0\end{array}$ & $\frac{1}{0}$ & $\frac{1}{1}$ & $\begin{array}{l}0 \\
0\end{array}$ & 0 & 1 & 0 & 0 & 0 & 1 & D D & $\begin{array}{l}18: \\
182\end{array}$ \\
\hline 212 & 168 & 1 & $\vec{I}$ & 0 & 0 & 1 & 0 & 0 & 1 & 1 & 0 & 183 \\
\hline 212 & & 0 & 2 & 0 & & & & & & & DD & \\
\hline 214 & 170 & 1 & 1 & 0 & 0 & 1 & 0 & 0 & 1 & $?$ & $D$ & 185 \\
\hline 214 & 0 & 0 & 2 & 3 & & & & & & & $O D$ & 86 \\
\hline 214 & 172 & $I$ & 1 & 0 & 0 & 1 & 0 & 0 & 1 & 1 & $D$ & 187 \\
\hline 214 & 0 & 0 & 2 & 3 & & & & & & & $O D$ & 88 \\
\hline 106 & 174 & 1 & 1 & 0 & 0 & 2 & 0 & 1 & 1 & 1 & $D$ & 189 \\
\hline 106 & 0 & 0 & 2 & 40 & & & & & & & D & 190 \\
\hline 106 & 176 & 1 & 1 & 0 & 0 & 1 & 0 & 1 & 1 & 1 & $D$ & 191 \\
\hline 106 & 0 & 0 & 2 & 40 & & & & & & & $O D$ & 192 \\
\hline 202 & 178 & 1 & 1 & 0 & 0 & 1 & 0 & 0 & 0 & 3 & $D$ & 193 \\
\hline 202 & 0 & 0 & 1 & 0 & & & & & & & $O D$ & 194 \\
\hline 212 & 179 & 1 & 1 & 0 & 0 & 1 & 0 & 0 & 1 & 1 & $D$ & $19 \equiv$ \\
\hline $2: 2$ & 0 & 0 & 2 & 0 & & & & & & & $O D$ & 195 \\
\hline 214 & 181 & 1 & 1 & 0 & 0 & 1 & 0 & 0 & 1 & 1 & $D$ & 197 \\
\hline $2: 4$ & 0 & 0 & 2 & 3 & & & & & & & OD & 198 \\
\hline $2: 4$ & 183 & $!$ & 1 & 0 & 0 & 1 & 0 & 0 & 1 & 1 & $D$ & $19 \Omega$ \\
\hline 214 & 0 & 0 & 2 & 3 & & & & & & & $O D$ & 200 \\
\hline 106 & 185 & 1 & 1 & 0 & 0 & 1 & 0 & 0 & 1 & 1 & $D$ & 201 \\
\hline 106 & 0 & 0 & 2 & 40 & & & & & & & $O D$ & 202 \\
\hline 106 & 187 & 1 & 1 & 0 & 0 & 1 & 0 & 1 & 1 & 1 & $D$ & 203 \\
\hline 106 & 0 & 0 & 2 & 40 & & & & & & & $O D$ & $20 \div$ \\
\hline 202 & 189 & 1 & 1 & 0 & 0 & 1 & 0 & 0 & 0 & $i$ & $D$ & 05 \\
\hline
\end{tabular}




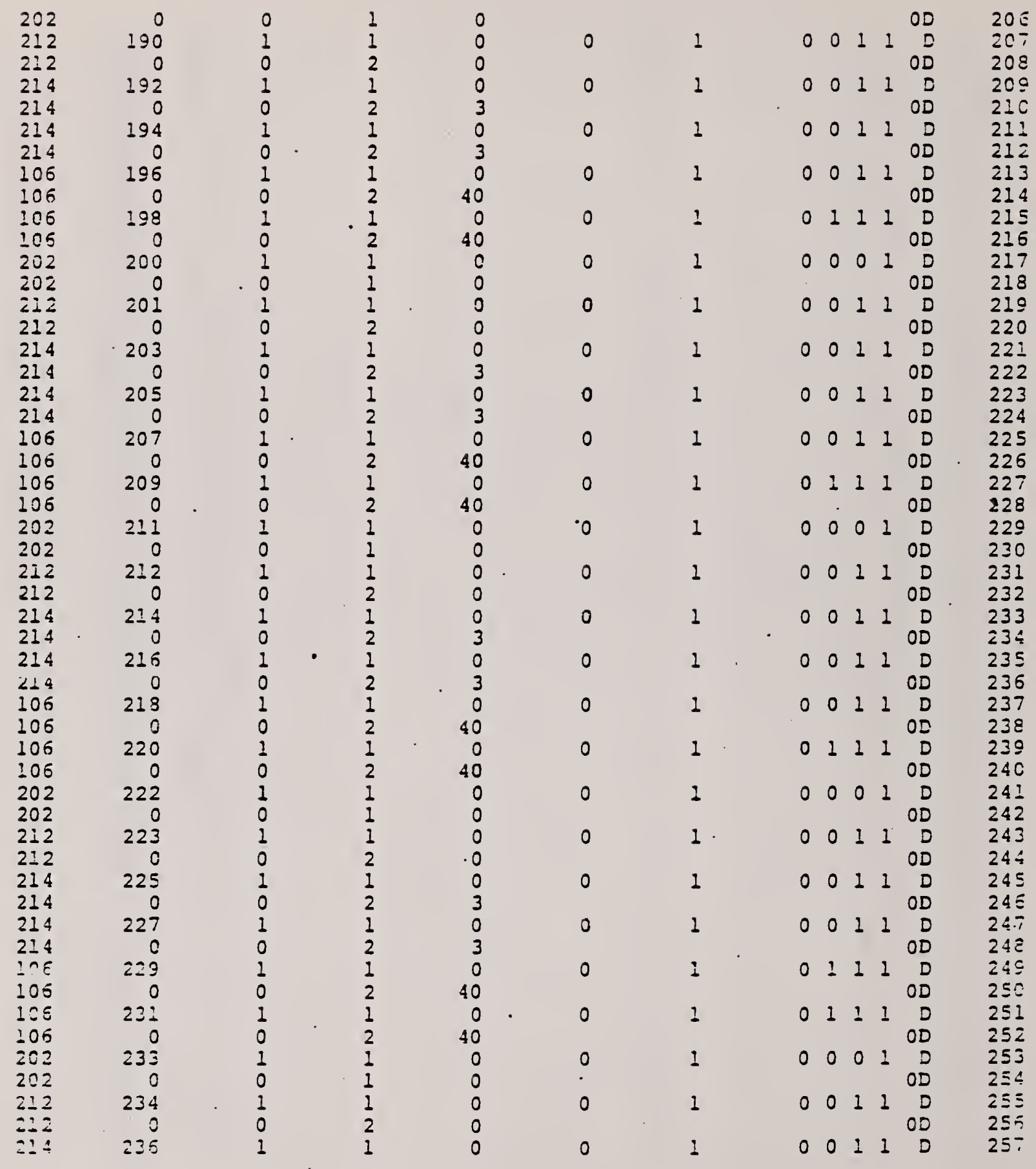




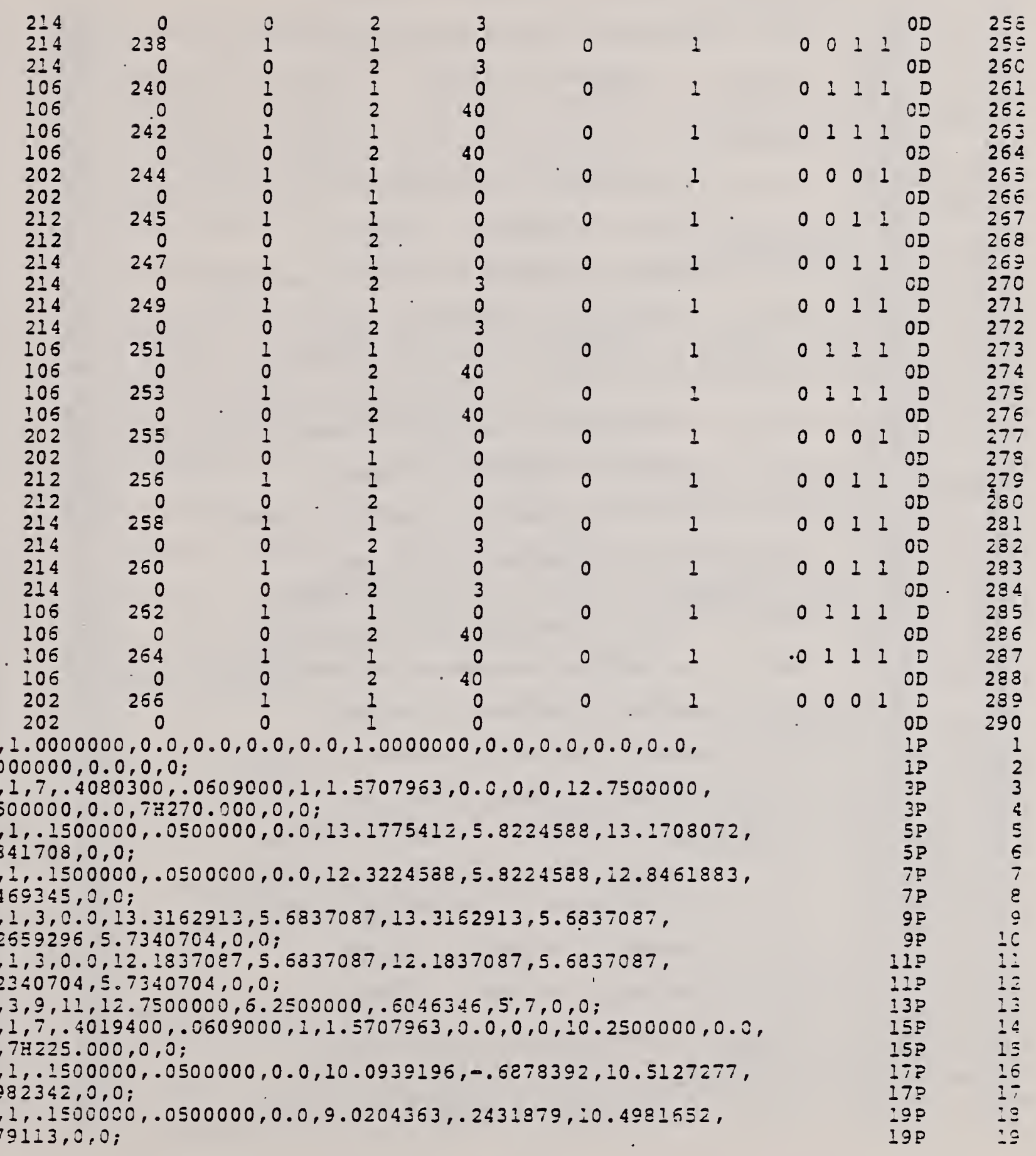

$3.9-11$ 
$105,1,3,0.0,10.0419263,-.5838525,10.0419263,-.5838525$,

$\div 0.1498213,-.7996426,0,0$;

$106,1,3,0.0,8.9110609, .2796464,8.9110609, .2796464,8.9018509$, $.28271 .64,0,0$;

$202,15,21,23,9.7500000,0.0, .7690277,17,19,0,0$;

$212,1,7, .4019400, .0609000,1,1.5707963,0.0,0,0,20.5000000$,

$2.0000000,0.0,7 \mathrm{iz} 225.000,0,0$;

$214,1, .1500000, .0500000,0.0,10.2056059,1.0887882,10.7639457$, $1.9010157,0,0$;

$214,1, .1500000, .0500000,0.0,8.7835139,2.3221620,10.7528776$,

$2.1792215,0,0$;

$106,1,3,0.0,10.0419263,1.4161475,10.0419263,1.4161475$,

$10.2515076, .9769848,0,0$;

$106,1,3,0.0,8.9110609,2.2796464,8.9110609,2.2796464,8.6649285$, $2.3616905,0,0$;

$202,27,33,35,9.7500000,2.0000000,1.0187658,29,31,0,0$;

$212,1,7, .4019400, .0609000,1,1.5707963,0.0,0,0,10.2500000$, $4.0000000,0.0,714225.000,0,0$;

$214,1, .1500000, .0500000,0.0,10.0939196,3.3121608,10.5127277$,

$3.9017660,0 ., 0$;

$214,1, .1500000, .0500000,0.0,9.0204363,4.2431879,10.4981651$, $4.1779116,0,0$ :

$106,1,3,0.0,10.0419263,3.4161475,10.0419263,3.4161475$,

$10.1498213,3.2003574,0,0$;

$106,1,3,0.0,8.9110609,4.2796464,8.9110609,4.2796454,8.9018509$, $4.2827164,0,0$;

$202,39,45,47,9.7500000,4.0000000, .7690277,41,43,0,0$;

$212,1,7, .4019400, .0609000,1,1.5707963,0.0,0,0,10.2500000$,

$6.5000000,0.0,7 \mathrm{H} 225.000,0,0$;

$214,1, .1500000, .0500000,0.0,10.1171928,5.5156143,10.5564698$ ，

$6.4041407,0,0$;

$214,1, .1500000, .0500000,0.0,8.9710664,5.5096446,10.4555812$,

$6.6582056,0,0$

$106,1,3,0.0,10.0419263,5.6661475,10.0419263,5.6061475$,

$10.1730945,5.4038109,0,0$;

$106,1,3,0.0,8.911060 .9,5.5296464,8.9110609,6.5296464,8.8524810$, $6.5491730,0,0$ :

$202,51,57,59,9.7500000,6.2500000, .3210682,53,55,0,0$;

$212,1,7, .4080300, .0609000,1,1.5707963,0.0,0,0,12.7500000$,

$2.7500000,0.0,7 \mathrm{E} 270.000,0,0$;

$214,1, .1500000, .0500000,0.0,13.3409027,1.4090973,13.2171124$, $2.6929199,0,0$;

$214,1, .1500000, .0500000,0.0,12.1590973,1.4090973,12.8062794$, $2.8337653,0,0$;

$106,1,3,0.0,13.3162913,1.4337087,13.3162913,1.4337087$,

$13.4292910,1.3207090,0,0$;

$106,1,3,0.0,12.1837087,1.4337087,12.1837087,1.4337037$,

$12.0707090,1.3207090,0,0$;

$202,53,69,71,12.7500000,2.0000000, .8355626,65,67,0,0$;

$212,1,7, .4080300, .0609000,1,1.5707963,0.0,0,0,12.7500000$,

$4.7500000,0.0,7 \mathrm{H} 270.000,0,0$;

$214,1, .1500000, .0500000,0.0,13.3409028,3.4090972,13.2171053$,

$\begin{array}{ll}21 P & 2 C \\ 21 P & 21 \\ 23 P & 22 \\ 23 P & 23 \\ 25 P & 21 \\ 27 P & 25 \\ 27 P & 26 \\ 29 P & 27 \\ 29 P & 28 \\ 31 P & 29 \\ 31 P & 30 \\ 33 P & 31 \\ 33 P & 32 \\ 35 P & 33 \\ 35 P & 34 \\ 37 P & 35 \\ 39 P & 36 \\ 39 P & 37 \\ 41 P & 38 \\ 41 P & 39 \\ 43 P & 40 \\ 43 P & 41 \\ 45 P & 42 \\ 45 P & 43 \\ 47 P & 44 \\ 47 P & 45 \\ 49 P & 46 \\ 51 P & 47 \\ 51 P & 48 \\ 53 P & 49 \\ 53 P & 50 \\ 55 P & 51 \\ 55 P & 52 \\ 57 P & 53 \\ 57 P & 54 \\ 59 P & 55 \\ 59 P & 55 \\ 61 P & 57 \\ 63 P & 58 \\ 63 P & 59 \\ 65 P & 60 \\ 65 P & 69 \\ 67 P & 62 \\ 67 P & 53 \\ 69 P & 54 \\ 69 P & 55 \\ 71 P & 56 \\ 71 P & 67 \\ 73 P & 68 \\ 75 P & 65 \\ 75 P & 70 \\ 77 P & 79 \\ & \end{array}$


$4.6929249,0,0$;

$214, \therefore, .: 500000, .0500000,0.0,12.1590972,3.4090972,12.8062877$, $4.83375 \div 3,0,0$

$106,1,3,0.0,13.3162913,3.4337087,13.3162913,3.4337087$,

$13.4292912,3.3207088,0,0$;

$106,1,3,0.0,12.1837087,3.4337087,12.1837087,3.4337087$,

$12.0707088,3.3207088,0,0$;

$202,75,81,83,12.7500000,4.0000000, .8356628,77,79,0,0$;

$212,1,7, .4080300, .0609000,1,1.5707963,0.0,0,0,12.7500000$,

$.7500000,0.0,75270.000,0,0$;

$214, ?, .1500000, .0500000,0.0,13.3409027,-.5909027,13.2171124$,

$.6929109,0,0$;

$214,1, .1500000, .0500000,0.0,12.1590973,-.5909027,12.3062794$, $.8337653,0,0$;

$106,1,3,0.0,13.3152913,-.5662913,13.3152913,-.5662913$,

$13.4292910,-.6792910,0,0$;

$105,1,3,0.0,12.1837087,-.5662913,12.1837087,-.5662913$,

$12.0707090,-.6792910,0,0$;

$202,87,93,95,12.7500000,0.0, .8356626,89,91,0,0$;

$212,1,7, .4019400, .0609000,1,1.5707963,0.0,0,0,1.3335195$,

$6.0924616,0.0,71885.1794,0,0$;

$214,1, .1 \equiv 00000, .0500000,0.0,1.41296 \equiv 2,5.5212946,1.5220727$,

$6.0315438,0,0$ :

$214,1, .1500000, .0500000,0.0,1.3618900,6.7547125,1.6174298$,

$5.2142524,0,0$;

$100,1,3,0.0,1.0592672,5.8199757,1.0592672,5.8199757,1.5084637$, $5.4405467,0,0 ;$

$106,1,3,0.0,1.2459306,6.6187529,1.2459306,6.6187529,1.4502783$, $6.8231009,0,0$;

$202,09,105,107, .7273991,6.1002216, .8973057,101,103,0,0$;

$212,1,7, .3958500, .0509000,1,1.5707963,0.0,0,0,3.7858014$,

$6.1401320,0.0,7185.1795,0,0$;

$214,1, .1500000, .0500000,0.0,3.8469553,5.5143649,3.7152957$,

$5.3865646,0,0$;

$214,1, .1500000, .0500000,0.0,3.7924364,6.3086880,3.6515481$, $6.9249570,0,0$;

$106,1,3.0 .0,3.4475477,5.8515462,3.4475477,5.8516462,3.9424585$, $5.4337157,0,0$;

$105,1,3,0.0,3.6342111,6.6504234,3.6342111,6.6504234,3.8808649$, $6.8970763,0,0$;

$202,111,1: 7,119,3.1256802,6.1318932, .9571335,123,115,0,0$;

$212,1,7, .395 \varepsilon \equiv 00, .0609000, ?, 1.5707963,0.0,0,0,5.4941936$,

$5.5085507,0.0,7585.1797,0,0$;

$=: . .500000, .0500000,0.0,4.9675483,6.6129165,5.1033343$ ，

$6.73515 i 7,0,0$;

$z: \dot{z}, 2, .5500000, .0500000,0.0,5.0057302,5.7057691,5.2932935$,

$5.5388007,5.4332935,5.5388007,0,0$;

$105,1,3,0.0,5.4084452,6.2405977,5.4084452,6.2405 \Xi 77,4.8720451$, $5.6025540,0,0$;

$106,1,3,0.0,5.1137822,6.1138211,5.4137822,6.1138211,4.9173418$, $\overline{0} 0 \div \div 30 \div, 0,0$;

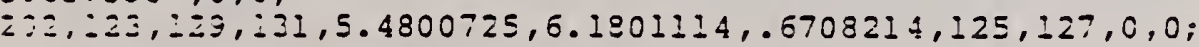

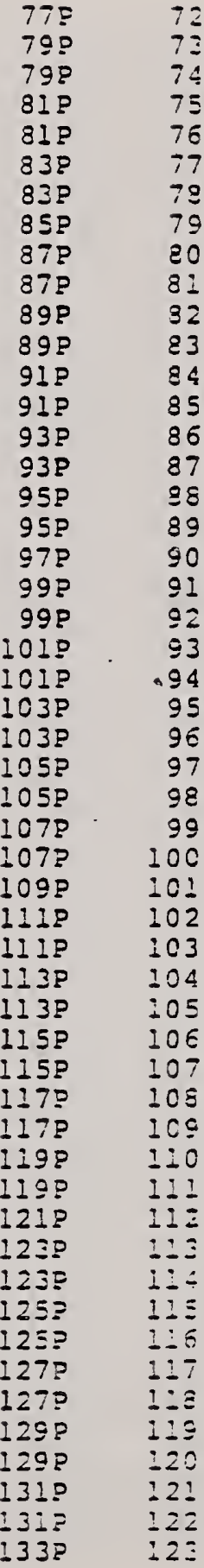


$212,1,6, .3349500, .0609000,1,1.5707963,0.0,0,0,7.0992059$, $5.4429245,0.0,6 \% 85.180,0,0$;

$214,1, .1500000, .0500000,0.0,7.5983694,5.6951459,7.5983694$, $5.6951460,0,0$;

$214,1, .1500000, .0500000,0.0,7.5555836,6.7116229,7.5555836$, $6.7115228,0,0$;

$106,1,3,0.0,7.3559408,5.8998650,7.3559408,5.8998650,7.6938728$, $5.6144979,0,0$;

$106,1,3,0.0,7.5426034,6.6986427,7.5426034,6.6986427,7.6439719$, $6.8000113,0,0$;

$202,135,141,143,7.0240726,6.1801114, .7516703,137,139,0,0$;

$212,1,7, .3897600, .0609000,1,1.5707963,0.0,0,0,1.3302597$,

$3.9236999,0.0,7 \pm 85.1792,0,0$;

$214,1, .1500000, .0500000,0.0,1.4018294,3.3481638,1.6171509$,

$3.8627899,0,0$;

$214,1, . .1500000, .0500000,0.0,1.3489565,4.6042754,1.6158312$,

$4.0454843,0,0$;

$106,1,3,0.0,1.0240077,3.6672140,1.0240077,3.6672140,1.4973330$, $3.2675161,0,0$;

$106,1,3,0.0,1.2106713,4.4659909,1.2106713,4.4659909,1.4373450$, $4.6926637,0,0$;

$202,147,153,155, .6921390,3.9474597, .9288789,149,151,0,0$;

$212,1,7, .3958500, .0609000,1,1.5707963,0.0,0,0,3.8625412$,

$4.0193701,0.0,7+185.1793,0,0 ;$

$214,1, .1500000, .0500000,0.0,3.8984568,3.2883423,3.7691025$,

$3.1593199,0,0$;

$214,1, .1500000, .0500000,0.0,3.8375103,4.7362208,3.6977707$,

$4.8539159,0,0$;

$106,1,3,0.0,3.4122883,3.6988844,3.4122883,3.6988844,3.9939605$, $3.2076946,0,0$;

$106 ; 1,3,0.0,3.5989513,4.4976620,3.5989513,4.4976620,3.9258987$, $4.8246092,0,0$;

$202,159,165,167,3.0804195,3.9791300,1.0706880,161,163,0,0$;

$212,1,7, .4019400, .0609000,1,1.5707963,0.0,0,0,5.0241055$,

$3.3770704,0.0,7485.1794,0,0$;

$214,2, .1500000, .0500000,0.0,5.9888410,3.5679451,5.7952855$,

$3.4075204,5.6452855,3.4075204,0,0$;

$214,1, .1 \equiv 00000, .0500000,0.0,5.9483098,4.5308470,5.8036153$,

$4.6423942,0,0$;

$106,1,3,0.0,5.7766815,3.7471037,5.7766815,3.7471037,6.0843445$, $3.4872973,0,0$;

$106,1,3,0.0,5.9533435,4.5458807,5.9633435,4.5458807,6.0366981$, $4.6192353,0,0$;

$202,171,177,170,5.4448123,4.0273495, .7120531,173,175,0,0$;

$212,1,6, .3288600, .0609000,1,1.5707963,0.0,0,0,6.8264465$,

$3.4030139,0.0,6 \mathrm{H} 85.179,0,0$;

$214,1, .1500000, .0500000,0.0,7.4360137,3.6497102,7.4360137$,

$3.6497103,0,0$ :

$214,1, .1500000, .0500000,0.0,7.4026967,4.4412337,7.4026967$,

$4.4412337,0,0$;

$106,1,3,0.0,7.3206811,3.7471029,7.3206811,3.7471029,7.5315171$, $3.5590623,0,0$; 
$106,1,3,0.0,7.5073437,4.5458807,7.5073437,4.5458807,7.4910850$, $4.5296221,0,0$;

$202,183,189,191,6.9888124,4.0273495, .5853207,185,187,0,0$;

$212,1,7, .3958500, .0609000,1,1.5707963,0.0,0,0,1.1058791$, $1.8199995,0.0,7 \pm 85.1793,0,0$;

$214,1, .1500000, .0500000,0.0,1.1659350,1.2216534,1.3919861$, $1.7590941,0,0$;

$214,1, .2500000, .0500000,0.0,1.1103433,2.5423430,1.3922339$, ..9: 17878,$0 ; 0$

$106,1,3,0.0, .7516278,1.5715137, .7516278,1.5715137,1.2614386$,

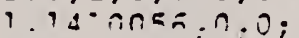

$106,1,3,0.0, .9382913,2.3702906, .9382913,2.3702906,1.1987316$, $2.5307313,0,0$;

$202,195,201,203, .4197590,1.8517594, .9766332,197,199,0,0$;

$212,1,7, .4019400, .0609000,1,1.5707963,0.0,0,0,3.4781609$,

$1.8836700,0.0,7485.1794,0,0$;

$2 i 4, i, .1500000, .0500000,0.0,3.5390949,1.2560912,3.4084319$,

$1.1383944,0,0$;

$214: 1, .1500000, .0500000,0.0,3.4846301,2.5600204,3.3436981$,

$2.6752850,0,0$;

$106,1,3,0.0,3.1399088,1.6031842,3.1399088,1.6031842,3.6345984$, $1.1854433,0,0$;

$106, i, 3,0.0,3.3265714,2.4019615,3.3265714,2.4019615,3.5730184$,

$2.6484088,0,0$;

$202,207,213,215,2.8080406,1.8834301, .9568427,209,211,0,0$;

$212,1,7, .3897600, .0609000,1,1.5707963,0.0,0,0,6.3890426$, 1. $4712123,0.0,7 \mathrm{~B} 35.1792,0,0$;

$214,1, .1500000, .0500000,0.0,5.1029579,2.3936551,5.2380745$,

$2.5165299,0,0$;

$214,2, .1500000, .0500000,0.0,5.1427211,1.4490310,6.1781426$,

$1.5015623,6.3281426,1.5016623,0,0$;

$106,1,3,0.0,5.5650342,2.0034576,5.5650342,2.0034576,5.0074541$,

$2.4743026,0,0$;

$106,1,3,0.0,5.5703717,1.8766816,5.5703717,1.8766816,5.0543327$,

$1.3606427,0,0$ :

$202,219,225,227,5.6366620,1.9429719, .6985381,221,223,0,0$;

$212,1,5, .328 \varepsilon 600, .0609000,1,1.5707963,0.0,0,0,7.3094230$,

$2.7152557,0.0,6485.179,0,0$;

$214,1, .1500000, .0500000,0.0,7.8516844,1.3763279,7.85 I 6846$,

$1.3753281,0,0$;

$214,1, .1500000, .0500000,0.0,7.8016917,2.5640013,7.8016917$,

2. $5040013,0,0$;

$106,1,3,0.0,7.5125309,1.6627255,7.5125309,1.6527255,7.9471879$,

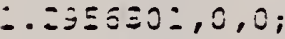

$106,1,3,0.0,7.6991934,2.4615030,7.6991934,2.4615030,7.8900300$, $2.652 \vdots 897,0,0$;

$202,231,237,239,7.1806622,1.9429715, .8782536,233,235,0,0$;

$212,1,7, .3952500, .0609000,1,1.5707963,0.0,0,0, .9173290, .0833981$, c. $1985 . \therefore 903,0,0$;

$21 \leq, 1, .1500000, .0500000,0.0,1.0071373,-.4867494,1.2085609$,

. $ن \angle 2 \Sigma \cup y \xi, 0,0$;

$2-\therefore, \ldots, 1500000, .0500000,0.0, .9582702, .6742085,1.1985497$,

1918

1918

$193 P$

$195 \mathrm{P}$

$195 \mathrm{P}$

1973

$197 P$

$199 \mathrm{P}$

$199 \mathrm{P}$

201P

$201 P$

203 ?

$203 P$

$205 P$

207?

207 ?

209P

$209 P$

211P

$211 P$

$213 P$

$213 \mathrm{P}$

$215 P$

$215 P$

2179

$219 \mathrm{P}$

$219 \mathrm{P}$

221 P

$221 \mathrm{P}$

223 P

223P

$225 P$

$225 P$

227?

227 P

$229 \mathrm{P}$

231?

231?

$233 \mathrm{P}$

$233 P$

$235 \mathrm{P}$

$235 P$

237 P

$237 ?$

2398

$239 \mathrm{~F}$

24I?

$243 P$

243 ?

245 ?

245?

2473
176

177

178

179

$18 \mathrm{C}$

181

182

183

184

185

186

187

188

189

190

191

192

193

194

195

196

197

iะ 8

199

200

201

202

203

204

205

206

207

208

209

210

211

212

213

214

215

216

21.

$21 \Xi$

215

220

221

222

225

226

225

22

2ะ? 
$.2052020,0,0$;

$106,1,3,0.0, .6830777,-.2130974, .6830777,-.2130974,1.1025408$,

$-.5673972,0,0$;

$106,1,3,0.0, .8697411, .5856799, .8697411, .5856799,1.0466586$,

$.7625968,0,0$;

$202,243,249,251, .3512089, .0671486, .8585134,245,247,0,0$;

$212,1,7, .4019400, .0609000,1,1.5707963,0.0,0,0,3.1773295$, $.1458504,0.0,7885.1794,0,0$;

$214,1, .1500000, .0500000,0.0,3.2839415,-.3561670,3.1502479$,

$-.4806873,0,0$;

$214,1, .1500000, .0500000,0.0,3.2403780, .6787810,3.0966931$,

$.7916258,0,0$;

$106,1,3,0.0,3.0310769,-.1426350,3.0310769,-.1426350,3.3794449$,

$-.4368140,0,0$;

$106,1,3,0.0,3.2177395, .6561427,3.2177395, .6561427,3.3297663$,

$.7671694,0,0$;

$202,255,261 ; 263,2.6992087, .1376110, .7653295,257,259,0,0$;

$212,1,7, .3958500, .0609000,1,1.5707963,0.0,0,0,4.7031802$,

$-.5973330,0.0,7485.1795,0,0$;

$214,2, .1500000, .0500000,0.0,5.6679149,-.3997418,5.4743603$,

$-.5668830,5.3243603,-.5668830,0,0$;

$214,1, .1500000, .0500000,0.0,5.6214326, .7045106,5.4786027$, $.8184356,0,0$;

$106,1,3,0.0,5.3758932,-.1531452,5.3758932,-.1531452,5.7634184$,

$-4803896,0,0$;

$106,1,3,0.0,5.5625551, .6456324,5.5625551, .6456324,5.7098208$,

$.7928991,0,0$;

$202,267,273,275,5.0440249, .1271007, .8165793,259,271,0,0$;

$212,1,6, .3288600, .0609000,1,1.5707963,0.0,0,0,6.8689485$,

$-.5844389,0.0,6385.179,0,0$;

$214,1, .1500000, .0500000,0.0,7.2677093,-.3763738,7.2677093$,

$-.3763738,0,0$;

$214,1, .1500000, .0500000,0.0,7.2215309, .7206866,7.2215309$,

$.7206866,0,0$;

$106,1,3,0.0,0.9797512,-.1332080,0.9797512,-.1332080,7.3632129$,

$-.4570216,0,0$;

$106,1,3,0.0,7.1664137, .6655694,7.1664137, .6655694,7.3099192$,

$.8090750,0,0$;

$202,279,285,287,6.6478825, .1470380, .8112615,281,283,0,0$;

$S \quad 1 G \quad 20 \quad 290 \mathrm{P} 266$ 


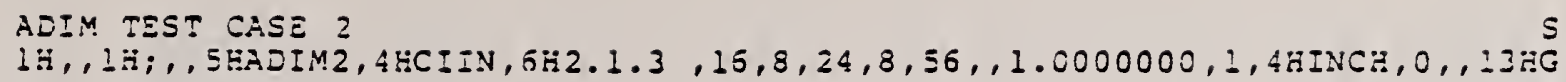
$810827.122507 \ldots$. . ;

$\begin{array}{llll}124 & 1 & 1 & 1 \\ 124 & 0 & 0 & 2 \\ 212 & 3 & 1 & 1 \\ 212 & 0 & 0 & \cdot \\ 214 & 5 & 1 & 1 \\ 214 & 0 & 0 & 2 \\ 214 & 7 & 1 & 1 \\ 214 & 0 & 0 & 2 \\ 106 & 9 & 1 & 1 \\ 106 & 0 & 0 & 2 \\ 106 & 11 & 1 & 1 \\ 106 & 0 & 0 & 2 \\ 202 & 13 & 1 & 1 \\ 202 & 0 & 0 & 1 \\ 212 & 14 & 1 & 1 \\ 212 & 0 & 0 & 2 \\ 214 & 16 & 1 & 1 \\ 214 & 0 & 0 & 2 \\ 214 & 18 & 1 & 1 \\ 214 & 0 & 0 & 2 \\ 106 & 20 & 1 & 1 \\ 106 & 0 & 0 & 2 \\ 106 & 22 & 1 & 1 \\ 106 & 0 & 0 & 2 \\ 202 & 24 & 1 & 1 \\ 202 & 0 & 0 & 1 \\ 212 & 25 & 1 & 1 \\ 212 & 0 & 0 & 2 \\ 214 & 27 & 1 & 1 \\ 214 & 0 & 0 & 2 \\ 214 & 29 & 1 & 1 \\ 214 & 0 & 0 & 2 \\ 106 & 31 & 1 & 1 \\ 105 & 0 & 0 & 2 \\ 106 & 33 & 1 & 1 \\ 106 & 0 & 0 & 2 \\ 202 & 35 & 1 & 1 \\ 202 & 0 & 0 & 1 \\ 212 & 36 & 1 & 1 \\ 212 & 0 & 0 & 2 \\ 214 & 38 & 1 & 1 \\ 214 & 0 & 0 & 2 \\ 214 & 40 & 1 & 1 \\ 214 & 0 & 0 & 2 \\ 106 & 42 & 1 & 1 \\ 106 & 0 & 0 & 2 \\ 106 & 44 & 1 & 1 \\ 106 & 0 & 0 & 2 \\ 202 & 46 & 1 & 1 \\ & & & \end{array}$
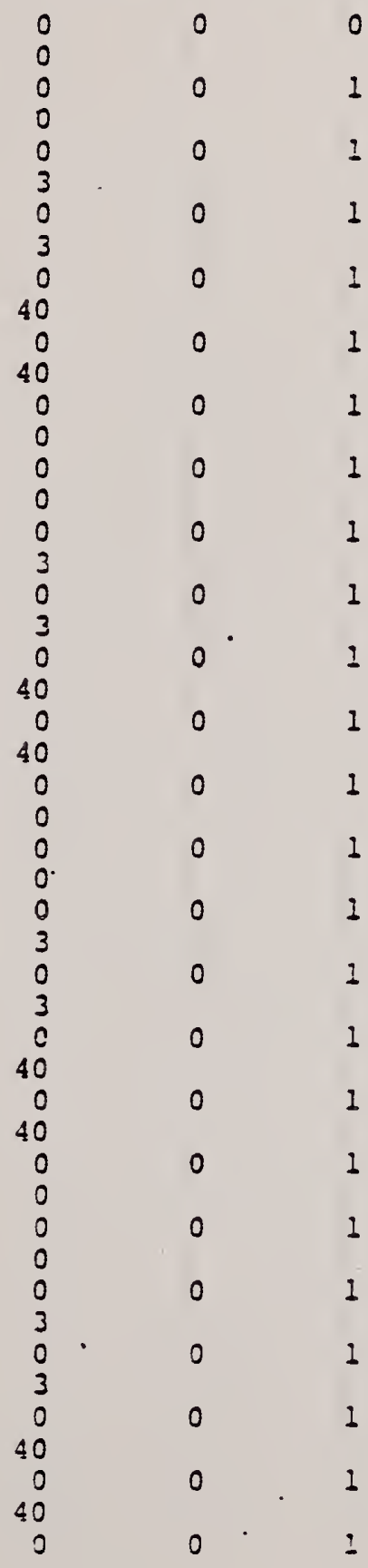

$\begin{array}{lllll}0 & 0 & 0 & 0 & D\end{array}$

$\begin{array}{lllllll}0 & 0 & 1 & 1 & 0 & 0 \\ 0\end{array}$

$\begin{array}{llllll}0 & 0 & 1 & 1 & 0 & D\end{array}$

$\begin{array}{llllll}0 & 0 & 1 & 1 & 0 & 0\end{array}$

$\begin{array}{lllll}0 & 1 & 1 & 1 & D \\ 0 & & & 0 & 0 \\ 0 & 1 & 1 & 1 & D\end{array}$

$\begin{array}{llllllllll}0 & 1 & 1 & 1 & D\end{array}$

$\begin{array}{llllllll}0 & 0 & 0 & 1 & D\end{array}$

$\begin{array}{llllll}0 & 0 & 1 & 1 & 0 & 0\end{array}$

$\begin{array}{lllll}0 & 0 & 1 & 1 & 0 D\end{array}$

OD

$\begin{array}{llllll}0 & 0 & 1 & 1 & D & 0\end{array}$

106

106

202

202

214

214

214

106

106

212

212

214

214

106

106

202

46

1

0

$\begin{array}{lllll}0 & 1 & 1 & 1 & D\end{array}$

OD 22

101110023

$1 \quad 0001025$

$1 \quad 00110 D \quad 27$

100110029

1001100

1001100

$1,0 D \quad 34$

$\begin{array}{llll}10 & 0 & 0 & 36\end{array}$

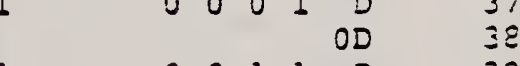

$\begin{array}{llllll}0 & 0 & 1 & 1 & 0 & \\ 0 & 0 & 1 & 0 & 0\end{array}$

001,0042

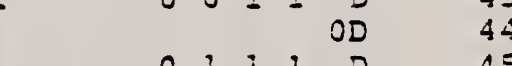

O.1- OD $4 E$

$\begin{array}{llll}1 & 011100 & 48 \\ 1 & 000 \div 0 & 45\end{array}$ 


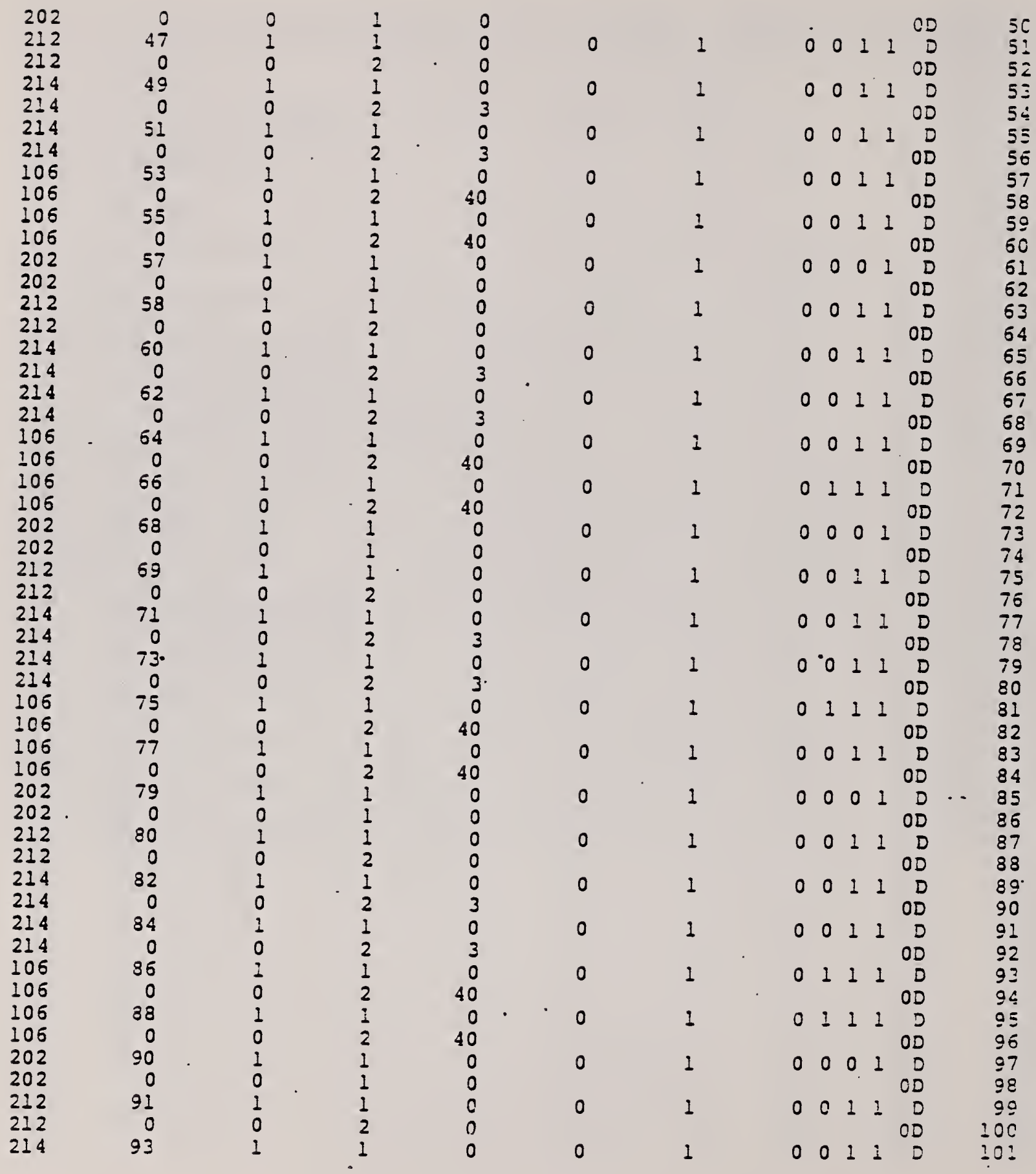




\begin{tabular}{|c|c|c|c|c|c|c|c|c|c|c|c|}
\hline $\begin{array}{l}214 \\
214\end{array}$ & 95 & $\begin{array}{l}0 \\
1\end{array}$ & $\begin{array}{l}2 \\
1\end{array}$ & $\begin{array}{l}3 \\
0\end{array}$ & 0 & 1 & 0 & 0 & 1 & & $\begin{array}{l}D \\
D\end{array}$ \\
\hline $21 \frac{1}{2}$ & 0 & 0 & 2 & 3 & & & & & & & $D$ \\
\hline$: 06$ & 97 & 1 & 1 & 0 & 0 & 1 & 0 & 0 & I & 1 & $D$ \\
\hline 106 & 0 & 0 & 2 & 40 & & & & & & & $D$ \\
\hline 106 & 99 & 1 & 1 & 0 & 0 & 1 & 0 & 0 & I & 1 & $D$ \\
\hline$\therefore 06$ & 0 & 0 & 2 & 40 & & & & & & & $D$ \\
\hline 202 & 101 & 1 & 1 & 0 & 0 & 1 & 0 & 0 & 0 & 1 & $D$ \\
\hline 202 & & 0 & 1 & 0 & & & & & & & D \\
\hline $2 ! 2$ & $\begin{array}{r}102 \\
0\end{array}$ & 1 & $\frac{1}{2}$ & 0 & 0 & 1 & 0 & 0 & 1 & 1 & D \\
\hline $\begin{array}{r}212 \\
214\end{array}$ & 104 & 1 & 1 & $\begin{array}{l}0 \\
0\end{array}$ & 0 & 1 & 0 & 0 & 1 & 1 & D \\
\hline 214 & 0 & 0 & 2 & 3 & & & & & & & \\
\hline $2: 4$ & 106 & 1 & 1 & 0 & 0 & 1 & 0 & 0 & 1 & 1 & D \\
\hline 214 & 0 & 0 & 2 & 3 & & & & & & & \\
\hline 106 & 108 & 1 & 1 & 0 & 0 & 1 & 0 & 0 & 1 & 1 & D \\
\hline 106 & 0 & 0 & 2 & 40 & & & & & & & \\
\hline 106 & 110 & 1 & 1 & 0 & 0 & 1 & 0 & 0 & 1 & 1 & $D$ \\
\hline 106 & 0 & 0 & 2 & 40 & & & & & & & \\
\hline 202 & 112 & 1 & 1 & 0 & 0 & 1 & 0 & 0 & 0 & & ? \\
\hline 202 & 0 & 0 & 1 & 0 & & & & & & & \\
\hline 212 & 113 & 1 & 1 & 0 & 0 & 1 & 0 & 0 & & & D \\
\hline 212 & 0 & 0 & 2 & 0 & & & & & & & \\
\hline 214 & 115 & 1 & 1 & 0 & 0 & 1 & 0 & 0 & & $b$ & D \\
\hline 214 & 0 & 0 & 2 & 3 & & & & & & & \\
\hline 214 & $1: 7$ & 1 & 1 & 0 & 0 & 1 & 0 & 0 & 1 & $b$ & D \\
\hline 214 & 0 & 0 & 2 & 3 & & & & & & & \\
\hline 106 & 119 & 1 & 1 & 0 & 0 & 1 & 0 & 0 & & & D \\
\hline 106 & 0 & 0 & 2 & 40 & & & & & & & \\
\hline 106 & 221 & 1 & 1 & 0 & 0 & 1 & 0 & 0 & 1 & 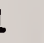 & D \\
\hline ¿úó & $\hat{u}$ & 0 & 2 & 40 & & & & & & & \\
\hline 202 & 123 & 1 & 1 & 0 & 0 & 1 & 0 & 0 & 0 & 1 & E \\
\hline 202 & 0 & 0 & 1 & 0 & & & & & & & \\
\hline $\begin{array}{l}212 \\
212\end{array}$ & $\begin{array}{r}124 \\
0\end{array}$ & $\begin{array}{l}1 \\
0\end{array}$ & $\frac{1}{2}$ & $\begin{array}{l}0 \\
0\end{array}$ & 0 & 1 & 0 & 0 & 1 & & \\
\hline 214 & 126 & 1 & 1 & 0 & 0 & 1 & 0 & 0 & ? & & b \\
\hline $2 \geq 4$ & 0 & 0 & 2 & 3 & & & & & & & \\
\hline 214 & 128 & 1 & $\frac{1}{0}$ & 0 & 0 & 1 & 0 & 0 & 1 & & $D$ \\
\hline 214 & 0 & 0 & 2 & 3 & & & & & & 0 & \\
\hline & $\begin{array}{r}1 \geq 0 \\
0\end{array}$ & $\begin{array}{l}1 \\
0\end{array}$ & $\frac{1}{2}$ & 0 & 0 & 1 & 0 & 0 & 1 & & 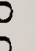 \\
\hline 206 & $\begin{array}{r}0 \\
1=2\end{array}$ & 1 & 1 & 40 & 0 & 1 & & & & & \\
\hline $\begin{array}{l}106 \\
100\end{array}$ & $\begin{array}{r}132 \\
0\end{array}$ & 0 & 2 & 0 & 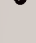 & 1 & 0 & 0 & 1 & & 0 \\
\hline 100 & $\begin{array}{r}0 \\
=4\end{array}$ & & & 40 & 0 & & & & & & \\
\hline 202 & 134 & 1 & 1 & 0 & v & 1 & 0 & 0 & 0 & & D \\
\hline 202 & 0 & 0 & 1 & 0 & & & & & & 0 & \\
\hline $2 \div 2$ & 235 & 1 & 1 & 0 & 0 & 1 & 0 & 0 & 1 & & D \\
\hline 212 & 0 & 0 & 2 & 0 & & & & & & 0 & \\
\hline $22 \frac{1}{2}$ & 137 & 1 & 1 & 0 & 0 & 1 & 0 & 0 & 1 & & D \\
\hline $2 \geq 4$ & 0 & 0 & 2 & 3 & & & & & & 0 & \\
\hline $2+4$ & 139 & 1 & 1 & 0 & 0 & 1 & 0 & 0 & 1 & & D \\
\hline 224 & 0 & 0 & 2 & 3 & & & & & & & D \\
\hline$: 06$ & 149 & 1 & 1 & 0 & 0 & $i$ & 0 & 1 & 1 & & b \\
\hline
\end{tabular}




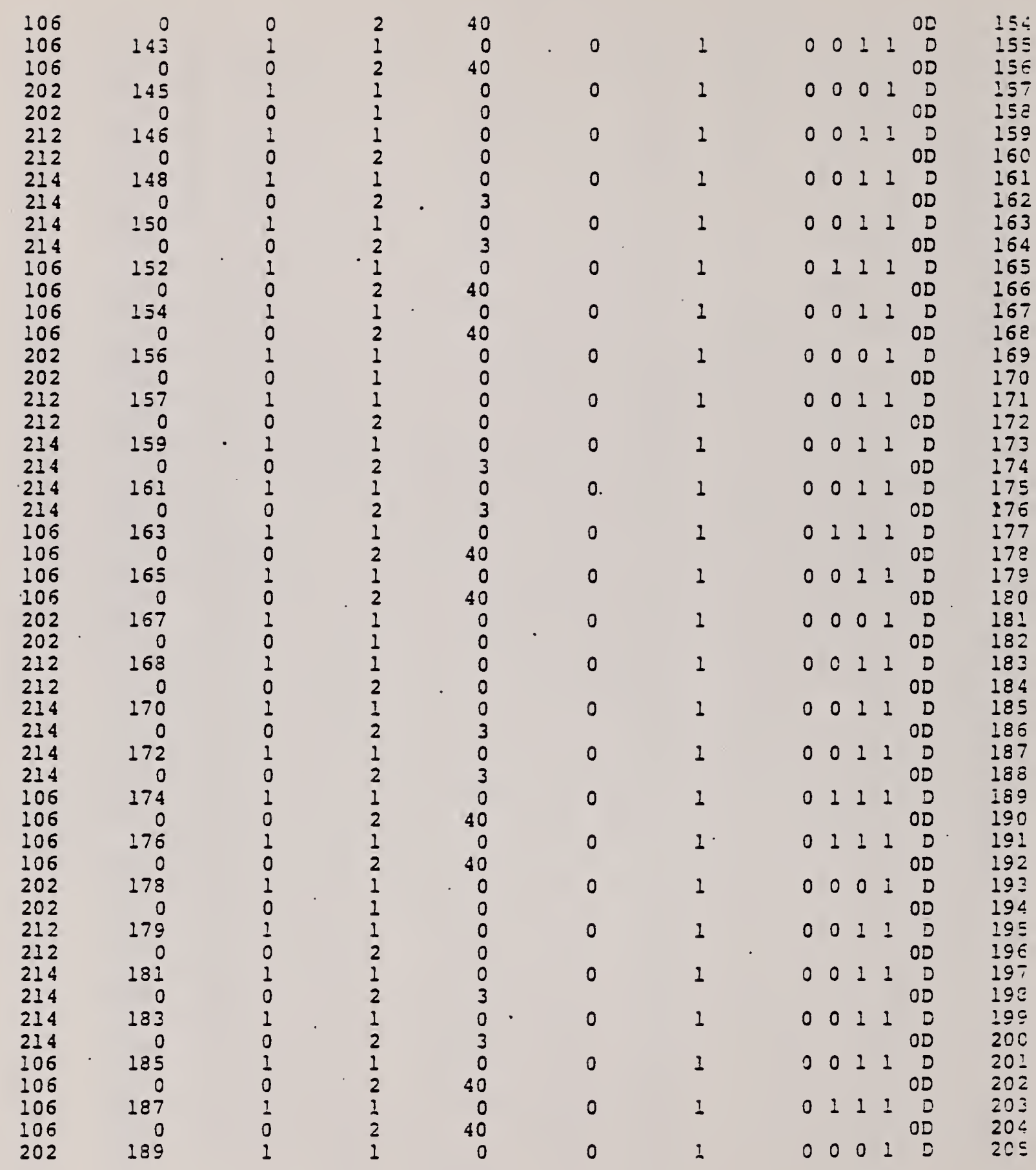




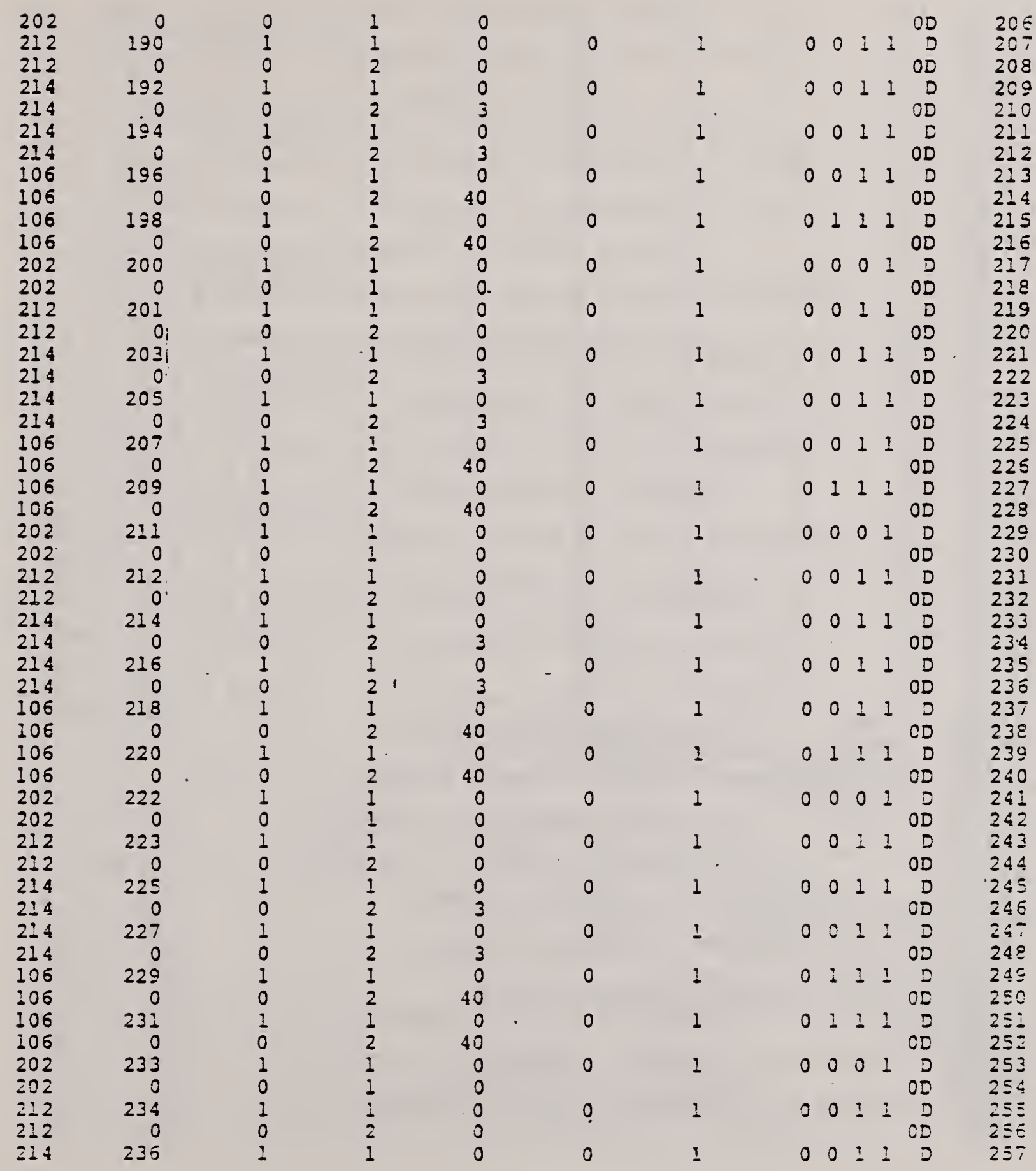




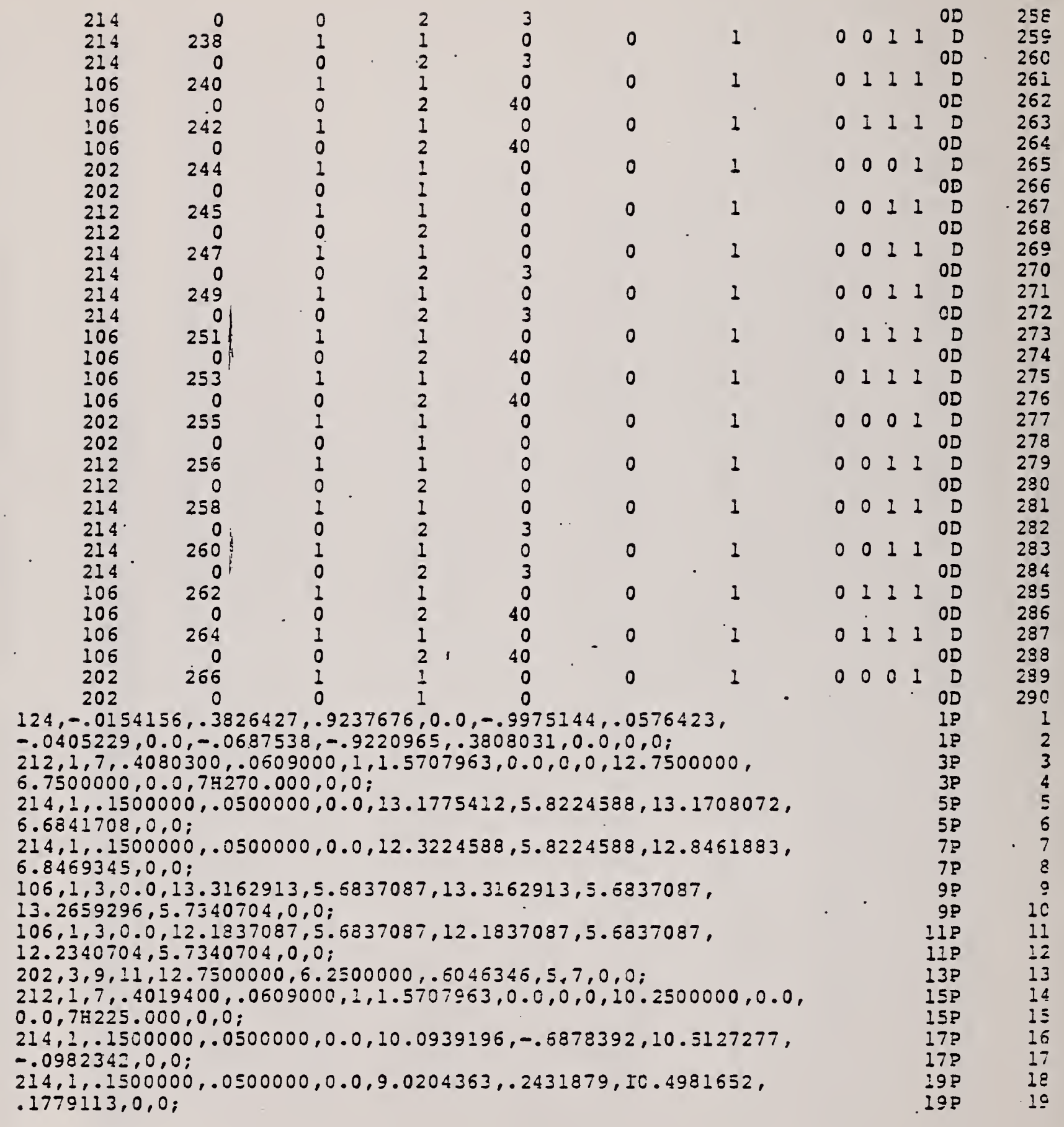


$106,1,3,0.0,10.0419263,-.5838525,10.0419263,-.5838525$, $10.1498213,-.7996426,0,0$

$106,1,3,0.0,8.9110009, .2796464,8.9110609, .2795464,8.9018509$, $.2827164,0,0$;

$202,15,21,23,9.7500000,0.0, .7690277,17,19,0,0$;

$212,1,7, .4019400, .0609000,1,1.5707963,0.0,0,0,10.5000000$,

$2.0000000,0.0,7 \mathrm{E} 225.000,0,0$;

$214,1, .1500000, .0500000,0.0,10.2056059,1.0887882,10.7639457$, $1.9010157,0,0$;

$214,1, .1500000, .0500000,0.0,8.7835139,2.3221620,10.7528776$,

$2.1792215,0,0 ;$

$106,1,3,0.0,10.0419263,1.4161475,10.0419263,1.4161475$,

$10.2515076, .9769848,0,0$;

$106,1,3,0.0,8.9110609,2.2796464,8.9110609,2.2796464,8.6649285$, $2.3616905,0,0$;

$202,27,33,35,9.7500000,2.0000000,1.0187658,29,31,0,0$;

$212,1,7, .4019400, .0609000,1,1.5707963,0.0,0,0,10.2500000$,

$4.0000000,0.0,7$ H $225.000,0,0$;

$214,1, .1500000, .0500000,0.0,10.0939196,3.3121608,10.5127277$,

$3.9017660,0,0$;

$214,1, .1500000, .0500000,0.0,9.0204363,4.2431879,10.4981651$,

$4.1779126,0,0$;

$106,1,3,0.0,10.0419263,3.4161475,10.0419253,3.4161475$,

$10.1498213,3.2003574,0,0$;

$106,1,3,0.0,3.9110609,4.2796464,8.9110509,4.2796464,8.9018509$, $4.2827164,0,0$;

$202,39,45,47,9.7500000,4.0000000, .7690277,41,43,0,0$;

$212,1,7, .4019400, .0609000,1,1.5707963,0.0,0,0,10.2500000$,

$6.5000000,0.0,78225.000,0,0$;

$214,1, .1500000, .0500000,0.0,10.1171928,5.5156143,10.5564698$,

$6.4041407,0,0$;

$214,1, .1500000, .0500000,0.0,3.9710654,6.5096446,10.4565812$,

$6.5682056,0,0$ :

$106,1,3,0.0,10.0419263,5.6661475,10.0419263,5.6661475$,

$10.1730945,5.4038109,0,0$;

$106,1,3,0.0,8.9110509,0.5296464,8.9110609,6.5296464,8.8524810$,

$6.5491730,0,0$;

$202,51,5 ?, 59,9.7500000,6.2500000, .8210682,53,55,0,0$;

$212,1,7, .4080300, .0609000,1,1.5707963,0.0,0,0,12.7500000$,

$2.7500000,0.0,7 \mathrm{H} 270.000,0,0$;

$214,1, .1500000, .0500000,0.0,13.3409027,1.4090973,13.2171124$,

$2.6929199,0,0$;

$214,1, .1500000, .0500000,0.0,12.1590973,1.4090973,12.8062794$,

$2.8337653,0,0$;

$106,1,3,0.0,13.3162913,1.4337087,13.3162913,1.4337087$,

$13.4292910,1.2207090,0,0$;

$106,1,3,0.0,12.1837087,1.4337087,12.1837087,1.4337087$,

$12.0707090,1.3207090,0,0$;

$202,63,69,71,12.7500000,2.0000000, .8356626,65,67,0,0$;

$212,1,7, .4080300, .0609000,1,1.5707963,0.0,0,0,12.7500000$,

$4.7500000,0.0,792=0.000,0,0$;

$214,1, .1500000, .0500000,0.0,13.3409028,3.4090972,13.2171053$,

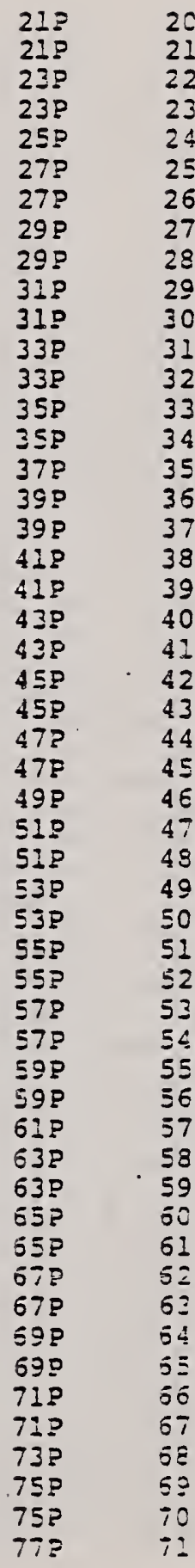


$4.6929249,0,0$;

$214,1, .1500000, .0500000,0.0,12.1590972,3.4090972,12.8062877$, $4.8337649,0,0$;

$105,1,3,0.0,13.3162913,3.4337087,13.3162913,3.4337087$,

$13.4292912,3.3207088,0,0$;

$106,1,3,0.0,12.1837087,3.4337087,12.1837087,3.4337087$,

$12.0707088,3.3207088,0,0$;

$202,75,81,83,12.7500000,4.0000000, .8356628,77,79,0,0$;

$212,1,7, .4080300, .0609000,1,1.5707963,0.0,0,0,12.7500000$,

$.7500000,0.0,74270.000,0,0 ;$.

$214,1, .1500000, .0500000,0.0,13.3409027,-.5909027,13.2171124$,

$.6929199,0,0$;

$214,1, .1500000, .0500000,0.0,12.1590973,-.5909027,12.8062794$,

$.8337653,0,0$;

$106,1,3,0.0,13.3162913,-.5662913,13.3162913,-.5662913$,

$13.4292910,-.6792910,0,0$;

$106,1,3,0.0,12.1837087,-.5662913,12.1837087,-.5662913$,

$12.0707090,-.6792910,0,0$;

$202,87,93,95,12.7500000,0.0, .8356626,89,91,0,0$;

$212,1,7, .4019400,00609000,1,1.5707963,0.0,0,0,1.3335195$, $6.0924616,0.0,7385.1794,0,0$;

$214,1, .1500000,00500000,0.0,1.4129652,5.5212946,1.6220727$,

$6.0315438,0,0$;

$214,1, .1500000, .0500000,0.0,1.3618900,6.7347125,1.6174298$,

$6.2142524,0,0$;

$106,1,3,0.0,1.0592672,5.8199757,1.0592672,5.8199757,1.5084687$, $5.4406467,0,0$;

$106,1,3,0.0,1.2459306,6.6187529,1.2459306,6.6187529,1.4502783$, $6.8231009,0,0$;

$202,99,105,107, .7273991,6.1002216, .8973057,101,103,0,0 ;$

$212,1,7, .3958500,00609000,1,1.5707963,0.0,0,0,3.7853014$,

$6.1401320,0.0,7585.1795,0,0$

$214,1, .1500000, .0500000,0.0,3.8469553,5.5143649,3.7162957$,

$5.3866646,0,0$;

$214,1, .1500000, .0500000,0.0,3.7924764,6.8086880,3.6515481$, $6.9249570,0,0$;

$106,1,3,0.0,3.4475477,5.8516462,3.4475477,5.3516462,3.9424585$, $5.4337167,0,0$;

$106,1,3,0.0,3.6342111,6.6504234,3.6342111,6.6504234,3.8808649$, $6.8970763,0,0$ :

$202,111,117,119,3.1156802,6.1318932, .9571335,113,115,0,0$;

$212,1,7, .3958500, .0609000,1,1.5707963,0.0,0,0,5.4941936$,

$5.5083507,0.0,7585.1797,0,0$;

$214,1, .1500000, .0500000,0.0,4.9675483,6.6129165,5.1033343$,

$6.7351517,0,0$

$214,2, .1500000, .0500000,0.0,5.0057302,5.7057691,5.2832936$,

$5.5388007,5.4332936,5.5388007,0,0$ :

$106,1,3,0.0,5.4084452,6.2405977,5.4084452,6.2405977,4.8720451$, $6.6935649,0,0$;

$106,1,3,0.0,5.4137822,6.1138211,5.4137822,6.1138211,4.9173418$, $5.6173807,0,0$;

$202,123,129,131,5.4800725,6.1801114, .6708214,125,127,0,0$;

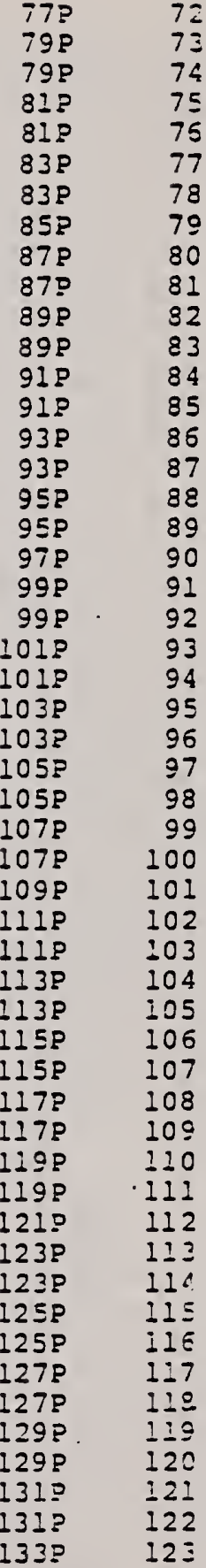


$212,1,6, .3349500, .0609000,1,1.5707963,0.0,0,0,7.0992069$, $\Xi .4429245,0.0,6785.180,0,0$;

$2 \pm, 1, .5000000, .0500000,0.0,7.5983694,5.6951459,7.5983694$,

$5.6951460,0,0$;

$214,1, .1500000,00500000,0.0,7.5555836,6.7116229,7.5555836$,

$6.7116228,0,0$;

$106,1,3,0.0,7.3559408,5.8998650,7.3559408,5.8998650,7.6938728$,

$5.6144979,0,0$;

$106,2,3,0.0,7.5426034,6.6986427,7.5426034,6.6986427,7.6439719$, $6.8000113,0,0$;

$202,135,141,143,7.0240726,5.1801114, .7516703,137,139,0,0$;

$212,1,7, .3897500, .0609000,1,1.5707963,0.0,0,0,1.3302597$,

$3.9235999,0.0,7485.1792,0,0$;

$=14,1, .5000000, .0500000,0.0,1.4018294,3.3481638,1.6171509$,

$3.8627899,0,0$;

$214,1, .1500000, .6500000,0.0,1.3489565,4.6042754,1.6158312$,

$4.0454843,0,0$;

$106,1,3,0.0,1.0240077,3.6572140,1.0240077,3.6672140,1.4973330$, $3.2675161,0,0$;

$106,1,3,0.0,1.2106713,4.4659909,1.2106713,4.4659909,1.4373450$, $4.6925637,0,0$;

$202,147,153,155, .6921390,3.9474597, .9288789,149,151,0,0$;

$212,1,7, .3 \equiv 58500, .0609000,1,1.5707963,0.0,0,0,3.8625412$,

$4.019370,1,0.0,7185.1793,0,0$

$214,1, .1500000, .0500000,0.0,3.8984568,3.2883423,3.7691025$,

$3.1593195,0,0$;

$214,1, .1500000, .0500000,0.0,3.8375103,4.7362208,3.6977707$,

$4.8539159,0,0$;

$106,1,3,0.0,3.4122883,3.6988844,3.4122883,3.6988844,3.9939605$, $3.2076946,0,0$;

+Uo, t, s,u.u, J.5989513,4.4976620,3'.5989513,4.4976520,3.9258987, $4.8246092,0,0$;

$202,159,: 55,167,3.0804195,3.9791300,1.0706880,161,163,0,0$;

$212,1,7, .4013400, .0609000,1,1.5707963,0.0,0,0,5.0241055$,

$3.3770704,0.0,7 \mathrm{H} 85.1794,0,0$;

$214,2, .1500000, .0500000,0.0,5.9888410,3.5579451,5.7952855$,

$3.4075204,5.6452855,3.4075204,0,0$;

$214,1, .1500000, .0500000,0.0,5.9483098,4.5308470,5.8036153$,

$4.6423942,0,0$;

$106,1,3,0.0,5.7766815,3.7471037,5.7765815,3.7471037,6.0843445$, $3.4872973,0,0$;

$105,1,3,0.0,5.9633435,4.5458807,5.9533435,4.5458807,6.0366981$, $4.5192353,0.0$;

$=02,7,1,77,179,5.4448123,4.0273495, .7120 \equiv 31,173,175,0,0$;

$212,1,6, .3288600, .0609000,1,1.5707963,0.0,0,0,6.8264465$,

¿. $\div 030 \div=0,0.0,6 \mathrm{H} 85.179,0,0$;

$214,1.1500000,00500000,0.0,7.4360137,3.6497102,7.4360137$,

$3.549 \div 203,0,0 ;$

$214.1, .1500000, .0500000,0.0,7.4026967,4.4412337,7.4026967$,

$4.4412327,0,0$;

$: 0 \sum, \therefore, 2,0.0,7.3206811,3.7471029,7.3206811,3.7471029,7.5315171$, $2.5590522,0,0$;

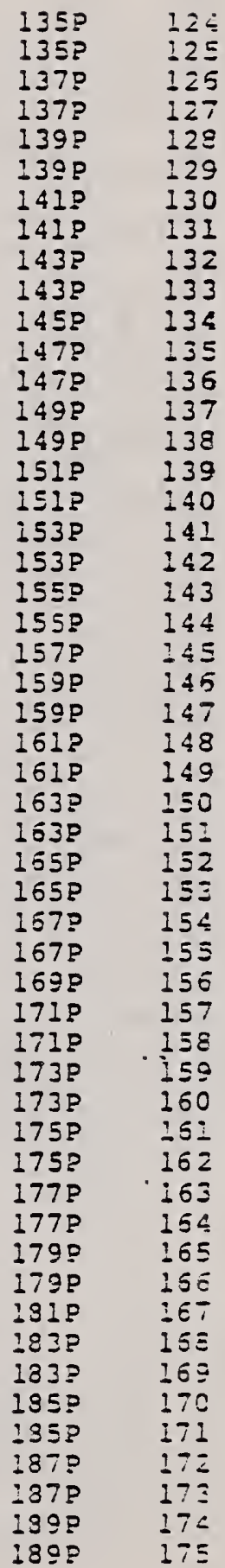


$106,1,3 ., 0.0,7.5073437,4.5458807,7.5073437,4.5458807,7.4910850$, $4.5296221,0,0$;

$202,183,189,191,6.9888124,4.0273495, .5853207,185,187,0,0$;

$212,1,7, .3958500, .0609000,1,1.5707963,0.0,0,0,1.1058791$,

$1.8199995,0.0,7 \mathrm{H85} .1793,0,0$;

$214,1, .1500000,00500000,0.0,1.1659350,1.2216534,1.3919861$, $1.7590941,0,0$;

$214,1, .1500000, .0500000,0.0,1.1103433,2.5423430,1.3922339$, $1.9417878,0,0$;

$106,1,3,0.0,07515278,1.5715137, .7516278,1.5715137,1.2614386$, $1.1410056,0,0$;

$106,1,3,0.0, .9382913,2.3702906, .9382913,2.3702906,1.1987316$, $2.6307313,0,0$;

$202,195,201,203, .4197590,1.8517594, .9766332,197,199,0,0 ;$

$212,1,7, .4019400 ; .0609000,1,1.5707963,0.0,0,0,3.4781609$, $1.8836700,0.0,7485.1794,0,0$;

$214,1, .1500000,00500000,0.0,3.5390949,1.2660912,3.4084319$, $1.1383944,0,0$;

$214,1, .1500000,00500000,0.0,3.4846301,2.5600204,3.3436981$, $2.6762850,0,0$;

$106,1,3,0.0,3.1399088,1.6031842,3.1399088,1.6031842,3.6345984$, $1.1854433,0,0$;

$106,1,3,0.0,3.3265714,2.401961 .5,3.3265714,2.4019615,3.5730184$, $2.548 \div 088,0,0$;

$202,207,213,215,2.3080406,1.8834301, .9568427,209,211,0,0$;

$212,1,7, .3897600,00609000,1,1.5707963,0.0,0,0,6.3890426$,

$1.4712123,0.0,71885.1792,0,0 ;$

$214,1, .1500000,00500000,0.0,5.1029579,2.3936551,5.2380745$,

$2.5166290,0,0$;

$214,2, .1500000,00500000,0.0,5.1427211,1.4490310,6.1781425$, $1.5016623,6.3281426,1.5016623,0,0 \%$

$106,1,3,0.0,5.5650342,2.0034576, \equiv .5650342,2.0034576,5.0074541$, $2.4743026,0,0$;

$106,1,3,0.0,5.5703717,1.8766816,5.5703717,1.8766816,5.0543327$, $1.3606427,0,0$;

$202,219,225,227,5.6366620,1.9429719, .6985381,221,223,0,0$;

$212,1,6, .3288600, .0609000,1,1.5707963,0.0,0,0,7.3094230$,

$2.7152557,0.0,6 \mathrm{~B} 85.179,0,0 ;$

$214,1, .1500000, .0500000,0.0,7.8515844,1.3763279,7.8516846$, $1.3763281,0,0$;

$214,1, .1500000,00500000,0.0,7.8016917,2.5640013,7.8015917$, $2.5640013,0,0$;

$106,1,3,0.0,7.5125309,1.6627255,7.5125309,1.6627255,7.9471879$, $1.2956801,0,0$;

$106,1,3,0.0,7.6991934,2.4615030,7.6991934,2.4615030,7.3900800$, $2.6523897,0,0$;

$202,231,237,239,7.18066 \cdot 22,1.9429715, .8782686,233,235,0,0$;

$212,1,7, .3958500, .0609000,1,1.5707963,0.0,0,0, .9173290,0.0833881$, $0.0,7 \xi 85.1793,0,0$;

$214,1, .1500000,00500000,0.0,1.0071373,-.4867494,1.2085609$, $.0225094,0,0$;

$214,1,1500000,00500000,0.0, .9582702, .6742086,1.1985497$,

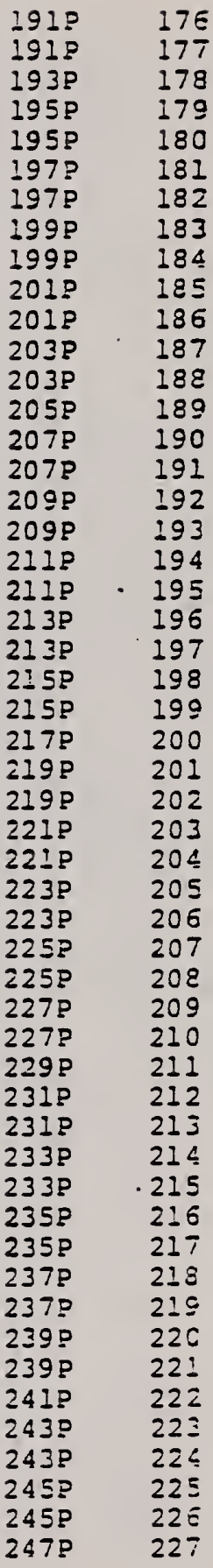


$.2052020,0,0$;

$105,1,3,0.0, .5830777,-.2130974, .6830777,-.2130974,1.1026408$,

$-.5673972,0,0$;

$249 ?$

$106,1,3,0.0, .8697411, .5856799, .8697411, .5856799,1.0466586$,

2518

$.7625958,0,0$;

$202,243,249,251, .3512089, .0671486, .8585134,245,247,0,0$;

$212,1,7, .4019400,00609000,1,1.5707963,0.0,0,0,3.1773295$,

$.1458504,0.0,7485.1794,0,0$;

$214,1, .1500000, .0500000,0.0,3.2839415,-.3561670,3.1502479$,

$-.4806873,0,0$;

$214,1, .1500000, .0500000,0.0,3.2403780, .5787810,3.0966931$,

$.7916258,0,0$;

$106,1,3,0.0,3.0310769,-.1426350,3.0310769,-.1426350,3.3794449$,

2519

2539

$255 P$

$255 P$

2579

$257 \mathrm{P}$

$259 P$

$259 P$

$-.4368149,0,0$;

$106,1,3,0.0,3.2177395, .6561427,3.2177395, .6561427,3.3287663$, $.7671694,0,0$;

$202,255,251,263,2.6992087, .1376110, .7653295,257,259,0,0$;

$212,1,7, .3958500, .0609000,1,1.5707963,0.0,0,0,4.7031802$,

$-.5973330,0.0,7385.1795,0,0 ;$

$214,2, .1500000, .0500000,0.0,5.6679149,-.3997418,5.4743603$,

$-.5668830,5.3243603,-.5668830,0,0$ i

$214,1, .1500000, .0500000,0.0,5.6214326, .7045106,5.4786027$,

$.8184356,0,0$;

$106,1,3,0.0,5.3758932,-.1531452,5.3758932,-.1531452,5.7634184$,

$-.4803896,0,0$;

$106,1,3,0.0,5.562555 i, .6456324,5.5625551, .6456324,5.7098208$,

$.7928991,0,0$

$202,267,273,275,5.0440249, .1271007, .8165793,259,271,0,0$;

$212,1,6, .3288600, .0609000,1,1.5707963,0.0,0,0,6.8689485$,

$-.5844389,0.0,6485.179,0,0$;

$214,1, .1500000, .0500000,0.0,7.2677093,-.3763738,7.2677093$,

$-.3763738,0,0$

$214,1, .1500000, .0500000,0.0,7.2215309, .7206866,7.2215309$,

$.7206866,0,0$;

$106,1,3,0.0,6.97975 i 2,-.1332080,6.9797512,-.1332080,7.3632129$,

$-.4570216,0,0$

$106,1,3,0.0,7.1664137, .6655694,7.1664137, .6655694,7.3099192$,

$.3090750,0,0$;

$202,279,285,287,6.6478825, .1470380, .8112615,231,283,0,0$;

$S \quad I G \quad 2 D \quad 290 P \quad 266$

261:

261P

263P

263 P

265P

267 P

267 P

2699

2695

2719

271 ?

273 P

273 P

275 P

275 P

2779

$279 P$

$279 \mathrm{P}$

$281 ?$

281 ?

$283 \mathrm{P}$

283 P

$285 P$

$285 \mathrm{P}$

$287 ?$

287 P

$289 \mathrm{P}$ 


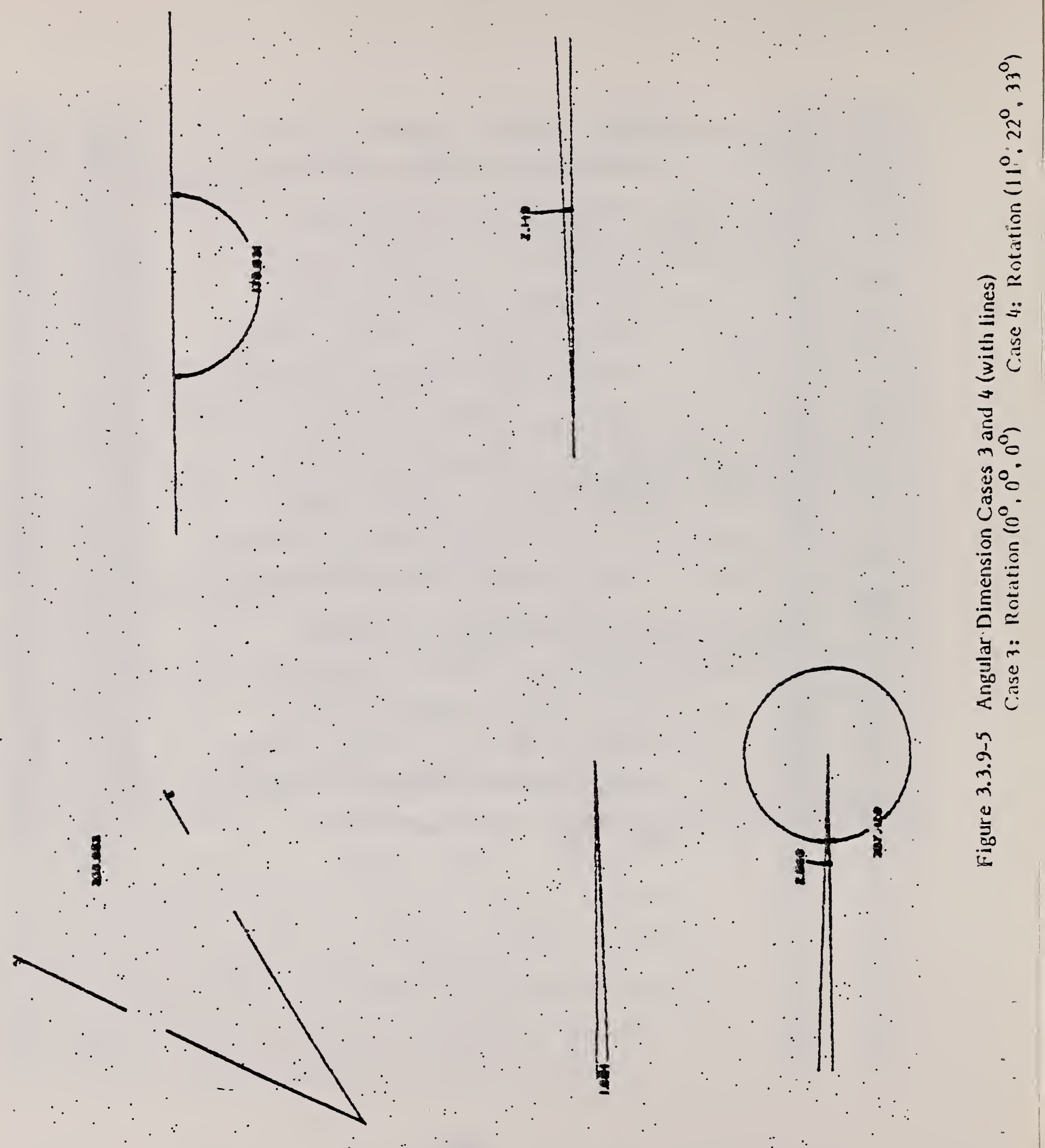




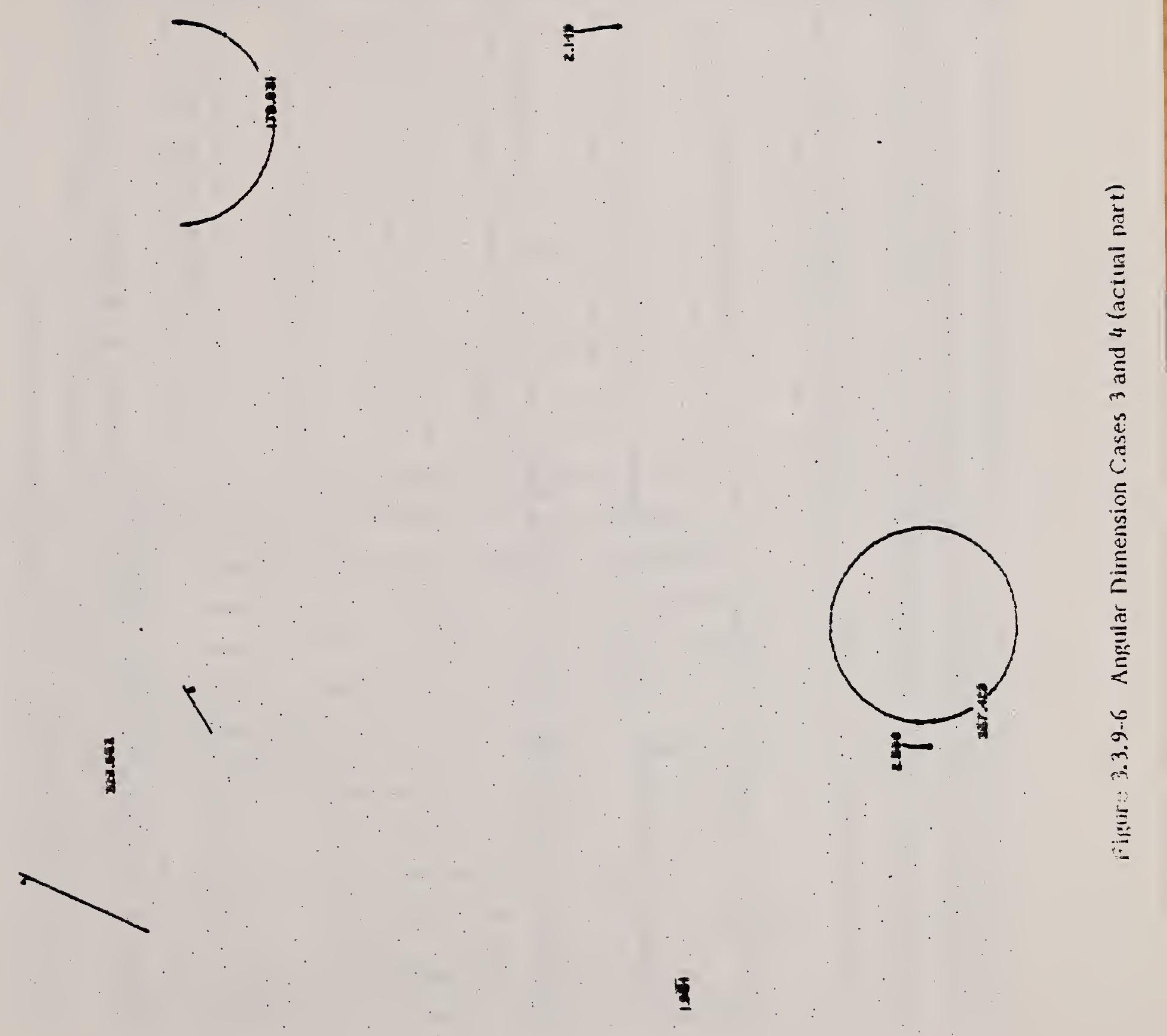




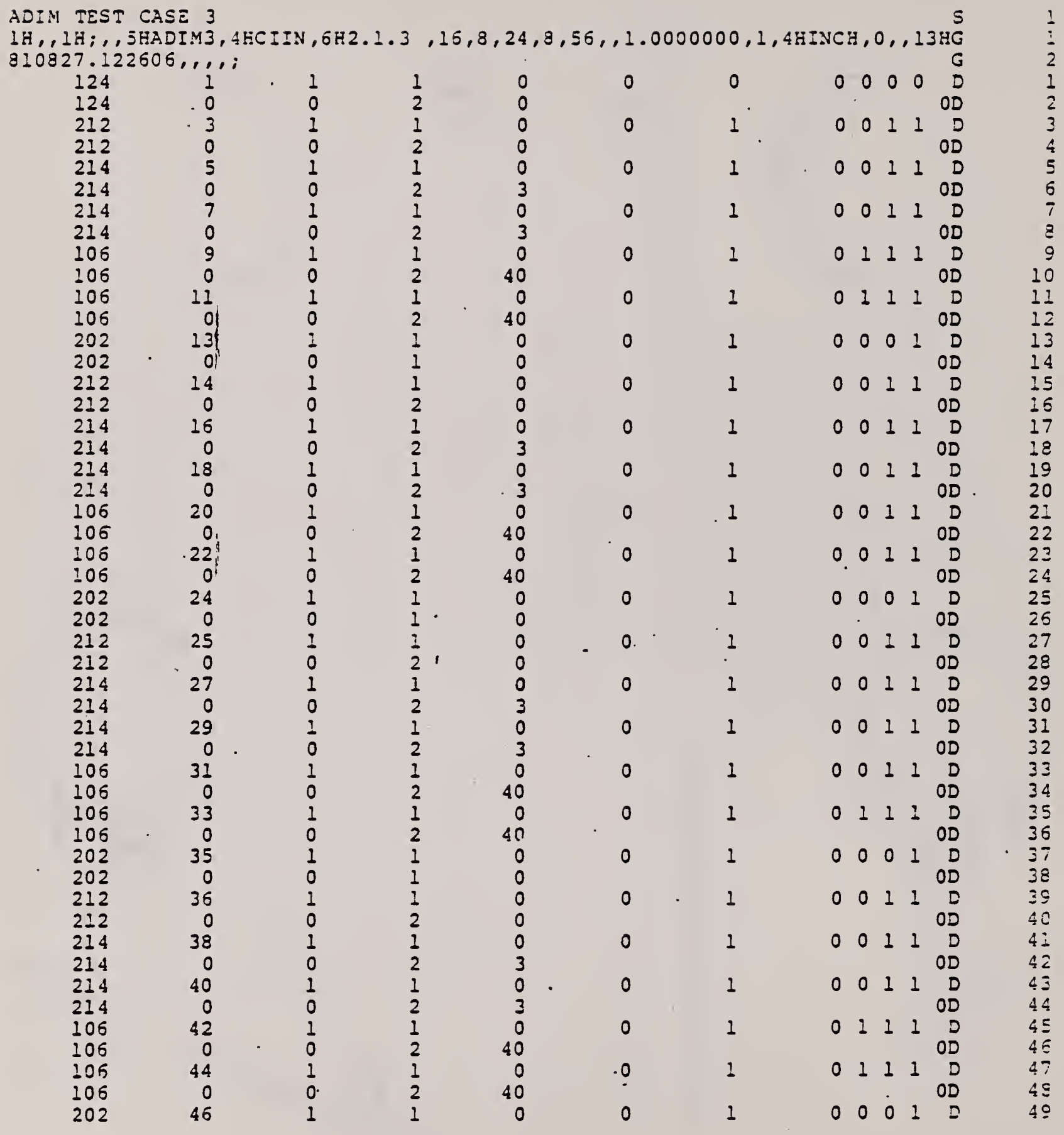




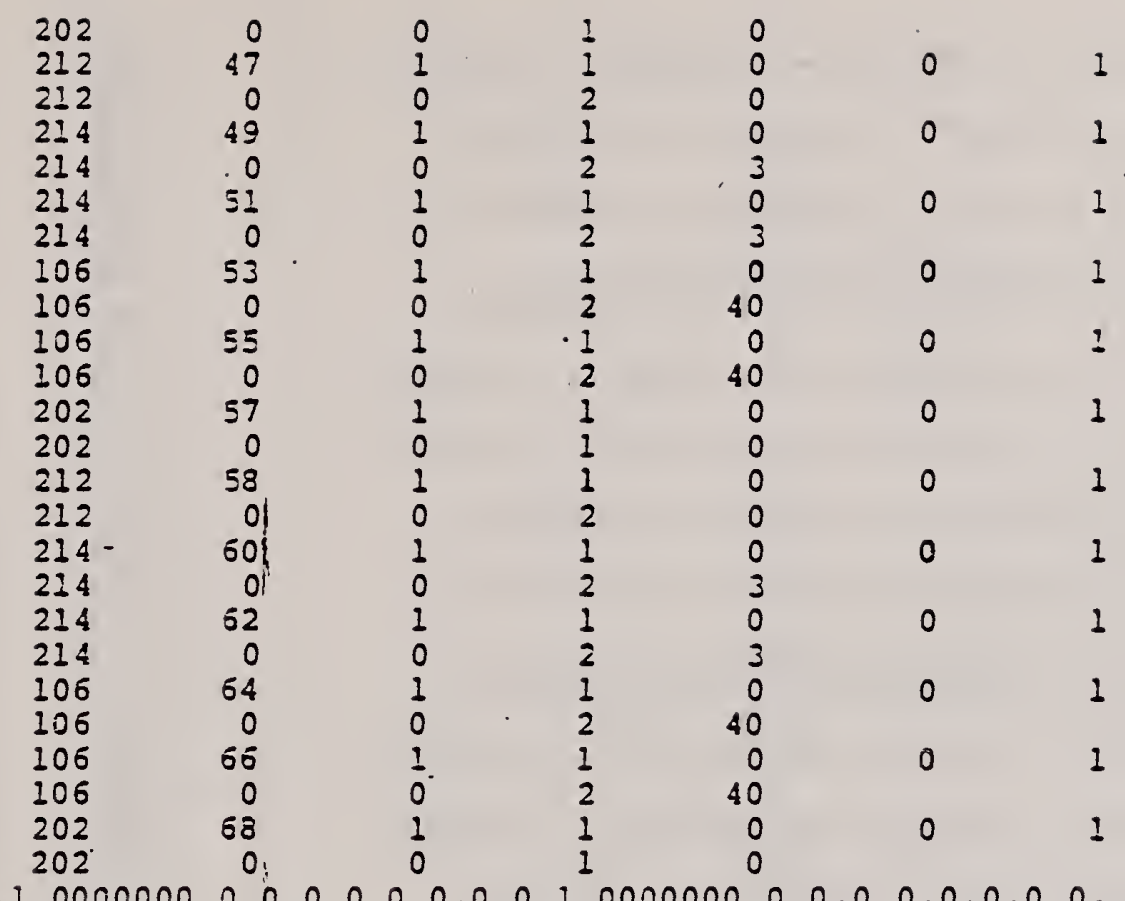

$124,1.0000000,0.0,0.0,0.0,0.0,1.0000000,0.0,0.0,0.0,0.0$, $1.0000000,0.0,0,0$;

$212,1,7, .7360000, .1560000,1,1.5707963,0.0,0,0,9.7659798$, $3.1625695,0.0,717179.831,0,0$;

$214,1, .1500000, .0500000,0.0,7.9445788,5.0871625,9.9548096$, $3.1922900,0,0$;

$214,1, .1500000, .0500000,0.0,12.8118275,5.1565377,10.8410751$, $3.4475394,0,0$;

$106,1,3,0.0,4.5023680,5.0203238,4.5023680,5.0203238,7.8196024$, $5.0847359,0,0$ :

$106,1,3,0.0,16.2026584,5.2288150,16.2026584,5.2288150$, $11.9368106,5.1585951,0,0$;

$202,3,9,31,9.8781519,5.1247077,1.9339375,5,7,0,0$;

$212,1,7,1.0608000, .1560000,1,1.5707963,0.0,0,0,-3.0394285$, $6.7518706,0.0,78326.853,0,0$;

$214,1, .1500000, .0500000,0.0,-4.6677636,8.5493691,-4.6677627$, $8.5493692,0,0$;

$214,1, .1500000, .0500000,0.0,-1.0990795,5.2365504,-1.0990797$, $5.2368508,0,0$;

$106,1,3,0.0,-5.8513827,5.1529373,-5.8518827,5.1529373$,

$-4.5123899,8.66: 4350,0,0$;

$106,1,3,0.0,-1.9059879,4.7604312,-1.9059879,4.7604312,-.9914409$, $5.3004030,0,0$;

$202,15,21,23,-3.4487181, .8974361,8.5350866,17,19,0,0$;

$212,1,5, .4864000, .1560000,1,1.5707963,0.0,0,0,-7.7697778$,

$-4.4404740,0.0,5 \% 1.984,0,0$;

$214,1, .1500000, .0500000,0.0,-7.2654505,-4.2080320 ;-7.2654505$,

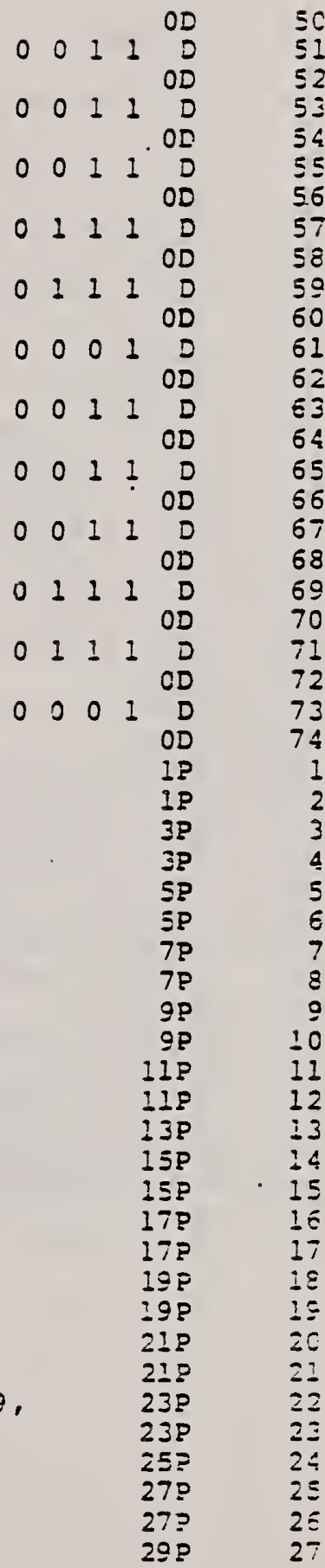


$-4.2080307,0,0$;

$214,1, .1500000, .0500000,0.0,-7.2585234,-4.4484209,-7.2585236$, $-4.4484201,0,0$;

$106,1,3,0.0,-7.1065645,-4.2062057,-7.1065645,-4.2062057$,

$-7.3904423,-4.2094687,0,0$;

$106,1,3,0.0,-7.0808301,-4.4402197,-7.0808301,-4.4402197$,

$-7.3833905,-4.4541840,0,0$;

$202,27,33,35,-.3205132,-4.1282053,6.9453959,29,31,0,0$;

$212,1,5, .6396000, .1560000,1,1.5707963,0.0,0,0,10.9063444$,

$-2.6749942,0.0,5 \pm 2.149,0,0$;

$214,1, .1500000, .0500000,0.0,11.5155231,-3.6310084,11.4372584$, $-2.7734415,0,0$;

$214,1, .1500000, .0500000,0.0,11.5101315,-3.4346487,11.5101314$, $-3.4346487,0,0$;

$106,1,3,0.0,15.2190965,-5.5988032,15.2190965,-3.5988032$,

$11.6405184,-3.6299214,0,0$;

$106,1,3,0.0,15.2446404,-3.2619545,15.2446404,-3.2619545$, $: 1.6349981,-3.4288745,0,0$;

$202,39,45,47,6.2791958,-3.6765418,5.2365255,41,43,0,0$;

$212,1,5, .7176000, .1560000,1,1.5707963 ; 0.0,0,0,-3.0077527$,

$-8.7907065,0.0,542.580,0,0$;

$214,1, .1500000, .0500000,0.0,-2.5673502,-9.2751873,-2.5573502$, $-9.2751869,0,0$;

$214,1, .1500000, .0500000,0.0,-2.5678348,-9.3828250,-2.5252591$,

$-8.8886105,0,0$;

$106,1,3,0.0,-7.3038775,-9.1471730,-7.3038775,-9.1471730$,

$-2.5923046,-0.2718101,0,0$;

$106,1,3,0.0,-7.3038965,-9.4681596,-7.3038965,-9.4681596$,

$-2.6928145,-9.3850759,0,0$;

$202,51,57,59,-.1779904,-9.3397646,2.3902323,53,55,0,0$;

$212,1,7,1.0452000, .1550000,1,1.5707963,0.0,0,0,-2.4164591$,

$-10.5645876,0.0,73357.420,0,0$;

$214,1, .1500000, .0500000,0.0,-2.0859765,-9.3741428,-1.8444463$,

$-10.2695590,0,0$

$214,1, .1500000, .0500000,0.0,-2.0855896,-9.2882078,-1.5782240$,

$-10.6362750,0,0$

$106,1,3,0.0,-7.3038955,-9.4681596,-7.3038965,-9.4681596$,

$-2.2109562,-9.3763947,0,0$;

$106,1,3,0.0,-7.3033775,-9.1471730,-7.3038775,-9.1471730$,

$-2.2105440,-9.2848307,0,0$;

$202,63,69,71,-.1779899,-9.3397646,1.9082963,65,67,0,0$;

S

16

20

$74 P$

68

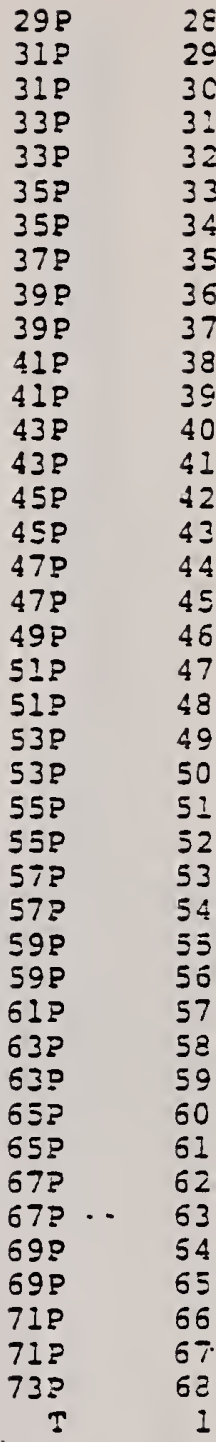




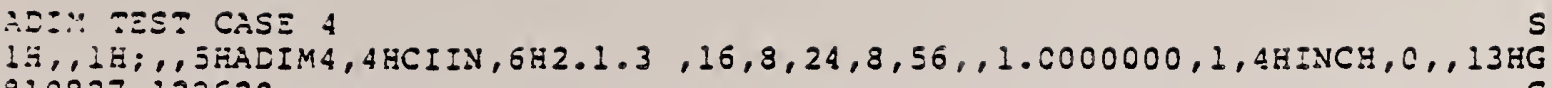
$310827.122620, \ldots$, ;

$\begin{array}{llll}124 & 1 & 1 & 1 \\ 124 & 0 & 0 & 2 \\ 212 & 3 & 1 & 1 \\ 212 & 0 & 0 & 2 \\ 214 & 5 & 1 & 1 \\ 214 & 0 & 0 & 2 \\ 214 & 7 & 1 & 1 \\ 214 & 0 & 0 & 2 \\ 106 & 9 & 1 & 1 \\ 106 & 0 & 0 & 2 \\ 106 & 11 & 1 & 1 \\ 106 & 0 & 0 & 2 \\ 202 & 13 & 1 & 1 \\ 202 & 0 & 0 & 1 \\ 212 & 24 & 1 & 1 \\ 212 & 0 & 0 & 2 \\ 214 & 16 & 1 & 1 \\ 214 & 0 & 0 & 2 \\ 214 & 18 & 1 & 1 \\ 214 & 0 & 0 & 2 \\ 105 & 20 & 1 & 1 \\ 106 & 0 & 0 & 2 \\ 106 & 22 & 1 & 1 \\ 106 & 0 & 0 & 2 \\ 202 & 24 & 1 & 1 \\ 202 & 0 & 0 & 1 \\ 212 & 25 & 1 & 1 \\ 212 & 0 & 0 & 2 \\ 214 & 27 & 1 & 1 \\ 214 & 0 & 0 & 2 \\ 214 & 29 & 1 & 1 \\ 214 & 0 & 0 & 2 \\ 105 & 31 & 1 & 1 \\ 106 & 0 & 0 & 2 \\ 106 & 33 & 1 & 1 \\ 105 & 0 & 0 & 2 \\ 202 & 35 & 1 & 1 \\ 202 & 0 & 0 & 1 \\ 212 & 36 & 1 & 1 \\ 212 & 0 & 0 & 2 \\ 214 & 38 & 1 & 1 \\ 214 & 0 & 0 & 2 \\ 214 & 40 & 1 & 1 \\ 214 & 0 & 0 & 2 \\ 106 & 42 & 1 & 1 \\ 105 & 0 & 0 & 2 \\ 106 & 44 & 1 & 1 \\ 120 & 0 & 0 & 2 \\ 202 & 46 & 1 & 1 \\ & & & \end{array}$

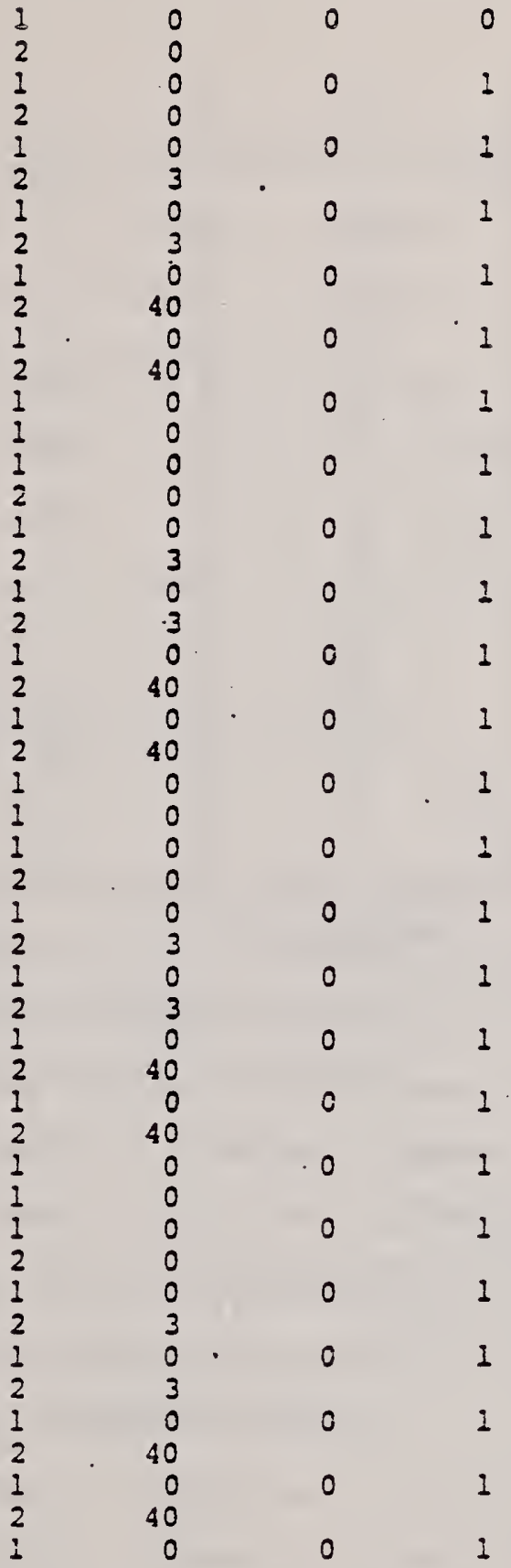

$\begin{array}{lllll}0 & 0 & 0 & 0 & 0\end{array}$

$\begin{array}{llllll}0 & 0 & 1 & 1 & 0\end{array}$

$\begin{array}{llllll}0 & 0 & 1 & 1 & 0\end{array}$

$\begin{array}{lllllllll}0 & 0 & 1 & 1 & 0 & 0\end{array}$

$0,1,10 D$

$\begin{array}{lllll}0 & 1 & -1 & 0 D \\ 0 & 1 & 1 & 0 & 0\end{array}$

$\begin{array}{lllll}0 & 1 & 1 & 1 & 0\end{array}$

$\begin{array}{lllllll}0 & 0 & 0 & 1 & 0\end{array}$

$\begin{array}{lllllll}0 & 0 & 1 & 1 & 0\end{array}$

$\begin{array}{llll}0 & 0 & 1 & 0 D \\ 0\end{array}$

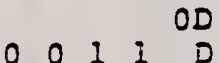

19

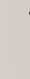

$0,1, O D$

- 20

$\begin{array}{llllll}0 & 0 & 1 & 1 & D & 21\end{array}$

$\begin{array}{lllllll}0 & 0 & 1 & 1 & 0 D & 22\end{array}$

OD 24

$\begin{array}{llllll}0 & 0 & 0 & 1 & D & 25\end{array}$

$\begin{array}{lllll}0 & 0 & 1 & 0 D & 26 \\ 0 & 27\end{array}$

$\begin{array}{llllll}0 & 0 & 1 & 1 & D & 29\end{array}$

O 30

32

$\begin{array}{lllllllll}0 & 0 & 1 & 1 & D & 3\end{array}$

$0111 D$ 3S

$\begin{array}{llllllll}0 & 0 & 0 & 1 & 0 & 36\end{array}$

$\begin{array}{llllll}0 & 0 & 1 & 1 & 0 & 0\end{array}$

$00110 D$ OD

0.150242

$O D \quad 44$

$\begin{array}{llllll}0 & 1 & 1 & 1 & 0 & 45\end{array}$

$0: 110$ 4.

$\begin{array}{llllll}0 & 0 & 0 & 1 & 0 & 4 \varepsilon\end{array}$ 


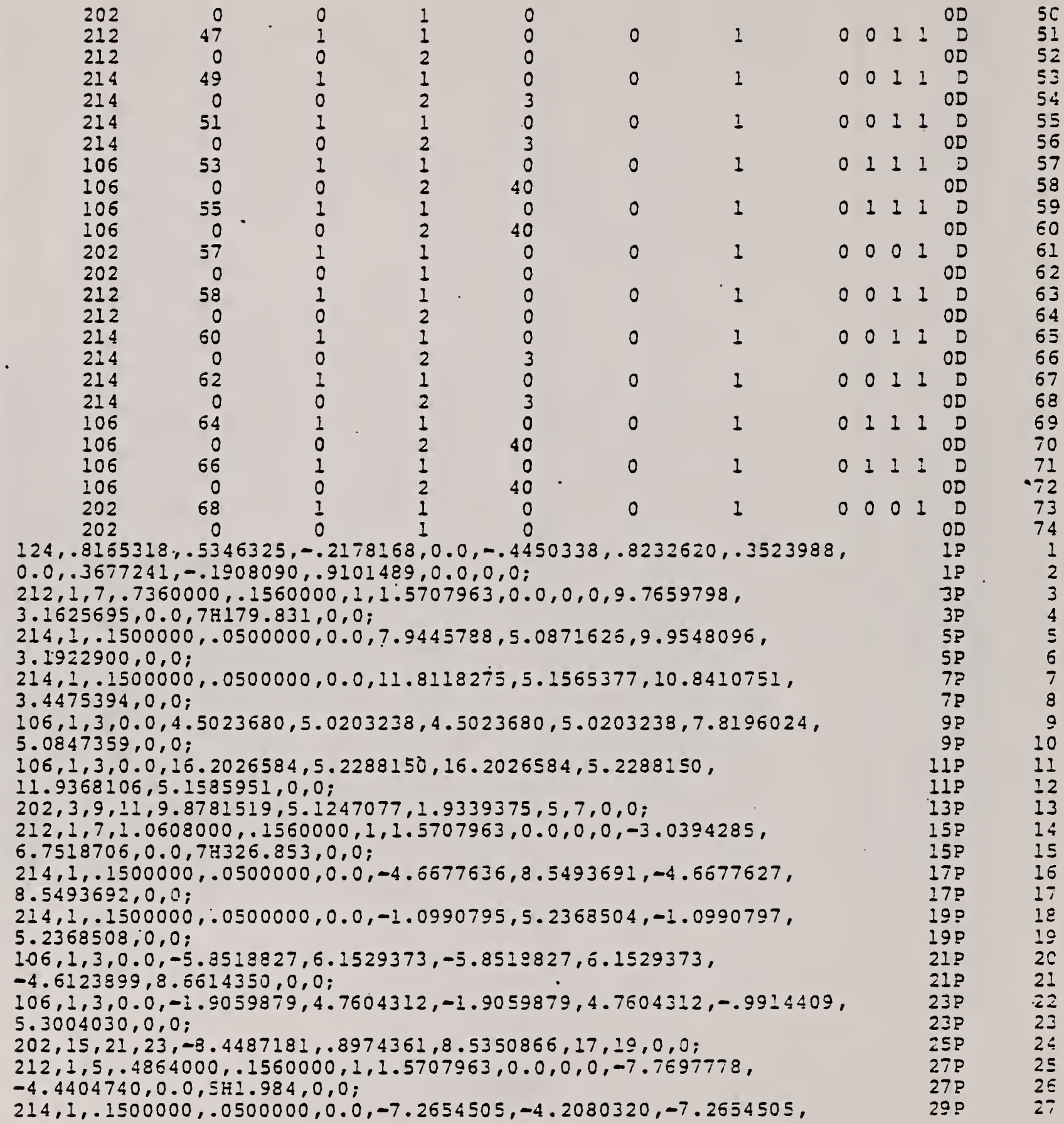


$-4.2080307,0,0$;

$214,1, .1500000, .0500000,0.0,-7.2585234,-4.4484209,-7.2585236$, $-4.4484201,0,0$;

$106,1,3,0.0,-7.1065645,-4.2062057,-7.1065645,-4.2062057$,

$-7.3904423,-4.2094687,0,0$;

$106,1,3,0.0,-7.0808301,-4.4402197,-7.0808301,-4.4402197$,

$-7.3833905,-4.4541840,0,0$;

$202,27,33,35,-.3205132,-4.1282053,6.9453959,29,31,0,0$;

$212,1,5, .6396000, .1560000,1,1.5707963,0.0,0,0,10.9063444$,

$-2.6749942,0.0,5 \mathrm{H} 2.149,0,0$;

$214,1, .1500000, .0500000,0.0,11.5155231,-3.6310084,11.4372584$,

$-2.7734415,0,0$;

$214,1, .1500000, .0500000,0.0,11.5101315,-3.4346487,11.5101314$,

$-3.4346487,0,0$;

$106,1,3,0.0,15.2190965,-3.5988032,15.2190965,-3.5988032$,

$11.6405134,-3.5299214,0,0$;

$106,1,3,0.0,15.2446404,-3.2619545,15.2446404,-3.2619545$,

$11.6349981,-3.4288745,0,0$;

$202,39,45,47,6.2791958,-3.6765418,5.2365255,41,43,0,0$;

$212,1,5, .7175000, .1560000,1,1: 5707963,0.0,0,0,-3.0077527$,

$-8.7907066,0.0,5 \mathrm{H} 2.580,0,0$;

$214,1, .1500000, .0500000,0.0,-2.5673502,-9.2752873,-2.5673502$,

$-9.2751869,0,0$;

$214,1, .1500000, .0500000,0.0,-2.5678348,-9.3828250,-2.5252591$, $-8.8886105,0,0$

$106,1,3,0.0,-7.3038775,-9.1471730,-7.3038775,-9.1471730$,

$-2.6923046,-9.2718101,0.0$;

$106,1,3,0.0,-7.3038965,-9.4681596,-7.3038965,-9.4681596$,

$-2.6928145,-9.3850769,0,0$

$202 ، 51,57,59,-.1779904,-9.3397646,2.3902323,53,55,0,0$;

$212,1,7,1.0452000, .1560000,1,1.5707963,0.0,0,0,-2.4164591$,

$-10.5545876,0.0,74357,420,0,0$;

$214,1, .1500000, .0500000,0.0,-2.0859755,-9.3741428,-1.8444463$,

$-10.2695590,0,0$;

$214,1, .1500000, .0500000,0.0,-2.0855896,-9.2882078,-1.5782240$,

$-10.6362750,0,0$;

$106,1,3,0.0,-7.3038965,-9.4681596,-7.3038965,-9.4681596$,

$-2.2109562,-9.3763947,0,0$;

$106,1,3,0.0,-7.3038775,-9.1471730,-7.3038775,-9.1471730$,

$-2.2105440,-9.2848307,0,0$;

$202,63,69,71,-.1779899,-9.3397546,1.9082963,55,67,0,0$;

$\mathrm{S}$

$1 G \quad 20$

$74 P$

58

$\begin{array}{ll}29 P & 28 \\ 31 P & 29 \\ 31 P & 30 \\ 33 P & 31 \\ 33 P & 32 \\ 35 P & 33 \\ 35 P & 34 \\ 37 P & 35 \\ 39 P & 36 \\ 39 P & 37 \\ 41 P & 38 \\ 41 P & 39 \\ 43 P & 40 \\ 43 P & 41 \\ 45 P & 42 \\ 45 P & 43 \\ 47 P & 44 \\ 47 P & 45 \\ 49 P & 45 \\ 51 P & 47 \\ 51 P & 48 \\ 53 P & 49 \\ 53 P & 50 \\ 55 P & 51 \\ 55 P & 52 \\ 57 P & 53 \\ 57 P & 54 \\ 59 P & 55 \\ 59 P & 56 \\ 61 P & 57 \\ 63 P & 58 \\ 63 P & 59 \\ 55 P & 60 \\ 65 P & 61 \\ 67 P & 62 \\ 67 P & 63 \\ 59 P & 64 \\ 69 P & 55 \\ 71 P & 56 \\ 71 P & 67 \\ 73 P & 68 \\ T & 1\end{array}$





\subsubsection{Diameter Dimension}

The diameter dimensions contain subentities which include leaders, arc center points and general notes.

The following conventions were followed:

- general note subentities to be single string with no box rotation

- leaders to have one, two, or three segments

Case 1 (Figures $3 \cdot 3 \cdot 10-1$ and 3.3.10-2) tests the following:

- text in, arrows in configuration

- text out, arrows out configuration

- singie segment leaders

- multiple segment leaders

Case 2

- This is identical to Case 1 except non-model space with rotation $\left(-25.86^{\circ}, 158.76^{\circ}, 33.48^{\circ}\right)$ is used per section 3.0 

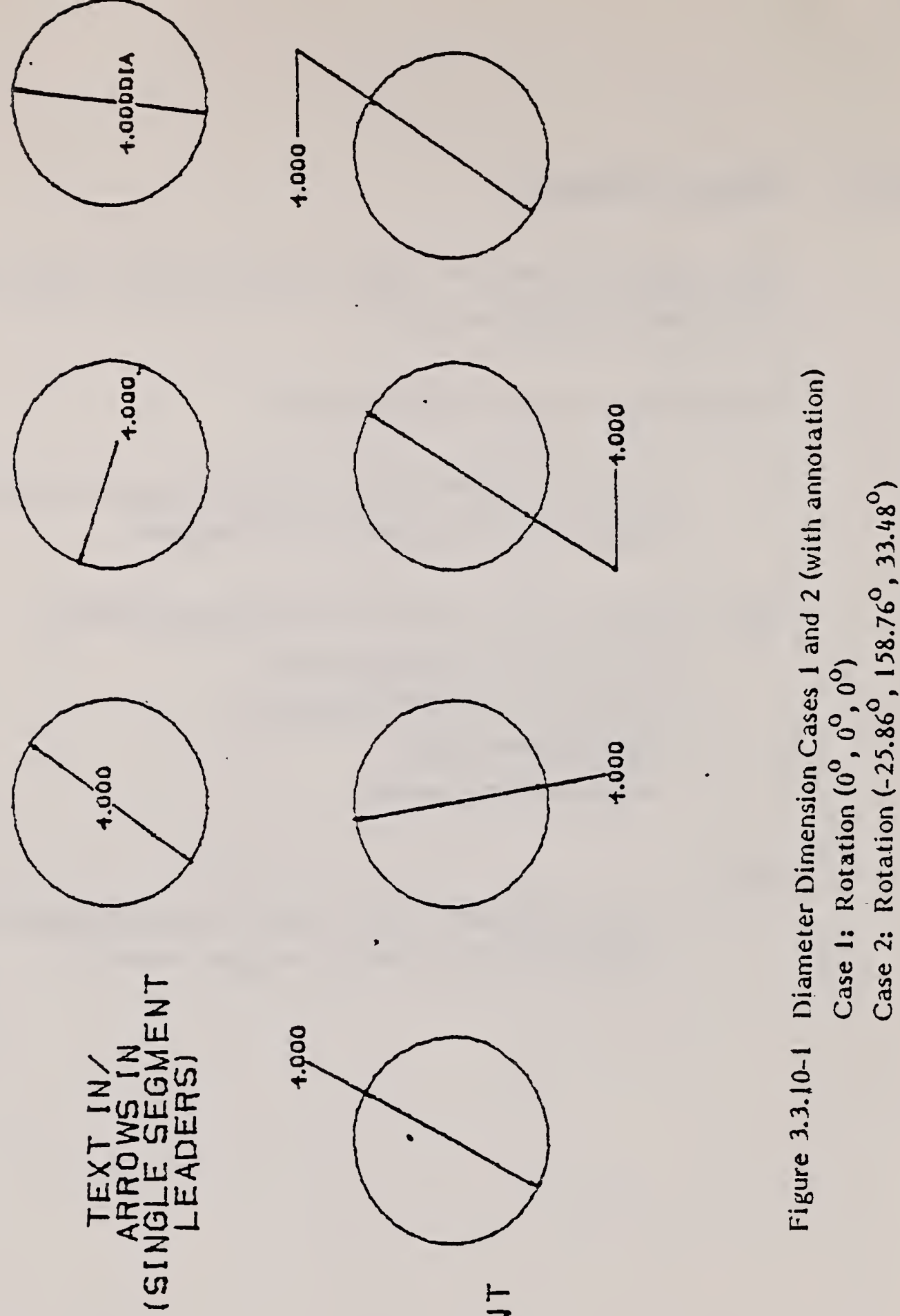

$\frac{1}{0}$

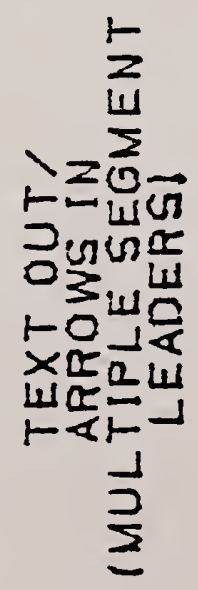

$3.10-2$ 

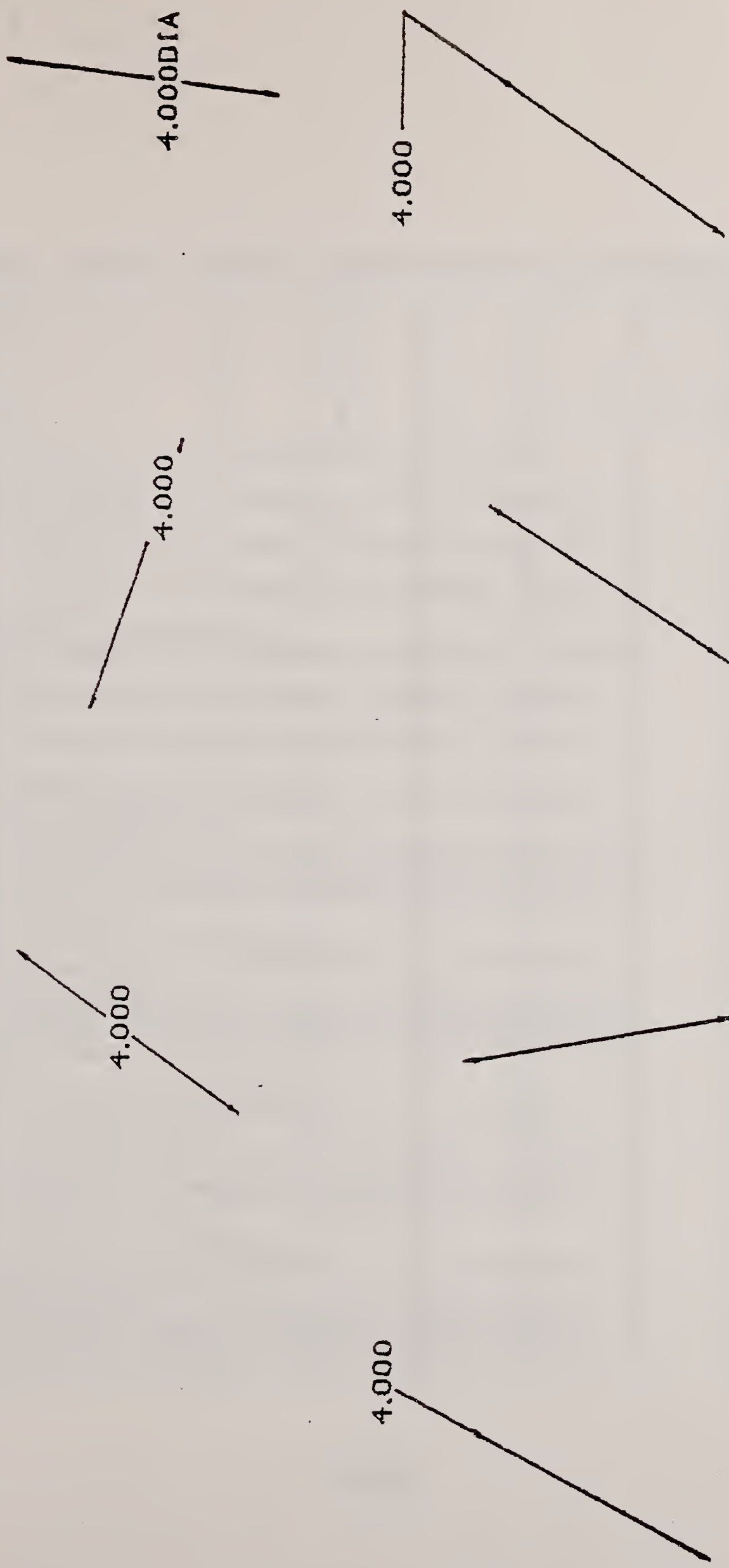

$3.10-3$ 


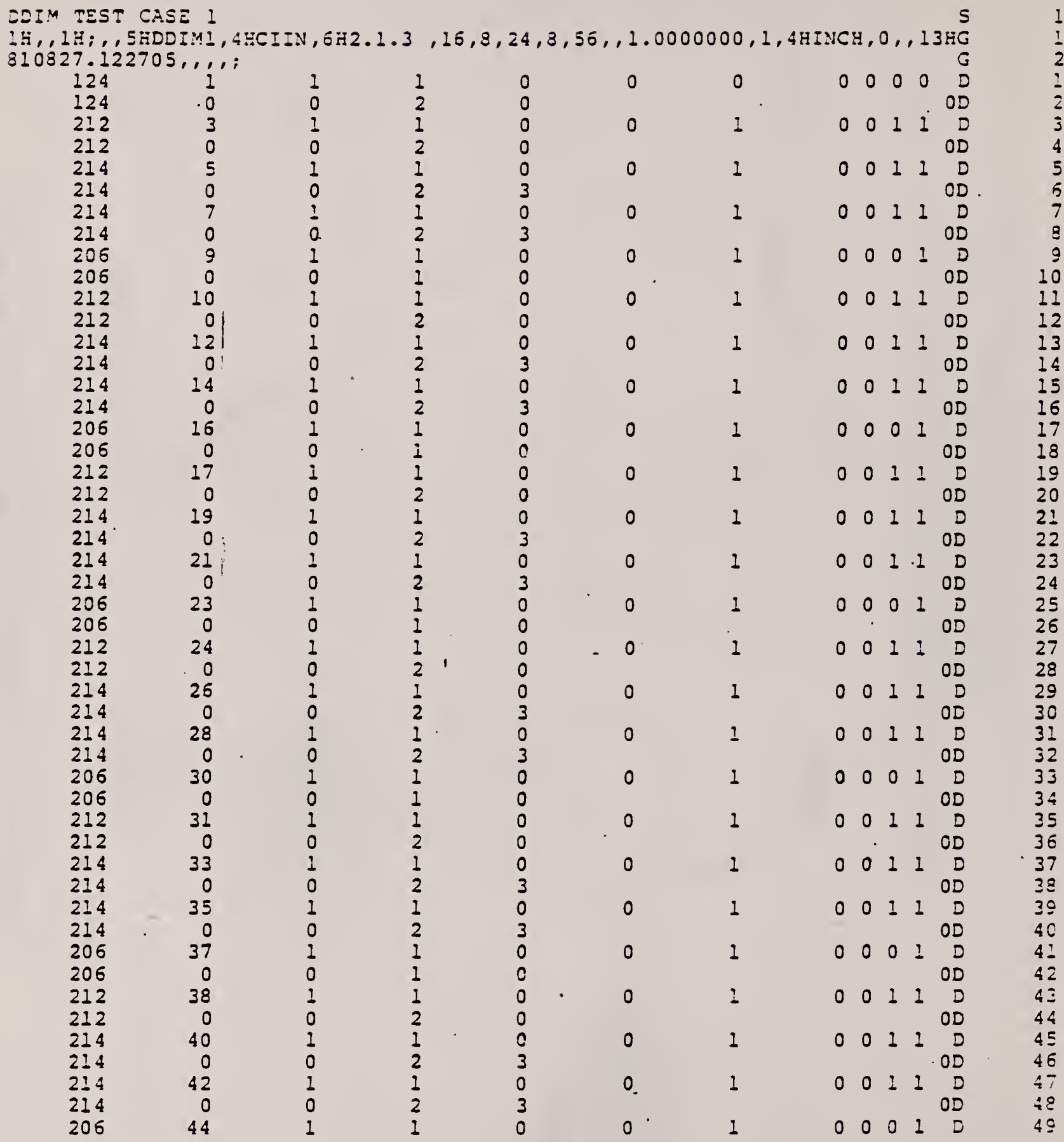




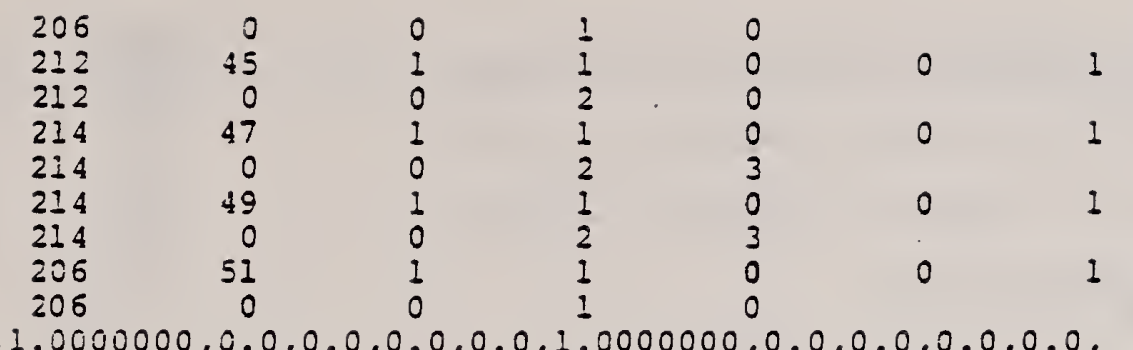

$124,1.0000000,0.0,0.0,0.0,0.0,1.0000000,0.0,0.0,0.0,0.0$, i. $0000000,0.0,0,0$;

$=: \therefore,=, \therefore: 00000, .2500000,1,1.5707963,0.0,0,0,-7.5000000$,

$2.5000000,0.0,5 \mathrm{H} 4.000,0,0$;

$214,1, .1500000, .0500000,0.0,-8.1236267, .8454127,-7.0578113$,

$2.4000002,0,0$;

$214,1, .1500000, .0500000,0.0,-5.8762851,4.1544089,-6.7621890$,

$2.8499999,0,0$;

$206,3,5,7,-5.9999559,2.4999108,0,0$;

$212,1,5,1.1500000, .2500000,1,1.5707963,0.0,0,0,0.0,2.0000000$, $0.0,5 \mathrm{~B} \div .000,0,0$;

$214,1, .1500000, .0500000,0.0,-2.3903754,3.1530671,-.0861823$, $2.3499999,0,0$;

$214,1, .2500000, .0500000,0.0,1.3903035,1.8467474,1.2361828$, 1. $9000000,0,0$;

$206,11,13,15,-.5000359,2.4999073,0,0$;

$212,1,5,1.1500000, .2500000,1,1.5707963,0.0,0,0,-12.5000000$, - $.5000000,0.0,5 \mathrm{H} 4.000,0,0$;

$2: 4,3, .5500000, .0500000,0.0,-13.3404798,-2.5064119,-13.4999123$,

$-4.5000467,-13.2500000,-1.3750000,-12.7500000,-1.3750000,0,0$;

$214,1, .1500000, .05500000,0.0,-13.6593447,-6.4936816,-13.4999123$,

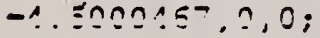

$206,13,21,23,-13.4999123,-4.5000467,0,0$;

$212, \pm, 5, \pm .1500000, .2500000,1,1.5707963,0.0,0,0,-7.0000000$,

$-8.0000000,0.0,544.000,0,0$;

$214,3, .1500000, .0500000,0.0,-7.4339069,-6.4524032,-7.0000973$,

$-4.5000176,-7.7500000,-7.8750000,-7.2500000,-7.8750000,0,0$;

$214,1, .1500000, .0500000,0.0,-5.5662886,-2.5476321,-7.0000978$, $-4.5000175,0,0$;

$206,27,29,31,-7.0000978,-4.5000176,0,0$;

$212,1,5,1.1500000, .2500000,1,1.5707963,0.0,0,0,-.5000000$,

$-3.0000000,0.0,544.000,0,0$;

$214,3, .1500000, .0550000,0.0,-1.5195628,-5.2205030,-.4999143$,

$-4.5000505,-2.5000000,-7.8750000,-.6000000,-7.8750000,0,0$;

$214,1, .1500000, .0500000 ; 0.0, .5197343,-2.7794931,-.4999143$, $-4.5000505,0,0$;

$206,35,37,39,-.4999143,-4.5000505,0,0$;

$2 \$ 2,1,5,1.1500000, .2500000,1,1.5707963,0.0,0,0,4.5000000$,

$-i .5000000,0.0,5 H 4.000,0,0$;

$214,3, .1500000, .0500000,0.0,6.5780690,-2.8154324,5.4999166$, $-4,15 \equiv \vdots \equiv \equiv, 7.5000000,-1.3750000,5.8499999,-1.37 \equiv 0000,0,0$;

$214,1, .1500000, .0500000,0.0,4.42 i 7641,-5.1844602,5.4999166$,

$-4 . \$ 999466,0,0$; 
$206,43,45,47,5.4999166,-4.4999466,0,0$;

$212,1,8,1.9250000, .2500000,1,1.5707963,0.0,0,0,5.5000000$, 2.0000000,0.0,8H4.000DIA, 0,0;

$214,1, .1500000, .0500000,0.0,6.7115493,4.4887428,6.4839445$, $2.3500000,0,0$;

$214,1, .1500000, .0500000,0.0,6.2882500, .5112029,6.4360556$, $1.9000000,0,0$;

$205,51,53,55,6.4999046,2.4999729,0,0$;

$\mathrm{S}$

$2 D$

$58 ?$

51

$\begin{array}{rr}49 P & 41 \\ 513 & 45 \\ 519 & 46 \\ 532 & 47 \\ 539 & 48 \\ 539 & 49 \\ 559 & 50 \\ 57 ? & 51 \\ 1 & 1\end{array}$


DDIM TEST CASE 2

IH, $1 \mathrm{H} ;,, 5 \mathrm{HDDIM} 2,4 \mathrm{HCIIN}, 6 \mathrm{H} 2.1 .3,16,8,24,8,56,1.0000000,1,4 \mathrm{HINCH}, 0,1,3 \mathrm{HG}$

$810827.122719, \ldots$;

$\begin{array}{lrll}124 & 1 & 1 & 1 \\ 124 & 0 & 0 & 2 \\ 212 & 3 & 1 & 1 \\ 212 & 0 & 0 & 2 \\ 214 & 5 & 1 & 1 \\ 214 & 0 & 0 & 2 \\ 214 & 7 & 1 & 1 \\ 214 & 0 & 0 & 2 \\ 200 & 0 & 1 & 1 \\ 206 & 0 & 0 & 1 \\ 212 & 10 & 1 & 1 \\ 212 & 0 & 0 & 2 \\ 214 & 12 & 1 & 1 \\ 214 & 0 & 0 & 2 \\ 214 & 14 & 1 & 1 \\ 214 & 0 & 0 & 2 \\ 206 & 16 & 1 & 1 \\ 206 & 0 & 0 & 1 \\ 212 & 17 & 1 & 1 \\ 212 & 0 & 0 & 2 \\ 214 & 19 & 1 & 1 \\ 214 & 0 & 0 & 2 \\ 214 & 21 & 1 & 1 \\ 214 & 0 & 0 & 2 \\ 206 & 23 & 1 & 1 \\ 206 & 0 & 0 & 1 \\ 212 & 24 & 1 & 1 \\ 212 & 0 & 0 & 2 \\ 214 & 26 & 1 & 1 \\ 214 & 0 & 0 & 2 \\ 214 & 23 & 1 & 1 \\ 214 & 0 & 0 & 2 \\ 206 & 30 & 1 & 1 \\ 206 & 0 & 0 & 1 \\ 212 & 31 & 1 & 1 \\ 212 & 0 & 0 & 2 \\ 214 & 33 & 1 & 1 \\ 214 & 0 & 0 & 2 \\ 214 & 35 & 1 & 1 \\ 214 & 0 & 0 & 2 \\ 205 & 37 & 1 & 1 \\ 206 & 0 & 0 & 1 \\ 212 & 38 & 1 & 1 \\ 212 & 0 & 0 & 2 \\ 214 & 40 & 1 & 1 \\ 214 & 0 & 0 & 2 \\ 211 & 42 & 1 & 1 \\ 214 & 0 & 0 & 2 \\ 205 & 44 & 1 & 1 \\ & & & \end{array}$

$\begin{array}{ll}1 & 0 \\ 2 & \\ 1 & 0 \\ 2 & 0 \\ 1 & 0 \\ 2 & 0 \\ 1 & 3 \\ 2 & 0 \\ 1 & 3 \\ 1 & 0 \\ 1 & 0 \\ 2 & 0 \\ 1 & 0 \\ 2 & 3 \\ 1 & 0 \\ 2 & 3 \\ 1 & 0 \\ 1 & 0 \\ 1 & 0 \\ 2 & 0 \\ 1 & 0 \\ 2 & 3 \\ 1 & 0 \\ 2 & 3 \\ 1 & 0 \\ 1 & 0 \\ 1 & 0 \\ 2 & 0 \\ 1 & 0 \\ 2 & 3 \\ 1 & 0 \\ 2 & 3 \\ 1 & 0 \\ 1 & 0 \\ 1 & 0 \\ 2 & 0 \\ 1 & 0 \\ 2 & 3 \\ 1 & 0 \\ 2 & 3 \\ 1 & 0 \\ 1 & 0 \\ 1 & 0 \\ 2 & 0 \\ 1 & 0 \\ 2 & 3 \\ 1 & 0 \\ 2 & 3 \\ 1 & 0 \\ & \end{array}$

0
0
0
0
0
3
0
3
0
0
0
0
0
3
0
3
0
0
0
0
0
3
0
3
0
0
0
0
0
3
0
3
0
0
0
0
0
3
0
3
0
0
0
0
0
3
0
3
0

0
0
0
0
0

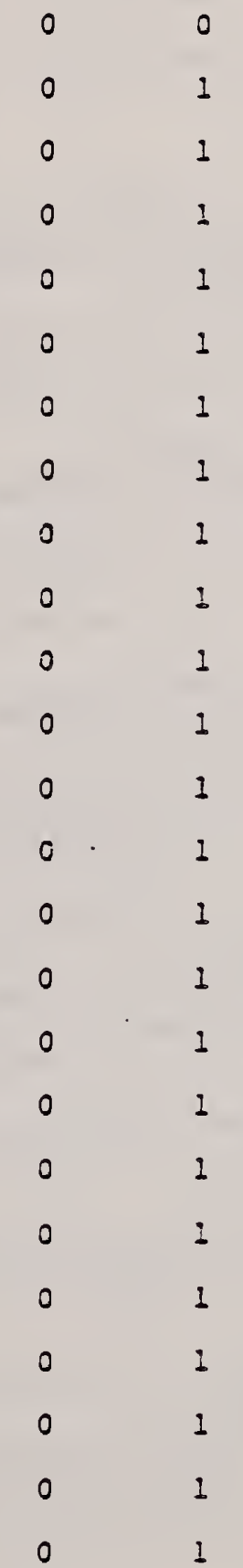

$\begin{array}{lllll}0 & 0 & 0 & 0 & 0 \\ 0\end{array}$

$\begin{array}{llllll}0 & 0 & 1 & 1 & D \\ D\end{array}$

00110

$001 ; 00$

$0,0,0 D$

$\begin{array}{llll}0 & 0 & 1 & 0\end{array}$

00110

$0 \begin{array}{lllll}0 & 0 & 1 & 1 & D\end{array}$ 


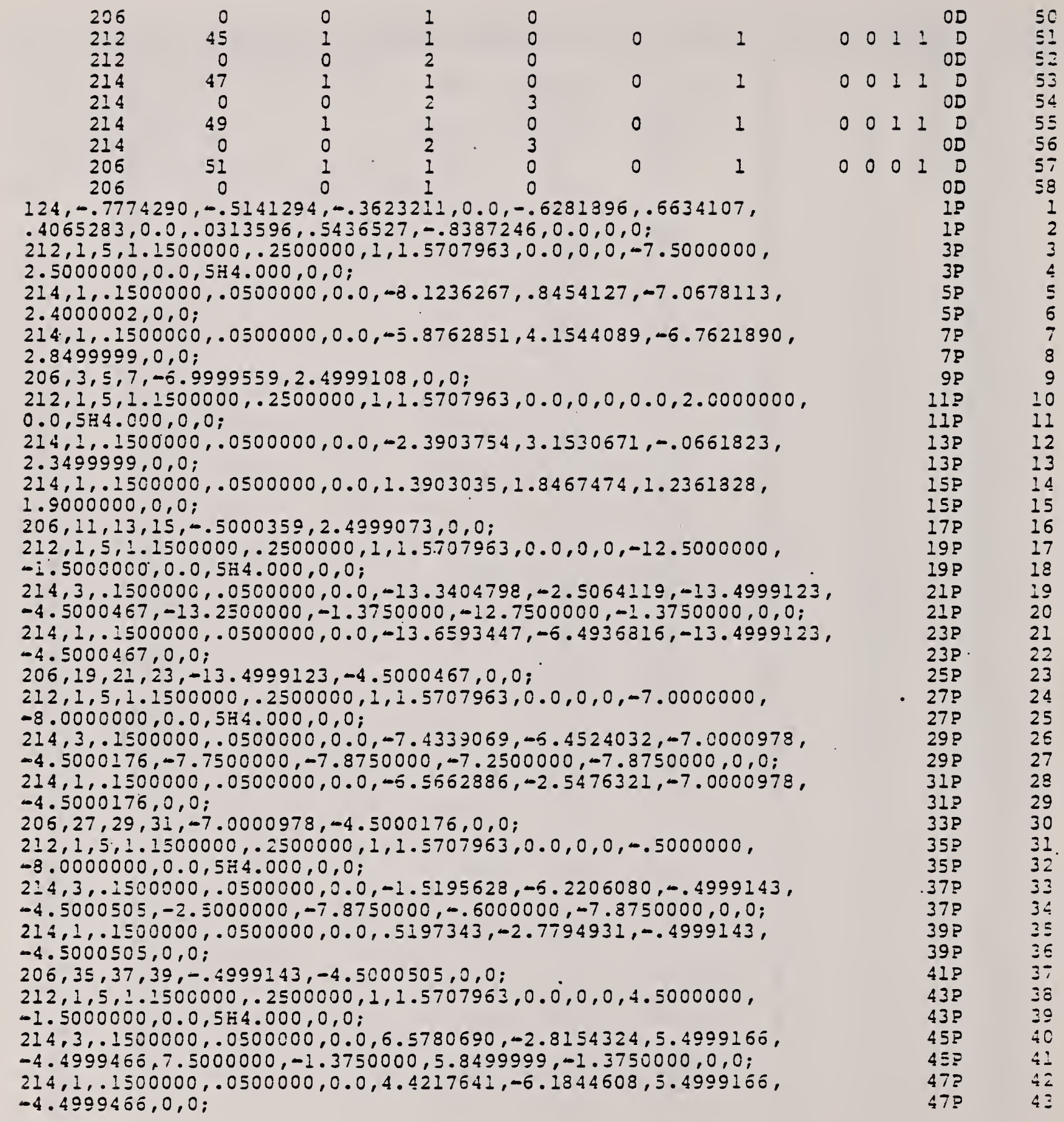

\section{$3.10-8$}


$206,43,45,47,5.4999 ! 66,-4.4999466,0,0 ;$

$212,1,8,1.9250000, .2500000,1,1.5707963,0.0,0,0,5.5000000$, $2.0000000,0.0,8 \% 4.0000 I A, 0,0$;

$214,1, .1500000, .0500000,0.0,6.7115493,4.4887428,6.4839445$, $2.3500000,0,0$ :

$214,1 . .500000,0500000,0.0,6.2882600, .5112029,6.4360556$, $1.9000000,0,0$;

$205,51,5 \pm, 55,6.4999046,2.4999729,0,0$;

5

16

20

$58 P$

51

$\begin{array}{rr}49 P & 44 \\ 51 P & 45 \\ 51 P & 46 \\ 53 P & 47 \\ 53 P & 48 \\ 55 P & 49 \\ 55 P & 50 \\ 57 P & 51 \\ T & 1\end{array}$





\subsubsection{General Label}

The general labels contain subentities which include leaders and general notes.

Case 1 (Figures 3.3.11-1 and 3.3.11-2) tests the following:

- leader from head oi text

- leader from tail oi text

- single leader labels

- multiple leader labels

- leaders with variable horizontal segments

Case 2

- This is identical to Case 1 except non-model space with rotation $\left(45^{\circ}, 99^{\circ}, 1^{\circ}\right)$ is used per section 3.0

Case 3 (Figures 3.3.11-3 and 3.3.11-4) tests the following:

- rotated labels with leader from head of text

- rotated labels with leader from tail of text

- rotated labels with multiple leaders

- rotated labels with variable horizontal segments

Case 4

- This is identical to Case 3 except non-model space with rotation $\left(90^{\circ}, 33^{\circ}, 100^{\circ}\right)$ is used per section 3.0 

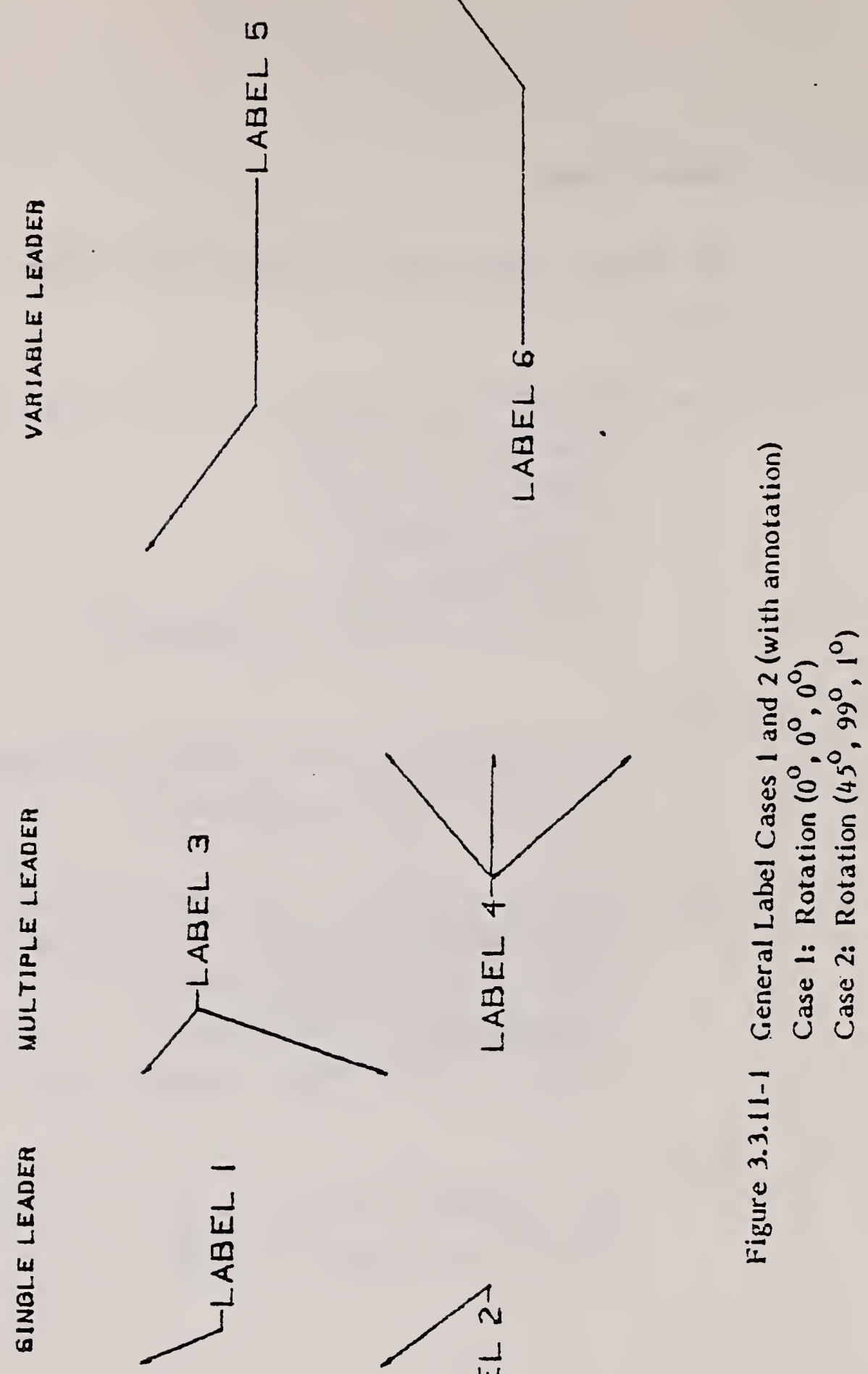

晟
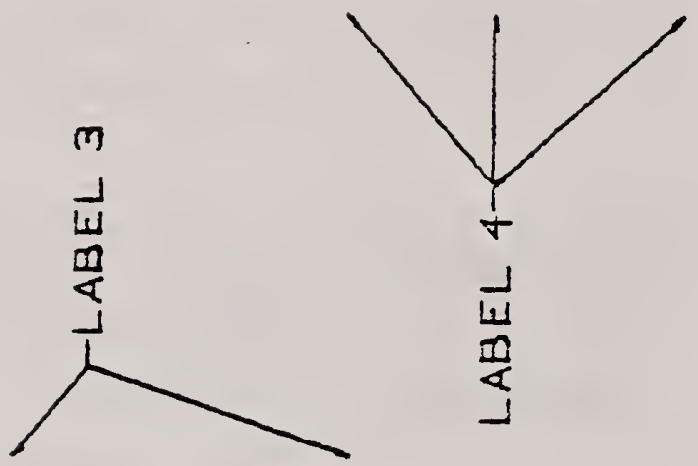

$\frac{1}{11}$

mi
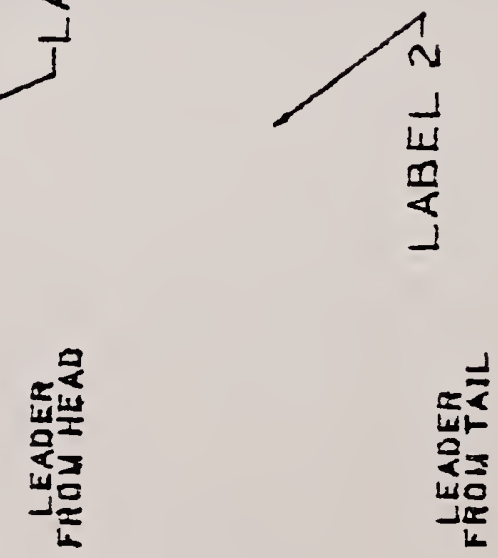

$3.11-2$ 


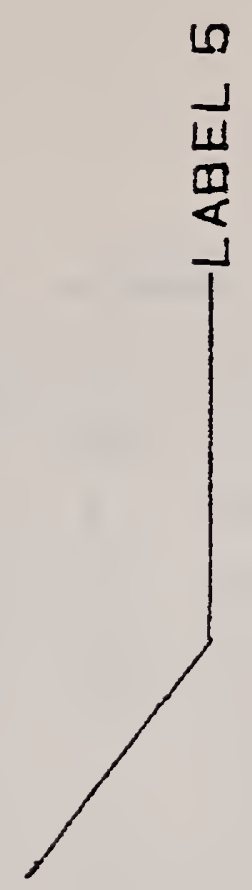


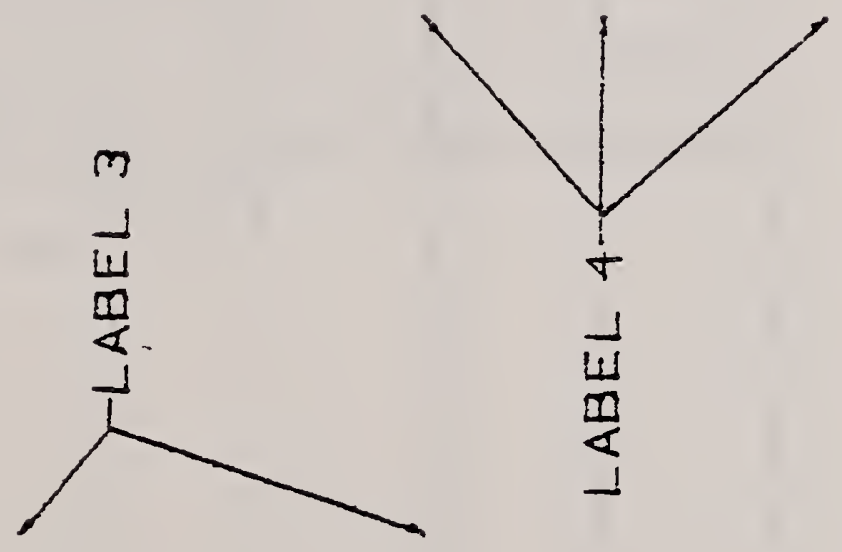

茫
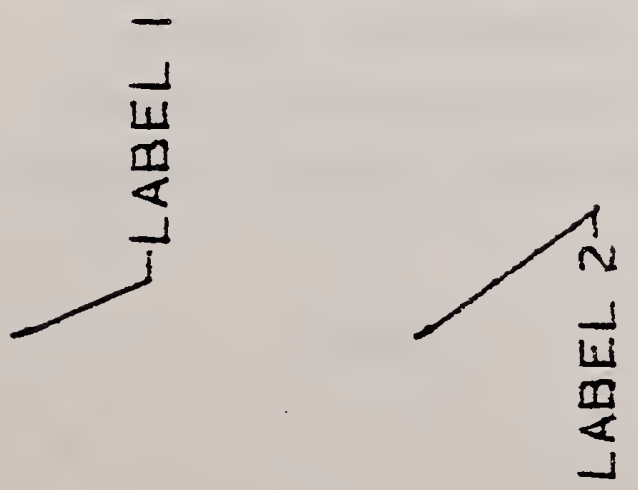

$3.11-3$ 
LAEEL CASE 1

IH, , IH; , 6 6LABEL1, 4HCIIN, 6H2.1.3, 16,8,24,8,56, 1.0000000, 1, 4HINCH, 0, , 13G :810827.122837, , ; :

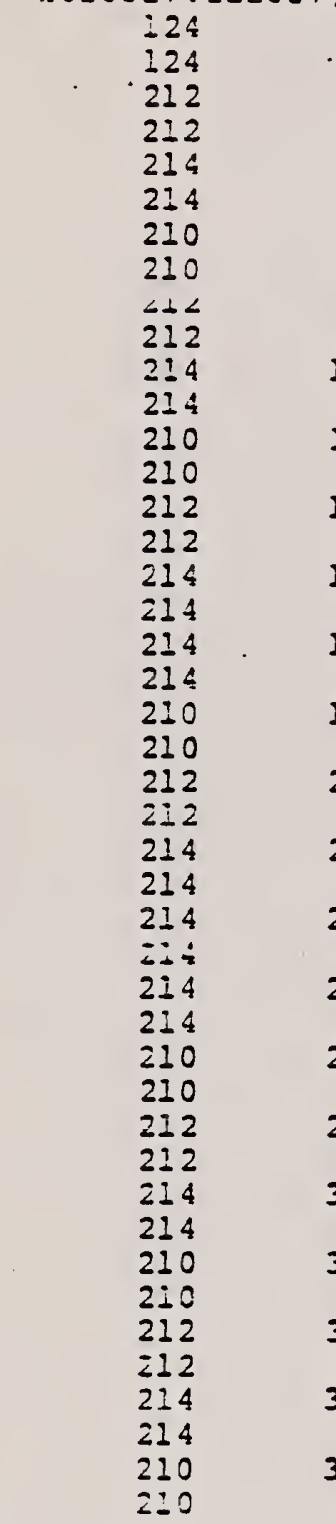

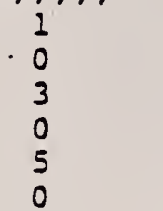

1
0
1
0
1
0
1
0
1
0
1
0
1

1

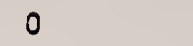

$0 \quad 0$

$\begin{array}{lllll}0 & 0 & 0 & 0 & 0\end{array}$

$$
\text { 西 }
$$

$124,1.0000000,0.0,0.0,0.0,0.0,1.0000000,0.0,0.0,0.0,0.0$, $1.0000000,0.0,0,0$;

Z: $-1.175 C C 01,0.0,7$ GLABEL 2,0,0;

$714,2.1500000,00500000,0.0,-9.0000000,1.0000000,-7.5999999$,

\section{$3.11-4$}


$-1.0000000,-7.9000001,-1.0000000,0,0$;

$2: 0,3,1,5,0,0 ;$

$212,1,7,2.2800002, .3500000,1,1.5707963,0.0,0,0,-8.0000000$,

$3.3250000,0.0,7$ KABEL $1,0,0$;

$214,2, .1500000, .0500000,0.0,-9.0000000,5.5000000,-8.3999996$,

$4.0000000,-8.1000004,4.0000000,0,0$;

$2: 0,9,1,11,0,0$;

$212,1,7,2.4550002, .3500000,1,1.5707963,0.0,0,0,-2.5000000$,

$4.3249998,0.0,7 \mathrm{HLABEL} 3,0,0$;

$214,2, .1500000, .0500000,0.0,-4.0000000,5.5000000,-2.9000001$,

$4.5000000,-2.5999999,4.5000000,0,0$;

$214,2, .1500000, .0500000,0.0,-4.0000000,1.0000000,-2.9000001$, $4.5000000,-2.5999999,4.5000000,0,0$;

$210,15,2,17,19,0,0$;

$212,1,7,2.4900002, .3500000,1,1.5707963,0.0,0,0,-3.5830004$,

$-1.1750001,0.0,7$ LAEEL $4,0,0$;

$214,2, .1500000, .0500000,0.0,1.5000000,1.0000000,-.6000000$,

$-1.0000000,-.9000000,-1.0000000,0,0$

$214,2, .1500000, .0500000,0.0,1.5000000,-1.0000000,-.6000000$,

$-1.0000000,-.9000000,-1.0000000,0,0$;

$214,2, .1500000, .0500000,0.0,1.5000000,-3.5000000,-.6000000$,

$-1.0000000,-.9000000,-1.0000000,0,0$;

$210,23,3,25,27,29,0,0$;

$212,1,7,2.4550002, .3500000,1,1.5707963,0.0,0,0,11.5000000$, $3.3250000,0.0,7$ HLABEL $5,0,0$;

$214,2, .1500000, .0500000,0.0,5.0000000,5.5000000,7.5000000$,

$3.5000000,11.4000006,3.5000000,0,0 ;$

$210,33,1,35,0,0$;

$212,1,7,2.4550002, .3500000,1,1.5707963,0.0,0,0,5.9169998$,

$-1.6750001,0.0,7$ HLABEL $6,0,0$;

$214 ; 2, .1500000, .0500000,0.0,15.5000000, .5000000,13.0000000$, $-1.5000000,8.6000004,-1.5000000,0,0 ;$

$210,39,1,41,0,0$

5

10

$2 D$

$44 P$

38

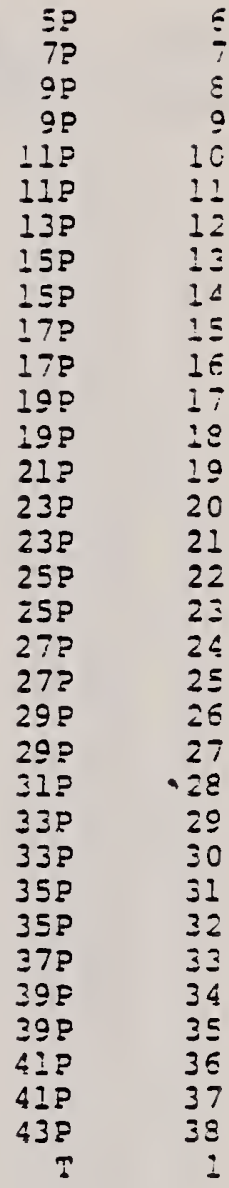


LADEL TEST CASE 2

$1 \mathrm{H}, 1 \mathrm{H} ;, 6 \mathrm{HLABEL2}, 4 \mathrm{HCIIN}, 6 \mathrm{H} 2.1 .3,16,8,24,8,56,1.0000000,1,4 \mathrm{HINCH}, 0,1,13 \mathrm{G}$ 4810827.122851, , , ;

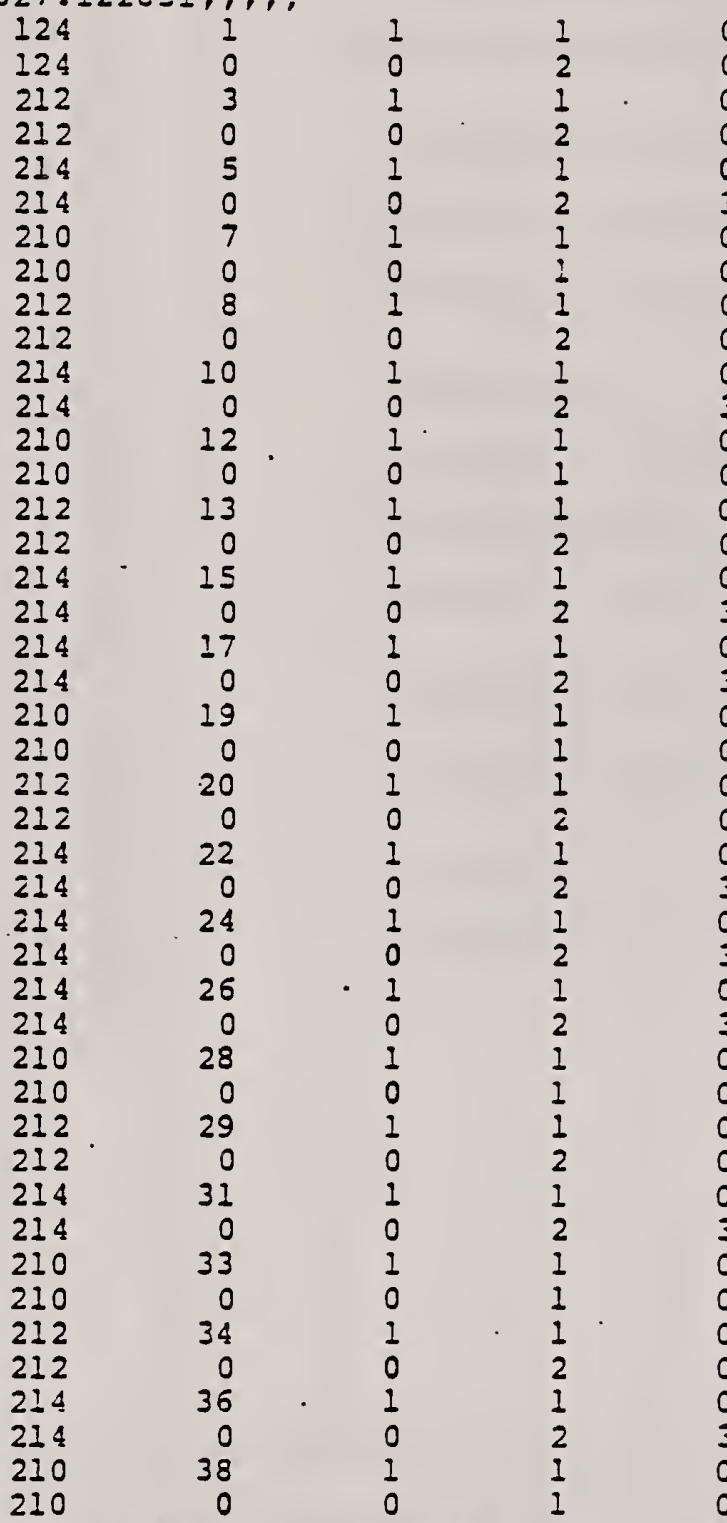

\begin{tabular}{|c|c|}
\hline 0 & 0 \\
\hline 0 & 0 \\
\hline $\begin{array}{l}0 \\
0\end{array}$ & 0 \\
\hline 3 & \\
\hline 0 & 0 \\
\hline 0 & 0 \\
\hline $\begin{array}{l}0 \\
0\end{array}$ & 0 \\
\hline 3 & \\
\hline 0 & 0 \\
\hline $\begin{array}{l}0 \\
0\end{array}$ & 0 \\
\hline 0 & \\
\hline $\begin{array}{l}0 \\
3\end{array}$ & 0 \\
\hline 0 & 0 \\
\hline 3 & \\
\hline $\begin{array}{l}0 \\
0\end{array}$ & 0 \\
\hline 0 & 0 \\
\hline 0 & \\
\hline 3 & 0 \\
\hline $\begin{array}{l}0 . \\
3\end{array}$ & 0 \\
\hline n & 0 \\
\hline $\begin{array}{l}3 \\
0\end{array}$ & 0 \\
\hline 0 & 0 \\
\hline 0 & 0 \\
\hline 0 & 0 \\
\hline 0 & \\
\hline 0 & 0 \\
\hline 0 & 0 \\
\hline $0^{\circ}$ & 0 \\
\hline
\end{tabular}

$\begin{array}{llllllll}0 & 0 & 0 & 0 & 0\end{array}$

$.1564105,-.0027302,-.9876884,0.0, .6859540, .7191879$,

-.1106158,0.0,.7106355,-.6948103,-.1106158,0.0,0,0;

$212,1,7,2.4200002, .3500000,1,1.5707963,0.0,0,0,-10.5480003$, $-1.1750001,0.0,7 \mathrm{HLABEL} 2,0,0$;

、 $214,2, .1500000, .0500000,0.0,-9.0000000,1.0000000,-7.5999999$, 
$-1.0000000,-7.9000001,-1.0000000,0,0$;

$210,3,1,5,0,0$;

$212,1,7,2.2800002, .3500000,1,1.5707963,0.0,0,0,-3.0000000$, $3.8250000,0.0,7$ IAABEL $1,0,0$;

$214,2, .1500000, .0500000,0.0,-9.0000000,5.5000000,-8.3999996$, $4.0000000,-8.1000004,4.0000000,0,0$;

$210,9,1,1,0,0$;

$212,1,7,2.4550002, .3500000,2,1.5707963,0.0,0,0,-2.5000000$, $4.3249998,0.0,7$ ULABEL $3,0,0$;

$214,2, .1500000, .0500000,0.0,-4.0000000,5.5000000,-2.9000001$, $4.5000000,-2.5999999,4.5000000,0,0$;

$214,2, .1500000, .0500000,0.0,-4.0000000,1.0000000,-2.9000001$, $4.5000000,-2.5999999,4.5000000,0,0$; $210,15,2,17,19,0,0$;

$212,1,7,2.4900002, .3500000,1,1.5707963,0.0,0,0,-3.5830004$, $-1.1750001,0.0,7$ HLABEL 4,0,0;

$214,2, .1500000, .0500000,0.0,1.5000000,1.0000000,-.6000000$, $-1.0000000,-.9000000,-1.0000000,0,0$;

$214,2, .1500000, .0500000,0.0,1.5000000,-1.0000000,-.6000000$, $-1.0000000,-.9000000,-1.0000000,0,0$;

$214,2, .1500000, .0500000,0.0,1.5000000,-3.5000000,-.6000000$,

$-1.0000000,-.9000000,-1.0000000,0,0$;

$210,23,3,25,27,29,0,0$;

$212,1,7,2.4550002, .3500000,1,1.5707963,0.0,0,0,11.5000000$, $3.3250000,0.0,7$ HABEL $\equiv, 0,0$;

$214,2, .1500000, .0500000,0.0,5.0000000,5.5000000,7.5000000$, $3.5000000,11.4000006,3.5000600,0,0$

$210,33,1,35,0,0$;

$212,1,7,2.4550002, .3500000,1,1.57,07963,0.0,0,0,5.9169998$, $-1.6750001,0.0,7$ ALABEL $6,0,0$;

$214,2, .1500000, .0500000,0.0,15.5000000, .5000000,13.0000000$, $-1.5000000,8.6000004,-1.5000000,0,0$;

$210,39,1,41,0,0$;

S

IG $2 \mathrm{D}$

$\$ 4 P$

38

\begin{tabular}{rr}
$5 P$ & $E$ \\
$7 P$ & 7 \\
$9 P$ & 8 \\
$9 P$ & 9 \\
$11 P$ & 10 \\
$11 P$ & 11 \\
$13 P$ & 12 \\
$13 P$ & 13 \\
$15 P$ & 14 \\
$17 P$ & 15 \\
$17 P$ & 16 \\
$19 P$ & 17 \\
$19 P$ & 18 \\
$21 P$ & 19 \\
$23 P$ & 20 \\
$23 P$ & 21 \\
$25 P$ & 22 \\
$25 P$ & 23 \\
$27 P$ & 24 \\
$27 P$ & 25 \\
$29 P$ & 26 \\
$29 P$ & 27 \\
$31 P$ & 28 \\
$33 P$ & 29 \\
$33 P$ & 30 \\
$35 P$ & 31 \\
$35 P$ & 32 \\
$37 P$ & 33 \\
$39 P$ & 34 \\
$39 P$ & 35 \\
$41 P$ & 36 \\
$41 P$ & 37 \\
$43 P$ & 38 \\
$T$ & 1 \\
\hline &
\end{tabular}



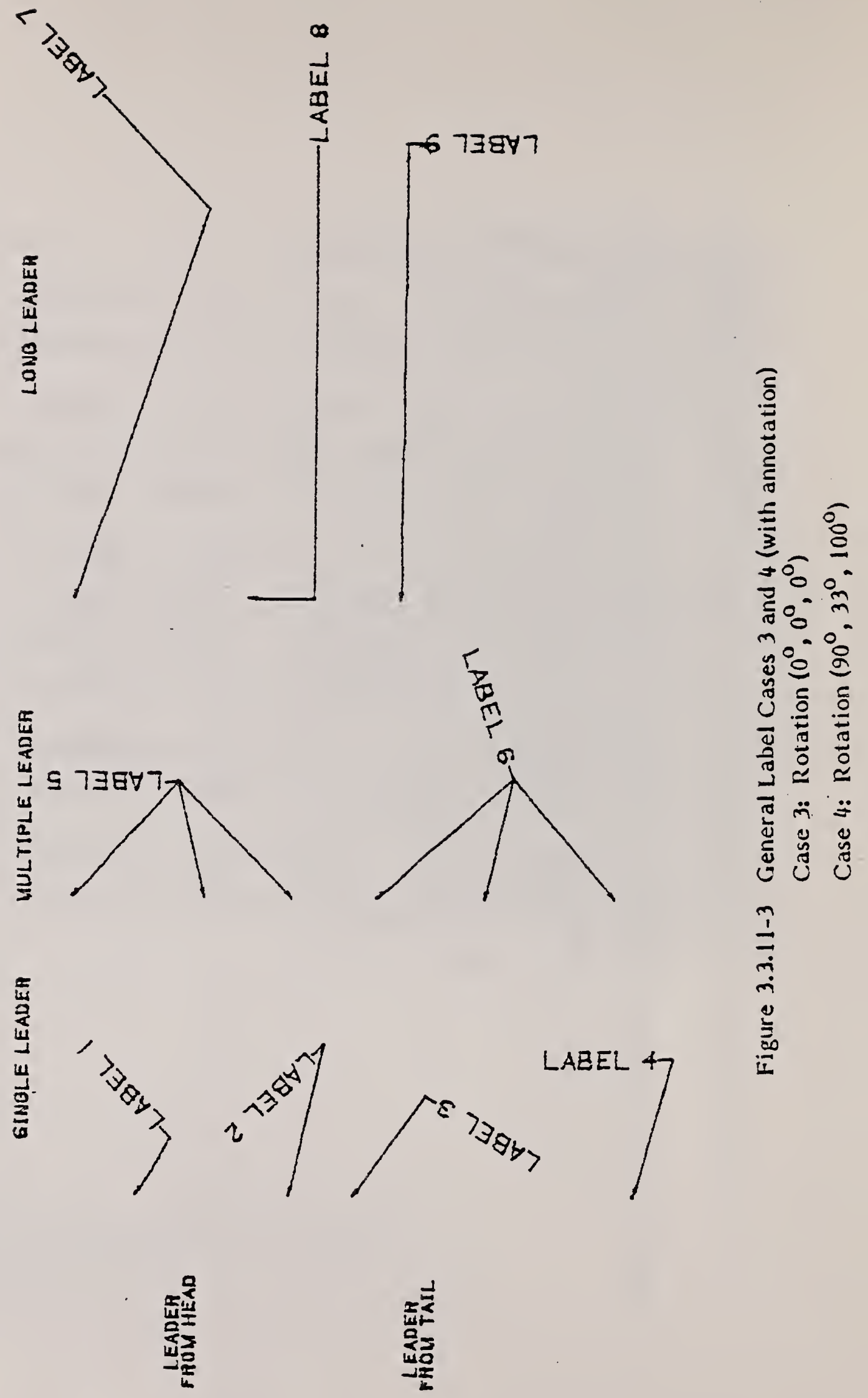

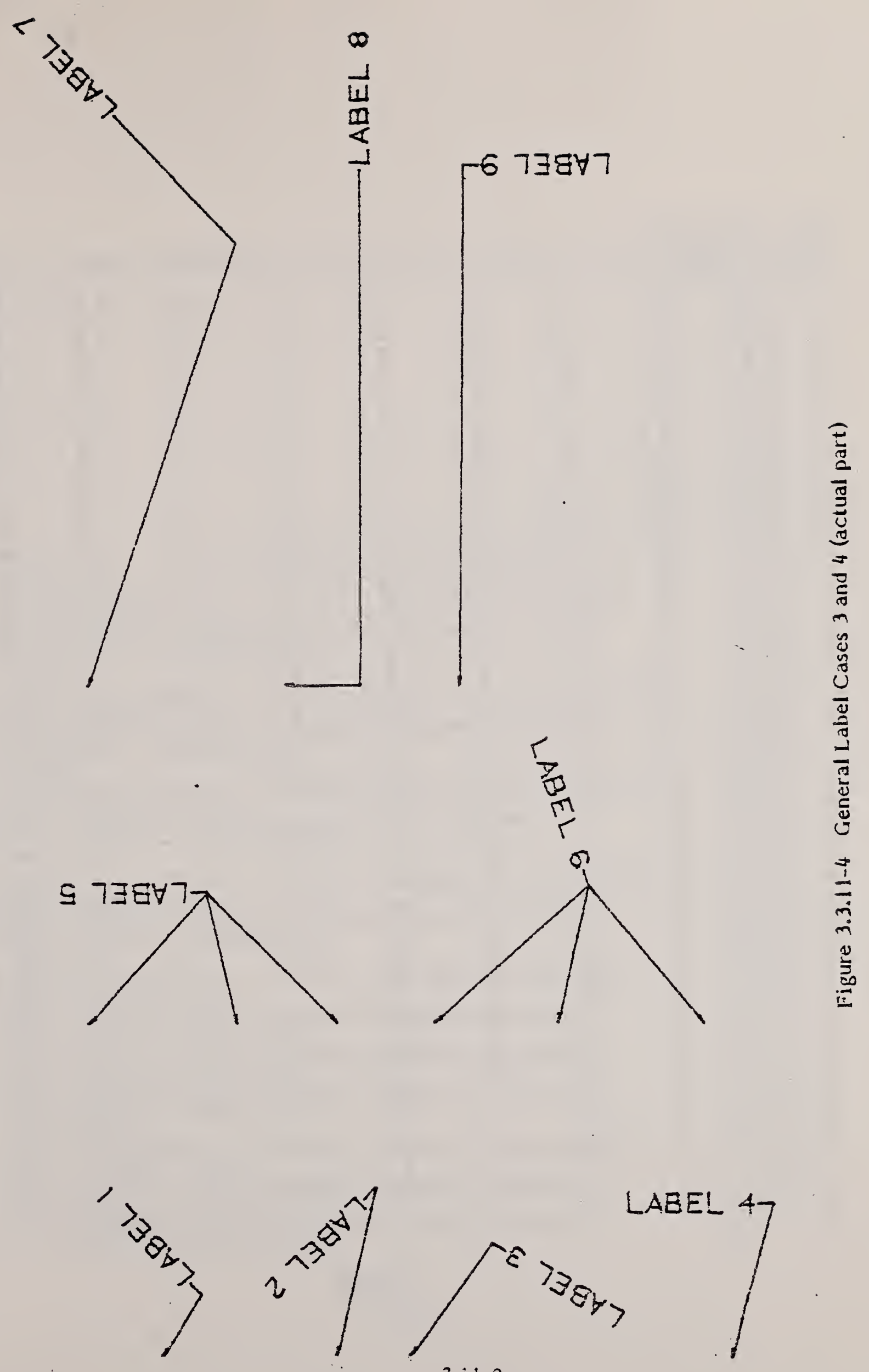

3.11-9 
LABEL TEST CASE 3

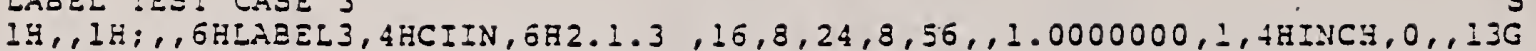

Н810827.122906, ., ;

$\begin{array}{lrrl}124 & 1 & 1 & 1 \\ 124 & 0 & 0 & 2 \\ 212 & 3 & 1 & 1 \\ 212 & 0 & 0 & 2 \\ 214 & 5 & 1 & 1 \\ 214 & -0 & 0 & 2 \\ 210 & 7 & 1 & 1 \\ 210 & 0 & 0 & 1 \\ 212 & 8 & 1 & 1 \\ 212 & 0 & 0 & 2 \\ 214 & 10 ! & 1 & 1 \\ 214 & 0 & 0 & 2 \\ 210 & 12 & 1 & 1 \\ 210 & 0 & 0 & 1 \\ 212 & 13 & 1 & 1 \\ 212 & 0 & 0 & 2 \\ 214 & 15 & 1 & 1 \\ 214 & 0 & 0 & 2 \\ 210 & 17 & 1 & 1 \\ 210 & 0 & 0 & 1 \\ 212 & 18 & 1 & 1 \\ 212 & 0 & 0 & 2 \\ 214 & 20 & 1 & 1 \\ 214 & 0 & 0 & 2 \\ 210 & 22 & 1 & 1 \\ 210 & 0 & 0 & 1 \\ 212 & 23 & 1 & 1 \\ 212 & 0 & 0 & 2 \\ 214 & 25 & 1 & 1 \\ 214 & 0 & 0 & 2 \\ 214 & 27 & 1 & 1 \\ 214 & 0 & 0 & 2 \\ 214 & 29 & 1 & 1 \\ 214 & 0 & 0 & 2 \\ 210 & 31 & 1 & 1 \\ 210 & 0 & 0 & 1 \\ 212 & 32 & 1 & 1 \\ 212 & 0 & 0 & 2 \\ 214 & 34 & 1 & 1 \\ 214 & 0 & 0 & 2 \\ 214 & 36 & 1 & 1 \\ 214 & 0 & 0 & 2 \\ 214 & 38 & 1 & 1 \\ 214 & 0 & 0 & 2 \\ 210 & 40 & 1 & 1 \\ 210 & 0 & 0 & 1 \\ 212 & 41 & 1 & 1 \\ 212 & 0 & 0 & 2 \\ 214 & 43 & 1 & 1 \\ & & & \end{array}$

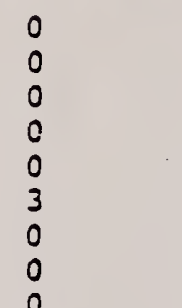

$\begin{array}{lllll}0 & 0 & 0 & 0 & 0 \\ 0\end{array}$

$\begin{array}{llllllll}0 & 1 & 0 & 0 & 1 & 1 & 0\end{array}$

$0.1 \quad 00110$

1

$\begin{array}{lllll}0 & 0 & 0 & 1 & 0\end{array}$

011001100

0

3

(1)

0

3

西

促

$3.11-10$ 


$\begin{array}{lrlll}214 & 0 & 0 & 2 & 3 \\ 210 & 45 & 1 & 1 & 0 \\ 210 & 0 & 0 & 1 & 0 \\ 212 & 46 & 1 & 1 & 0 \\ 212 & 0 & 0 & 2 & 0 \\ 214 & 48 & 1 & 1 & 0 \\ 214 & 0 & 0 & 2 & 3 \\ 210 & 50 & 1 & 1 & 0 \\ 210 & 0 & 0 & 1 & 0 \\ 212 & 51 & 1 & 1 & 0 \\ 212 & 0 & 0 & 2 & 0 \\ 214 & 53 & 1 & 1 & 0 \\ 214 & 0 & 0 & 2 & 3 \\ 210 & 55 & 1 & 1 & 0 \\ 210 & 0 & 0 & 1 & 0 \\ 1000000 & 0.0,0.0,0.0,0.0,1.0000000,0.0,0.0,0\end{array}$

0
0
0
0
0
0
0

$21,1.0000000,0.0,0.0,0.0,0.0,1.0000000,0.0,0.0,0.0,0.0$, $1.0000000,0.0,0,0$;

$212,1,7,2.2800002, .3500000,1,1.5707963, .7853981,0,0,-8.3762560$, $4.3762560,0.0,7$ HLAEEL $1,0,0$;

$214,2, .1500000, .0500000,0.0,-10.0000000,5.0000000,-3.7828425$,

$4.4292893,-8.5707112,4.4292893,0,0$;

$210,3,1,5,0,0 ;$

$212,1,7,2.4200002, .3500000,1,1.5707963,2.3387411,0,0,-6.8741155$, $1.1215653,0.0,7$ :LABEL 2,0,0;

$214,2, .1500000,00500000,0.0,-10.0000000,1.5000000,-6.7221365$,

$.9280560,-6.9305344, .9280660,0,0$;

$210,9,1,11,0,0 ;$

$212,1: 7,2.4550002, .3500000,1,1.5707963,1.0471975,0,0,-9.1390450$,

$-4.3244443,0.0,7$ TASEL $3,0,0$;

$214,2, .2500000, .0500000,0.0,-10.0000000,0.0,-7.7999997$,

$-1.9133974,-7.9499998,-1.9133974,0,0$;

$210,15,1,17,0,0$;

$212,1,7,2.4900002, .3500000,1,1.5707963,4.7123887,0,0,-7.1750002$,

$-4.4169998,0.0,7$ HLABEL $4,0,0$;

$214,2, .1500000, .0500000,0.0,-10.0000000,-6.5000000,-7.0000000$,

$-7.0999999,-7.0000000,-7.0999999,0,0$;

$210,21,1,23,0,0$;

$212,1,7,2.4550002, .3500000,1,1.5707963,1.5707962,0,0,-.8249998$, $4.5000000,0.0,7$ HLABEL $5,0,0$;

$214,2, .1500000, .0500000,0.0,-3.5000000,6.5000000,-1.0000000$,

$4.4000001,-1.0000000,4.4000001,0,0$;

$214,2, .5500000, .0500000,0.0,-3.5000000,3.5000000,-1.0000000$,

$\div$ :

$214,2, .1500000, .0500000,0.0,-3.5000000,1.5000000,-1.0000000$,

$4.400000:-1.0000000,4.4000001,0,0 ;$

$220,27,3,29,31,33,0,0$;

$2 \div 2,1,7,2.4550002, .3500000,1,1.5707963,3.4906584,0,0,1.8673725$,

$-2,4521150,0.0,7$ HLASEL $6,0,0$ :

$2: 4,2, .1500000, .0500000,0.0,-3.5000000,-.5000000,-.8758771$,

$-3.5342021,-.5939693,-3.5342021,0,0$;

$2:-2, . \leq 500000, .0500000,0.0,-3.5000000,-3.0000000,-.8758771$,

$-3.53: 5021,-.5939603,-3.5342021,0,0$;

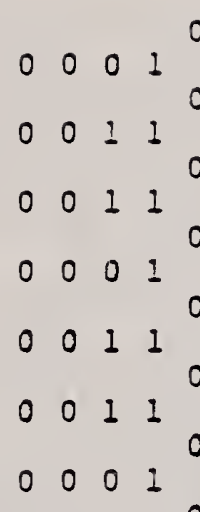

0 D

$C D$

$D$

$D$

OD

$O D$

$D$

OD

$D$

$D$

OD

IP

$3 P$

$3 P$

$E P$

$5 ?$

$7 P$

$9 ?$

9P

IIP

IIP

$13 P$

$15 \%$

$15 \mathrm{P}$

178

i7

IOP

2!?

$21 \mathrm{P}$

$23 \mathrm{P}$

23 ?

$25 P$

278

$27 \mathrm{P}$

$29 \mathrm{P}$

29?

312

$3 I P$

332

33

$35 \mathrm{P}$

$37 P$

37 ?

399

$3 \subseteq ?$

$4 i=$

41 ? 


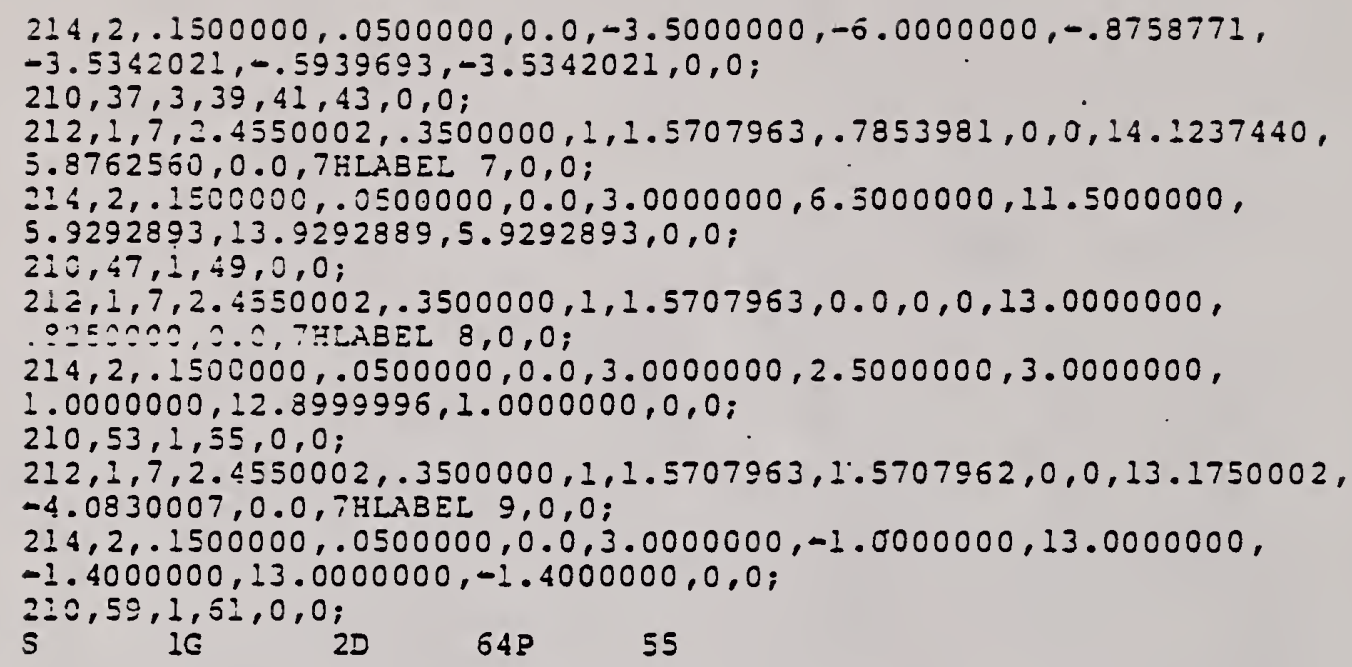


LABEL TEST CASE 4
$1 \mathrm{H}, 1 \mathrm{H} ;, 6 \mathrm{HLABEL} 4,4 \mathrm{HCIIN}, 6 \mathrm{H} 2.1 .3,16,8,24,8,56,1.0000000,1,4 \mathrm{HINCH,0}, 13 \mathrm{~S}$

\$810827.122920, . . . ;

$\begin{array}{lrll}124 & 1 & 1 & 1 \\ 124 & 0 & 0 & 2 \\ 212 & 3 & 1 & 1 \\ 212 & 0 & 0 & 2 \\ 214 & 5 & 1 & 1 \\ 214 & 14 & 0 & 2 \\ 112 & 7 & 1 & 1 \\ 210 & 0 & 0 & 1 \\ 212 & 8 & 1 & 1 \\ 212 & 0 & 0 & 2 \\ 214 & 10 & 1 & 1 \\ 214 & 0 & 0 & 2 \\ 210 & 12 & 1 & 1 \\ 210 & 0 & 0 & 1 \\ 212 & 13 & 1 & 1 \\ 212 & 0 & 0 & 2 \\ 211 & 15 & 1 & 1 \\ 214 & 0 & 0 & 2 \\ 210 & 17 & 1 & 1 \\ 210 & 0 & 0 & 1 \\ 212 & 18 & 1 & 1 \\ 212 & 0 & 0 & 2 \\ 214 & 20 & 1 & 1 \\ 214 & 0 & 0 & 2 \\ 210 & 22 & 1 & 1 \\ 212 & 0 & 0 & 1 \\ 212 & 23 & 1 & 1 \\ 212 & 0 & 0 & 2 \\ 214 & 25 & 1 & 1 \\ 214 & 0 & 0 & 2 \\ 214 & 27 & 1 & 1 \\ 214 & 0 & 0 & 2 \\ 214 & 29 & 1 & 1 \\ 214 & 0 & 0 & 2 \\ 210 & 31 & 1 & 1 \\ 210 & 0 & 0 & 1 \\ 212 & 32 & 1 & 1 \\ 212 & 0 & 0 & 2 \\ 214 & 34 & 1 & 1 \\ 214 & 0 & 0 & 2 \\ 214 & 36 & 1 & 1 \\ 214 & 0 & 0 & 2 \\ 214 & 38 & 1 & 1 \\ 214 & 0 & 0 & 2 \\ 212 & 40 & 1 & 1 \\ 210 & 0 & 0 & 1 \\ 212 & 41 & 1 & 1 \\ 212 & 0 & 0 & 2 \\ 214 & 43 & 1 & 1 \\ & & & \end{array}$

0
0
0
0
0
3
0
0
0
0
0
3
0
0
0
0
0
3
0
0
0
0
0
3
0
0
0
0
0
3
0
3
0
3
0
0
0
0
0
3
0
3
0
3
0
0
0
0
0

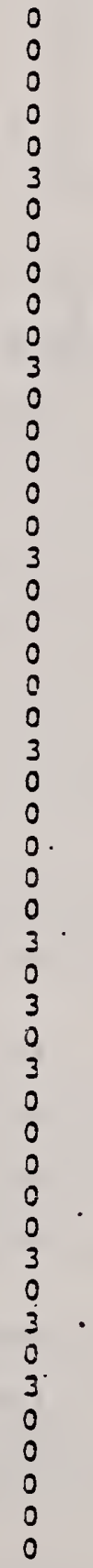

0

2. 1

1

01

i 1

01

0

0

0

1

1

$$
1
$$$$
1
$$

1

$\begin{array}{lllll}0 & 0 & 0 & 0 & 0 \\ 0\end{array}$

$\begin{array}{llllll}0 & 0 & 1 & 1 & 0 & 0\end{array}$

$\begin{array}{llllll}0 & 0 & 1 & 1 & 0 D\end{array}$

$\begin{array}{llllll}0 & 0 & 0 & 1 & 0 & 0\end{array}$

$\begin{array}{llllll}0 & 0 & 1 & 1 & 0 D \\ 0\end{array}$

$\begin{array}{lllll}0 & 0 & 1 & 1 & 0 \\ D\end{array}$

$\begin{array}{llllll}0 & 0 & 1 & 1 & 0 & 0 \\ 0 & 0 & 0 & 0\end{array}$

$\begin{array}{lllll}0 & 0 & 1 & 1 & 0 D\end{array}$

14

OD 16

$\begin{array}{llllll}0 & 0 & 1 & 1 & D & 17\end{array}$

$\begin{array}{lllllll}0 & 0 & 0 & 1 & 0 & 19\end{array}$

$0.110 D \quad 20$

01

01

01

01

0

1

$0,1, O D$

22

23

$001 O D \quad 24$

$\begin{array}{llll}0 & 0 & 0 & 26\end{array}$

$\begin{array}{llllll}0 & 0 & 1 & 1 & D & 27\end{array}$

$\begin{array}{llllll}0 & 0 & 1 & 0 D & D & 28\end{array}$

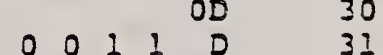

$\begin{array}{llllll}0 & 0 & 1 & 1 & 0 D & 32\end{array}$

$\begin{array}{llllllllll}0 & 1 & 0 & 0 & 1 & 1 & 0 D & 3 \\ 0 & 1 & 0 & 0 & 0 & 1 & 0\end{array}$

$\begin{array}{llllllll}0 & 1 & 0 & 0 & 0 & 1 & D & 35 \\ 0 & 1 & 0 & & & 0\end{array}$

01

$0 \begin{array}{llllll}0 & 0 & 1 & 1 & D & 37\end{array}$

01

o I

01

$\begin{array}{lll}0 & 3 \varepsilon\end{array}$

$\begin{array}{lllllllll}0 & 0 & 1 & 1 & 0 & 39\end{array}$

$001100 \quad 4:$

$00: 100 \quad 4=$

01

$\begin{array}{llll}0 & 0 & 0 & 4\end{array}$

1

0

$i$

$\begin{array}{llllllll}0 & 0 & 1 & 1 & 0 & 0 \\ 0 & 0 & 1 & 0\end{array}$

00120

45

45

49 


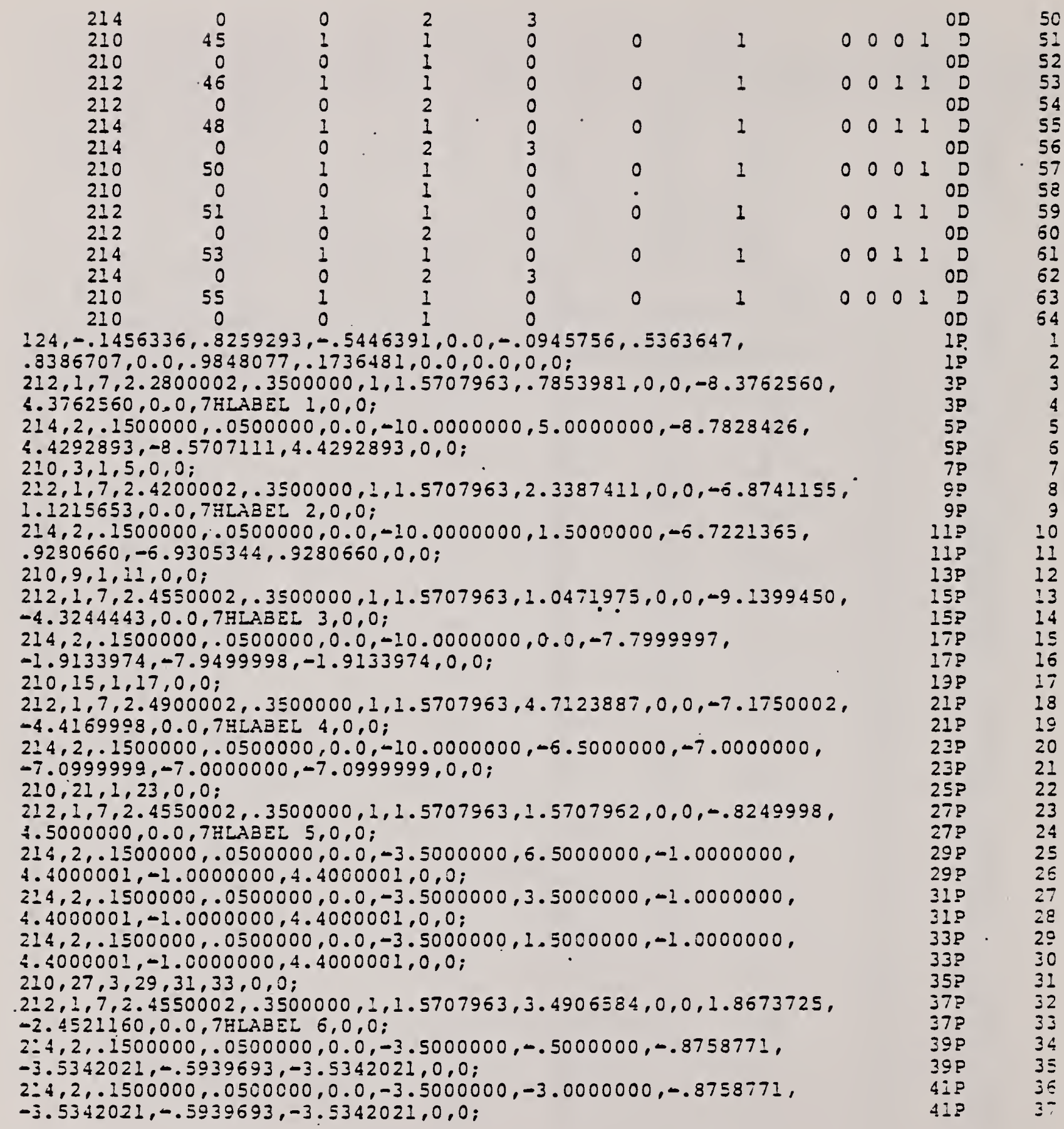


$214,2, .: 500000, .0500000,0.0,-3.5000000,-6.0000000,-.8758771$, $-3 . \equiv 342: 21,-.5939693,-3.5342021,0,0$;

$210,37,3,39,41,43,0,0$;

$212,1,7,2.4550002, .3500000,1,1.5707963, .7853981,0,0,14.1237440$,

$5.8762560,0.0,7:$ LABEL $7,0,0$;

$214,2, .5500000, .0500000,0.0,3.0000000,5.5000000,11.5000000$, $5.9292893,13.9292889,5.9292893,0,0$;

$210,47, \div, 49,0,0$;

$212,1,7,2.4550002, .3500000,1,1.5707963,0.0,0,0,13.0000000$, $.8250000,0.0,7$ THAEEL $8,0,0$;

$214,2, .5 \$ 00000, .0500000,0.0,3.0000000,2.5000000,3.0000000$, $1.0000000,12.8999996,1.0000000,0,0 ;$

$210,53,3,55,0,0$;

$212,1,7,2.4550002, .3500000,1,1.5707963,1.5707962,0,0,13.1750002$, $-4.0830007,0.0,7$ HLABEL $9,0,0$;

$214,2, .1500000, .0500000,0.0,3.0000000,-1.0000000,13.0000000$ ，

$-1.4000000,13.0000000,-1.4000000,0,0$;

$210,59, i, 61,0,0$;

$\mathrm{s}$

16

$2 D$

$64 P$

55

$\begin{array}{rr}43 P & 3 \varepsilon \\ 43 P & 39 \\ 45 P & 4 C \\ 479 & 41 \\ 47 ? & 42 \\ 49 P & 43 \\ 49 P & 44 \\ 51 P & 45 \\ 53 P & 46 \\ 53 P & 47 \\ 55 P & 48 \\ 55 P & 49 \\ 579 & 50 \\ 59 P & 51 \\ 59 P & 52 \\ 61 P & 53 \\ 61 P & 54 \\ 63 P & 55 \\ 7 & 1\end{array}$

\section{$3.11-15$}



The following conventions were followed:

- CV originated text only

- all text has a font characteristic of 1

- slant angle is $0^{\circ}$

- non-mirror condition

Case 1 (Figure 3.3.12-1) tests the following:

- Full alphabetic, numeric, and special character set

- Multi-line text notes

- Text at positive and negative rotation angles

Case 2

- This is icentical to Case 1 except non-model space with rotation $\left(30^{\circ}, 45^{\circ}, 77^{\circ}\right)$ is used per section 3.0

Case 3 (Figures 3.3.12-2 through 3.3.12-5) tests the following:

- large iext heignt (100 in)

- text at positive and negative rotation angles

- long text strings (>400 characters)

- small text height (.001 in)

Case 4

- This is identical to Case 3 except non-model space with rotation $\left(45^{\circ}, 45^{\circ}, 45^{\circ}\right)$ is used per section 3.0 

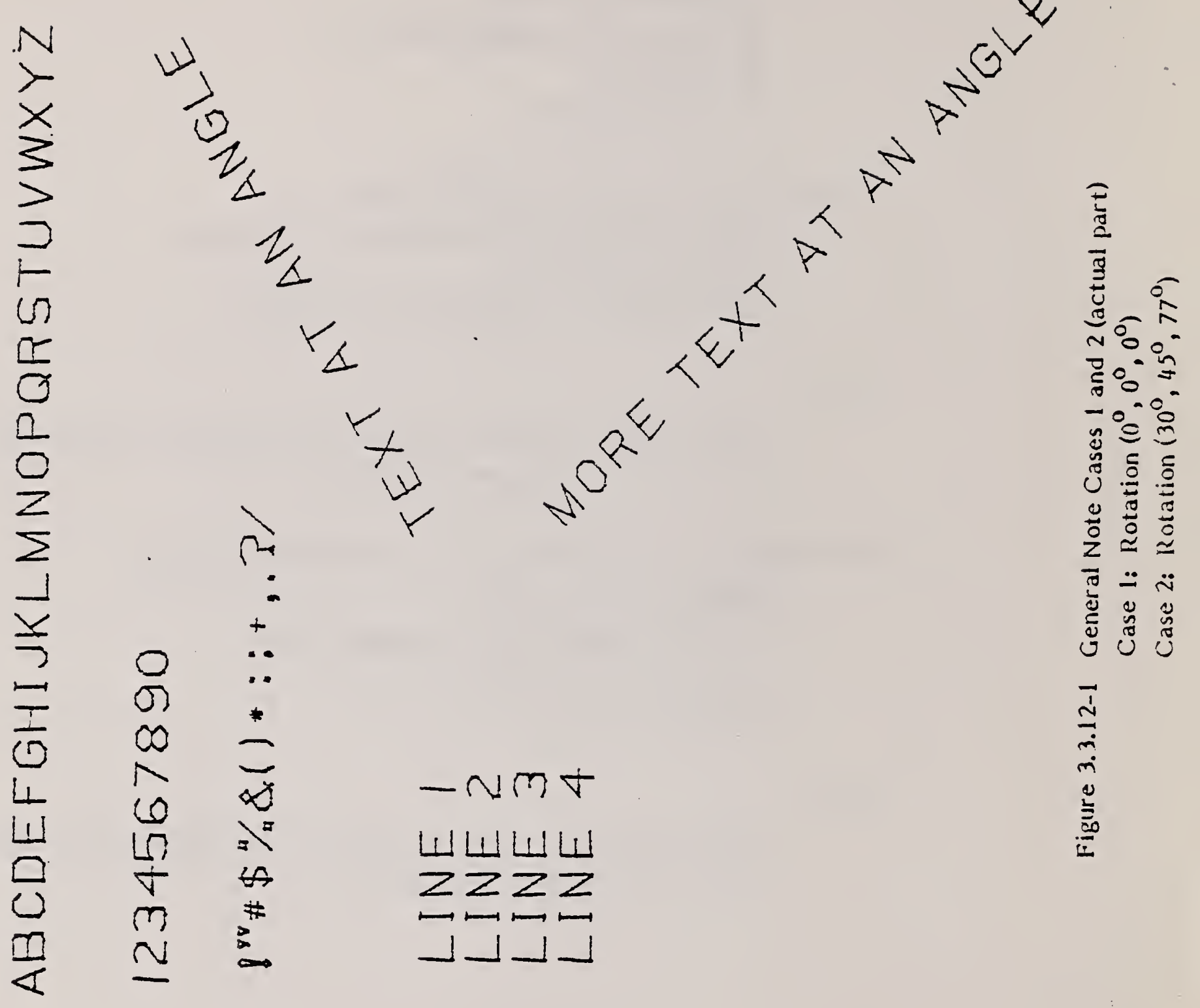

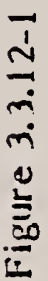


GENERAL NOTE CASE 1

$1 \mathrm{H}, 1 \mathrm{H} ;$, , 6HGNC:E1, $4 \mathrm{HC} I \mathrm{~N}, 6 \mathrm{H} 2.1 .3,16,8,24,3,56,1.0000000,1,4 \mathrm{HINCH}, 0,13 \mathrm{G}$ ה310827.122734, , ,

$\begin{array}{llll}124 & 1 & 1 & 1 \\ 124 & 0 & 0 & 2 \\ 212 & 3 & 1 & 1 \\ 212 & 0 & 0 & 2 \\ 212 & 5 & 1 & 1 \\ 212 & 0 & 0 & 2 \\ 212 & 7 & 1 & 1 \\ 112 & 0 & 0 & 2 \\ 212 & 9 & 1 & 1 \\ 212 & 0 & 0 & 6 \\ 212 & 15 & 1 & 1 \\ 212 & 0 & 0 & 2 \\ 212 & 17 & 1 & 1 \\ 212 & 0 & 0 & 2 \\ 12000000,000.0,0.0,0.0,100\end{array}$

$\begin{array}{ll}1 & 0 \\ 2 & 0 \\ 1 & 0 \\ 2 & 0 \\ 1 & 0 \\ 2 & 0 \\ 1 & 0 \\ 2 & 0 \\ 1 & 0 \\ 6 & 0 \\ 1 & 0 \\ 2 & 0 \\ 1 & 0 \\ 2 & 0\end{array}$

$\begin{array}{lll}0 & 0 & 0 \\ 0 & 0 & 1 \\ 0 & 0 & 1 \\ 0 & 0 & 1 \\ 0 & 1 \\ 0 & 0 & 1 \\ 0 & 0 & 1 \\ 0 & 0 & 1 \\ 0 & 0 & 1 \\ 0 & 0 & 0\end{array}$

$G$ 212

$124,1.0000000,0.0,0.0,0.0,0.0,1.0000000,0.0,0.0,0.0,0.0$, $1,0000000,0.0,0,0 ;$

$2: 2, \therefore, 26,29.4000000,1.0000000,1,1.5707953,0.0,0,0,-9.8846159$, $2.0256412,0.0,25$ AABCDEFGHIJKLANOPQRSTUVWXY 2, 0, 0;

$212,1,10,10.0000000,1.0000000,1,1.5707953,0.0,0,0,-9.5904703$, $-1.9306393,0.0,101: 234567890,0,0$;

$212,1,16,13.1000000,1.0000000,1,1.5707963,0.0,0,0,-9.2979488$, $-\equiv .2945324,0.0,16 \mathrm{~B} ! " \$ \$ 8 \&() *: ;+, . ? /, 0,0$;

$212,4,6,5.2000000,1.0000000,1,1.5707963,0.0,0,0,-3.8445415$,

$-11.1158066,0.0,6 \mathrm{ELINE} 1,6,5.6000000,1.0000000,1,1.5707963,0.0$,

$0,0,-3.844 .541 \equiv,-12.6153066,0.0,6 \operatorname{ALINE} 2,6,5.7000000,1.0000000,1$,

$1.5707963,0.0,0,0,-8.8445415,-14.1158065,0.0,6$ HLINE 3,6 ,

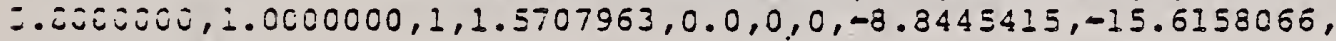

$0.0,6$ HLINE $4,0,0$;

$212,1,16,16.8000000,1.0000000,1,1.5707963, .5235988,0,0$,

$4 . E \sigma 99367,-10.5477728,0.0,16 \mathrm{HTEXT}$ AT AN ANGLE,0,0;

$212,1,21,22.9000000,1.0000000,1,1.5707963,-.7853981,0,0$,

$4.435919 .8,-14.5260605,0.0,21$ HMORE TEXT AT AN ANGLE, 0,0 ;

$\mathrm{S}$

16

$2 D$

$14 P \quad 18$ 
GENERAL NOTE CASE 2

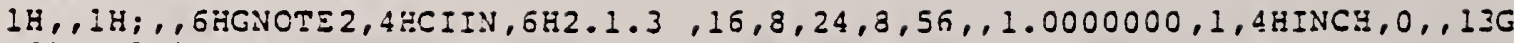

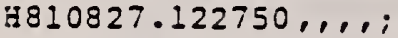

$\begin{array}{rrrr}124 & 1 & 1 & 1 \\ 124 & 0 & 0 & 2 \\ 212 & 3 & 1 & 1 \\ 212 & 0 & 0 & 2 \\ 212 & 5 & 1 & 1 \\ 212 & 0 & 0 & 2 \\ 212 & 7 & 1 & 1 \\ 212 & 0 & 0 & 2 \\ 212 & 9 & 1 & 1 \\ 212 & 0 & 0 & 6 \\ 212 & 15 & 1 & 1 \\ 212 & 0 & 0 & 2 \\ 212 & 17 & 1 & 1 \\ 212 & 0 & 0 & 2\end{array}$

$\begin{array}{ll}1 & 0 \\ 2 & 0 \\ 1 & 0 \\ 2 & 0 \\ 1 & 0 \\ 2 & 0 \\ 1 & 0 \\ 2 & 0 \\ 1 & 0 \\ 6 & 0 \\ 1 & 0 \\ 2 & 0 \\ 1 & 0 \\ 2 & 0\end{array}$

$\begin{array}{lll}0 & 0 & 0 \\ 0 & 0 & 1 \\ 0 & 0 & 1 \\ 0 & 0 & 1 \\ 0 & 0 & 1 \\ 0 & 0 & 1 \\ 0 & 0 & 1 \\ 0 & 0 & \end{array}$

$\begin{array}{lllll}0 & 0 & 0 & 0 & 0\end{array}$

$G$
$D$

$124, .1590645, .6889837,-.7071068,0.0,-.7642970, .5393053, .3535534$, $0.0, .6249390, .4842018, .5123725,0.0,0,0$;

$212,1,26,29.4000000,1.0000000,1,1.5707963,0.0,0,0,-9.8846159$, $2.0256412,0.0,26$ HABCDEEGHIJKLYNCPQRSTUVWKY Z, 0,0 ;

$212,1,10,10.0000000,1.0000000,1,1.5707963,0.0,0,0,-9.5904703$, $-1.9306393,0.0,10 \mathrm{H} 1234567890,0,0$;

$212,1,16,13.1000000,1.0000000,1,1.5707963,0.0,0,0,-9.2979488$, $-5.2945324,0.0,16 \mathrm{H} ! "+\$ 3 \&() \div: ;+, . ? /, 0,0 ;$

$212,4,6,5.2000000,1.0000000,1,1.5707963,0.0,0,0,-2.8445415$,

$-11.1153066,0.0,6$ RLIVE $1,6,5.6000000,1.0000000,1,1.5707963,0.0$, $0,0,-8.8445415,-12.61 \equiv 8066,0.0,6$ IIINE $2,6,5.7000000,1.0000000,1$, $1.5707953,0.0,0,0,-3.3445415,-14.1158066,0.0,6$ GIINE 3,6,

$5.8000000,1.0000000,1,1.5707963,0.0,0,0,-8.8445415,-15.6158066$, $0.0,6$ ZLINE 4,0,0;

$212,1,16,16.8000000,1.0000000,1,1.5707953, .5235988,0,0$, $4.6699367,-10.6477723,0.0,16 \mathrm{HTEXT}$ AT AN ANGLE, 0,0;

$212,1,21,22.4000000,1.0000000,1,1.5707963,-.7853981,0,0$, $4.4359198,-14.6260605,0.0,21$ HMORE TEXT AT AN ANGLE, 0,0 ;

$\mathrm{S}$ IG $2 \mathrm{D}$ I $2 \mathrm{P}$ 18 


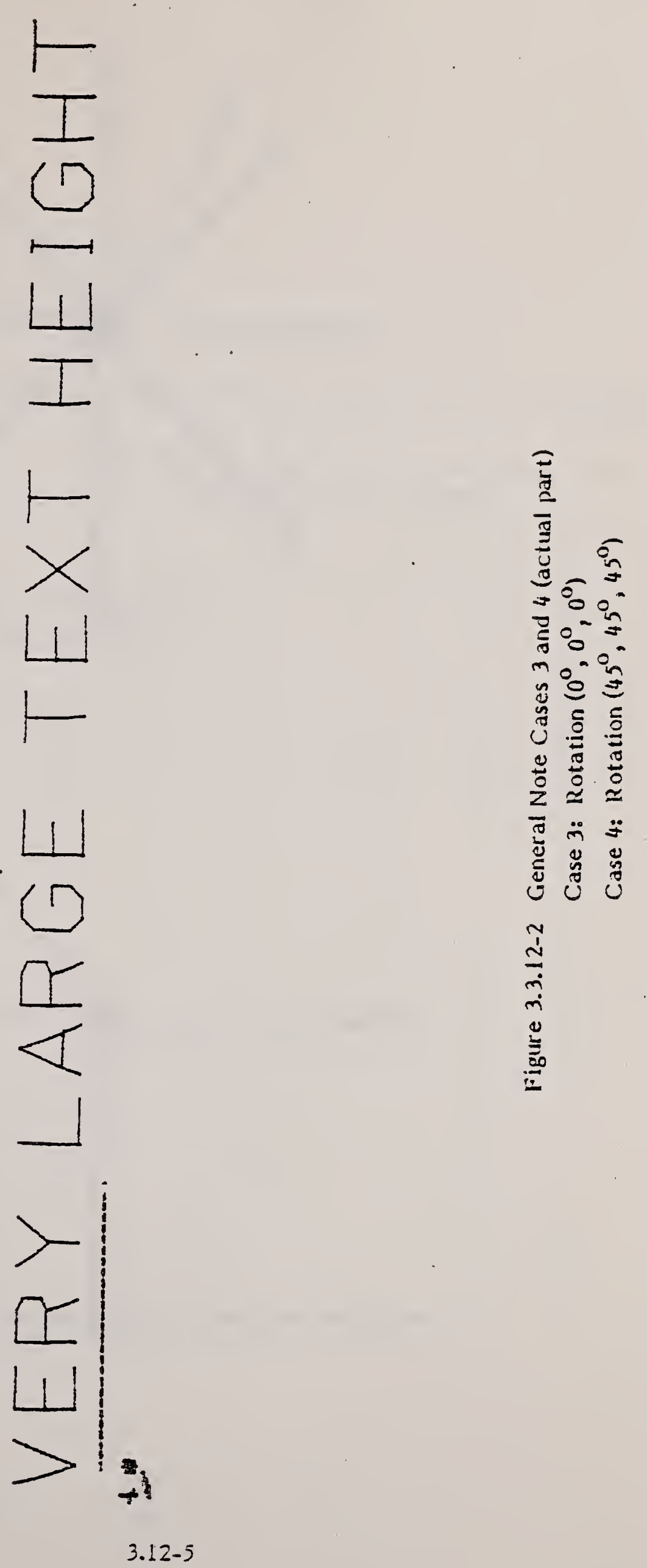




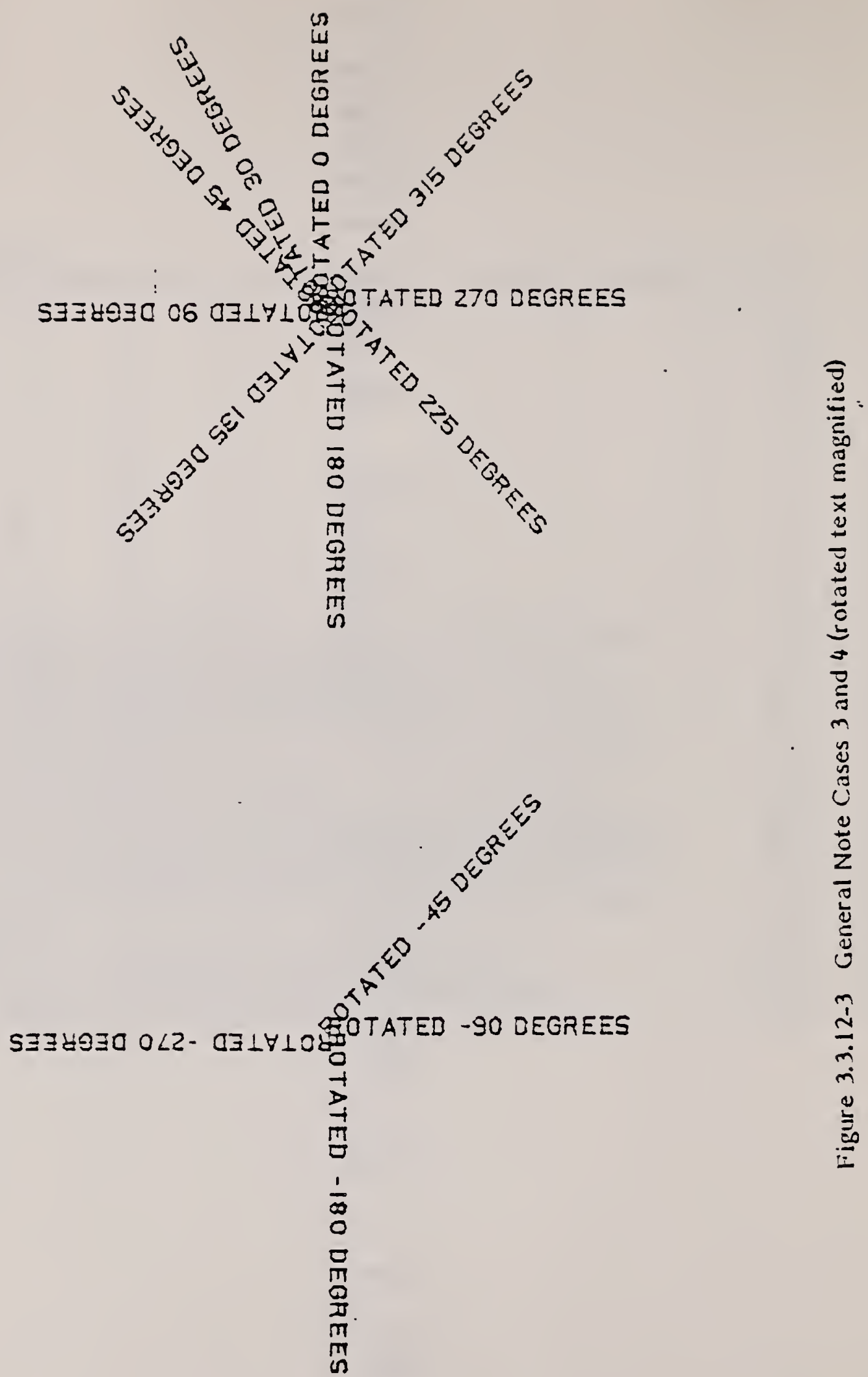


THIS IS A VERY LONG CHARACTER STRINGGGG
Note: Not aii the "G's" in this note
are shown in this figure

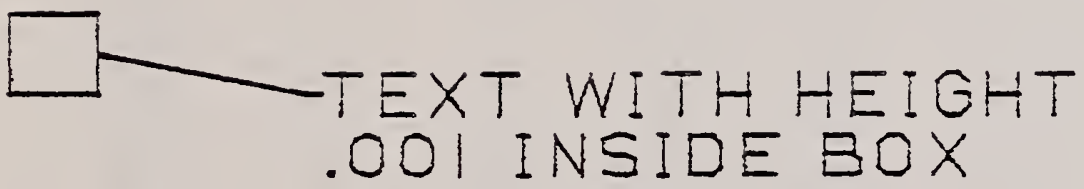

Figure 3.3.12-4 General Note Cases 3 and 4 (long text magnified) 
VERY SMALL TEXT HEIGHT

Figure 3.3.12-5 General Note Cases 3 and 4 (small text magnified) 
GENERAL NOTE CASE 3
1H, $1 \mathrm{H} ;, 6 \mathrm{HGNOTE} 3,4 \mathrm{HCINN}, 6 \mathrm{H} 2.1 .3,16,8,24,8,56,1,0000000,1,4 \mathrm{HINC}, 0,1,13 \mathrm{~S}$ :810827.122803, , ,

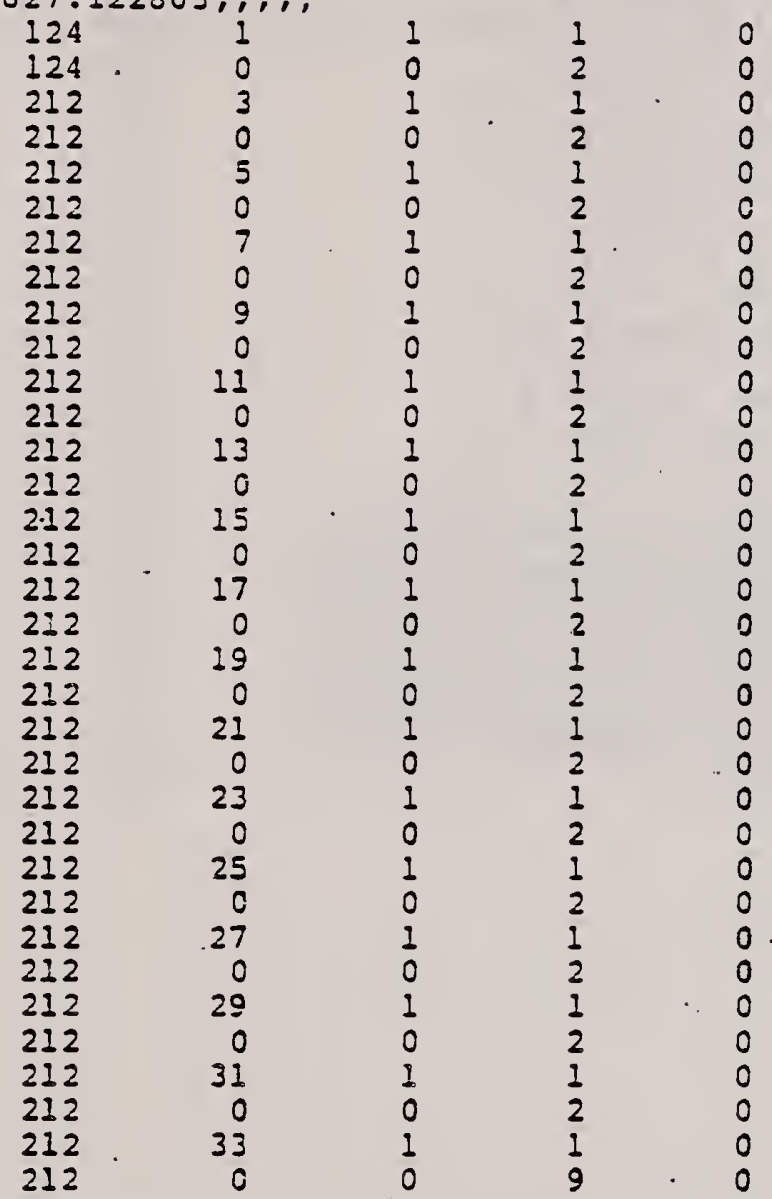

$\begin{array}{lllllllll}0 & 0 & 0 & 0 & D\end{array}$

$\begin{array}{llllll}0 & 0 & 0 & 1 & D\end{array}$

$\begin{array}{llllllll}0 & 1 & 0 & 0 & 0 & 1 & D \\ 0 & 0 & 0 & 0 & 0 & 0 & 0\end{array}$

0

$\begin{array}{llllll}0 & 0 & 0 & 1 & 0\end{array}$

01

$\begin{array}{lllllllll}0 & 0 & 0 & 1 & 0\end{array}$

01

$\begin{array}{lllll}0 & 0 & 0 & 1 & 0\end{array}$

- 1

$0 \begin{array}{llll}0 & 0 & 0 & 0\end{array}$

01

OD

$\begin{array}{lllll}0 & 0 & 0 & 1 & 0\end{array}$

01

$\begin{array}{lllllllll}0 & 0 & 0 & 1 & D\end{array}$

o 2

$\begin{array}{lllll}0 & 0 & 0 & 1 & 0\end{array}$

$\begin{array}{lllll}0 & 0 & 0 & 1\end{array}$

$\begin{array}{ll}0 & 1 \\ 0 & 1\end{array}$

000, OD

$\begin{array}{lll}0 & 0 & 0\end{array}$

1

$\begin{array}{ll}0 & 1 \\ 0 & 1\end{array}$

OD

$\begin{array}{lllll}0 & 0 & 0 & 1 & D\end{array}$

0 O 00110

$\begin{array}{lllll}0 & 0 & 0 & 1 & D\end{array}$

1

01

0

$\begin{array}{lllll}0 & 0 & 0 & 1 & 0\end{array}$

0001 OD

$\begin{array}{lll}0 & 3 \\ 0 & 33\end{array}$

$124,1.0000000,0.0,0.0,0.0,0.0,1.0000000,0.0,0.0,0.0,0.0$, $1.0000000,0.0,0,0$;

$212,1,22,2340.0000000,100.0000000,1,1.5707963,0.0,0,0$, -10.7564106,2.0255412,0.0,225VERY LARGE TEXT HEIGHT,0,0; $212,1,22, .0236000, .0010000,1,1.5707963,0.0,0,0,14.1943779$, $-50.5557480,0.0,22$ GVERY SMALE TEXT HEIGHT,0,0;

$212,1,17,18.4000000,1.0000000,1,1.5707963,0.0,0,0,19.0201511$, -0.9595032,0.0,17HROTATED O DEGREES,0,0;

$212,1,18,19.5000000,1.0000000,1,1.5707963, .5235988,0,0$, $19.0201511,-90.9595032,0.0,18$ ERCTATED 30 DEGREES, 0,0 ; $212,1,18,19.5000000,1.0000000,1,1.5707963, .7853981,0,0$, $19.02015: 1,-90.9595032,0.0,134 R O T A T E D 45$ DEGREES, 0,0 ; $212,1,18,19.5000000,1.0000000,1,1.5707963,1.5707963,0,0$, $19.0201511,-90.9595032,0.0,18$ UROTATED 90 DEGRESS,0,0; $212,1,19,20.2000000,1.0000000,1,1.5707 .963,2.3561345,0,0$, 
$19.0201511,-90.9595032,0.0,19$ HROTATED 135 DEGREES, 0,0 ; $212,1,19,20.3000000,1.0000000,1,1.5707963,3.1415925,0,0$, $19.0201511,-90.9595032,0.0,19$ HROTATED 180 DESREES,0,0; $212,1,19,20.4000000,1.0000000,1,1.5707963,3.9269907,0,0$, $19.0201511,-90.9595032,0.0,19$ HROT:TED 225 DEGREES, 0,0; $212,1,19,20.5000000,1.0000000,1, \dot{2} 5707953,4.7123890,0,0$, $19.0201511,-90.9595032,0.0,19$ HROTATED 270 DEGREES,0.0; $212,1,19,20.2000000,1.0000000,1,1.5707953,5.4977870,0,0$, $19.0201511,-90.9595032,0.0,19$ ERCTATED 315 DEEREES,0,0; . $212,1,19,20.6000000,1.0000000,1,1.5707063,-.7853981,0,0$, $-27.3520813,-91.0622711,0.0,19$ HROTATED -45 DEGREES,0,0; $212,1,19,20.6000000,1.0000000,1,1.5707963,-1.5707963,0,0$, $-27.3520813,-91.0622711,0.0,19$ HRCTATED -90 DEGREES, 0.0 ; $212,1,20,21.4000000,1.0000000,1,1.5707963,-3.1415925,0,0$, $-27.3520813,-91.0622711,0.0,20$ GROTATED -180 DEGREES, 0,0; $212,1,20,21.6000000,1.0000000,1,1.5707963,-4.7123890,0,0$, $-27.3520813,-91.0622711,0.0,20$ HROTATED -270 DEGREES,0,0; $212,1,470,514.9000000,1.0000000,1,1.5707963,0.0,0,0,13.5163784$, $-44.0563965,0.0,470 H T H$ IS IS A VERY LONG CHARACTER STRINGGGGGSGGS

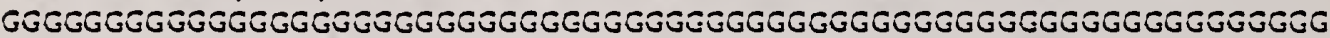

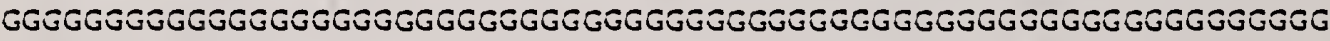

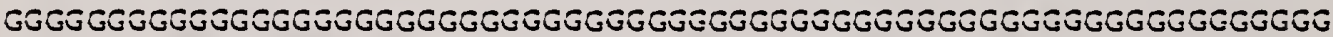

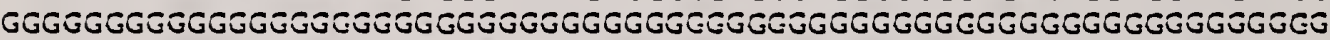

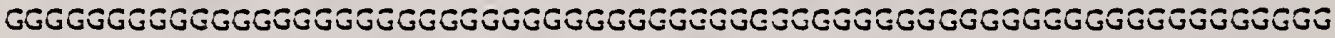

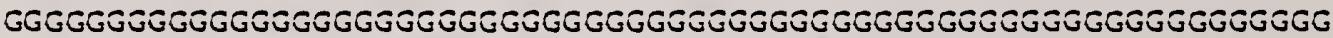
GSGGGGSUSGGGSGSESGESGGGGGGGGGGGSGUGGSEUGSU, 0,0 ; $\mathbf{s}$ 16 2D $34 \mathrm{P}$ 41

\begin{tabular}{rr}
$15 P$ & $1 E$ \\
$17 P$ & 17 \\
$17 P$ & 18 \\
$19 P$ & 19 \\
$19 P$ & 20 \\
$21 P$ & 21 \\
$21 P$ & 22 \\
$23 P$ & 23 \\
$23 P$ & 24 \\
$25 P$ & 25 \\
$25 P$ & 25 \\
$27 P$ & 27 \\
$27 P$ & 28 \\
$29 P$ & 29 \\
$29 P$ & 30 \\
$31 P$ & 31 \\
$31 P$ & 32 \\
$33 P$ & 33 \\
$33 P$ & 31 \\
$33 P$ & 35 \\
$33 P$ & 35 \\
$33 P$ & 37 \\
$33 P$ & 38 \\
$33 P$ & 39 \\
$33 P$ & 40 \\
$33 P$ & 41 \\
$T$ & 1 \\
\hline
\end{tabular}


GENERAL NOTE CASE 4 G H810827.122824, , , ;

$\begin{array}{lll}124 & 1 & 1 \\ 124 & 0 & 0 \\ 212 & 3 & 1 \\ 212 & 0 & 0 \\ 212 & 5 & 1 \\ 212 & 0 & 0 \\ 212 & 7 & 1 \\ 212 & 0 & 0 \\ 212 & 9 & \vdots \\ 212 & 0 & 0 \\ 212 & 11 & 1 \\ 212 & 0 & 0 \\ 212 & 13 & 1 \\ 212 & 0 & 0 \\ 212 & 15 & 1 \\ 212 & 0 & 0 \\ 212 & 17 & 1 \\ 212 & 0 & 0 \\ 212 & 19 & 1 \\ 212 & 0 & 0 \\ 212 & 21 . & 1 \\ 212 & 0 & 0 \\ 212 & 23 & 1 \\ 212 & 0 & 0 \\ 212 & 25 & 1 \\ 212 & 0 & 0 \\ 212 & 27 & 1 \\ 212 & 0 & 0 \\ 212 & 29 & 1 \\ 212 & 0 & 0 \\ 212 & 31 & 1 \\ 212 & 0 & 0 \\ 212 & 33 & 1 \\ 212 & 0 & 0 \\ 50000012 & 500000 & 0 \\ 12 & 07\end{array}$

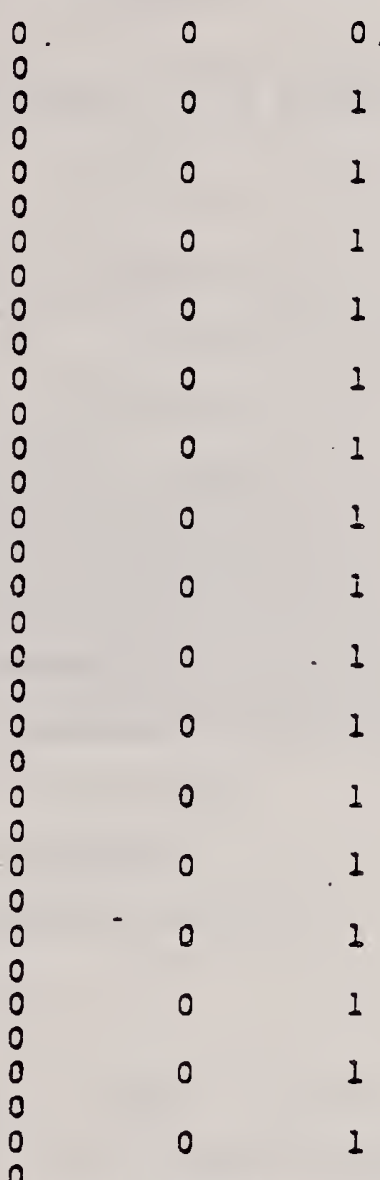

$124, .5000001, .5000000,-.7071068,0.0,-.1464466, .8535534, .5000000$, $0.0, .8535534,-.1464466, .5000001,0.0,0,0$;

$212,1,22,2340.0000000,100.0000000,1,1.5707963,0.0,0,0$, $-10.7564205,2.0256412,0.0,22 \mathrm{EVERY} \mathrm{LARGE} \mathrm{TEXT} \mathrm{HEIGHT,0,0;}$ $212,1,22, .0236000, .0010000,1,1.5707963,0.0,0,0,14.1943779$, $-50.5557480,0.0,22$ HVERY SMALL TEXT HEIGHT,0,0;

$212,1,17,18.4000000,1.0000000,1,1.5707963,0.0,0,0,19.0201511$, $-90.9595032,0.0,17$ IROTATEJ O DEGREES, 0,$0 ;$.

$212,1,13,19.5000000,1.0000000,1,1.5707963, .5235988,0,0$, $19.0201511,-90.9595032,0.0,18$ HROTATED 30 DEGREES, 0,0 ; $212,1,18,19.5000000,1.0000000,1,1.5707963, .7853931,0,0$, $19.0201511,-90.9595032,0.0,18$ HROTATED 45 DEGREES, 0,0 ;

$212,1,18,19.5000000,1.0000000,1,1,5707953,1.5707963,0,0$, $19.0201512,-90.9595032,0.0,18$ HROTATED 90 DEGREES, 0,0 ; $212,1,19,20.2000000,1.0000000,1,1.5707963,2.3561945,0,0$, 
$19.0201511,-90.9595032,0.0,19$ HRCTATED 135 DECRESS, 0,0 ; $212,1,19,20.3000000,1.0000000,1,1.5707963,3.1415925,0,0$, $19.0201511,-90.9595032,0.0,19$ HROTATED 180 DEGRESS,0,0; $212,1,19,20.4000000,1.5000000,1,1.5707963,3.9259907,0,0$, $19.0201511,-90.9595032,0.0,19$ HROTATED 225 DEGREES, 0,0; $212,1,19,20.5000000,1.0000000,1,1.5707963,4.7123890,0,0$, $19.0201511,-90.9595032,0.0,19$ HROTATED 270 DEGRESS, 0,0; $212,1,19,20.2000000,1.0000000,1,1.5707963,5.4977870,0,0$, $19.0201511,-90.9595032,0.0,19$ HROTATED 315 DEGREES, 0,0; $212,1,19,20.6000000,1.0000000,1,1.5707963,-.7853981,0,0$, $-27.3520813,-91.0622711,0.0,19$ HROTATED -45 DEGRESS, 0,0 ; $212,1,19,20.6000000,1.0000000,1,1.5707963,-1.5707 .963,0,0$, $-27.3520813,-91.0622711,0.0,19$ EROTATED -90 DEGREES,0,0; $212,1,20,21.4000000,1.0000000,1,1.5707963,-3.1415925,0,0$, $-27.3520813,-91.0622711,0.0,20$ HROTATED -180 DEGREES, 0,0 ; $212,1,20,21.6000000,1.0000000,1,1.5707963,-4.7123890,0,0$, $-27.3520813,-91.0622711,0.0,20$ HROTATED -270 DEGREES, 0,0; $212,1,470,514.9000000,1.0000000,1,1.5707963,0.0,0,0,13.5163784$, $-44.0563965,0.0,470$ HTH IS IS A VERY LONG CHARACTER STRINGGGGGGGGG

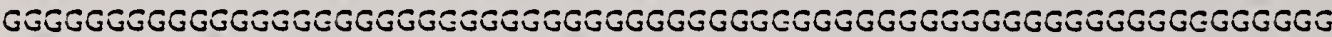

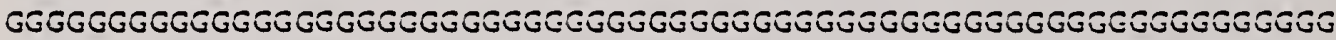

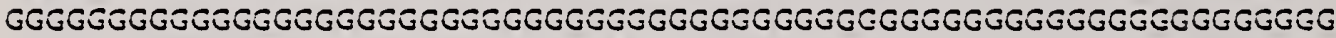

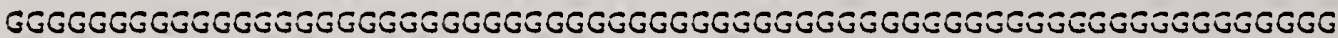

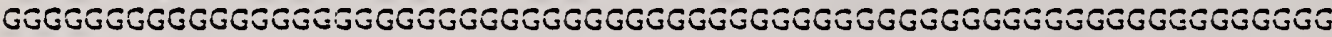

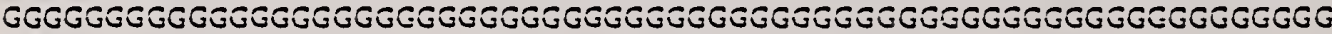

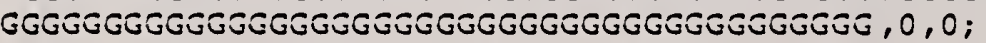




\subsubsection{Linear Dimension}

The linear dimensions contain subentities which include leaders, witness lines and general notes.

The following conventions were followed:

- general note sub-entities to be single text string with no box rotation

Case 1 (Figures $3.3 .13-1$ through $3.3 .13-4$ ) tests the following:

- text in, arrows in configuration

- text in, arrows out configuration

- text out, arrows out configuration

- text out, arrows in configuration

- no witness lines suppressed

- first witness line suppressed

- $\quad$ second witness line suppressed

- both witness lines suppressed

- vertical and horizontal orientations

Case 2

- This is identical to Case 1 except non-model space with rotation $\left(-40.80^{\circ},-7.24^{\circ},-8.31^{\circ}\right)$ is used per Section 3.0 

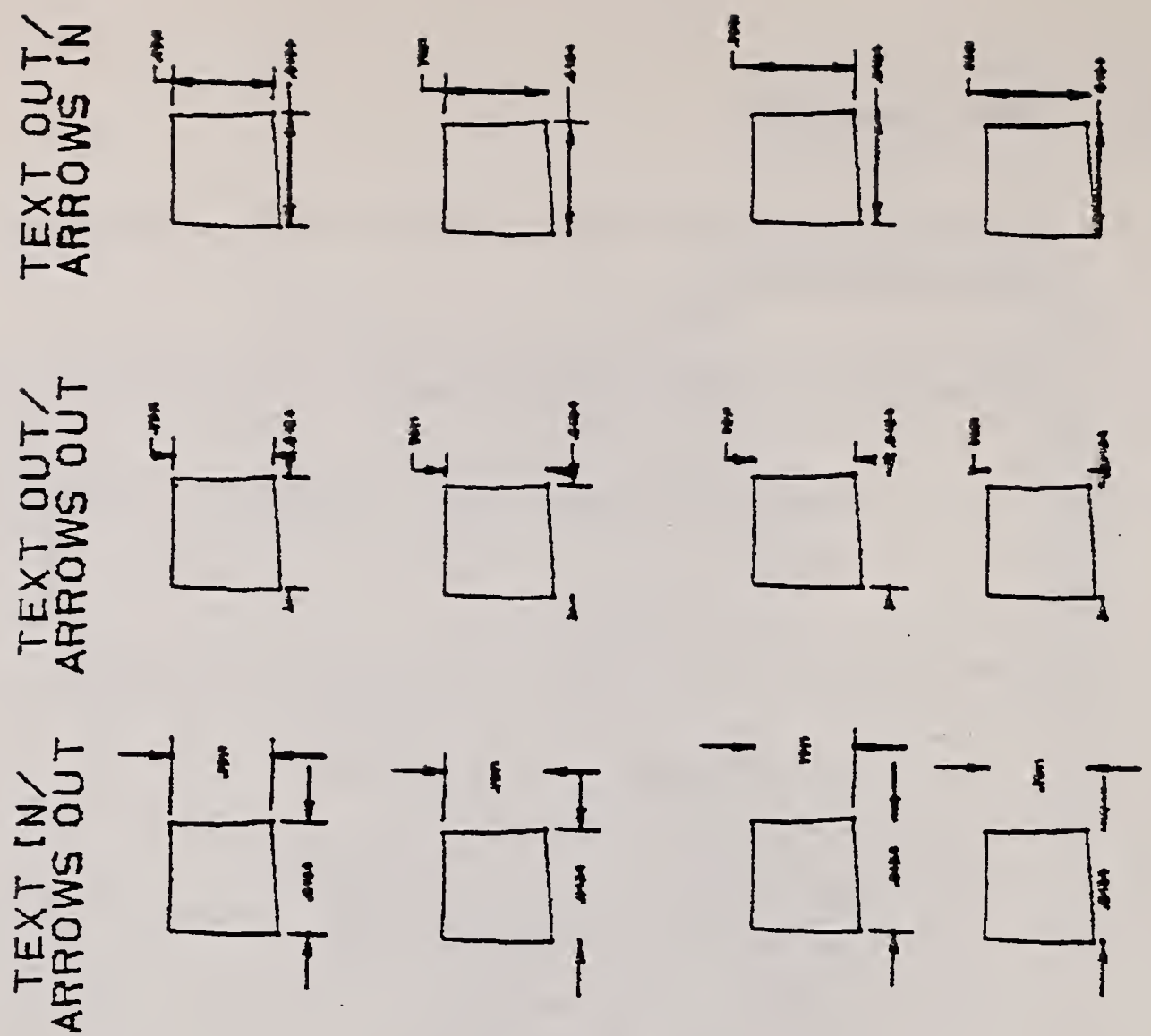

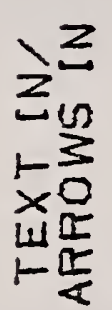
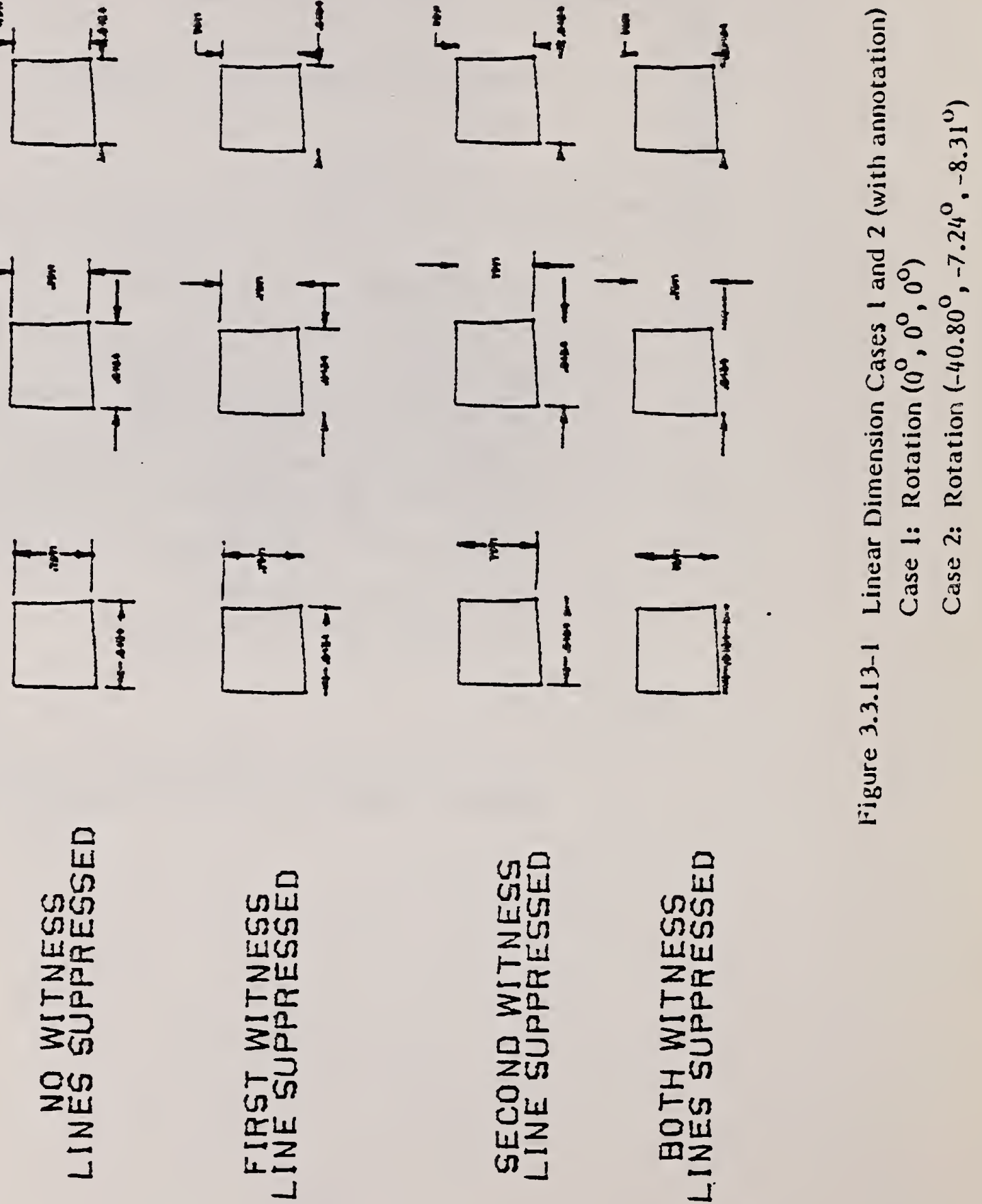

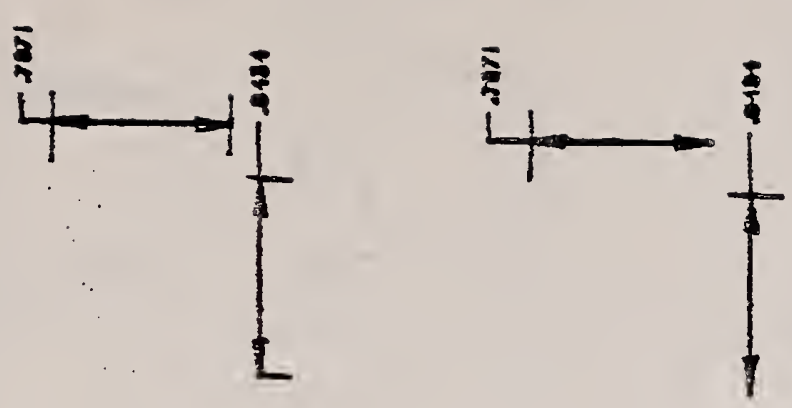

4

5

$\rightarrow \frac{1}{1}$

$\pm$

1

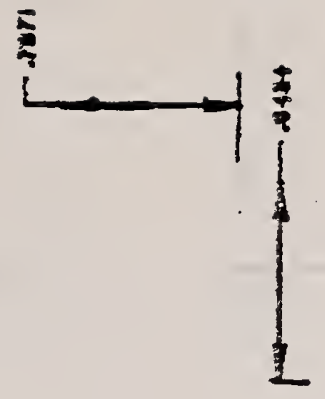

L L L

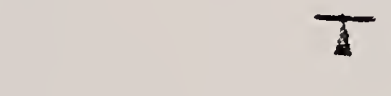

$\rightarrow$

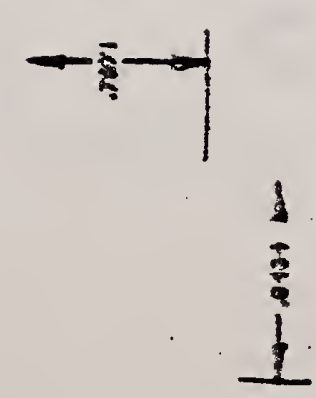

$T$

$T$

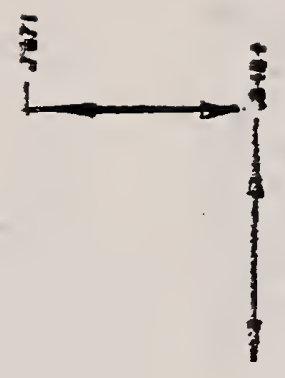

3

$\frac{1}{2} \frac{7}{3}$

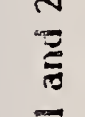

10

$\frac{5}{n}$

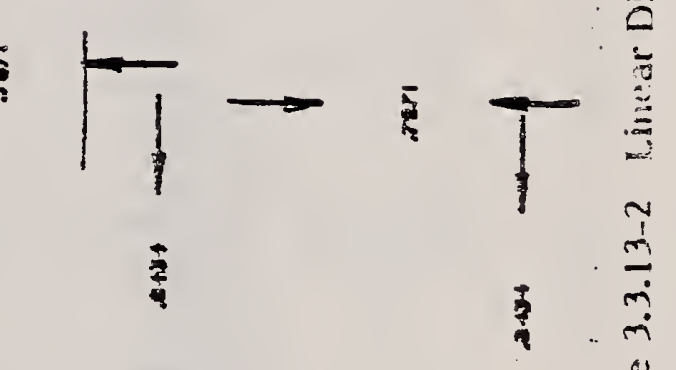

1 总
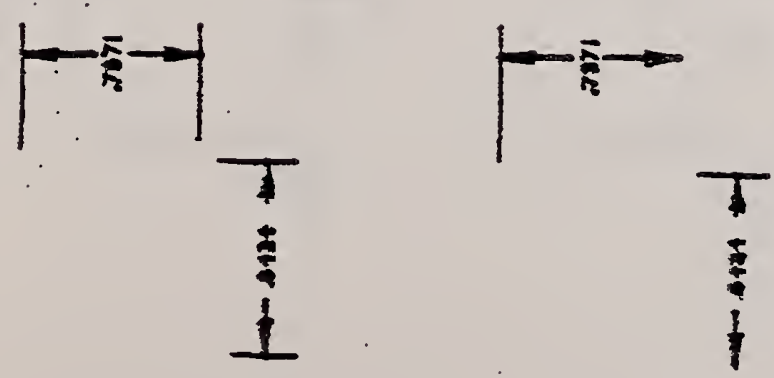

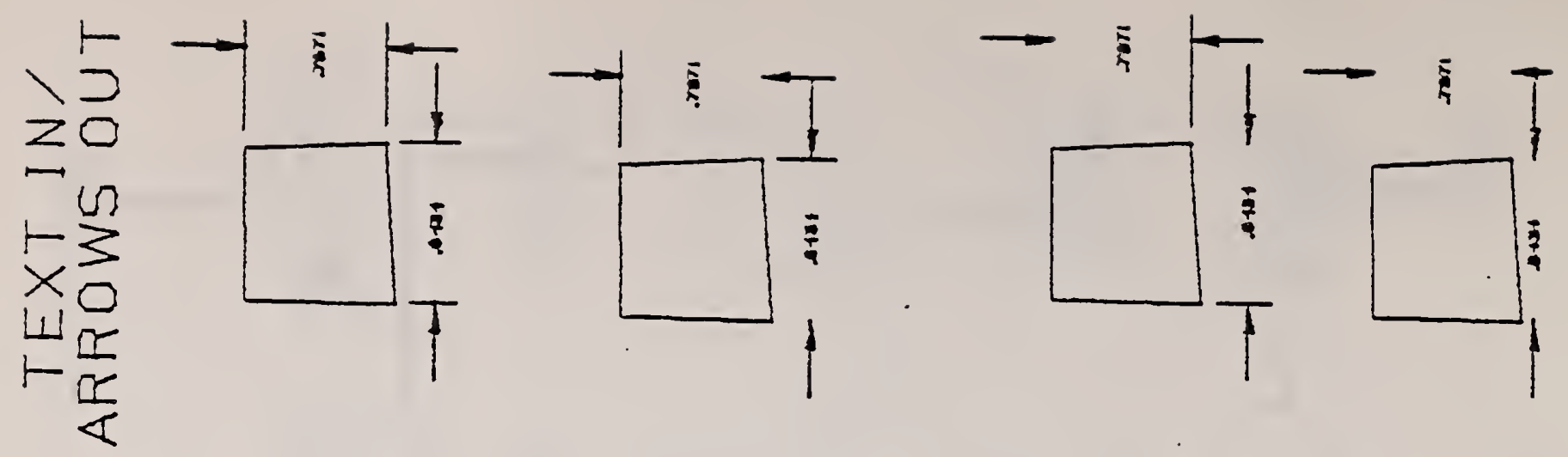

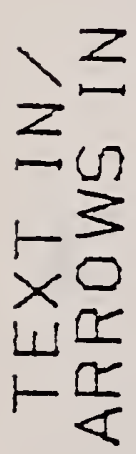
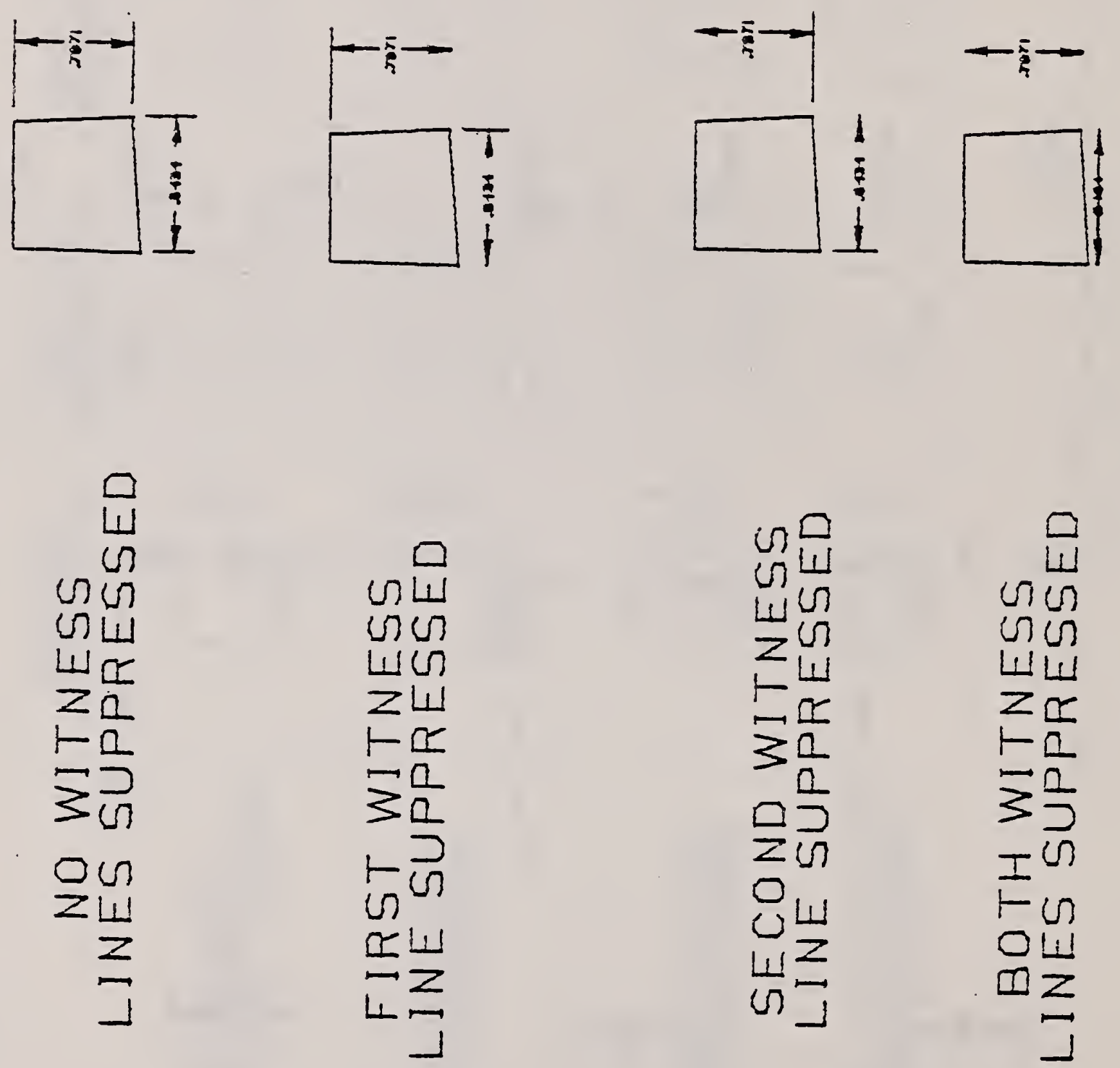

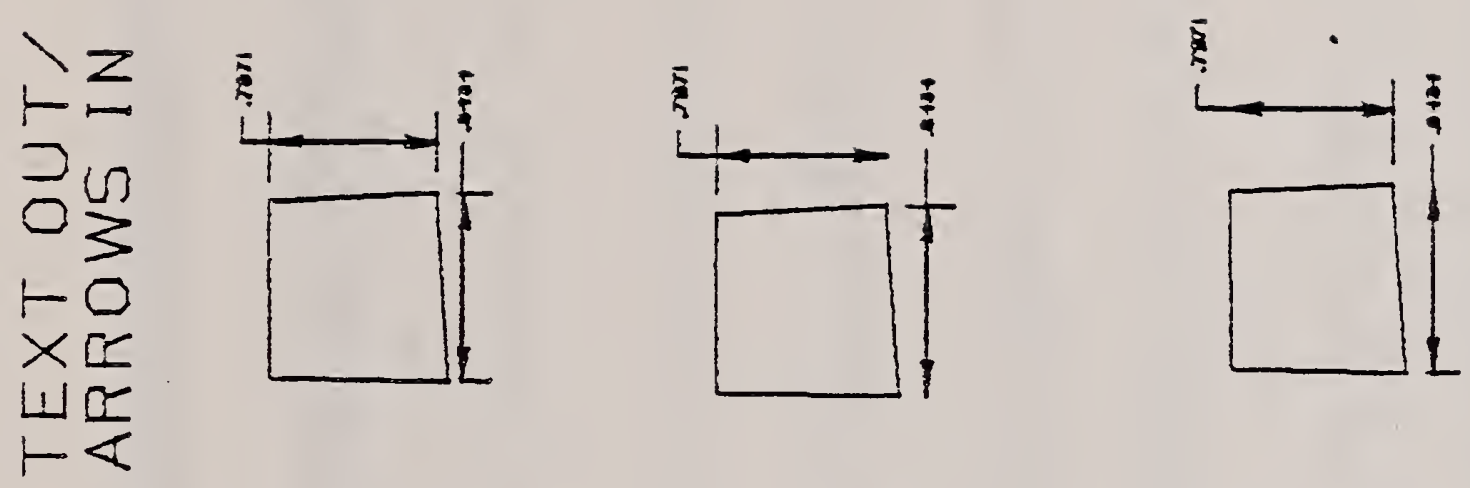


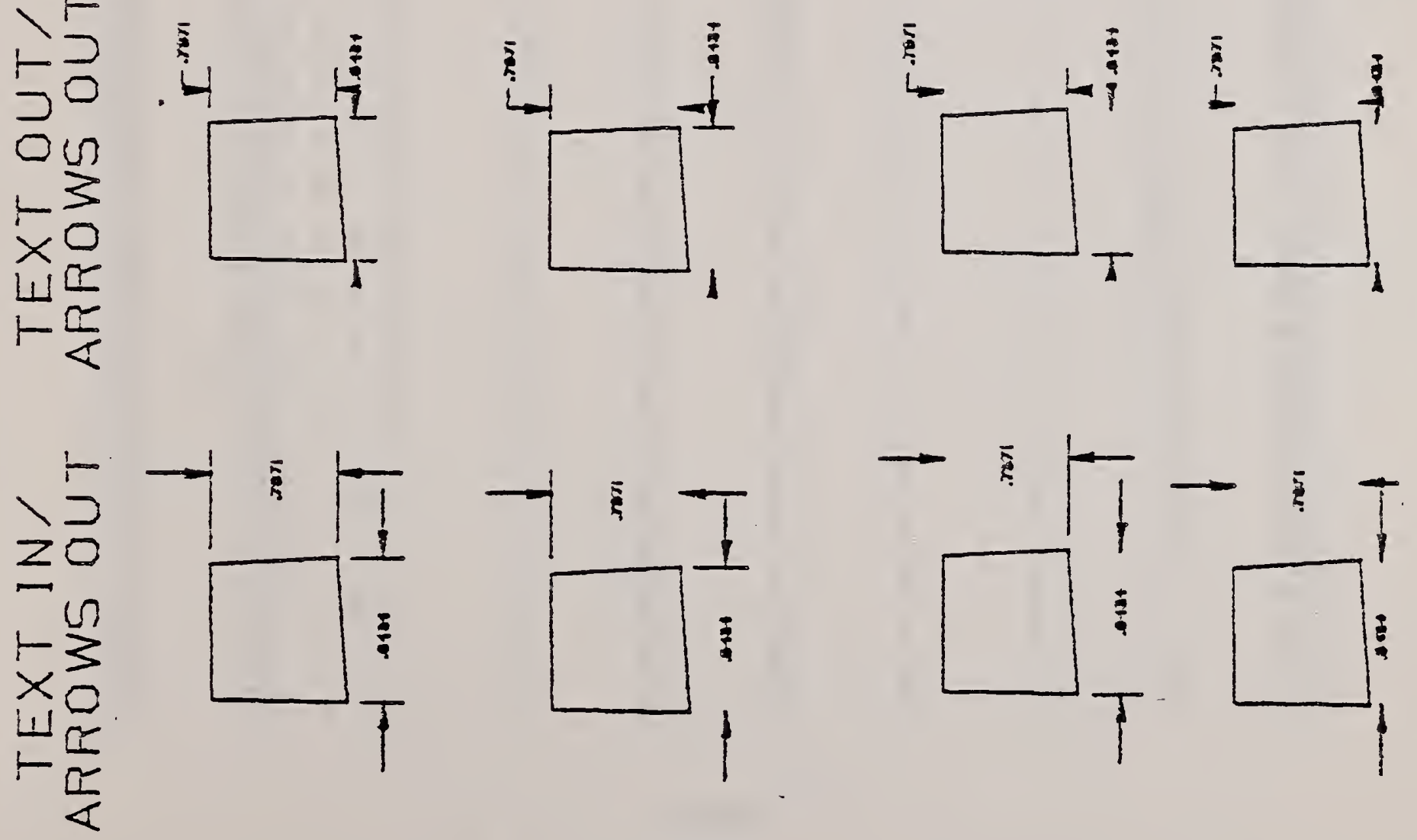


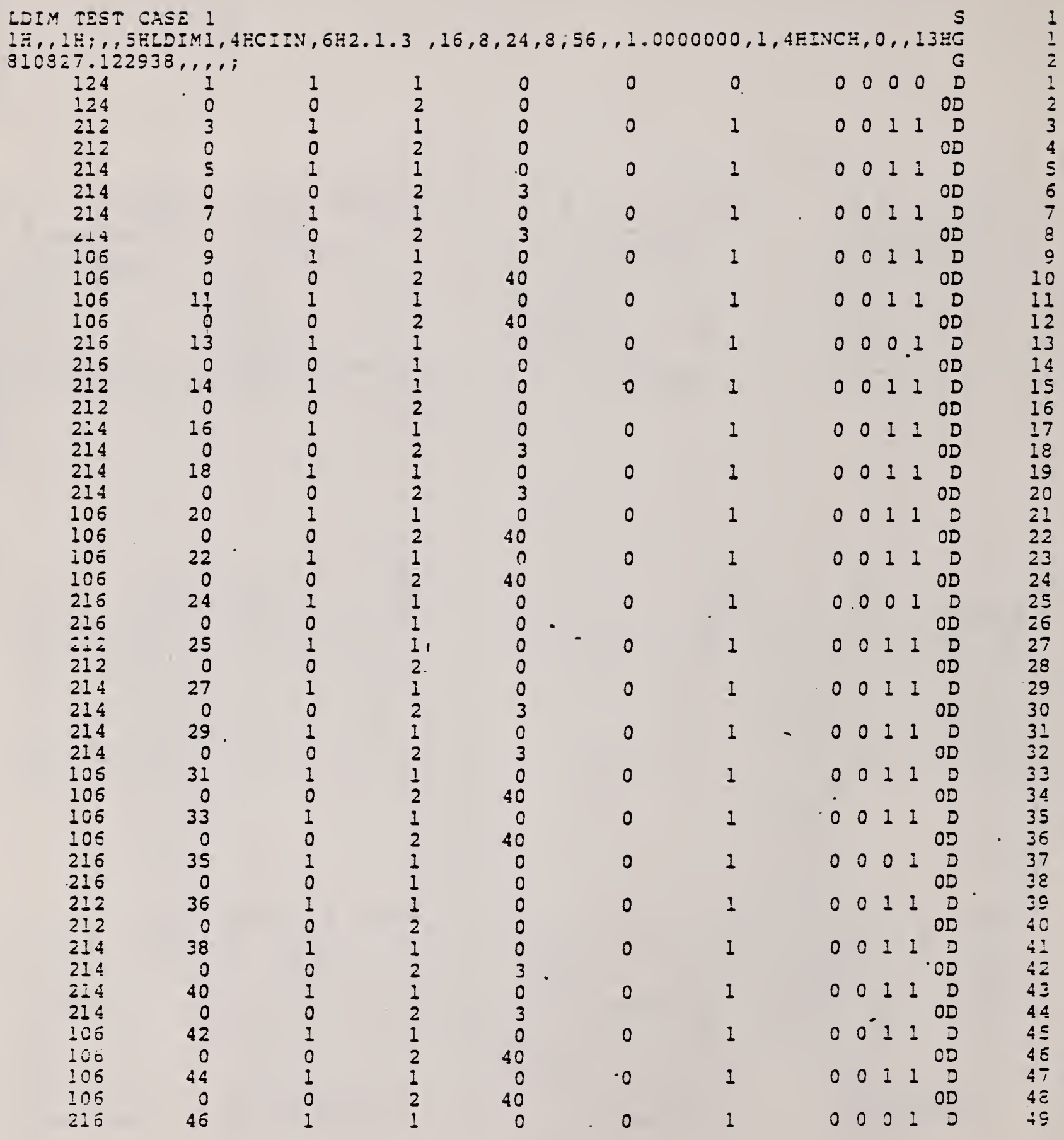




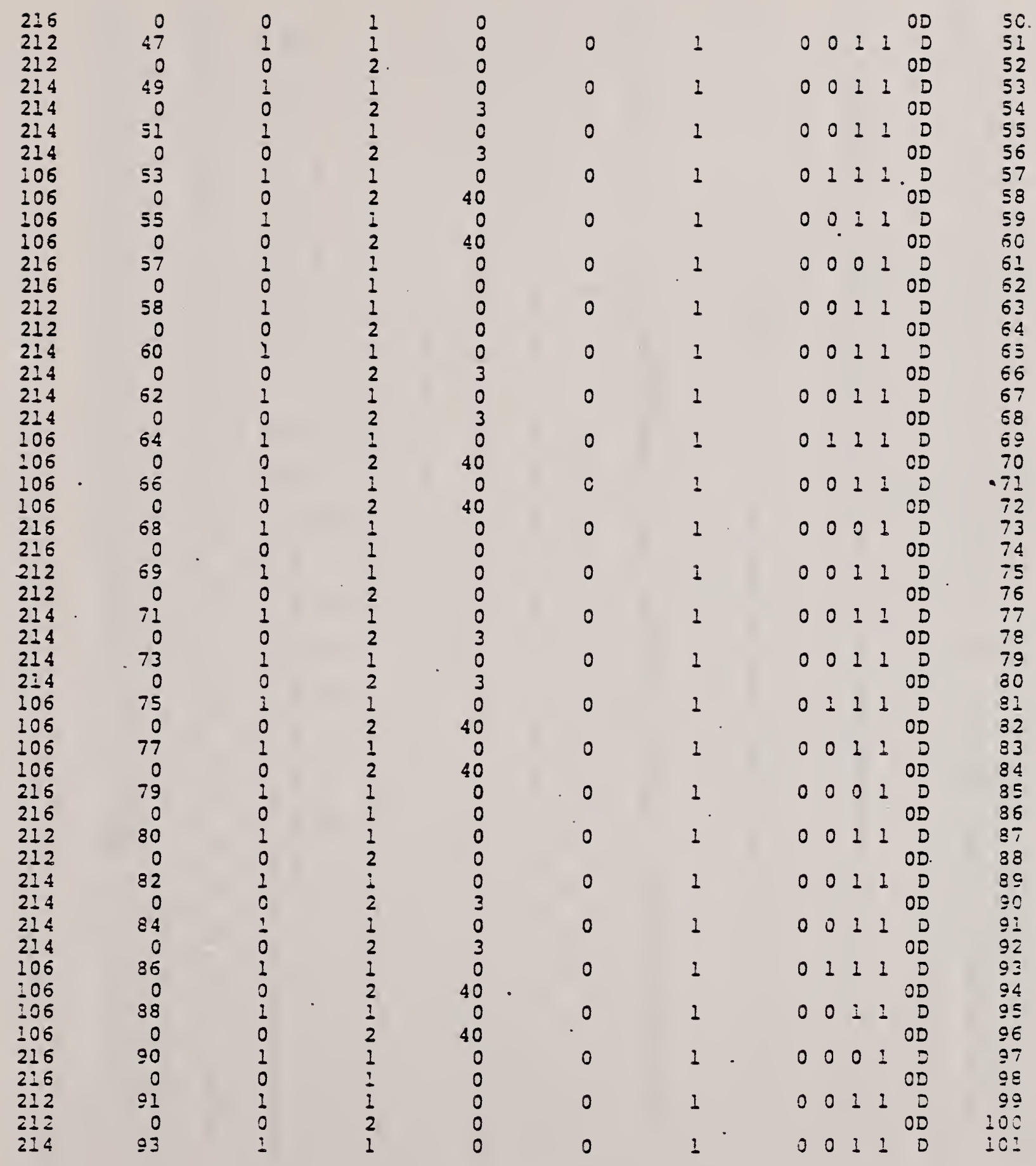




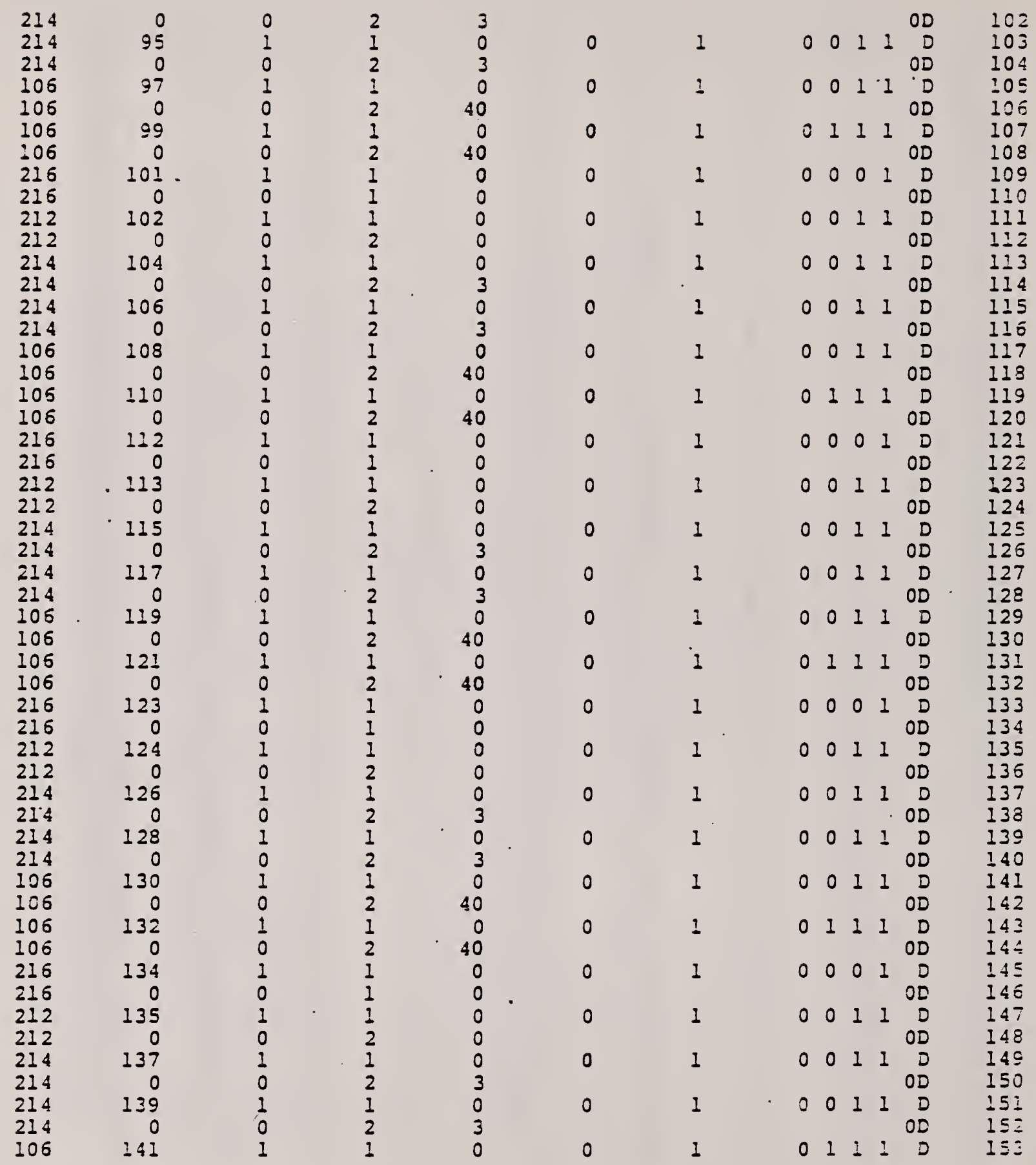




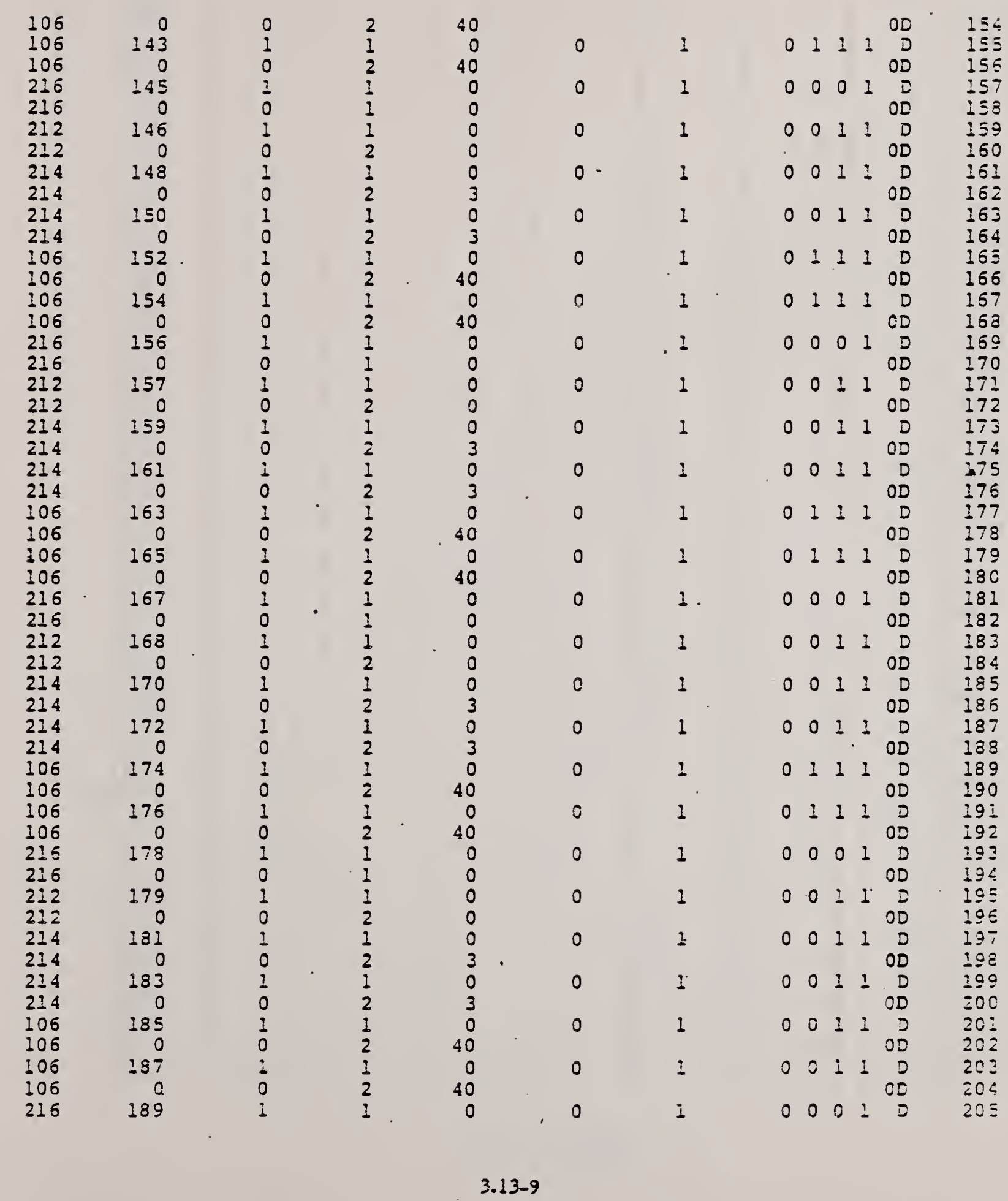




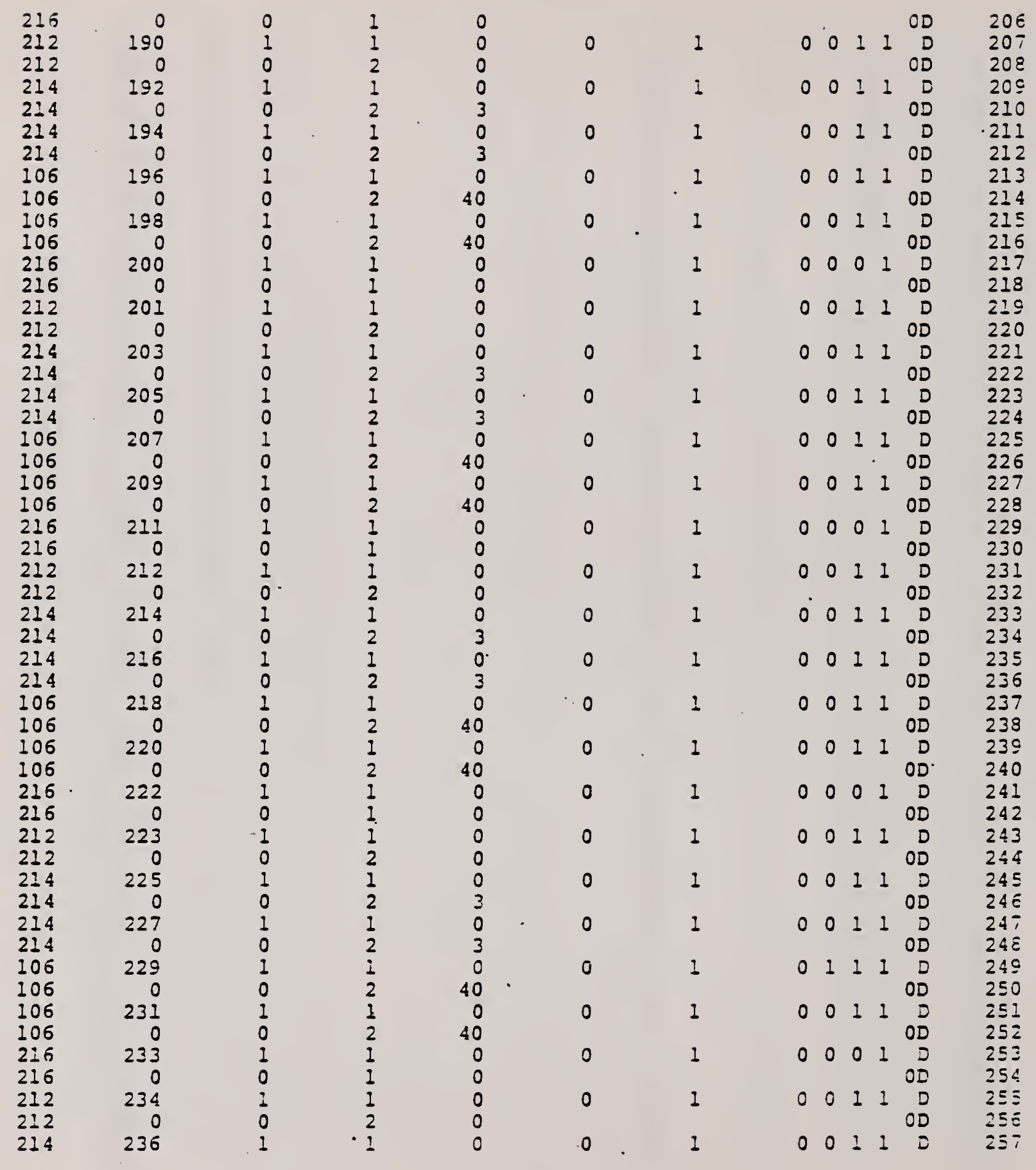




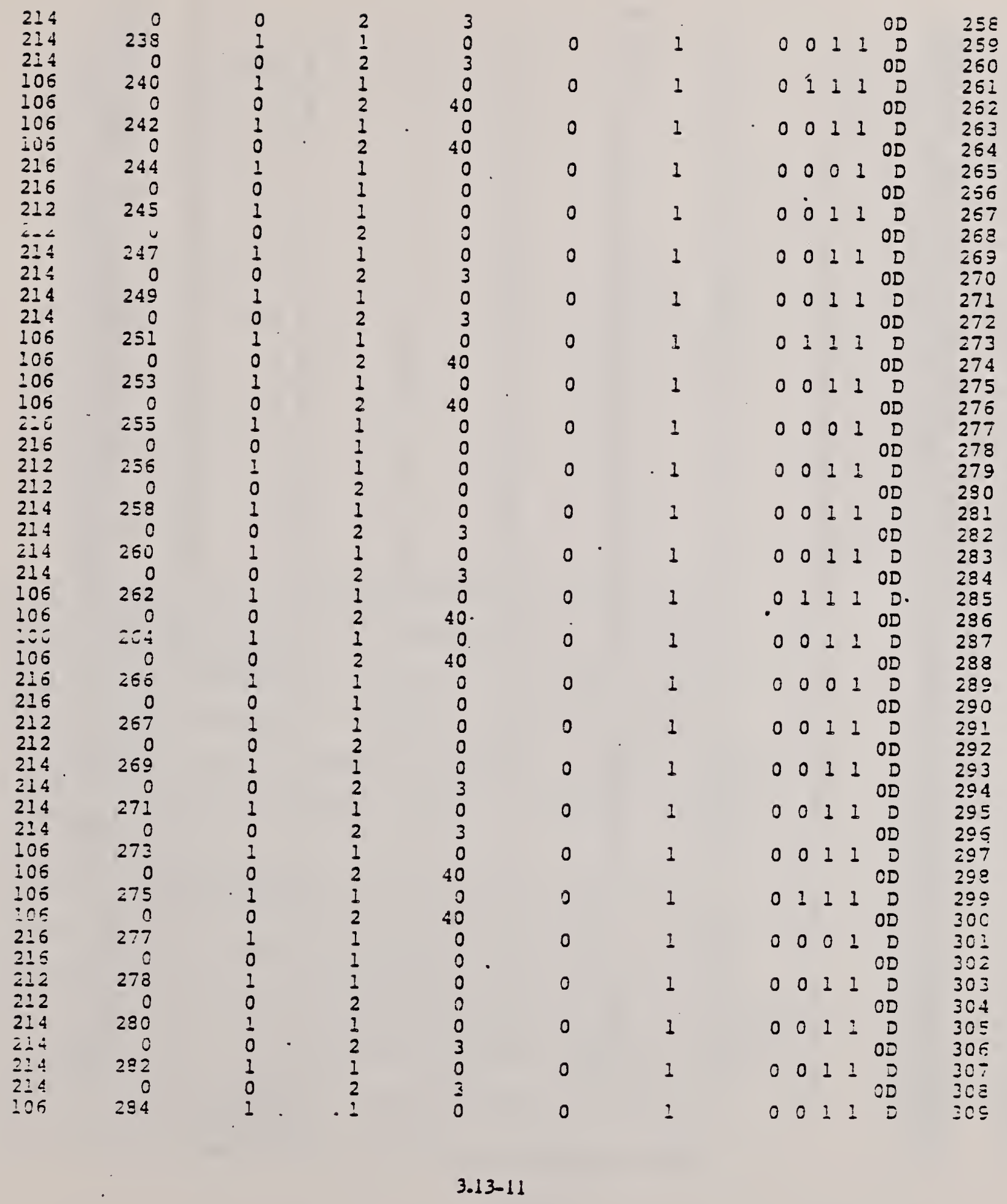




\begin{tabular}{|c|c|c|c|c|c|c|c|c|c|c|c|c|}
\hline 105 & 0 & 0 & 2 & 40 & & & & & & & $O D$ & 310 \\
\hline 105 & 286 & 1 & 1 & 0 & 0 & 1 & 0 & 1 & 1 & 1 & $D$ & 311 \\
\hline 106 & 0 & 0 & 2 & 40 & & & & & & & $O D$ & \\
\hline 216 & 288 & 1 & 1 & 0 & 0 & 1 & 0 & 0 & 0 & 1 & $D$ & 313 \\
\hline 216 & 0 & 0 & 1 & 0 & & & & & & & $0 D$ & $3 \geq 4$ \\
\hline 212 & 289 & 1 & 1 & 0 & 0 & 1 & 0 & 0 & 1 & 1 & $D$ & 315 \\
\hline 212 & 0 & 0 & 2 & 0 & & & & & & & $O D$ & 316 \\
\hline 214 & 291 & 1 & $\frac{1}{2}$ & $\begin{array}{l}0 \\
3\end{array}$ & 0 & 1 & 0 & 0 & 1 & 1 & D & $\begin{array}{l}317 \\
318\end{array}$ \\
\hline $\begin{array}{l}214 \\
=\therefore:\end{array}$ & $=2 ?$ & 1 & 1 & 0 & 0 & 1 & 0 & 0 & 1 & 1 & D & 319 \\
\hline 214 & 0 & 0 & 2 & 3 & & & & & & & $O D$ & 320 \\
\hline 106 & 295 & 1 & 1 & 0 & 0 & 1 & 0 & 0 & 1 & 1 & $D$ & 321 \\
\hline 106 & 0 & 0 & 2 & 40 & & & & & & & $O D$ & 322 \\
\hline 106 & 297 & 1 & 1 & 0 & 0 & 1 & 0 & 1 & 1 & 1 & $D$ & 323 \\
\hline 105 & 0 & 0 & 2 & 40 & & & & & & & $0 D$ & 324 \\
\hline 216 & 299 & 1 & 1 & 0 & 0 & 1 & 0 & 0 & 0 & 1 & $D$ & 325 \\
\hline $\begin{array}{l}216 \\
212\end{array}$ & $\begin{array}{r}0 \\
300\end{array}$ & $\begin{array}{l}0 \\
1\end{array}$ & 1 & 0 & & & & & & & 00 & 326 \\
\hline $\begin{array}{l}212 \\
212\end{array}$ & $\begin{array}{r}300 \\
0\end{array}$ & $\begin{array}{l}1 \\
0\end{array}$ & $\frac{1}{2}$ & $\begin{array}{l}0 \\
0\end{array}$ & 0 & 1 & 0 & 0 & 1 & 1 & D & $\begin{array}{l}327 \\
328\end{array}$ \\
\hline 214 & 302 & 1 & 1 & 0 & 0 & 1 & 0 & 0 & 1 & 1 & D & 329 \\
\hline 214 & 0 & 0 & 2 & 3 & & & & & & & $O D$ & 330 \\
\hline 214 & 304 & 1 & 1 & 0 & 0 & 1 & .0 & 0 & 1 & 1 & $D$ & 331 \\
\hline 214 & 0 & 0 & 2 & 3 & & & & & & & $O D$ & 332 \\
\hline 106 & 306 & 1 & 1 & 0 & 0 & 1 & 0 & 0 & & 1 & 0 & 333 \\
\hline 105 & 0 & 0 & 2 & 40 & & & & & & & OD. & 334 \\
\hline 106 & 308 & 1 & 1 & 0 & 0 & 1 & 0 & 1 & & 1 & $D^{\circ}$ & 335 \\
\hline 106 & 0 & 0 & 2 & 40 & & & & & & & OD & 336 \\
\hline $\begin{array}{l}216 \\
216\end{array}$ & $\begin{array}{r}310 \\
0\end{array}$ & 1 & $\frac{1}{1}$ & $\begin{array}{l}0 \\
0\end{array}$ & 0 & 1 & 0 & 0 & 0 & 1 & D & $\begin{array}{l}337 \\
338\end{array}$ \\
\hline 212 & 311 & 1 & $i$ & 0. & 0 & 1 & 0 & 0 & 1 & 1 & D & 339 \\
\hline 212 & 0 & 0 & 2 & 0 & & & & & & & $O D$ & 340 \\
\hline 214 & 313 & 1 & 1 & 0 & 0 & 1 & 0 & 0 & 1 & 1 & D & 341 \\
\hline 214 & 0 & 0 & 2 & 3 & & & & & & & $O D$ & 342 \\
\hline 214 & 315 & 1 & 1 & 0 & 0 & 1 & 0 & 0 & 1 & 1 & $D$ & 343 \\
\hline $2 \geq 4$ & 0 & 0 & 2 & 3 & & & & & & & $O D$ & 344 \\
\hline 105 & 317 & 1 & 1 & 0 & 0 & 1 & 0 & 1 & 1 & 1 & $D$ & 345 \\
\hline 106 & 0 & 0 & 2 & 40 & & & & & & & $O D$ & 346 \\
\hline 100 & 319 & 1 & 1 & 0 & 0 & 1 & 0 & 1 & 1 & 1 & D & $\begin{array}{l}347 \\
348\end{array}$ \\
\hline 106 & 0 & 0 & 2 & 40 & & & & & & 7 & OD & $\begin{array}{l}348 \\
349\end{array}$ \\
\hline $\begin{array}{l}216 \\
216\end{array}$ & $\begin{array}{r}321 \\
0\end{array}$ & $\begin{array}{l}1 \\
0\end{array}$ & $\frac{1}{1}$ & $\begin{array}{l}0 \\
0\end{array}$ & 0 & 1 & 0 & 0 & 0 & 1 & $O D$ & $35 c$ \\
\hline 212 & 322 & 1 & 1 & 0 & 0 & 1 & 0 & 0 & 1 & 1 & D & 351 \\
\hline 212 & 0 & 0 & 2 & 0 & & & & & & & D & 352 \\
\hline 214 & 324 & 1 & 1 & 0 & 0 & 1 & 0 & 0 & 1 & 1 & D & $3 \leq 3$ \\
\hline 214 & 0 & 0 & 2 & 3 & & & & & & & OD & 354 \\
\hline $2 \div 4$ & 326 & 1 & 1 & 0 & 0 & 1 & 0 & 0 & 1 & 1 & D & $\begin{array}{l}355 \\
356\end{array}$ \\
\hline$=\div 4$ & $=-0$ & 0 & 2 & 3 & & & 0 & 1 & 1 & I & $\begin{array}{r}O D \\
D\end{array}$ & $\begin{array}{l}3=6 \\
357\end{array}$ \\
\hline 100 & 328 & 1 & $\frac{1}{2}$ & $\begin{array}{r}0 \\
40\end{array}$ & 0 & 1 & 0 & & & & $O D$ & 352 \\
\hline 105 & 330 & 1 & 1 & 0 & 0 & 1 & 0 & 1 & 1 & 1 & D & 350 \\
\hline 106 & 0 & 0 & 2 & 40 & & & & & & & OD & 360 \\
\hline $2: \varepsilon$ & 332 & 1 & 1 & 0 & 0 & 1 & 0 & 0 & 0 & : & D & $36:$ \\
\hline
\end{tabular}




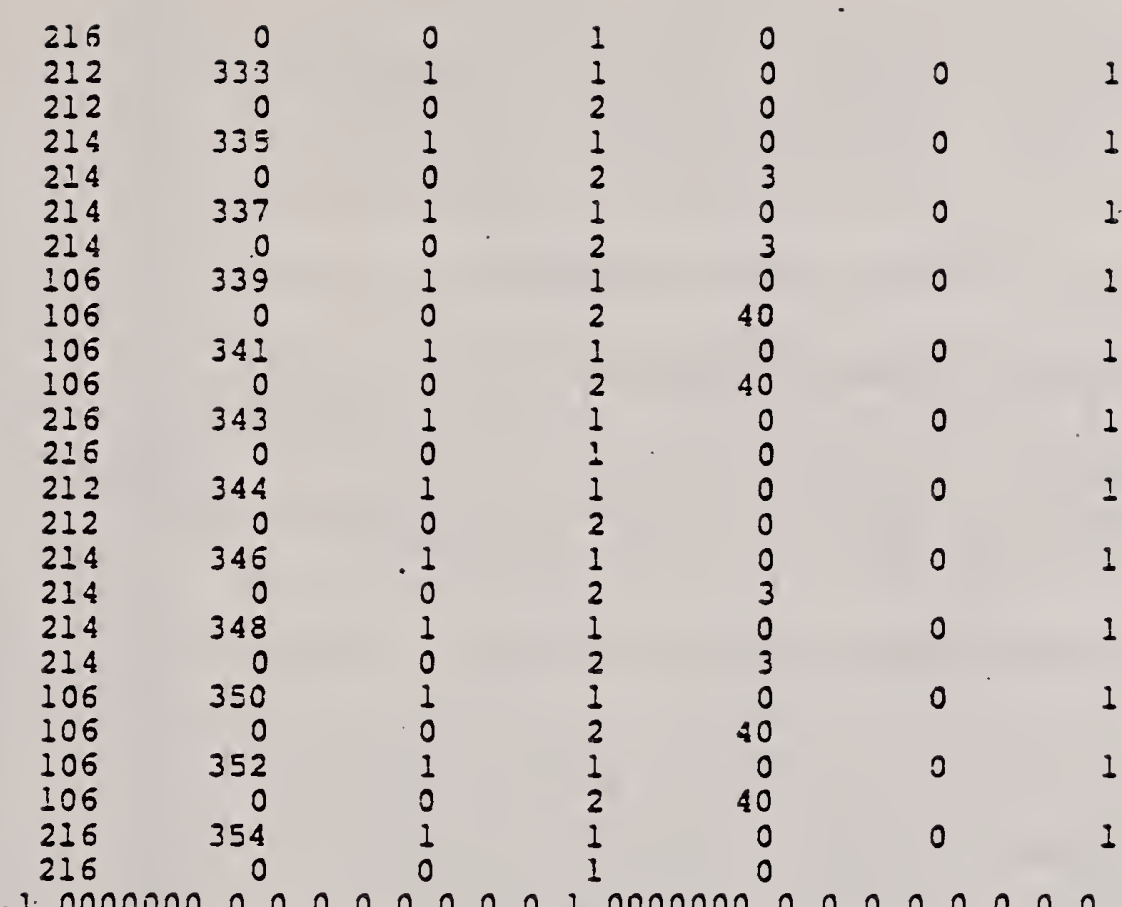

$124,1.0000000,0.0,0.0,0.0,0.0,1.0000000,0.0,0.0,0.0,0.0$, $1.0000000,0.0,0,0$;

$212,1,5, .1606400, .0543000,1,1.5707963,0.0,0,0,-.3310501$, $3.7099235,0.0,54.8434,0,0$;

$214,1, .1500000, .0500000,0.0,-.6234555,3.7370737,-.3853501$, $3.7370736,0,0$;

$214,1, .1500000, .0500000,0.0, .2199411,3.7370734, .0381899^{\prime}$, $3.7370735,0,0$

$106,1,3,0.0,-.6234555,3.8810318,-.6234555,3.8810318,-.6234555$, $3.6120737,0,0$;

$106,1,3,0.0, .2199411,3.9435048, .2199411,3.9435048, .2199411$, $3.6120734,0,0$;

$216,3,5,7,9,11,0,0$;

$212,1,5, .1606400, .0543000,1,1.5707963,0.0,0,0,2.3729501$,

$3.7739236,0.0,5 \mathrm{z} .8434,0.0$;

$214,1, .1500000, .0500000,0.0,2.1125445,3.3010736,1.9064388$, $3.8010736,0,0$;

$214,1, .1500000, .0500000,0.0,2.9559417,3.80: 0736,3.1596933$, $3.8010736,0,0$;

$106,1,3,0.0,2.1125445,3.9370313,2.1125445 ; 3.9370313,2.1125445$, $3.6760736,0,0$;

$106,1,3.0 .0,2.9559417,3.9995050,2.9559417,3.9995050,2.9559417$, $3.6760736,0,0$;

$216,15,17,19,21,23,0,0$;

$212,1,5, .1606400, .0543000,1,1.5707953,0.0,0,0,5.7529502$,

$3.9499242,0.0,5 \mathrm{H} .3434,0,0$;

$214,1, .1500000, .0500000,0.0,4.6925449,3.9770741,4.5652793$,

$\begin{array}{lllll}0 & 0 & 1 & 0 D & 362 \\ 0 & 363\end{array}$

364

355

366

367

368

369

370

371

372

373

374

375

376

377

378

379

380

381

382

383

384

$\begin{array}{lllllll}0 & 0 & 0 & 1 & D & 385 \\ O D & & 386\end{array}$

$\begin{array}{rr}\text { OD } & 386 \\ \text { IP } & 1 \\ \text { IP } & 2\end{array}$

$3 \mathrm{P}$

38

$5 P$

$5 P$

$7 P$

$7 P$

$9 P$

QP

11P

11P

$13 P$

$15 \mathrm{P}$

$15 P$

172

172

$19 P$

$19 P$

21?

21?

$23 P$

232

25?

$27 ?$

27?

29 ? 
$3.9770741,0.0$;

$214,2, .1500000, .0500000,0.0,5.5359416,3.9770741,5.5900502$, $3.9770741,5.6986499,3.9770744,0,0$;

$106,1,3,0.0,4.6925449,3.9450312,4.6925449,3.9450312,4.6925449$, $3.8520741,0,0$;

$106,1,3,0.0,5.5359416,4.0075045,5.53 .59416,4.0075045,5.5359416$, $3.8520741,0,0$;

$216,27,29,31,33,35,0,0 ;$

$212,1,5, .1606400,00543000,1,1.5707963,0.0,0,0,3.5289497$, $3.9659240,0.0,5 \pi .8434,0,0$;

$214,3, .1500000, .0500000,0.0,7.4125433,3.9930739,7.8342419$, $3.9930739,7.8342419,3.9930742,8.4746494,3.9930742,0,0$;

$214,1, .1500000, .0500000,0.0,8.2559404,3.9930739,7.8342419$, $3.9930739,0,0 ;$

$106,1,3,0.0,7.4125433,3.9690323,7.4125433,3.9690323,7.4125433$, $3.8680739,0,0$;

$106,1,3,0.0,8.2559404,4.0315061,8.2559404,4.0315061,8.2559404$, $3.8680739,0,0$;

$216,39,41,43,45,47,0,0$;

$212,1,5, .1506400, .0543000,1,1.5707963,0.0,0,0,0.3790502$,

$1.6099244,0.0,54.8434,0,0$;

$214,1, .1500000, .0500000,0.0,-.6794549,1.6370744,-.4333502$,

$1.6370744,0,0$;

$214,1, .1500000,00500000,0.0, .1639414,1.6370744,-.0098102$,

$1.6370744,0,0$;

$106,1,3,0.0,-.6794549,1.8035065,-.6794549,1.8035065,-.6794549$, $1.5120744,0,0$;

$106,1,3,0.0, .1639414,1.8035065, .1639414,1.8035065, .1639414$,

$1.5120744,0,0$;

$216,51,53,55,57,59,0,0 ;$

$212,1,5, .1606400, .0543000,1,1.5707963,0.0,0,0,2.3489499$,

$1.6419244,0.0,54.8434,0,0$;

$214,1, .1500000, .0500000,0.0,2.0565453,1.6690743,1.8184404$, $1.6690743,0,0$;

$214,1, .1500000, .0500000,0.0,2.8999419,1.6690743,3.0816940$,

$1.6690743,0,0$;

$106,1,3,0.0,2.0565453,1.8595064,2.0565453,1.8595064,2.0565453$, $1.5440743,0,0$;

$106,1,3,0.0,2.8999419,1.8595064,2.8999419,1.8595064,2.8999419$, $1.5440743,0,0$;

$216,63,65,67,69,71,0,0$;

$212,1,5, .1606400, .0543000,1,1.5707963,0.0,0,0,5.8249497$, $1.7299243,0.0,53.8434,0,0$;

$214,1, .1500000, .0500000,0.0,4.6365452,1.7570742,4.5092796$, $1.7570742,0,0$;

$214,2, .1500000, .0500000,0.0,5.4799418,1.7570742,5.6620498$,

$1.7570742,5.7706494,1.7570742,0,0$;

$105,1,3,0.0,4.6365452,1.3675066,4.6365452,1.8675066,4.6365452$, $1.6320742,0,0$;

$\$ 06,1,3,0.0,5.4799418,1.3675066,5.4799418,1.8675066,5.4799418$, $1.6320742,0,0$;

$216,75,77,79,81,83,0,0$;

$\begin{array}{ll}29 P & 28 \\ 31 P & 29 \\ 31 P & 3 C \\ 33 P & 31 \\ 33 P & 32 \\ 35 P & 33 \\ 35 P & 34 \\ 37 P & 35 \\ 39 P & 36 \\ 39 P & 37 \\ 41 P & 38 \\ 41 P & 39 \\ 43 P & 40 \\ 43 P & 41 \\ 45 P & 42 \\ 45 P & 43 \\ 47 P & 44 \\ 47 P & 45 \\ 49 P & 46 \\ 51 P & 47 \\ 51 P & 48 \\ 53 P & 49 \\ 53 P & 50 \\ 55 P & 51 \\ 55 P & 52 \\ 57 P & 53 \\ 57 P & 54 \\ 59 P & 55 \\ 59 P & 56 \\ 61 P & 57 \\ 63 P & 58 \\ 63 P & 59 \\ 65 P & 60 \\ 65 P & 61 \\ 67 P & 62 \\ 67 P & 63 \\ 69 P & 64 \\ 83 P & 79 \\ 89 P & 79 \\ 71 P & 65 \\ 71 P & 67 \\ 73 P & 68 \\ 75 P & 69 \\ 75 P & 7 C \\ 77 P & 71 \\ 77 P & 79 \\ 79 P & 79 \\ 79 P & 74 \\ 81 P & 75 \\ 89 & 79 \\ 89 & \end{array}$


$212,1,5, .1506400, .0543000,1,1.5707953,0.0,0,0,8.5049495$, $1.7699242,0.0,54.8434,0,0$;

$214,3, .1500000, .0500000,0.0,7.3565435,1.7970741,7.7782421$, $1.7970741,7.7782421,1.7970741,8.4506493,1.7970741,0,0$;

$214,1, .1500000, .0500000,0.0,8.1999407 ; 1.7970741,7.7782421$, $1.7970741,0,0$

$106,1,3,0.0,7.3565435,1.8915074,7.3565435,1.8915074,7.3565435$, $1.6720741,0,0$;

$106,1,3,0.0,8.1999407,1.8915074,8.1999407,1.8915074,8.1999407$, $1.6720741,0,0$;

$216,87,89,91,93,95,0,0$;

$212,1,5, .1606400, .0543000,1,1.5707963,0.0,0,0,-.2470502$,

$-.8980753,0.0,54.8434,0,0$;

$214,1, .1500000, .0500000,0.0,-.5714565,-.8709253,-.3013502$,

$-.8709253,0,0$;

$214,1, .1500000, .0500000,0.0, .2719398,-.8709253, .1221898$,

$-.8709253,0,0$;

$106,1,3,0.0,-.5714565,-.7029657,-.5714565,-.7029657,-.5714565$, $-.9959253,0,0$;

$106,1,3,0.0, .2719398,-.7029657, .2719398,-.7029657, .2719398$,

-.9959253,0,0;

$216,99,101,103,105,107,0,0 ;$

$212,1,5, .1606400, .0543000,1,1.5707963,0.0,0,0,2.5129499$,

$-.3500753,0.0,54.8434,0,0$.

$214,1, .1500000, .0500000,0.0,2.1645436,-.8229253,1.8704371$,

$-.8229253,0,0$;

$214,1, .1500000, .0500000,0.0,3.0079403,-.8229253,3.1336907$,

$-.8229253,0,0$;

$106,1,3,0.0,2.1645436,-.6469661,2.1645436,-.6459661,2.1645436$, $.9479253,0,0$;

$106,1,3,0.0,3.0079403,-.6469661,3.0079403,-.6469661,3.0079403$, $-9473253,0,0$;

$216,111,113,115,117,119,0,0$;

$212,1,5, .1606400, .0543000,1,1.5707963,0.0,0,0,5.7969503$,

.7860753,0.0,58.8434,0,0:

$214,1, .1500000, .0500000,0.0,4.7445436,-.7589253,4.6172780$,

$-.7589253,0,0$;

$214,2, .1500000, .0500000,0.0,5.5879402,-.7589254,5.6340504$,

$.7589254,5.7426500,-.7589253,0,0$;

$106,1,3,0.0,4.7445436,-.6389657,4.7445436,-.6389657,4.7445436$, $-.8939253,0,0$;

$106,1,3,0.0,5.5879402,-.6389657,5.5879402,-.5389557,5.5879402$, $-.8839254,0,0$;

$216,123,125,127,129,131,0,0$;

$212,1,5, .1606400, .0543000,1,1.5707963,0.0,0,0,8.5329494$,

$-.6740752,0.0,54.8434,0,0$;

$214,1, .1500000, .0500000,0.0,7.4645410,-.6469253,7.8862398$,

$-.6469253,0,0$

$214,3, .1500000, .0500000,0.0,8.3079386,-.6469253,7.8862398$,

$-.6469253,7.8862398,-.6469253,3.4786491,-.6469253,0,0$;

$106,1,3,0.0,7.4645410,-.6149648,7.4645410,-.6149648,7.4645410$, $-.7719253,0,0$; 
$106,1,3,0.0,8.3079386,-.6149648,8.3079386,-.6149648,3.3079386$, $-.7719253,0,0$;

$216,135,137,139,141,143,0,0 ;$

$212,1,5, .1606400, .0543000,1,1.5707963,0.0,0,0,-.3530502$,

$-2.5 \equiv 00751,0.0,58.3434,0,0$;

$214,3, .1500000, .0500000,0.0,-.6474565,-2.5229250,-.2257583$,

$-2.5229250,-.2257583,-2.5229251,-.4173502,-2.5229251,0,0$;

$214,1, .1500000, .0500000,0.0, .1959399,-2.5229250,-.2257583$,

$-2.5229250,0,0$;

$:=\leq, \vdots, \Sigma, 0.2,-.0474565,-1.9916750,-.6474565,-1.0916750,-.6474565$,

$-2,6479250,0,0$;

$105,1,3,0.0, .1959399,-1.9916750, .1959399,-1.9916750, .1959399$,

$-2.6479250,0,0$ :

$216,147,143,151,153,155,0,0$;

$212,1,5, .1606400, .0543000,1,1.5707963,0.0,0.0,2.3489499$,

$-2.50207 .52,0.0,5 \% .8434,0,0$;

$214,1, .1500000, .0500000,0.0,2.0885434,-2.4749253,1.8824368$, $-2.4749253,0,0$;

$214,1, .2500000,00500000,0.0,2.9319401,-2.4749253,3.1456903$,

$-2.4749253,0,0$;

$106,1,3,0.0,2.0885434,-1.9436753,2.0885434,-1.9436753,2.0885434$, $-2.5999253,0,0$;

$106,1,3,0.0,2.9319401,-1.9436753,2.9319401,-1.9436753,2.9319401$, $-2.5999253,0,0$;

$216,259,251,263,165,167,0,0$;

$212,1,5, .1606400, .0543000,1,1.5707953,0.0 ; 0,0,5.6409497$,

$-2.4700751,0.0,5 \mathrm{H} .8434,0,0$ :

$214,1, .5500000, .0500000,0.0,4.6685438,-2.4429251,4.5412782$, -?. $4.29252,0,0$;

$214,2, .1500000, .0500000,0.0,5 . \equiv 119405,-2.4429251,5.5458312$,

$-2.4429251,5.5866494,-2.4429252,0,0$;

$106,1,3,0.0,4.5685438,-1.9116751,4.6685438,-1.9116751,4.6685438$, $-2.5679251,0,0$ :

$106,1,3,0.0,5.5119405,-1.9116751,5.5119405,-1.9116751,5.5119405$, $-2.5679251,0,0$;

$2: 6,171,173,175,177,179,0,0 ;$

$212,1,5, .1606400, .0543000,1,1.5707963,0.0,0,0,8.4459953$,

$-2.4373329,0.0,5$ i. $8434,0,0$;

$214,3, .1500000, .0500000,0.0,7.3885422,-2.4101831,7.8102407$,

$-2.4101831,7.8102407,-2.4101830,8.3916950,-2.4101830,0,0$;

$214,1, .1500000, .0500000,0.0,3.2319393,-2.4101831,7.8102407$,

$-2.4101831,0,0$

$100,1,3,0.0,7.3885422,-1.8789331,7.3885422,-1.8789331,7.3885422$,

$-2.5351831,0,0$;

$106,1,3,0.0,8.2319393,-1.8789331,8.2319393 ;-1.8789331,8.2319393$,

$-2.535 i 831,0,0$;

$216,183,135,187,189,191,0,0 ;$

$\because:, \ldots, \ldots=25300, .0543000,1,1.5707963,0.0,0,0, .5227866$,

$4.4041305,0.0,58.7971,0,0$;

$224,1, .1500000, .0500000,0.0, .6802565,4.0372548, .6802565$,

$4.3498 \leq 06,0,0 ;$

$\therefore \therefore, \ldots \ldots=0000, .0500000,0.0, .6802565,4.8343368, .5802565$,

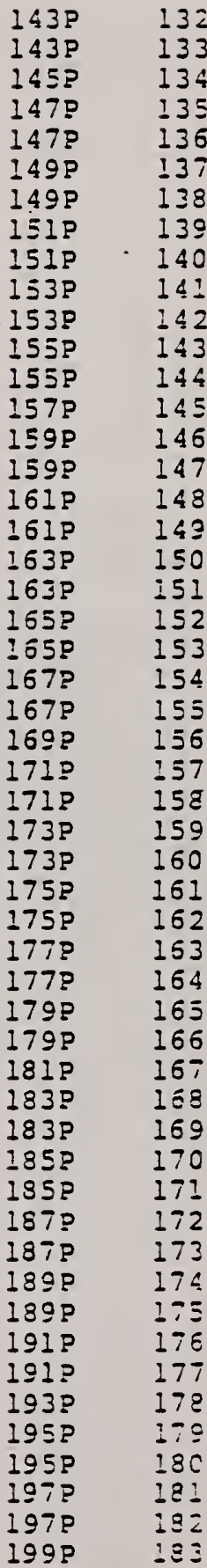


$4.5127311,0,0$;

$199 ? \quad 124$

$106,1,3,0.0, .3136918,4.0372548, .3136918,4.0372548, .8052565$, $4.0372548,0,0$;

$106,1,3,0.0, .2759593,4.8343368, .2759593,4.8343368, .8052565$, $4.8343368,0,0$;

$216,195,197,199,201,203,0,0 ;$

$212,1,5, .1226300, .0543000,1,1.5707963,0.0,0,0,3.2907867$,

$4.4521308,0.0,53.7971,0,0 ;$

$214,1, .1500000, .0500000,0.0,3.4482570,4.0932550,3.4482570$,

$3.7886791,0,0$;

$214,1, .1500000, .0500000,0.0,3.4482570,4.8903365,3.4482570$,

$5.2199415,0,0$;

$106,1,3,0.0,3.0496922,4.0932550,3.0496922,4.0932550,3.5732570$,

$4.0932550,0,0$;

$106,1,3,0.0,3.0119591,4.8903365,3.0119591,4.8903365,3.5732570$,

$4.8903365,0,0$;

$216,207,209,211,213,215,0,0$;

$212,1,5, .1226300, .0543000,1,1.5707963,0.0,0,0,5.8556762$,

$5.0316863,0.0,53.7971,0,0 ;$

$214,1, .1500000, .0500000,0.0,5.6927762,4.1012545,5.6927762$,

$3.9739889,0,0$

$214,2, .1500000, .0500000,0.0,5.6927762,4.8983369,5.6927762$,

$5.0588365,5.8013759,5.0588365,0,0 ;$

$106,1,3,0.0,5.6296926,4.1012545,5.6296926,4.1012545,5.8177762$, $4.1012545,0,0$ :

$106,1,3,0.0,5.5919585,4.8983369,5.5919585,4.8983369,5.3177762$, $4.8983369,0,0$;

$2 \pm 5,219,221,223,225,227,0,0$;

$212,1,5, .1226300, .0543000,1,1.5707963,0.0,0,0,8.6423426$,

$5.0227971,0.0,51.7971,0,0$;

$214,3, .1500000, .0500000,0.0,8.4794426,4.1252561,8.4794425$,

$4.5237970,8.4794426,5.0499473,8.5880423,5.0499473,0,0$;

$214,1, .1500000, .0500000,0.0,8.4794426,4.9223380,8.4794426$, $4.5237970,0,0 ;$

$106,1,3,0.0,8.3496914,4.1252561,8.3496914,4.1252561,8.6044426$, $4.1252561,0,0$;

$106,1, \bar{z}, 0.0,3.3119574,4.9223380,8.3119574,4.9223380,8.6044426$, $4.9223380,0,0$;

$216,231,233,235,237,239,0,0$;

$212,1,5, .1226300, .0543000,1,1.5707963,0.0,0,0, .5067868$,

$2.2739091,0.0,51.7971,0.0 ;$

$214,1, .1500000, .0500000,0.0, .6642568,1.8972565, .6642568$,

$2.2196094,0,0$;

$214, \dot{1}, 1500000, .0500000,0.0, .6542558,2.6943388, .6542568$,

$2.3825092,0,0$;

$105,1,3,0.0, .2199603,1.8972565, .2199603,1.8972565, .7892568$, $1.8972565,0,0$

$106,1,3,0.0, .2199603,2.6943388, .2199603,2.6943388, .7892568$,

$2.6943338,0,0$;

$216,543,245,247,249,251,0,0$

$212,1,5, .1226300, .0543000,1,1.5707963,0.0,0,0,3.1734543$,

$2.3183541,0.0,5 \mathrm{H} .7971,0,0$

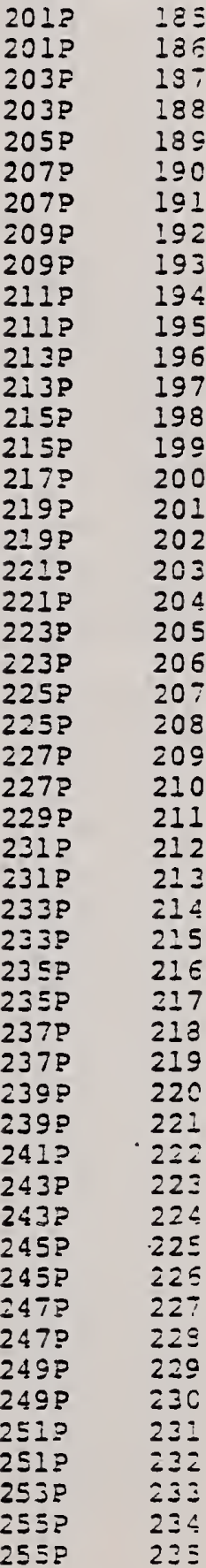

$3.13-17$. 
$214,1, .1500000, .0500000,0.0,3.3309245,1.9532561,3.3309245$,

$1.6 \div 24580,0,0$;

$214,1, .1500000, .0500000,0.0,3.3309245,2.7503386,3.3309245$,

$3.0737228,0,0$;

$106,1,3,0.0,2.9559598,1.9532561,2.9559598,1.9532561,3.4559245$, 1. $9532561,0,0$;

$105,1,3,0.0,2.9559598,2.7503386,2.9559598,2.7503386,3.4559245$, $2.7503386,0,0$;

$215,255,257,259,251,263,0,0$;

$212.1,5, .1226300, .0543000,1,1.5707963,0.0,0,0,5.7490096$, $2.9850202,0.0,54.7971,0,0$;

$214,1, .1500000, .0500000,0.0,5.5861096,1.9612564,5.5861096$, ¿. $8337908,0,0$;

$214,2, .1500000, .0500000,0.0,5.5861096,2.7583389,5.5861096$, $3.0121703,5.6947093,3.0121703,0,0$;

$106,1,3,0.0,5.5359597,1.9612564,5.5359597,1.9612564,5.7111096$, $1.9612564,0,0$;

$106,1,3,0.0,5.5359597,2.7583389,5.5359597,2.7583389,5.7111096$, $2.7583389,0,0$;

$216,267,269,271,273,275,0,0$;

$212,1,5, .1226300, .0543000,1,1.5707963,0.0,0,0,8.5890093$,

$2.93168 .69,0.0,5 \% .7972,0,0$;

$214,3, .1500000,00500000,0.0,8.4261093,1.9852574,8.4261093$,

$2.3837985,8.4251093,2.9588370,8.5347090,2.9588370,0,0$;

$21\{, 1, .1500000, .0500000,0.0,8.4261093,2.7823396,8.4261093$, $2.3837985,0,0$;

$106,1,3,0.0,8.2559586,1.9852574,8.2559586,1.9852574,8.5511093$, $1.9852574,0,0$;

$106,1,3,0.0,3.2539586,2.7823396,8.2559586,2.7823396,8.5511093$, 2. $1023396,0,0$;

$216,279,281,283,285,287,0,0$;

$212,1,5, .1226300, .0543000,1,1.5707963,0.0,0,0, .6334534$,

$-.1283128,0.0,57.7971,0,0$;

$214,1, .1500000, .0500000,0.0, .7909234,-.5467421, .7909234$,

-.1826127,0,0;

$214,1 \ldots 1500000, .0500000,0.0, .7909234, .2503400, .7909234$,

$-.0197127,0,0$;

$106,1,3,0.0, .3656907,-.5467421, .3656907,-.5467421, .9159234$,

$-.5467421,0,0$;

$105,1,3,0.0, .3656907, .2503400, .3656907, .2503400, .9159234$, $.2503400,0,0$;

$216,291,293,295,297,299,0,0$;

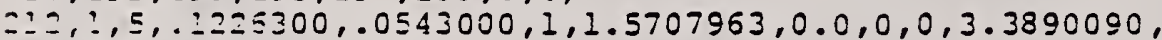

$-. \$ 283129,0.0,5 \% .7971,0,0$;

$=24,2, .2500000, .0500000,0.0,3.5464792,-.4907423,3.5464792$,

$-.7988717,0,0$;

$214,1, .1500000,00500000,0.0,3.5464792, .3063399,3.5464792$,

$.8323927,0,0$;

I $0=1,1,3,0.0,3.1016908,-.4907423,3.1016908,-.4907423,3.6714792$,

- $4327423,0,0$;

$\therefore$, $:, 3,0.0,3.1016908, .3063399,3.1016908, .3063399,3.6714792$,

2ก $52309,0,0$

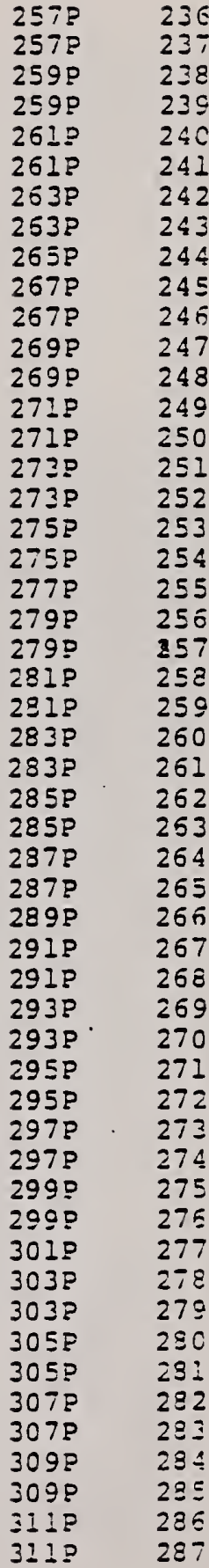


$215,303,305,307,309,311,0,0$;

$212,1,5, .1226300, .0543000,1,1.5707963,0.0,0,0,5.8 .578987$, $.5027984,0.0,5 \pi, 7971,0,0$;

$214,1, .1500000, .0500000,0.0,5.6949987,-.4827421,5.6949987$, $-6100077,0,0$;

$214,2, .1500000, .0500000,0.0,5.5949987, .3143407,5.6949987$, $.5299483,5.8035984, .5299483,0,0$;

१ง $\varepsilon_{1} 1,3,0.0,5.6816912,-.4827421,5.6816912,-.4827421,5.8199987$,

$-. \$ 827421,0,0$;

$\because A=2,0.5 .5816912, .3143407,5.6816912, .3143407,5.8199987$, $.3143407,0,0$;

$216,315,317,319,321,323,0,0$

$212,1,5, .1226300, .0543000,1,1.5707963,0.0,0,0,8.8045654$, $4672429,0.0,5 \pi .7971,0,0$;

$214,3, .1500000, .0500000,0.0,8.6416655,-.4587413,8.6416655$,

$-.0601999,8.6416655, .4943928,8.7502651, .4943928,0,0$;

$214,1, .1500000, .0500000,0.0,8.6416655, .3383414,8.6416655$,

$-.0 \varepsilon 01999,0,0$;

$106,1,3,0.0,8.4016895,-.4587413,8.4016895,-.4587413,8.7666655$,

$-.4587413,0,0$;

$105,1,3,0.0,8.4016895, .3383414,8.4016895, .3383414,8.7666655$, $.3383414,0,0$;

$216,327,329,331,333,335,0,0$;

$212,1,5, .1226300, .0543000,1,1.5707963,0.0,0,0, .5245647$,

$-2.0293127,0.0,53.7971,0,0$;

$214,1, .1500000, .0500000,0.0, .6820347,-2.3987420, .6820347$,

$-2.0826126,0,0$;

$214,2, \ldots 500000, .0500000,0.0, .5820347,-1.6016597, .6820347$, $-1.9197176,0,0$;

$106,1,3,0.0, .1507847,-2.3987420, .1507847,-2.3987420, .8070347$,

$-2.3987420,0,0$

$106,1,3,0.0, .1507847,-1.6015597, .1507847,-1.6016597, .8070347$,

$-1.6015597,0,0$

$216,339,341,343,345,347,0,0$

$212,1,5, .1226300,00543000,1,1.5707963,0.0,0,0,3.2267876$,

$-1.98 \geq 3584,0.0,5 i .7971,0,0$;

$214,1, .1500000, .0500000,0.0,3.3842578,-2.3427420,3.3842578$,

$-2.6473156,0,0$;

$214,1, .1500000,00500000,0.0,3.3842578,-1.5456599,3.3842578$,

$-1.2160515,0,0$

$105, I, 3,0.0,2.8530078,-2.3 \div 27420,2.8530078,-2.3427420,3.509257 \varepsilon$,

$-2.3427420,0,0$;

$-v 0,1,3,0.0,2.3530078,-1.5456599,2.8530078,-1.5456599,3.5092578$,

$-1.5 \div 56599,0,0$

$216,351,353,355,357,359,0,0$;

$2: 2,1,5, .1226300, .0543000,1,1.5707963,0.0,0,0,5.7845654$,

- - .4505347,0.0,5i.7971,0,0;

$\therefore \therefore \ldots 505000, .0500000,0.0,5.6216655,-2.3347421,5.6216655$,

$-2 . ; 620077,0,0$;

$2: 4,2, .1500000, .0500000,0.0,5.6215655,-1.5376594,5.6216655$,

$-\therefore-1253847,5.7302631,-1.4233847,0,0$;

:こ5, :, 3, $0.0,5.0904155,-2.3347421,5.0904155,-2.3347421,5.7465655$,

$3132 \quad 289$

$3153 \quad 289$

$315 ? \quad 290$

$3178 \quad 291$

$317 P \quad 292$

319P 293

$319 \mathrm{P} \quad 294$

321? 295

$3212 \quad 296$

$323 P \quad 297$

$323 P \quad 298$

$325 P \quad 299$

$327 P \quad 300$

$327 P \quad 301$

$329 P \quad 302$

$329 P \quad 303$

331? 304

331P 305

$333 P \quad 306$

$333 P \quad 307$

$335 P \quad 308$

$335 \% \quad 309$

$337 P \quad 3 \geq 0$

$339 P \quad 311$

$339 P \quad 312$

$3412 \quad 313$

$341 P \quad 314$

$343 P \quad 315$

343 P 316

$345 P \quad 317$

$345 P \quad 3: 2$

$347 \mathrm{P} \quad 319$

$347 \mathrm{P} \quad 32 \mathrm{O}$

$349 ? \quad 321$

35ID 322

$351 P \quad 323$

$3538 \quad 324$

$353 P \quad 325$

$355 P \quad 326$.

उSEP $32 \bar{i}$

$3570 \quad 325$

$357 P \quad 325$

$359 \mathrm{P} \quad 33 \mathrm{C}$

$359 \mathrm{P}$ 35:

36IP 332

3639 33

$363 \mathrm{P} \quad 334$

365P 335

उESP 336

इ67? 337

$3675 \quad 338$

369? 32 
$-2.3347421,0,0$;

$106,1,3,0.0,5.0904155,-1.5376594,5.0904155,-1.5376594,5.7466655$,

$216,363,365,367,369,371,0,0$;

$212,1,5, .1226300, .0543000,1,1.5707963,0.0,0,0,8.6067367$,

$-1.3972015,0.0,54.7971,0,0$;

$214,3, .1500000, .0500000,0.0,8.4438868,-2.3107412,8.4438868$,

$-1.9121998,8.4438868,-1.3700516,8.5524864,-1.370051 .6,0,0$;

$214,1, .1500000, .0500000,0.0,8.4438868,-1.5136584,8.4438868$,

$-1.9121998,0,0$;

$106,1,3,0.0,7.9126368,-2.3107412,7.9126368,-2.3107412,8.5688868$,

$106,1,3,0.0,7.9126368,-1.5136584,7.9126368,-1.5136584,8.5688868$, $-1.5136584,0,0$;

$216,375,377,379,381,383,0,0$; 


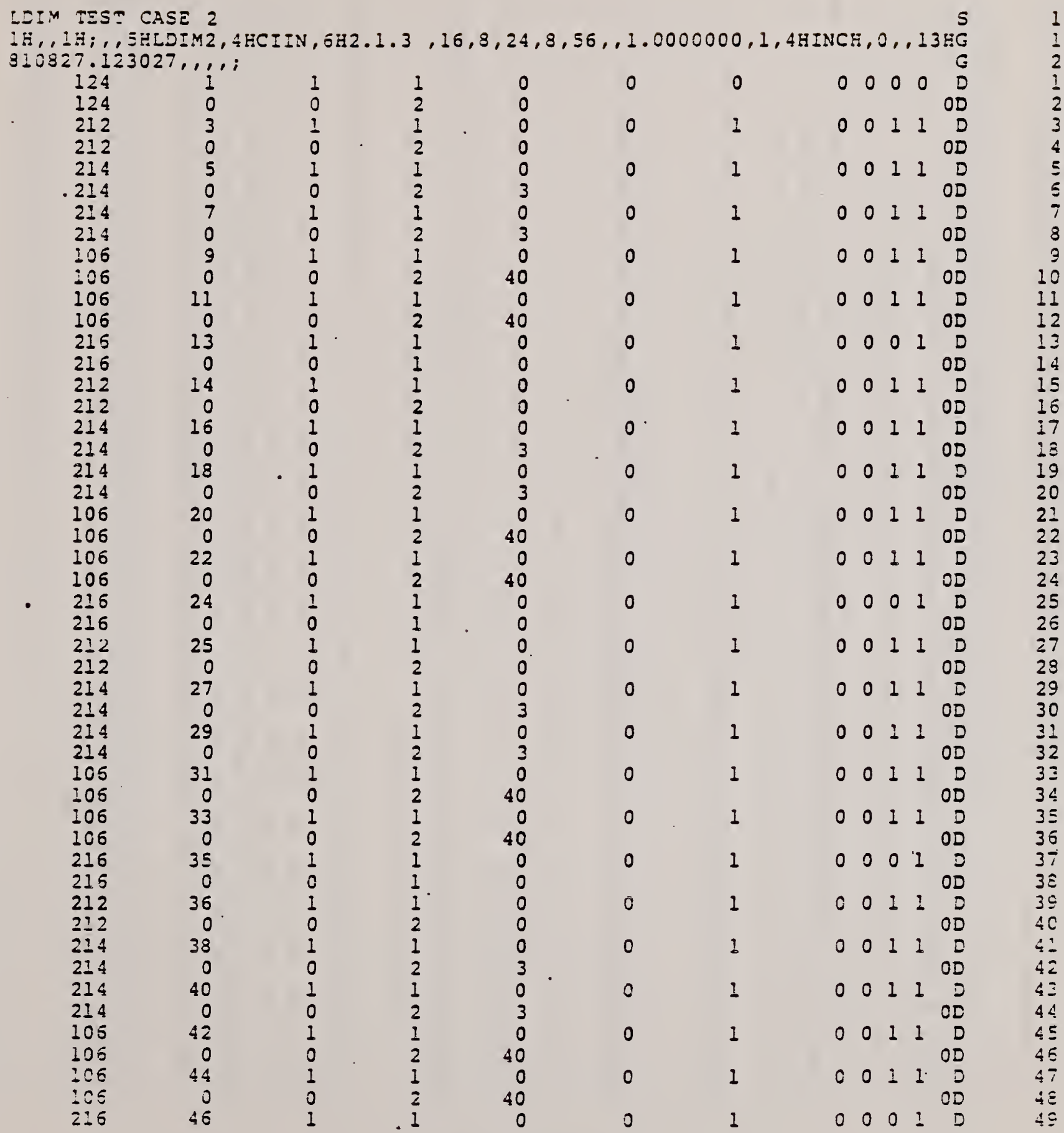




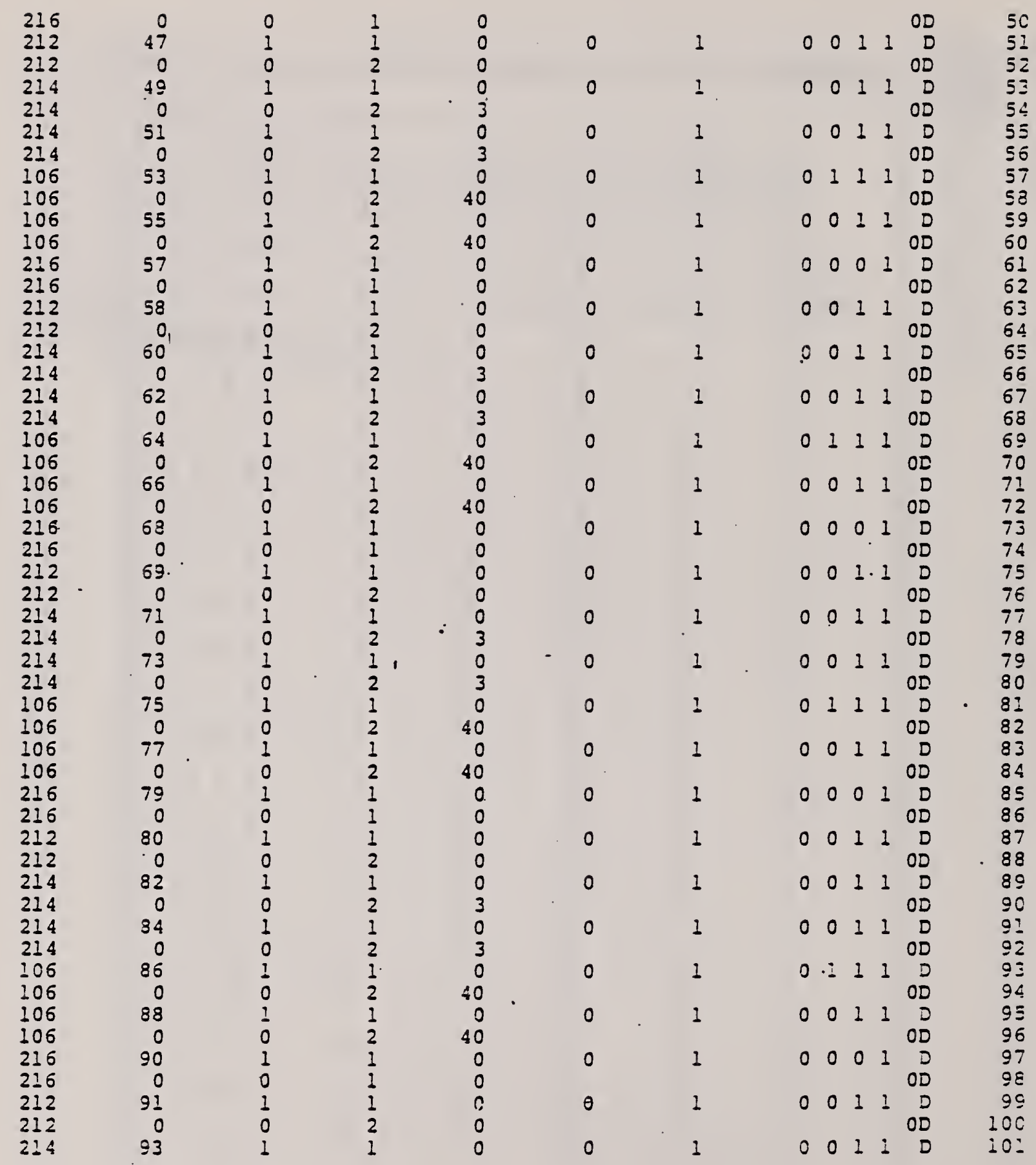




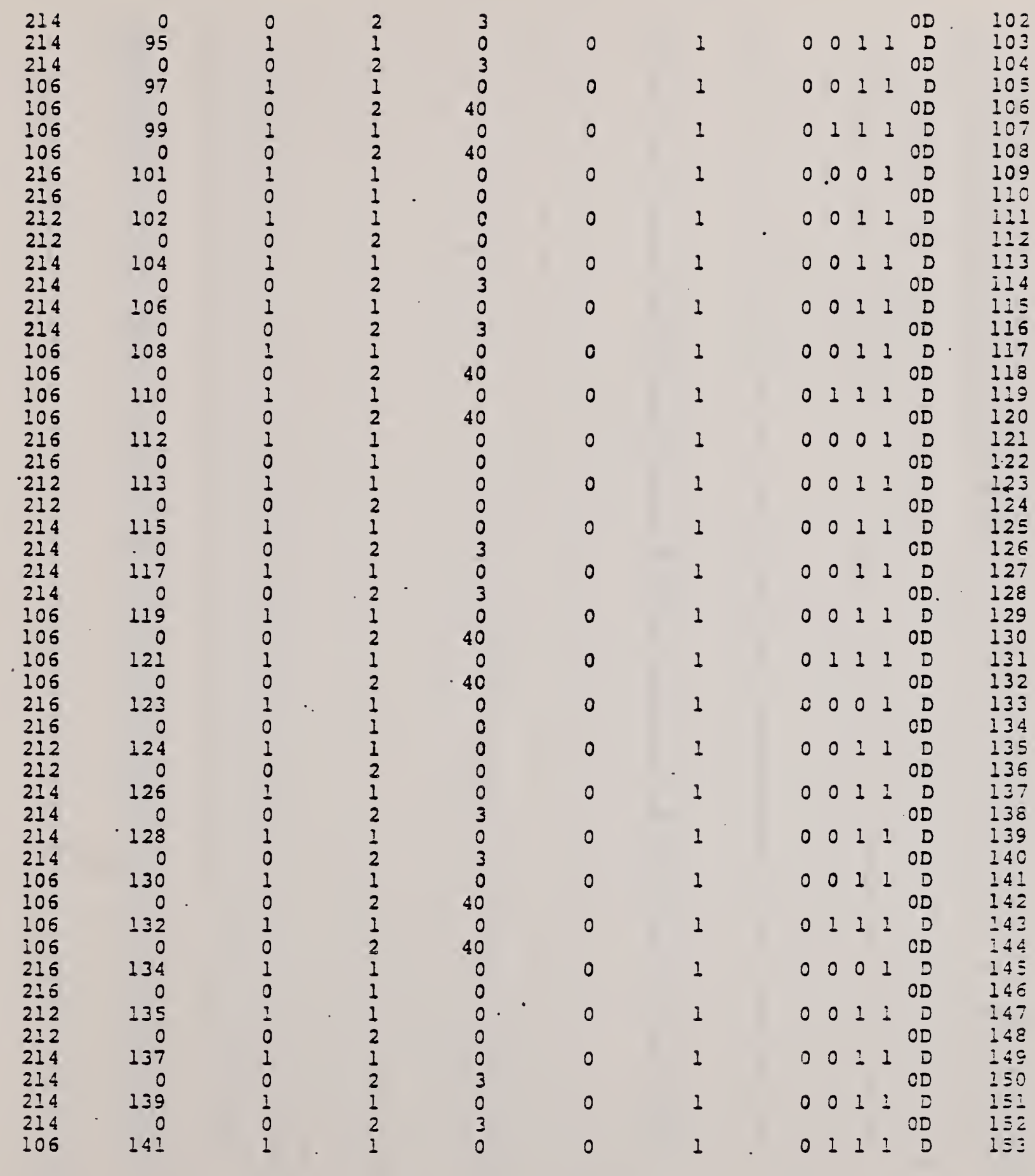




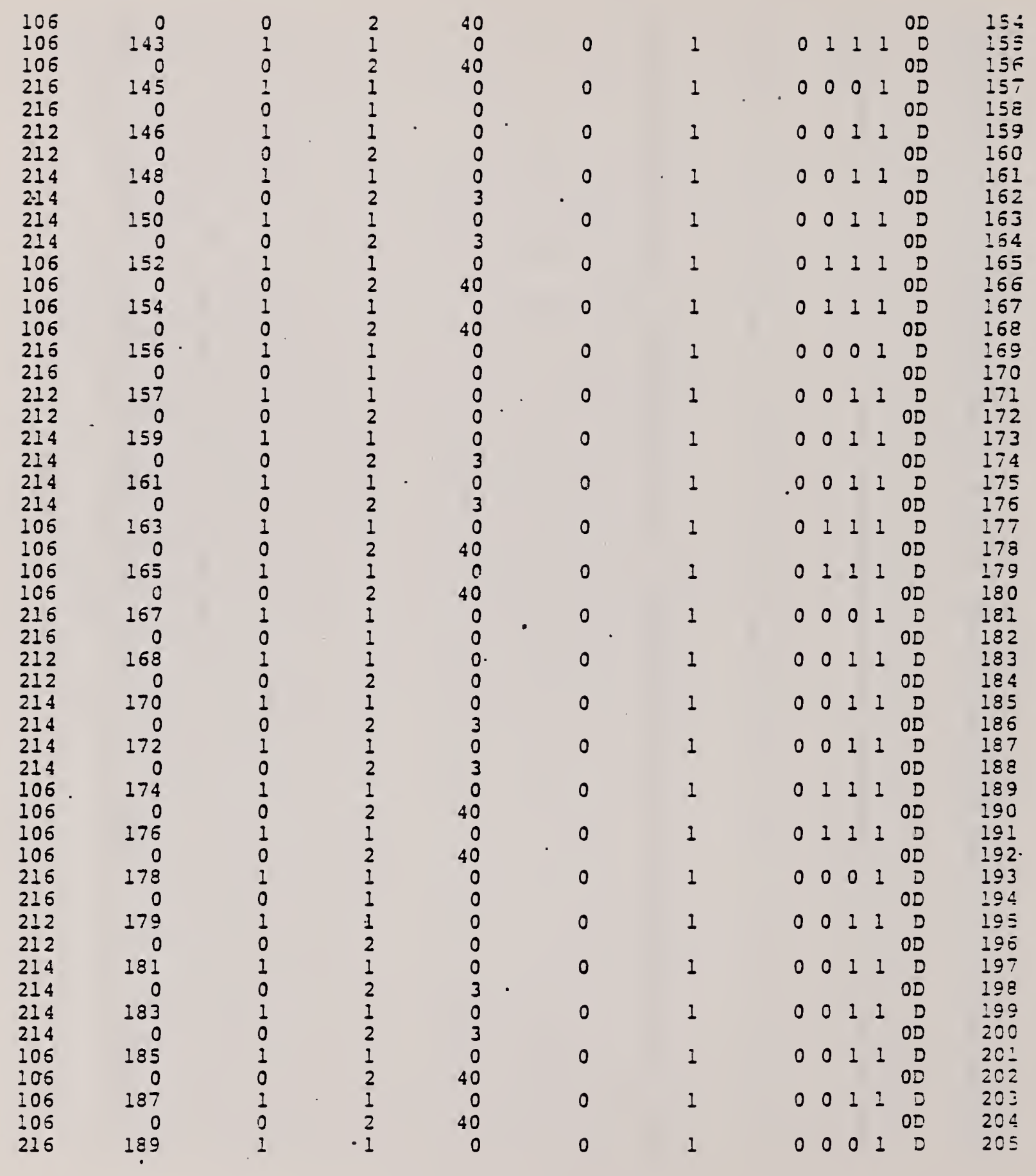




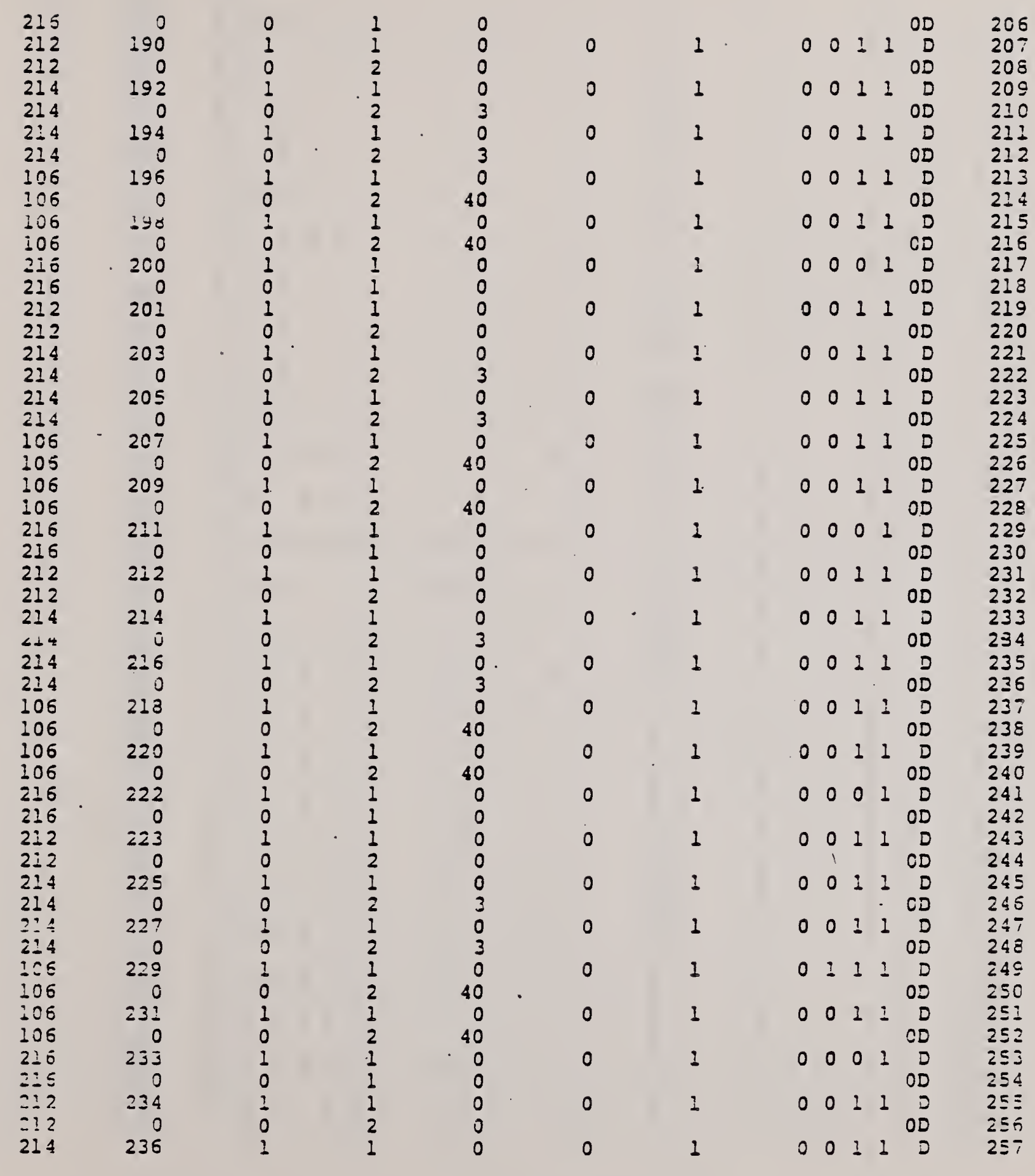




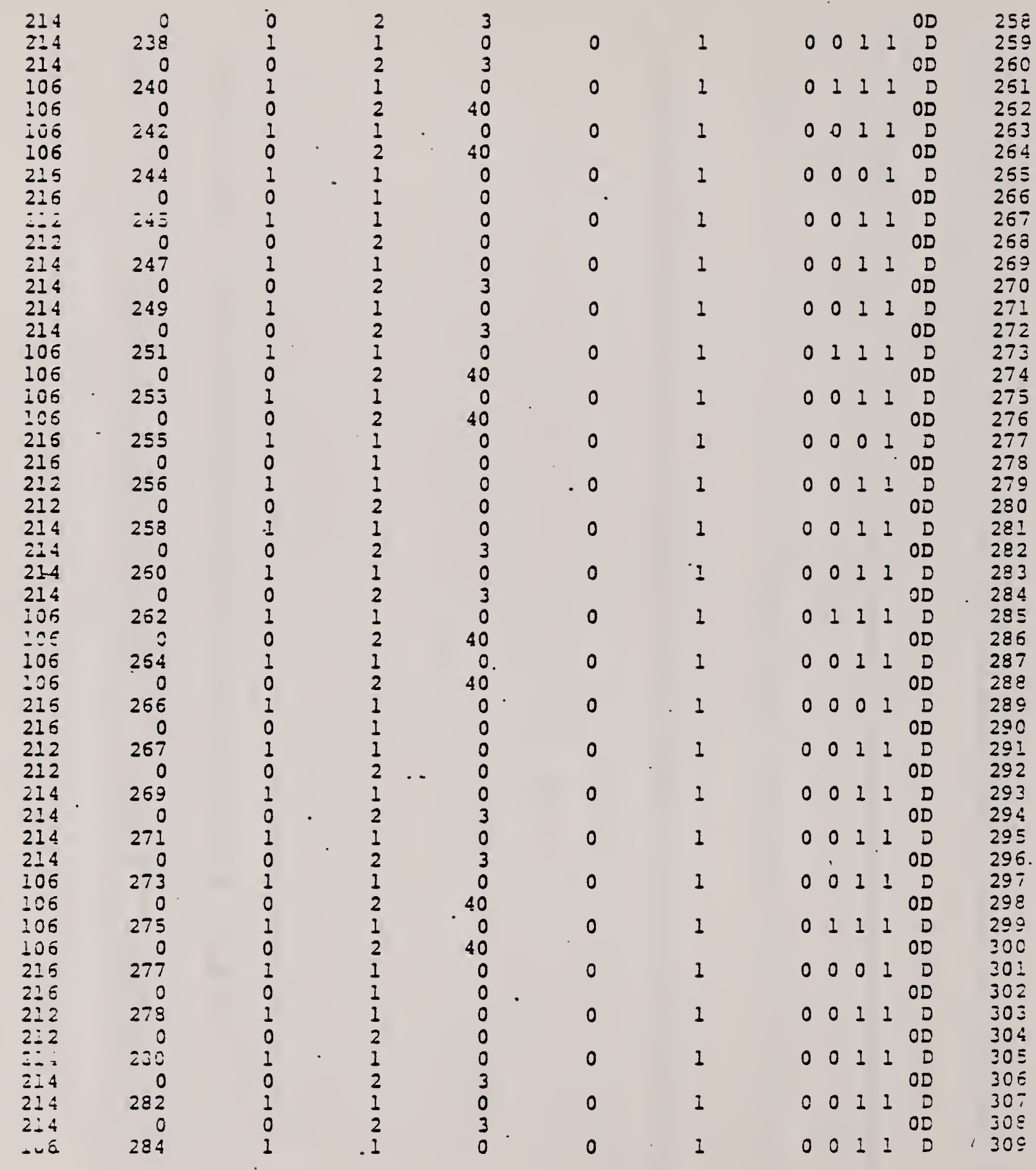




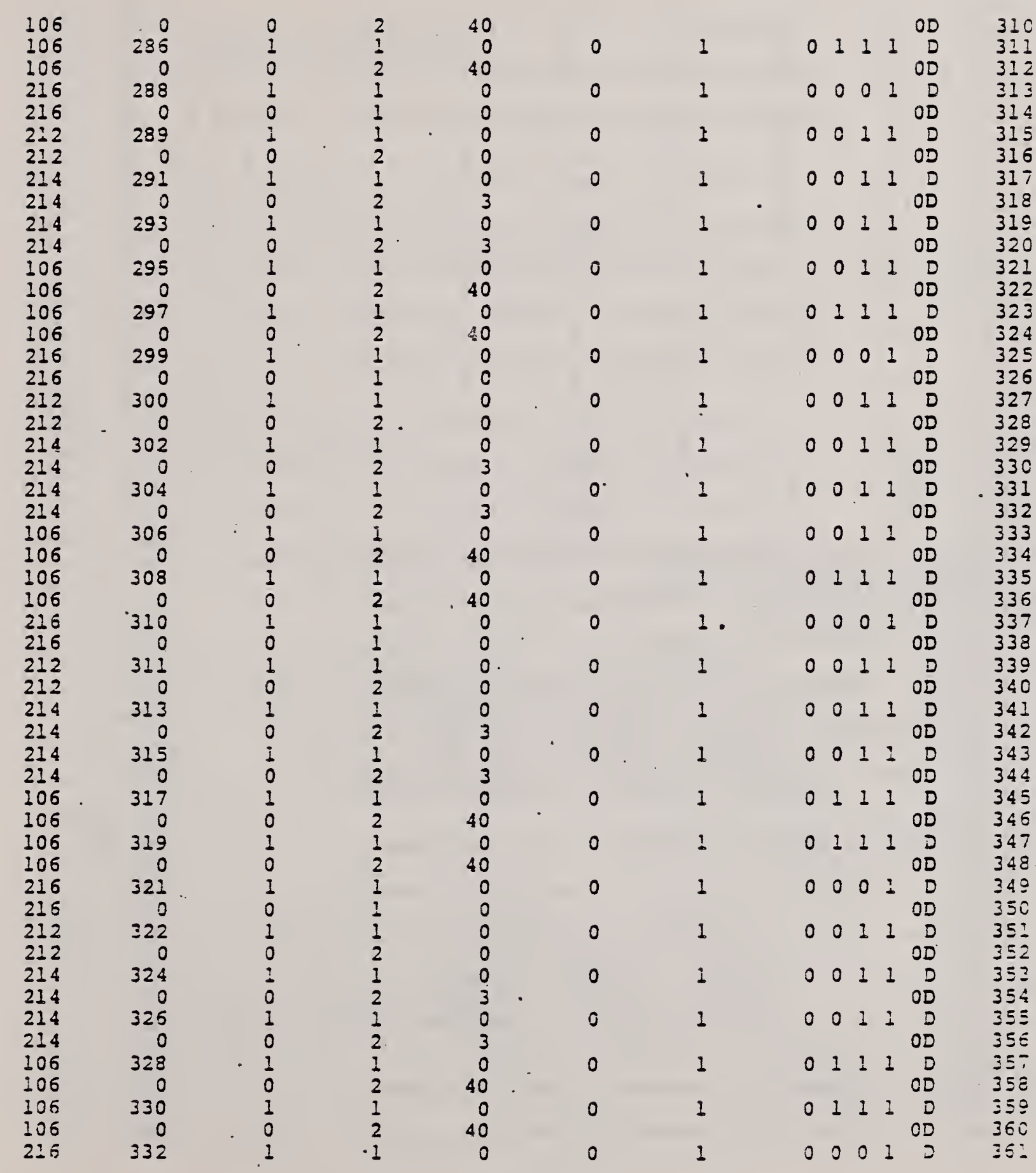




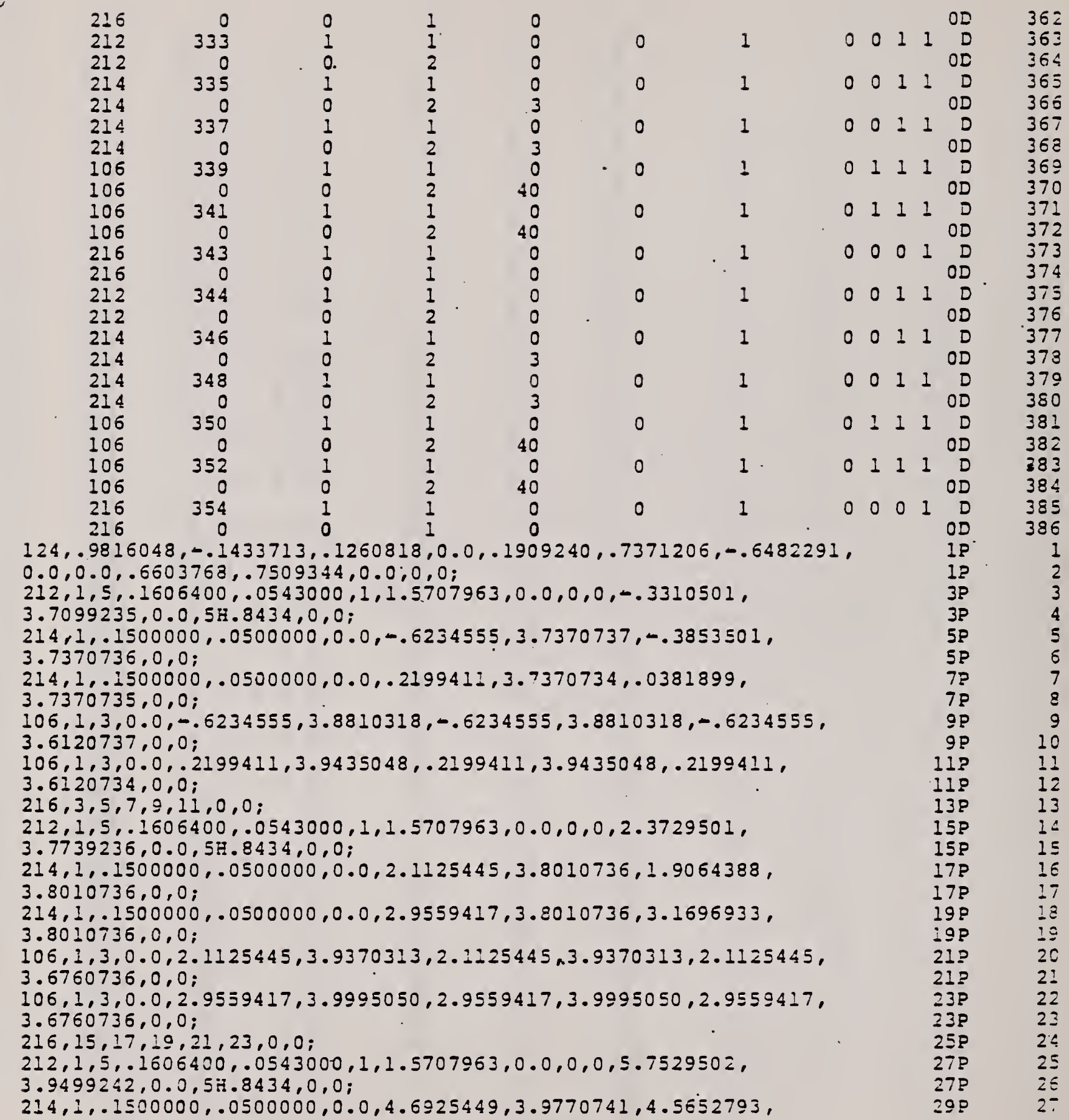


$3.9770741,0,0$;

$214,2, .1500000, .0500000,0.0,5.5359416,3.9770 .741,5.5900502$, $3.9770741,5.6986499,3.9770744,0,0$;

$106,1,3,0.0,4.6925449,3.9450312,4.6925449,3.9450312,4.5925449$, $3.852074,0,0,0$;

$106,1,3,0.0,5.5359416,4.0075045,5.5359416,4.0075045,5.5359416$, $3.8520741,0,0$;

$216,27,29,31,33,35,0,0$;

$212,1,5, .1606400, .0543000,1,1.5707963,0.0,0,0,8.5289497$,

$3.9659240,0.0,5 \pi .8434,0,0$;

$214,3, .1500000, .0500000,0.0,7.4125433,3.9930739,7.8342419$, $3.9930739,7.8342419,3.9930742,8.4746494,3.9930742,0,0$;

$214,1, .1500000, .0500000,0.0,8.2559404,3.9930739,7.8342419$, $3.9930739,0,0$;

$106,1,3,0.0,7.4125433,3.9690323,7.4125433,3.9690323,7.4125433$, $3.8680739,0,0$;

$106,1,3,0.0,8.2559404,4.0315061,8.2559404,4.0315061,8.2559404$, $3.8680739,0,0$;

$216,39,41,43,45,47,0,0$ :

$212,1,5, .1606400, .0543000,1,1.5707963,0.0,0,0,-.3790502$,

$1.6099244,0.0,54.8434,0,0$;

$214,1, .1500000, .0500000,0.0,-.6794549,1.6370744,-.4333502$ ，

$1.6370744,0,0$;

$214,1, .1500000, .0500000,0.0, .1639414,1.6370744,-.0098102$ ，

$1.6370744,0,0$;

$106,1,3,0.0,-.5794549,1.8035065,-.6794549,1.8035065,-.6794549$,

$1.5120744,0,0$;

$106,1,3,0.0, .1639414,1.8035065, .1639414,1.8035065, .1639414$,

$1.5120744,0,0$;

$216,51,53,55,57,59,0,0$;

$212,1,5, .1606400, .0543000,1,1.5707963,0.0,0,0,2.3489499$,

$1.6419244,0.0,5 \mathrm{H} .8434,0,0$ :

$214,1, .1500000, .0500000,0.0,2.0565453,1.6690743,1.8184404$,

1.6690743,0,0;

$214,1, .1500000, .0500000,0.0,2.8999419,1.6690743,3.0816940$,

$1.6690743,0,0$;

$106,1,3,0.0,2.0565453,1.8595064,2.0565453,1.8595064,2.0565453$, i. $5440743,0,0$;

$106,1,3,0.0,2.8999419,1.8595064,2.8999419,1.8595064,2.8999419$,

$1.5440743,0,0$;

$216,63,65,67,69,7 \pm, 0,0$ :

$212,1,5, .1506400, .0543000,1,1.5707963,0.0,0,0,5.8249497$,

$1.7299243,0.0,54.8434,0,0$;

$214,1, .1500000, .0500000,0.0,4.5365452,1.7570742,4.5092796$,

$1.7570742,0,0$;

$214,2, .1500000,00500000,0.0,5.4799418,1.7570742,5.5020498$,

$1.7570742,5.7706494,1.7570742,0,0$;

$106,1,3,0.0,4.6365452,1.8675066,4.6365452,1.8675066,4.6365452$,

$1.6320742,0,0$;

$106,1,3,0.0,5.4799418,1.3675060,5.4799418,1.8675066,5.4799418$,

$1.6320742,0,0$;

$216,75,77,79,81,83,0,0$ :

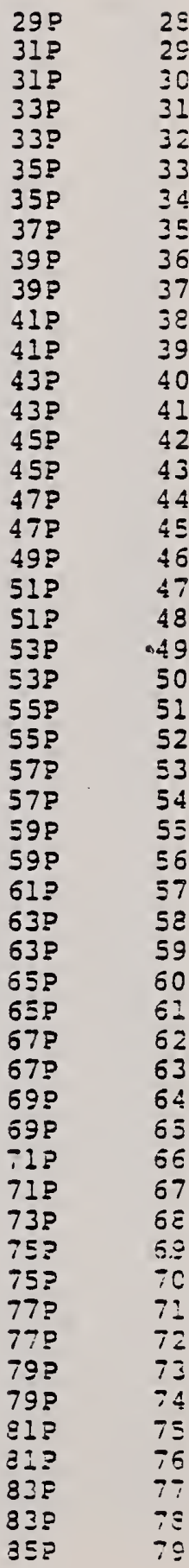


$212,1,5, .1606400, .0543000,1,1.5707963,0.0,0,0,8.5049496$, ..7699242,0.0,5ï.8434,0,0;

$2 \vdots 4,3, .1500000, .0500000,0.0,7.3565435,1.7970741,7.7782421$, $1.7970741,7.7782421,1.7970741,8.4506493,1.7970741,0,0$;

$214,1, .1500000, .0500000,0.0,8.1999407,1.7970741,7.7782421$, $1.7970741,0,0$ :

$106,1,3,0.0,7.3565435,1.8915074,7.3565435,1.8915074,7.3565435$, $1.6720741,0,0$;

$105,1,3,0.0,8.1999407,1.8915074,8.1999407,1.8915074,8.1999407$, $1.572074: 0,0$ :

$216,87,89,91,93,95,0,0$;

$212,1,5, .1606400, .0543000,1,1.5707963,0.0,0,0,-.2470502$,

$-.5950753,0.0,53.8434,0,0$;

$214,1, .1500000, .0500000,0.0,-.5714565,-.8709253,-.3013502$,

$-.8709253,0,0$

$214,1, .1500000, .0500000,0.0 \%, .2719398,-.8709253, .1221898$, $-.8709253,0,0$

$106,1,3,0.0,-.5714565,-.7029657,-.5714565,-.7029657,-.5714565$,

$-.9959253,0,0$;

$106,1,3,0.0, .2719398,-.7029657, .2719398,-.7029657, .2719398$,

$-.9959253,0,0$;

$216,99,101,103,105,107,0,0$;

$212,1,5, .1606400, .0543000,1,1.5707963,0.0,0,0,2.5129499$,

$-.8500753,0.0,57.8434,0,0 ;$

$2 i 4, \therefore, .1500000, .0500000,0.0,2.1645436,-.8229253,1.8704371$,

$-.8229253,0,0$;

$214,1, .1500000, .0500000,0.0 ., 3.0079403,-.8229253,3.1336907$,

$-.8229253,0,0$;

$106,1,3,0.0,2.1645436,-.6469661,2.1645436,-.6469661,2.1645436$,

$-. y 4 ., y<j j, 0,0$;

$106,1,3,0.0,3.0079403,-.6469661,3.0079403,-.6469561,3.0079403$,

$-.9479253,0,0$;

$216,111,113,115,117,119,0,0$;

$212,1,5, .1606400, .0543000,1,1.5707963,0.0,0,0,5.7969503$,

$-.7860753,0.0,5 \mathrm{H} .8434,0,0$;

$214,1, .1500000, .0500000,0.0,4.7445436,-.7589253,4.6172780$,

$-.7589253,0,0$;

$214,2, .1500000, .0500000,0.0,5.5879402,-.7589254,5.6340504$,

$-.7589254,5.7426500,-.7589253,0,0$;

$106,1,3,0.0,4.7445436,-.6389657,4.7445436,-.6389657,4.7445436$,

$-.8839253,0,0$;

$106,1,3,0.0,5.5879402,-.6389657,5.5879402,-.6389657,5.5879402$, $-.2 ?: 0254,0.0$;

$216,123,125,127,129,131,0,0$;

$2=2, i, 5, .1506400, .0543000,1,1.5707963,0.0,0,0,8.5329494$,

$-.5740752,0.0,5 \mathrm{H} .8434,0,0$;

$2: 4,1, .1500000, .0500000,0.0,7.4645410,-.6469253,7.8862398$,

$-.6459253,0,0$ :

$214,3, .1500000,00500000,0.0,8.3079386,-.6469253,7.8862398$,

-. $\{552253,7.8852398,-.6469253,8.4786491,-.6469253,0,0$;

:. . $, 1,3,0.0,7.4645410,-.6149648,7.4645410,-.6149648,7.4645410$,

$-.7719253,0,0$;

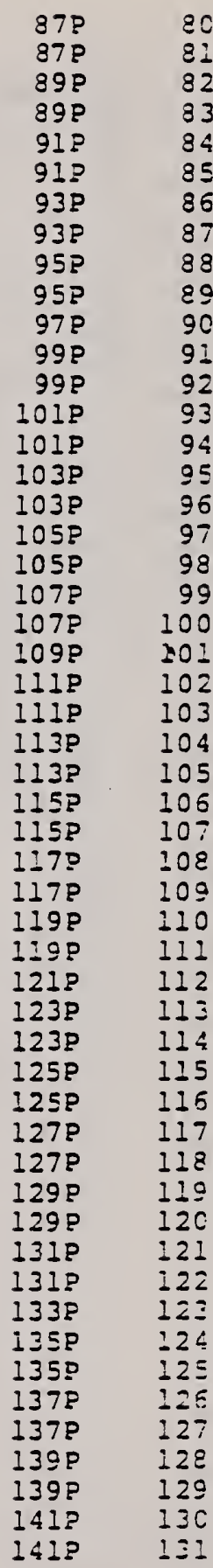


$106,1,3,0.0,8.3079386,-.6149648,8.3079386,-.5149648,8.3079386$, $-.7719253,0,0$

$216,135,137,139,141,143,0,0$;

$212,1,5, .1606400, .0543000,1,1.5707963,0.0,0,0,-.3630502$,

$-2.5500751,0.0,5 \mathrm{H} .8434,0,0$;

$214,3, .1500000, .0500000,0.0,-.6474565,-2.5229250,-.2257583$,

$-2.5229250,-.2257533,-2.5229251,-.4173502,-2.5229251,0,0$;

$214,1, .1500000, .0500000,0.0, .1959399,-2.5229250,-.2257583$,

$-2.5229250,0,0$;

$106,1,3,0.0,-.6474565,-1.9916750,-.6474565,-1.9916750,-.6474565$,

$-2.6479250,0,0$;

$106,1,3,0.0, .1959399,-1.9916750, .1959399,-1.9916750, .1959399$,

$-2.6479250,0,0$;

$216,147,149,151,153,155,0,0$;

$212,1,5, .1606400, .0543000,1,1.5707963,0.0,0,0,2.3489499$,

$-2.5020752,0.0,5 \% .8434,0,0$;

$214,1, .1500000, .0500000,0.0,2.0885434,-2.4749253,1.8824368$,

$-2.4749253,0,0$;

$214,1, .1500000, .0500000,0.0,2.9319401,-2.4749253,3.1456903$,

$-2.4749253,0,0$;

$106,1,3,0.0,2.0885434,-1.9436753,2.0885434,-1.9436753,2.0885434$,

$-2.5999253,0,0$ :

$106,1,3,0.0,2.9319401,-1.9436753,2.9319401,-1.9436753,2.9319401$,

$-2.5999253,0,0$;

$216,159,161,163,165,167,0,0$;

$212,1,5, .1606400, .0543000,1,1.5707963,0.0,0,0,5.6409497$,

$-2.4700751,0.0,5 H .8434,0,0$;

$214,1, .1500000, .0500000,0.0,4.6585438,-2.4429251,4.5412782$ ，

$-2.4429251,0,0$;

$214,2, .1500000, .0500000,0.0,5.5119405,-2.4429251,5.5458312$,

$-2.4429251,5.5866494,-2.4429252,0,0$;

$106,1,3,0.0,4.6685438,-1.9116751,4.6685438,-1.9116751,4.6685438$, $-2.5679251,0,0$

$106,1,3,0.0,5.5119405,-1.9116751,5.5119405,-1.9116751,5.5119405$,

$-2.5679251,0,0$;

$216,171,173,175,177,179,0,0$;

$212,1,5, .1606400, .0543000,1,1.5707963,0.0,0,0,8.4459953$,

$-2.4373329,0.0,5 \mathrm{H} .8434,0,0$;

$214,3, .5500000, .0500000,0.0,7.3885422,-2.4101831,7.8102407$,

$-2.4101831,7.8102407,-2.4101830,8.3916950,-2.4101830,0,0$;

$214,1, .1500000, .0500000,0.0,8.2319393,-2.4101831,7.8102407$,

$-2.4101831,0,0$;

$106,1,3,0.0,7.3885422,-1.8789331,7.3885422,-1.8789331,7.3885422$, $-2.5351831,0,0$;

$106,1,3,0.0,8.2319393,-1.8789331,8.2319393 \cdot,-1.8789331,8.2319393$,

$-2.5351831,0,0$;

$216,183,185,187,189,191,0,0$;

$212,1,5, .1226300, .0543000,1,1.5707963,0.0,0,0, .5227866$,

$4.4041305,0.0,51.7971,0,0$;

$214,1, .1500000, .0500000,0.0, .6802565,4.0372548, .6802565$,

$4.3498306,0,0$;

$214,1, .1500000, .0500000,0.0, .6802565,4.8343368, .680255 \equiv$,

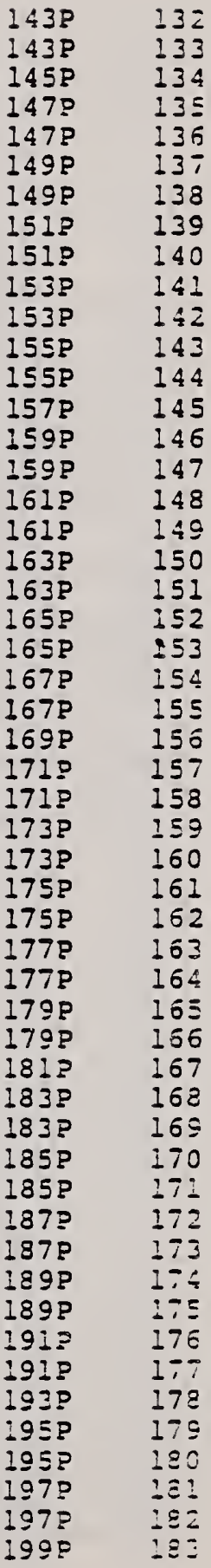


$4.5127311,0,0$;

$106,1,3,0.0, .3136918,4.0372548, .3136918,4.0372548, .8052565$, $4.0372548,0,0$;

$106,1,3,0.0, .2759593,4.8343368, .2759593,4.8343368, .8052565$, $4.8343358,0,0$;

$216,195,197,199,201,203,0,0$

$212,1,5, .1226300, .0543000,1,1.5707963,0.0,0,0,3.2907867$,

$4.4521308,0.0,5 \mathrm{H} .7971,0,0$;

$214,1, .1500000, .0500000,0.0,3.4482570,4.0932550,3.4482570$,

$3.7886791,0,0$ :

$714.1 .1500000, .0500000,0.0,3.4482570,4.8903365,3.4482570$,

$5.2199416,0,0$;

$106,1,3,0.0,3.0496922,4.0932550,3.0496922,4.0932550,3.5732570$,

$4.0932550,0,0$;

$106,1,3,0.0,3.0119591,4.8903365,3.0119591,4.8903365,3.5732570$, $4.8903365,0,0$;

$216,207,209,211,213,215,0,0$;

$212,1,5, .1226300, .0543000,1,1.5707963,0.0,0,0,5.8556762$,

$5.0316853,0.0,5 \mathrm{H} .7971,0,0$;

$214,1, .1500000, .0500000,0.0,5.6927762,4.1012545,5.6927762$,

$3.9739889,0,0$

$214,2, .1500000, .0500000,0.0,5.6927752,4.8983369,5.6927762$,

$5.0588365,5.8013759,5.0588365,0,0$;

$106,1,3,0.0,5.6296926,4.1012545,5.6296926,4.1012545,5.8177762$, $4.1012545,0,0$;

$106,1,3,0.0,5.5919585,4.8983369,5.5919585,4.8983369,5.8177762$, $4.8983369,0,0$;

$216,219,221,223,225,227,0,0$;

$212,1,5, .1226300, .0543000,1,1.5707963,0.0,0,0,8.6423426$,

E. : $2 \div ? \geq ?: 0.0,5 \mathrm{H} .7971,0,0$;

$214,3, .1500000, .0500000,0.0,8.4794426,4.1252561,8.4794426$,

4. $5237970,8.4794426,5.0499473,8.5880423,5.0499473,0,0$;

$214,1, .1500000, .0500000,0.0,8.4794426,4.9223380,8.4794426$, $4.5237970,0,0$;

$106,1,3,0.0,8.3496914,4.1252561,8.3496914,4.1252561,8.6044426$, $4.1252561,0,0$;

$106,1,3,0.0,8.3119574,4.9223380,8.3119574,4.9223380,8.6044426$, $4.9223380,0,0$;

$216,231,233,235,237,239,0,0$;

$212,1,5, .1226300,00543000,1,1.5707963,0.0,0,0, .5067868$,

$2.2739091,0.0,5 \mathrm{H} .7971,0,0$;

$214,1, .1500000, .0500000,0.0, .6642568,1.8972565, .6642568$,

2.2:06094,0,0;

$214,1, .1500000,00500000,0.0,06642568,2.5943388, .6642568$,

$2.3825092,0,0$;

$106,1,3,0.0, .2199603,1.8972565, .2199603,1.8972565, .7892568$,

i. $8972565,0,0$;

$106,1,3,0.0, .2199603,2.6943388, .2199603,2.6943388, .7892568$,

$2.6943388,0,0$ :

$2 \div 0,243,245,247,249,251,0,0$;

$222,1, \equiv, .1226300, .0543000,1,1.5707963,0.0,0,0,3.1734543$,

$2.233354: 0.0,5$ H. $7971,0,0$; 
Z: $1,1, .1500000, .0500000,0.0,3.3309245,1.9532561,3.3309245$,

$1.5424580,0,0$ :

$214,1, .1500000, .0500000,0.0,3.3309245,2.7503386,3.3309245$, $3.0737228,0,0$;

$106,1,3,0.0,2.9559598,1.9532561,2.9559598,1.9532561,3.4559245$, $1.0532561,0,0$;

$106,1,3,0.0,2.9559598,2.7503386,2.9559598,2.7503386,3.4559245$, $2.7503385,0,0$;

$210,255,25 i, 259,261,263,0,0$;

ᄀi=..5..1726300,.0543000,1,1.5707963,0.0,0,0,5.7490096, $2.9850202,0.0, \equiv H .7971,0,0$;

$214,1, .1500000, .0500000,0.0,5.5861096,1.9612564,5.5861 .096$,

$1.8339908,0,0$;

$214,2, .1500000, .0500000,0.0,5.5861096,2.7583389,5.5861096$,

$3.0121703,5.6947093,3.0121703,0,0$;

$100,1,3,0.0,5.5359597,1.9612564,5.5359597,1.9612564,5.7111096$, $1.9612564,0,0$;

$106,1,3,0.0,5.5359597,2.7583389,5.5359597,2.7533389,5.7111096$, $2.7583382,0.0$

$216,267,269,271,273,275,0,0$;

$212,1,5, .1226300, .0543000,1,1.5707963,0.0,0,0,8.5890093$,

$2.9316869,0.0,53.7971,0,0$;

$224,3, .1500000, .0500000,0.0,8.4261093,1.9852574,8.4261093$,

$2.3837985,8.4261093,2.9588370,8.5347090,2.9588370,0,0$;

$214,1, .1500000, .0500000,0.0,8.4261093,2.7823396,8.4261093$, $2.3837985,0,0$;

$106,1,3,0.0,8.2559586,1.9852574,8.2559586,1.9852574,8.5511093$, $1.0852574,0,0$;

$106,1,3,0.0,8.2559586,2.7823396 ; 3.2559586,2.7823396,8.5511093$,

$2.7823396,0,0$;

$216,270.281,283,285,287,0,0$;

$212, I, 5, .1226300, .0543000,1,9.5707963,0.0,0,0, .6334534$,

$-.12 \$ 3128,0.0,5 \% .7971,0,0$;

$214,1, .1500000, .0500000,0.0, .7909234,-.5467421, .7909234$,

$-.1825127,0,0$ :

$214,1, .1500000, .0500000,0.0, .7909234, .2503400, .7909234$,

$-.0197127,0,0$;

$105,1,3,0.0, .3656907,-.5467421, .3656907,0.5467421, .9159234$,

$-.5467421,0,0$;

$106,1,3,0.0, .3656907, .2503400, .3656907, .2503400, .9159234$,

$.2503400,0,0$;

$216,291,293,295,297,299,0,0$;

: $=, \quad, \quad, .2225300, .0543000,1,1.5707963,0.0,0,0,3.3890090$ ，

$-.1283129,0.0,5 \mathrm{H} .7971,0,0$;

$214,1, .1500000, .0500000,0.0 .3 .5464792,-.4907423,3.5464792$,

$-.798971 ?, 0,0$ :

$214,1, .1500000, .0500000,0.0,3.5464792, .3063399,3.5464792$,

. $57 \div 30=7,0,0 ;$

ICE, 1,3,0.0,3.1015908,-.4907423,3.1016908,-.4907423,3.6714792,

$-.4507423,0,0$;

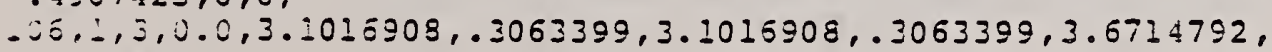

$.2053399,0,0$;

$\begin{array}{ll}257 P & 236 \\ 257 P & 237 \\ 259 P & 238 \\ 259 P & 239 \\ 261 P & 240 \\ 261 P & 241 \\ 253 P & 242 \\ 263 P & 243 \\ 265 P & 244 \\ 267 P & 245 \\ 267 P & 246 \\ 269 P & 247 \\ 269 P & 248 \\ 271 P & 249 \\ 271 P & 250 \\ 273 P & 251 \\ 273 P & 252 \\ 275 P & 253 \\ 275 P & 254 \\ 277 P & 255 \\ 279 P & 256 \\ 279 P & 257 \\ 281 P & 258 \\ 281 P & 259 \\ 283 P & 260 \\ 283 P & 261 \\ 285 P & 262 \\ 295 P & 263 \\ 287 P & 264 \\ 287 P & 255 \\ 289 P & 256 \\ 291 P & 267 \\ 291 P & 268 \\ 293 P & 269 \\ 293 P & 270 \\ 295 P & 271 \\ 295 P & 272 \\ 297 P & 273 \\ 297 P & 274 \\ 299 P & 275 \\ 299 P & 27 \Xi \\ 301 P & 277 \\ 303 P & 278 \\ 303 P & 279 \\ 305 P & 280 \\ 305 P & 28 I \\ 307 P & 282 \\ 307 P & 283 \\ 309 P & 284 \\ 309 P & 28 \Xi \\ 311 P & 28 E \\ 311 P & 28 T \\ & \end{array}$


$216,303,305,307,309,311,0,0$;

$212,1,5, .1226300, .0543000,1,1.5707963,0.0,0,0,5.8578987$, $.5027984,0.0,5 \mathrm{H} .7971,0,0$;

$214,1, .1500000, .0500000,0.0,5.5949987,-.4827421,5.6949987$,

$-.6100077,0,0$;

$214,2, .1500000, .0500000,0.0,5.6949987, .3143407,5.6949987$,

$.5299483,5.8035984, .5299483,0,0$;

$106,1,3,0.0,5.6816912,-.4827421,5.6816912,-.4827421,5.8199987$,

$-.4827421,0,0$ :

$106,1,3,0.0,5.6816912, .3143407,5.6816912, .3143407,5.8199987$,

$.3143407,0,0$;

$216,315,317,319,321,323,0,0$;

$212,1,5, .1226300, .0543000,1,1.5707963,0.0,0,0,8.8045654$,

$.4672429,0.0,5 \mathrm{H} .7971,0,0$;

$214,3, .1500000, .0500000,0.0,8.6416655,-.4587413,8.6416655$,

$-.0601999,8.6416655, .4943928,8.7502651, .4943928,0,0$;

$214,1, .1500000, .0500000,0.0,8.6416655, .3383414,8.6416655$, $-.0601999,0,0$;

$106,1,3,0.0,8.4016895,-.4587413,8.4016895,-.4587413,8.7666655$,

$-.4587413,0,0$;

$106,1,3,0.0,8.4016895, .3383414,3.4016895, .3383414,8.7666655$,

$.3383414,0,0$;

$216,327,329,331,333,335,0,0$;

$212,1,5, .1226300, .0543000,1,1.5707963,0.0,0,0, .5245647$,

$-2.0283127,0.0,5 \mathrm{H} .7971,0,0$ :

$214,1, .1500000, .0500000,0.0, .6820347,-2.3987420, .6820347$,

$-2.0826126,0,0$;

$214,1, .1500,000, .0500000,0.0, .6820347,-1.6016597, .6820347$,

$-1.9197126,0,0$;

$106,1,3,0.0, .1507847,-2.3987420, .1507847,-2.3987420, .8070347$,

$-2.3987420,0,0$ :

$106,1,3,0.0, .1507847,-1.6016597, .1507847,-1.6016597, .8070347$,

$-1.6016597,0,0$;

$216,339,341,343,345,347,0,0$;

$212,1,5, .1226300, .0543000,1,1.5707963,0.0,0,0,3.2267876$,

$-1.9838684,0.0,5 \% .7971,0,0$;

$214,1, .1500000, .0500000,0.0,3.3842578,-2.3427420,3.3842578$,

$-2.6473156,0,0$;

$214,1, .1500000, .0500000,0.0,3.3842578,-1.5456599,3.3842578$,

$-1.2160515,0,0$;

$106,1,3,0.0,2.8530078,-2.3427420,2.3530078,-2.3427420,3.5092578$, $-2.3427420,0,0$;

$106,1,3,0.0,2.8530078,-1.5456599,2.8530078,-1.5456599,3.5092578$,

$-1.5456599^{\prime \prime}, 0,0$;

$215,351,353,355,357,359,0,0$;

$212,1,5, .1226300, .0543000,1,1.5707963,0.0,0,0,5.7845054$,

$-1.4505347,0.0,5 \mathrm{H} .7971,0,0$;

$214,1, .1500000, .0500000,0.0,5.5216655,-2.3347421,5.6216655$,

$-2.4620077,0,0$;

$214,2, .1500000, .0500000,0.0,5.6216655,-1.5376594,5.6216655$,

$-1.4233847,5.7302551,-1.4233847,0,0$ :

$106,1,3,0.0,5.0904155,-2.3347421,5.0904155,-2.3347421,5.7466655$, 


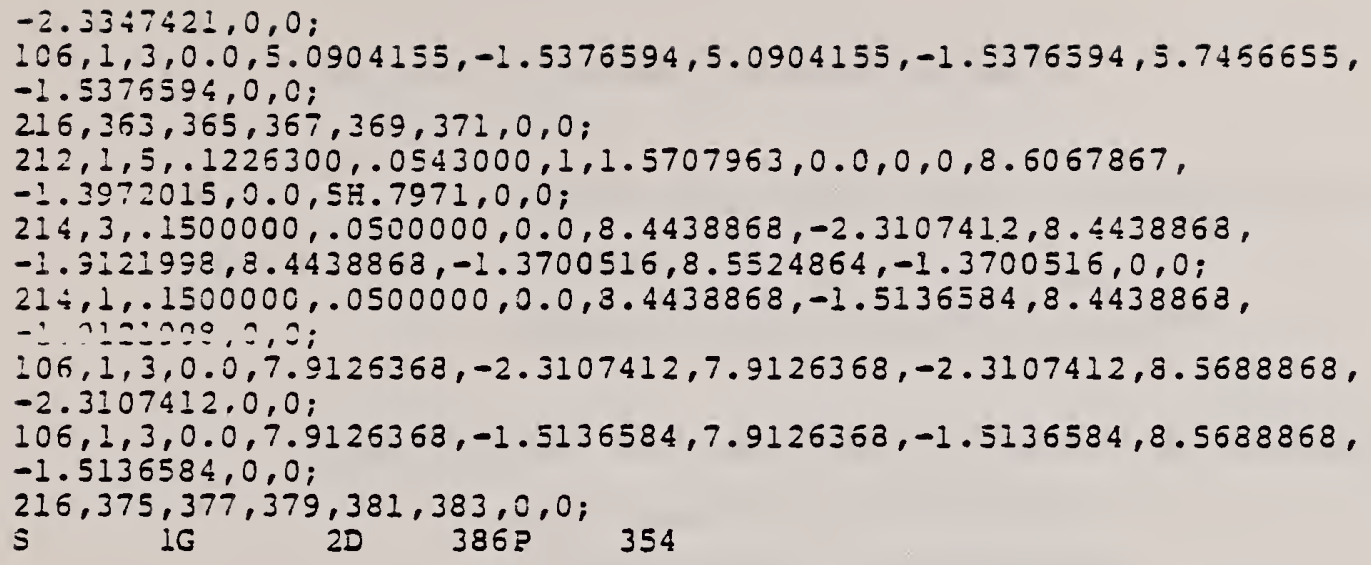





\subsubsection{Radius Dimension}

The radius dimensions contain subentities which include leaders, arc center points and general notes.

The following conventions were followed:

- general note sub-entities to be single text string with no box rotation

- leaders to have two or three segments

Case 1 (Figures 3.3.14-1 and 3.3.14-2) tests the following:

- text in, arrows in configuration

- text out, arrows in configuration

- variable length horizontal leader segments

- leader originating from head of text

- leader originating from sail of text

Case 2

- This is identical to Case 1 . except non-model space with rotation $\left(-60.34^{\circ}, 3.63^{\circ}, 2.06^{\circ}\right)$ is used per section 3.0 

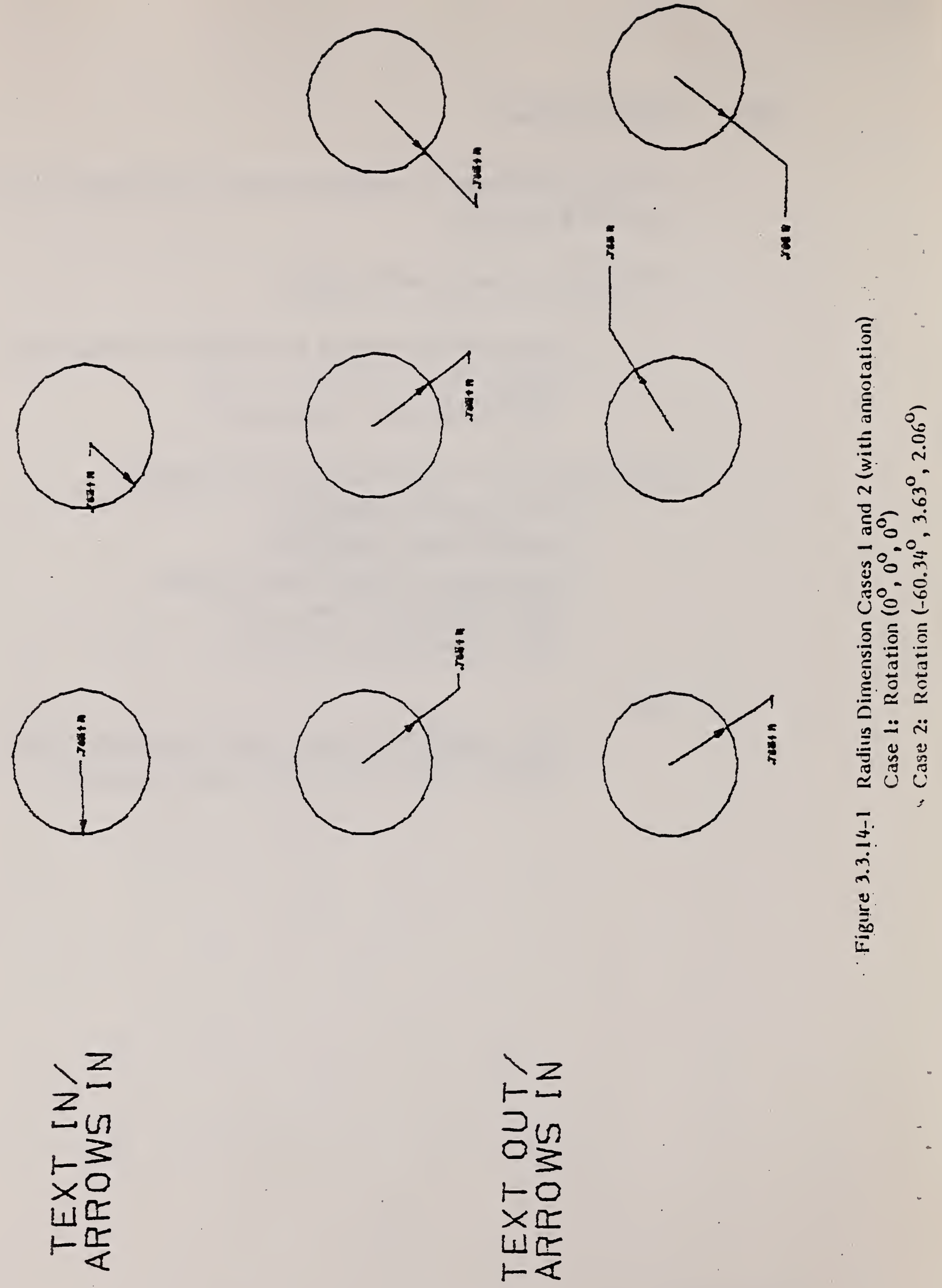

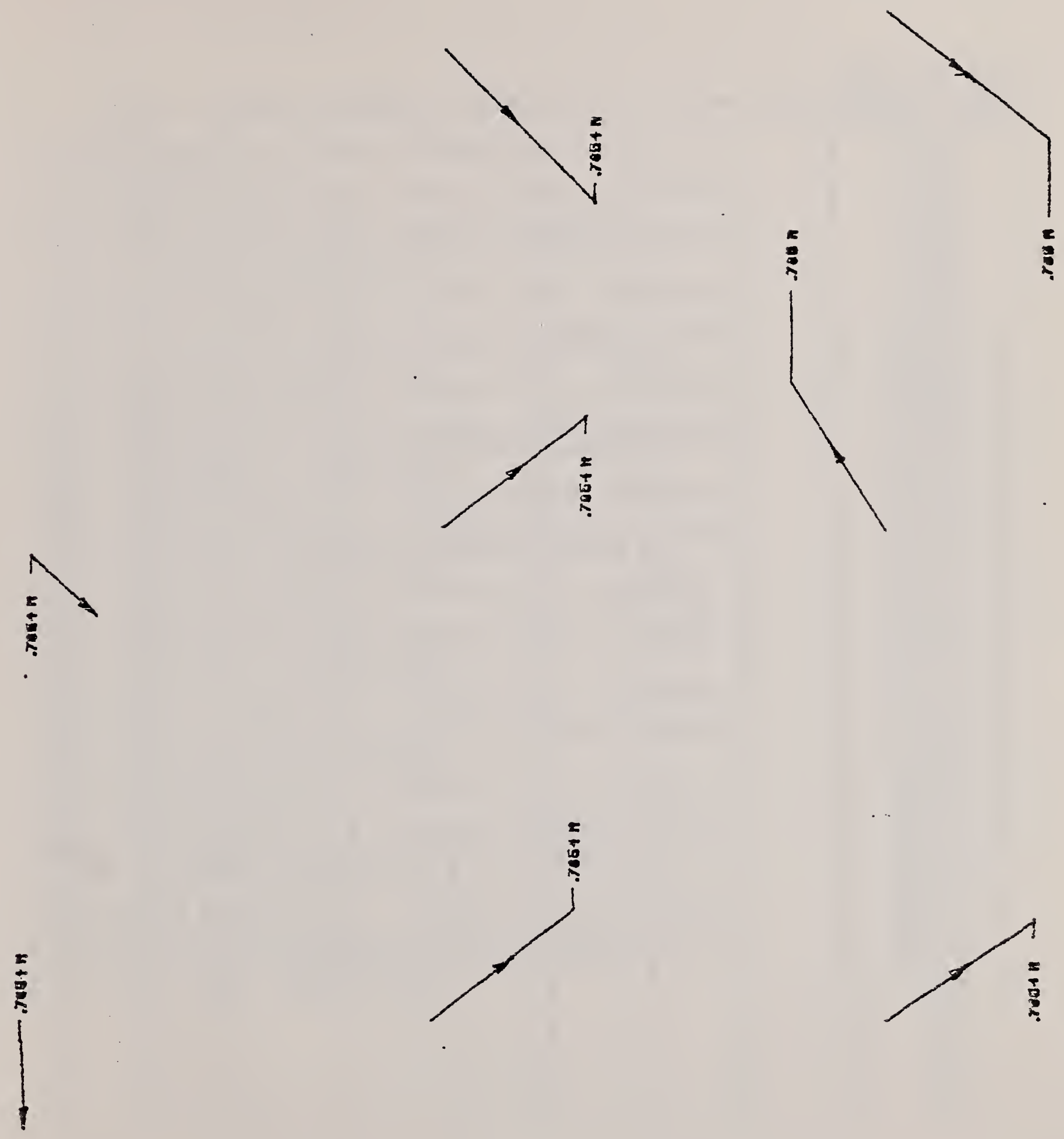
RDIA TEST CASE I

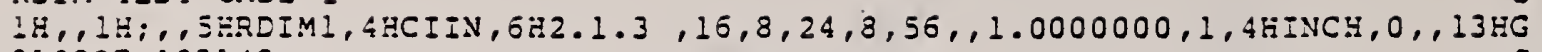

$810827.123146, \ldots$. ;

124

124

212

212

214

214

222

$2: 2$

212

214

214

222

222

212

212

214

214

222

222

212

212

214

214

222

222

?? 2

212

214

214

222

212

$2: 2$

$2 \div 4$

214

222

$2 z 2$

$2 i 2$

212

214

214

222

222

212

$2 \div 2$

214

222

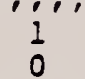

3

1
0
1

1
2
1
2
1
2
1
1
1
2
1
2
1
1
1
2
1
2
1
1
1
2
1
2
1
1
1
2
1
2
1
1
1
2
1
2
1
1
1
2
1
2
1
1
1
2
1
2
1

22

$$
\begin{aligned}
& 1 \\
& 0 \\
& 1
\end{aligned}
$$$$
1
$$$$
1
$$$$
\text { 10 }
$$$$
\begin{aligned}
& 0 \\
& 1 \\
& 0
\end{aligned}
$$$$
\begin{aligned}
& 0 \\
& 1 \\
& 0
\end{aligned}
$$$$
\begin{aligned}
& 1 \\
& 1 \\
& 0
\end{aligned}
$$$$
\begin{aligned}
& 0 \\
& 1 \\
& 0 \\
& 1
\end{aligned}
$$$$
\begin{aligned}
& 1 \\
& 1 \\
& 0
\end{aligned}
$$$$
1
$$$$
\frac{1}{0}
$$$$
\begin{array}{r}
0 \\
1 \\
0
\end{array}
$$$$
\begin{aligned}
& 1 \\
& 0 \\
& 1 \\
& 0
\end{aligned}
$$$$
0
$$$$
\begin{aligned}
& 1 \\
& 0 \\
& 1
\end{aligned}
$$$$
\begin{aligned}
& 0 \\
& 1 \\
& 0 \\
& 1 \\
& 0 \\
& 1 \\
& 0 \\
& 1 \\
& 0 \\
& 1 \\
& 0 \\
& 1 \\
& 0 \\
& 1 \\
& 0 \\
& 1 \\
& 0 \\
& 1 \\
& 0 \\
& 1 \\
& 0 \\
& 1
\end{aligned}
$$

0
0
0
0
0
3
0
0
0
0
0
3
0
0
0
0
0
3
0
0
0
0
0
3
0
0
0
0
0
3
0
0
0
0
0
3
0
0
0
0
0
3
0
0
0
0
0
3
0

00

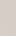

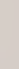$$
\begin{array}{r}
5 \\
7 \\
0 \\
8 \\
0 \\
10
\end{array}
$$

12

13

0
15

0
-17

0
18

0
20

0

23

0

25

27

28

30

32

32
33

33
0

35

0

37

0
38

0
40

42

.

$\begin{array}{lllll}0 & 0 & 0 & 0 & D \\ 0 & 0 & 1 & 1 & 0 \\ 0 & D \\ 0 & 0 & & & 0\end{array}$

$\begin{array}{lllll}0 & 0 & 1 & 1 & 0\end{array}$

$\begin{array}{lllll}0 & 0 & 0 & 1 & D \\ 0 & 0 & 0\end{array}$

$\begin{array}{lllll}0 & 0 & 1 & 1 & D\end{array}$

$\begin{array}{lllll}0 & 0 & 1 & 1 & 0 D\end{array}$

$\begin{array}{llllll}0 & 0 & 0 & 1 & D\end{array}$

$\begin{array}{llllll}0 & 0 & 1 & 1 & D\end{array}$

$\begin{array}{llll}0 & 0 & 1 & D \\ 0 & 0 & 1 & 1\end{array}$

$\begin{array}{lllll}0 & 0 & 1 & 1 & D\end{array}$

$\begin{array}{llllllll}0 & 0 & 0 & 1 & 0\end{array}$

$\begin{array}{llllll}0 & 0 & 1 & 1 & 0 D\end{array}$

$\begin{array}{llllllll}0 & 0 & 1 & 1 & D \\ 0 & 0 & 1 & 1 & 0\end{array}$

$\begin{array}{lllllllllll}0 & 0 & 1 & 1 & D \\ 0 & 0 & 0 & 1 & D\end{array}$

$\begin{array}{lll}0 & 0 & 0\end{array}$

$\begin{array}{lllll}0 & 0 & 1 & 1 & D \\ 0 & & & & 0 D\end{array}$

$\begin{array}{lllll}0 & 0 & 1 & 1 & 0\end{array}$

$\begin{array}{lllll}0 & 0 & 0 & 1 & D\end{array}$

$\begin{array}{lllll}0 & 0 & 1 & 1 & 0\end{array}$

$\begin{array}{lllllll}0 & 0 & 1 & 1 & 0\end{array}$

$\begin{array}{lllll}0 & 0 & 1 & 1 & 0\end{array}$

$\begin{array}{lllll}0 & 0 & 0 & 1 & D\end{array}$

$\begin{array}{llll}0 & 0 & 1 & 1\end{array}$

$0,1, O D$

$\begin{array}{lll}0 & 0 & 0 \\ 0 & 0 & 1\end{array}$

$00011 D$

$\begin{array}{lllll}0 & 0 & 1 & 1 & 0\end{array}$

$0 \quad 011$ OD

0.00 .10

47

48
40

\section{$3.14-4$}


222

$$
0
$$

0

1

0

$124,1.0000000,0.0,0.0,0.0,0.0,1.0000000,0.0,0.0,0.0,0.0$, $1.0000000,0.0,0,0$;

$212,1,7, .3185600, .0654000,1,1.5707963,0.0,0,0,2.1867423$, $5.1707363,0.0,78.7554$ R, 0,0;

$214,3, .1500000, .0500000,0.0,1.6395237,5.6704168,1.1796277$, $5.2822447,1.9905422,5.2034359,2.1213422,5.2034359,0,0$; $222,3,5,1.1796277,6.2822447,0,0$;

$212,1,7, .3185600, .0654000,1,1.5707963,0.0,0,0,4.8446350$, $5.0808229,0.0,7 \mathrm{H} .7654 \mathrm{R}, 0,0$;

$214,3, .1500000, .0500000,0.0,5.2303839,5.5779562,4.7724924$, $6.1912851,5.5771151,5.1135225,5.4463148,5.1135225,0,0$; $222,9,11,4.7724924,6.1912851,0,0$;

$212,1,7, .3185600, .0654000,1,1.5707963,0.0,0,0,7.3218365$, $5.0108891,0.0,71.7654 \mathrm{R}, 0,0$;

$214,3, .1500000, .0500000,0.0,7.7043066,5.6335106,8.2402897$, $6.1799164,7.1256361,5.0435886,7.2564363,5.0435836,0,0$; $222,15,17,8.2402897,6.1799164,0,0$;

$232,1,7, .3185600, .0654000,1,1.5707963,0.0,0,0,1.1881292$, $1.6041441,0: 0,74.7654 \mathrm{R}, 0,0$;

$214,3, .1500000, .0500000,0.0,1.5900917,2.1570453,1.1795277$, $2.3030782,1.9206092,1.6368442,1.7898092,1.5358442,0,0$; $222,21,23,1.1796277,2.8030782,0,0$;

$212,1,7, .3185600, .0654000,1,1.5707963,0.0,0,0,1.2453539$, $9.4113960,0.0,7 \mu .7654 \mathrm{R}, 0,0$;

$214,2, .1500000, .0500000,0.0, .4092200,9.4117279,1.0491536$, $9.4440956,1.1799538,9.4440956,0,0$; $222,27,29,1.1736424,9.4503927,0,0$;

$212,1,7, .3185500, .0654000,1,1.5707963,0.0,0,0,3.8433030$, $9.33 \$ 4724,0.0,75.7654$ R, 0,0;

$214,2, .1500000, .0500000,0.0,4.1367135,8.8636198,4.5757833$, $9.3641720,4.4449830,9.3641720,0,0$;

$222,33,35,4.6414399,9.4390230,0,0$;

$212,1,5, .2466200, .0654000,1,1.5707963,0.0,0,0,6.5733256$, $3.4967549,0.0,5 \mathrm{H} .765 \mathrm{R}, 0,0$;

$214,3, .1500000, .0500000,0.0,5.3853970,3.2309401,4.7529535$, $2.7998343,5.8233256,3.5294549,6.4733257,3.5294549,0,0$; $222,39,41,4.7529533,2.7998343,0,0$;

$212,1,6, .2466200, .0654000,1,1.5707963,0.0,0,0,6.5733256$, $1.4967549,0.0,64.765$ R, 0,0 ;

$214,3, .1500000, .0500000,0.0,8.0509501,2.1821513,8.5029516$, $2.7998343,7.5733261,1.5294548,7.0317178,1.5294548,0,0$; $222,45,47,8.5029516,2.7998343,0,0$; IG

$2 D$ $50 ?$

42

\begin{tabular}{rr}
$0 D$ & $5 C$ \\
$1 P$ & 1 \\
$1 P$ & 2 \\
$3 P$ & 3 \\
$3 P$ & 4 \\
$5 P$ & 5 \\
$5 P$ & 6 \\
$7 P$ & 7 \\
$9 P$ & 8 \\
$9 P$ & 9 \\
$11 P$ & 10 \\
$11 P$ & 12 \\
$13 P$ & 12 \\
$15 P$ & 13 \\
$15 P$ & 14 \\
$17 P$ & 15 \\
$17 P$ & 16 \\
$19 P$ & 17 \\
$21 P$ & 18 \\
$21 P$ & 19 \\
$23 P$ & 20 \\
$23 P$ & 21 \\
$25 P$ & 22 \\
$27 P$ & 23 \\
$27 P$ & 24 \\
$29 P$ & 25 \\
$29 P$ & 26 \\
$31 P$ & 27 \\
$33 P$ & 28 \\
$33 P$ & 29 \\
$35 P$ & 30 \\
$35 P$ & 31 \\
$37 P$ & 32 \\
$39 P$ & 33 \\
$39 P$ & 34 \\
$41 P$ & 35 \\
$41 P$ & 36 \\
$43 P$ & 37 \\
$45 P$ & 38 \\
$45 P$ & 39 \\
$47 ?$ & 40 \\
$47 ?$ & 41 \\
$49 ?$ & 42 \\
\hline 5 & $\vdots$ \\
&
\end{tabular}




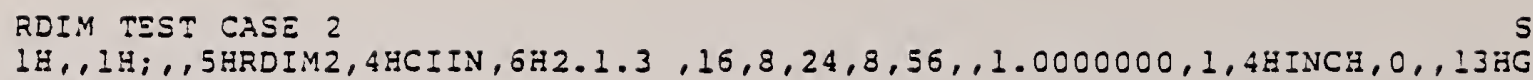
$810827.123219, \ldots$.;

$\begin{array}{lrl}124 & 1 & 1 \\ 124 & 0 & 0 \\ 212 & 3 & 1 \\ 212 & 0 & 0 \\ 214 & 5 & 1 \\ 214 & 0 & 0 \\ 222 & 7 & 1 \\ 222 & 0 & 0 \\ 212 & 8 & 1 \\ 212 & 0 & 0 \\ 214 & 10 & 1 \\ 214 & 0 & 0 \\ 222 & 12 & 1 \\ 222 & 0 & 0 \\ 212 & 13 & 1 \\ 212 & 0 & 0 \\ 214 & 15 & 1 \\ 214 & 0 & 0 \\ 222 & 17 & 1 \\ 222 & 0 & 0 \\ 212 & 18 & 1 \\ 212 & 0 & 0 \\ 214 & 20 & 1 \\ 214 & 0 & 0 \\ 222 & 22 & 1 \\ 222 & 0 & 0 \\ 212 & 23 & 1 \\ 212 & 0 & 0 \\ 214 & 25 & 1 \\ 214 & 0 & 0 \\ 222 & 27 & 1 \\ 222 & 0 & 0 \\ 212 & 28 & 1 \\ 212 & 0 & 0 \\ 214 & 30 & 1 \\ 214 & 0 & 0 \\ 222 & 32 & 1 \\ 222 & 0 & 0 \\ 212 & 33 & 1 \\ 212 & 0 & 0 \\ 214 & 35 & 1 \\ 214 & 0 & 0 \\ 222 & 37 & 1 \\ 222 & 0 & 0 \\ 212 & 38 & 1 \\ 212 & 0 & 0 \\ 214 & 40 & 1 \\ 214 & 0 & 0 \\ 222 & 42 & 1 \\ & & \end{array}$

1
2
1
2
1
2
1
1
1
2
1
2
1
1
1
2
1
2
1
1
1
2
1
2
1
1
1
2
1
2
1
1
1
2
1
2
1
1
1
2
1
2
1
1
1
2
1
2
1

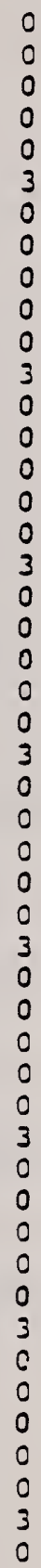

0

0

$\begin{array}{lllll}0 & 0 & 0 & 0 & 0\end{array}$

$\begin{array}{lllll}0 & 0 & 1 & 1 & 0\end{array}$

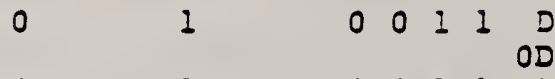

西

$\begin{array}{llllll}0 & 0 & 1 & 1 & D \\ 0 & 0 & & 0 & 0\end{array}$

000010

\begin{tabular}{lllll}
0 & 0 & 1 & 1 & $0 D$ \\
\hline
\end{tabular}

0

$\begin{array}{lllll}0 & 0 & 1 & 1 & 0\end{array}$

$\begin{array}{lllll}0 & 0 & 0 & 1 & D\end{array}$

$0 \quad 011$ OD

$0 \quad 011$ OD

- 01 OD

$\begin{array}{lllll}0 & 0 & 0 & 1 & 0\end{array}$

$\begin{array}{llllllll}0 & 0 & 1 & 1 & D\end{array}$

$\begin{array}{llllll}0 & 0 & 1 & 1 & O D\end{array}$

$\begin{array}{lllll}0 & 0 & 1 & 1 & 0 \\ 0 & 0 & 0 & 1\end{array}$

$\begin{array}{lllll}0 & 0 & 0 & 1 & D\end{array}$

$\begin{array}{lllll}0 & 0 & 1 & 1 & D\end{array}$

25

00110

1

$\begin{array}{lllll}0 & 0 & 0 & 1 & 0\end{array}$

30

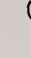

$\begin{array}{lllllllll}0 & 0 & 0 & 0 & 0 & 0\end{array}$

$\begin{array}{lllll}0 & 0 & 1 & 1 & D\end{array}$

32

01

$0 \begin{array}{lllll}0 & 0 & 1 & 1 & 0\end{array}$

$\begin{array}{ll}0 & 1 \\ 0 & 1\end{array}$

$\begin{array}{lllll}0 & 0 & 0 & 1 & 0 D\end{array}$

35

$\begin{array}{ll}0 & 1 \\ 0 & 1 \\ 0 & 1 \\ 0 & 1 \\ 0 & 1 \\ 0 & 1\end{array}$

$\begin{array}{ll}0 & 1 \\ 0 & 1 \\ 0 & 1 \\ 0 & 1 \\ 0 & 1 \\ 0 & 1\end{array}$

O OD

36.

$\begin{array}{lllll}0 & 0 & 1 & 1 & 0\end{array}$

0 O I I D

38

39

0

3

$\begin{array}{lllllll}0 & 0 & 0 & 1 & 0\end{array}$

$4:$

42

44

$\begin{array}{llllll}0 & 0 & 1 & 1 & 0\end{array}$

45

45

00110

48 
222

0

1

0

$124, .9973390, .0360059,-.0633904,0.0,-.0729025, .4925772$, -.8672098,0.0,0.0,.8695235,.4938914,0.0,0,0;

$212,1,7, .3185600, .0654000,1,1.5707963,0.0,0,0,2.1867423$, $5.1707363,0.0,79.7654 \quad R, 0,0 ;$

$214,3, .1500000, .0500000,0.0,1.6395237,5.6704168,1.1796277$, $6.2822447,1.9905422,5.2034359,2.1213422,5.2034359,0,0$;

$222,3,5,1.1796277,6.2822447,0,0$;

$212,1,7, .3185600, .0654000,1,1.5707953,0.0,0,0,4.8446350$, .

$5.0808229,0.0,7 \mathrm{R} .7654 \mathrm{R}, 0,0$;

$214,3, .1500000, .0500000,0.0,5.2303839,5.5779562,4.7724924$, $6.1912851,5.5771151,5.1135225,5.4463148,5.1135225,0,0$;

$222,9,11,4.7724924,6.1912851,0,0$;

$212,1,7, .3185600, .0554000,1,1.5707963,0.0,0,0,7.3218365$,

$5.0108891,0.0,7 \mathrm{.} .7654 \mathrm{R}, 0,0$;

$214,3, .1500000, .0500000,0.0,7.7043066,5.6335106,8.2402897$, $6.1799164,7.1256361,5.0435886,7.2554363,5.0435886,0,0 ;$

$222,15,17,8.2402897 ; 6.1799164,0,0$;

$212,1,7, .3185600, .0654000,1,1.570 .7963,0.0,0,0,1.1881292$,

$1.6041 \$ 41,0.0,79.7654 \mathrm{R}, 0,0$;

$2: 4,3, .1500000, .0500000,0.0,1.5900917,2.1570468,1.1796277$, $2.8030782,1.9205092,1.6368442,1.7898092,1.6368442,0,0$;

$222,21,23,1.1796277,2.8030782,0,0$;

$212,1,7, .3185600, .0654000,1,1.5707963,0.0,0,0,1.2453539$,

$9.4113960,0.0,7 \mathrm{H} .7654 \mathrm{R}, 0,0$;

$214,2, .1500000, .0500000,0.0, .4092200,9.4117279,1.0491536$,

$9.4440956,1.1799538,9.4440956,0,0$;

$222,27,20,1.1736424,9.4503927,0,0$;

$212,1,7, .3185600, .0654000,1,1.5707963,0.0,0,0,3.8433030$,

$9.3314724,0.0,7 \% .7654$ R, 0, 0:

$214,2, .1500000,00500000,0.0,4.1367135,8.8636198,4.5757833$,

$9.3641720,4.4449830,9.3641720,0,0$;

$222,33,35,4.6414399,9.4390230,0,0$;

$212,1,6, .2466200, .0654000,1,1.5707953,0.0,0,0,6.5733256$,

$3.4967549,0.0,67.765 \mathrm{R}, 0,0$;

$214,3, .1500000, .0500000,0.0,5.3853970,3.2309401,4.7529535$, $2.7998343,5.8233256,3.5294549,5.4733257,3.5294549,0,0$;

$222,39,41,4.7529535,2.7998343,0,0$ :

$212,1,6, .2466200, .0554000,1,1.5707963,0.0,0,0,6.5733256$, $1.4967549,0.0,67.765 \quad R, 0,0$;

$214,3, .1500000, .0500000,0.0,8.0509501,2.1821518,8.5029516$, $2.7998343 ., 7.5733261,1.5294548,7.0317178,1.5294548,0,0$;

$222,45,47,8.5029516,2.7998343,0,0$;

$\mathrm{s}$

IG

$2 D$

SOP

42

\begin{tabular}{rr} 
QD & 50 \\
$1 P$ & 1 \\
$1 P$ & 2 \\
$3 P$ & 3 \\
$3 P$ & 4 \\
$5 P$ & 5 \\
$5 P$ & 6 \\
$7 P$ & 7 \\
$9 P$ & 8 \\
$9 P$ & 9 \\
$11 P$ & 10 \\
$11 P$ & 11 \\
$13 P$ & 12 \\
$15 P$ & 13 \\
$15 P$ & 14 \\
$17 P$ & 19 \\
$17 P$ & 16 \\
$19 P$ & 17 \\
$21 P$ & 18 \\
$21 P$ & 19 \\
$23 P$ & 20 \\
$23 P$ & 21 \\
$25 P$ & 22 \\
$27 P$ & 23 \\
$27 P$ & 24 \\
$29 P$ & 25 \\
$29 P$ & 25 \\
$31 P$ & 27 \\
$33 P$ & 28 \\
$33 P$ & 29 \\
$35 P$ & 30 \\
$35 P$ & 31 \\
$37 P$ & 32 \\
$39 P$ & 33 \\
$39 P$ & 34 \\
$41 P$ & 35 \\
$41 P$ & 36 \\
$43 P$ & 37 \\
$45 P$ & 38 \\
$45 P$ & 39 \\
$47 P$ & $4 C$ \\
$47 P$ & 49 \\
$49 P$ & 42 \\
$T$ & 3 \\
\hline &
\end{tabular}





\subsubsection{Leader}

The following conventions were followed:

- solid font

- arrowhead FORM=3

- $\quad Z$ displacement set to 0

Case 1 (Figure 3.3.15-1) tests the following:

- independent leaders with variable lengths

Subordinate leaders are also tested in the label and angular, diameter, linear, and radius dimension cases. 


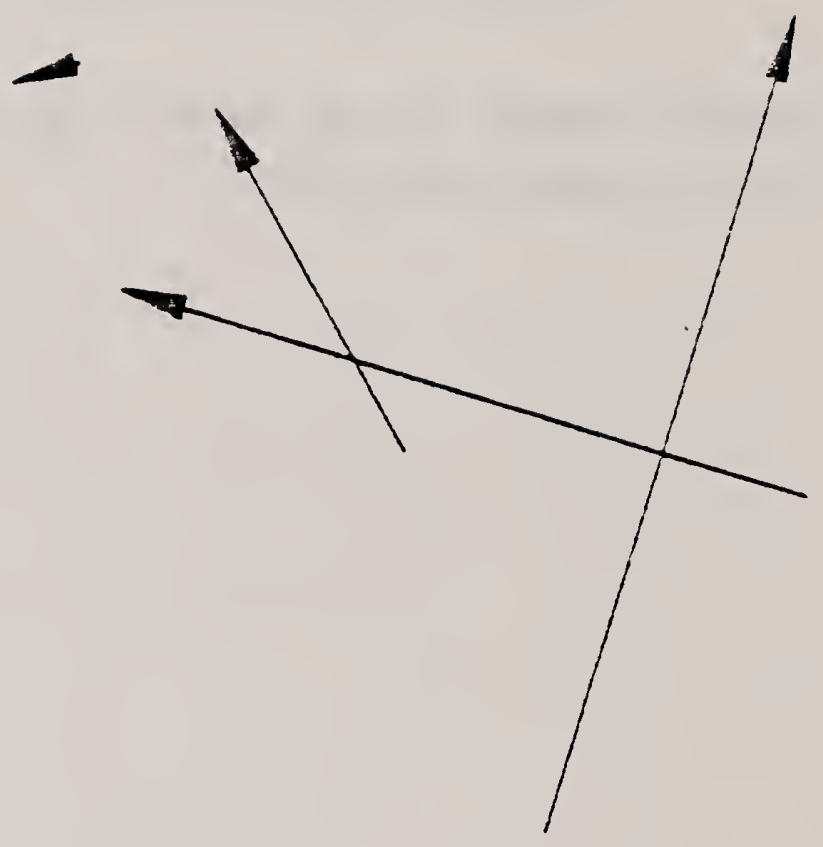

Figure 3.3.15-1 Leader Case 1 (actual part)

Case 1: Rotation $\left(0^{\circ}, 0^{\circ}, 0^{\circ}\right)$

IGES Test Deí.

D-282

$3.15-2$ 
EEADER TEST CASE 1

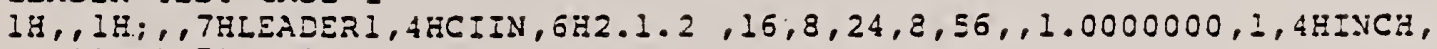

$0,, 13 \mathrm{H} 810714.085817, \ldots$ :

$\begin{array}{lclllll}124 & 1 & 1 & 1 & 0 & 0 & 0 \\ 124 & 0 & 0 & 2 & 0 & 0 & 0 \\ 214 & 3 & 1 & 1 & 0 & 0 & 1 \\ 214 & 0 & 0 & 2 & 3 & 0 & 0 \\ 214 & 5 & 1 & 1 & 0 & 0 & 1 \\ 214 & 0 & 0 & 2 & 3 & 0 & 0 \\ 214 & 7 & 1 & 1 & 0 & 0 & 1 \\ 214 & 0 & 0 & 2 & 3 & 0 & 0 \\ 214 & 9 & 1 & 1 & 0 & 0 & 1 \\ 214 & 0 & 0 & 2 & 3 & 0 & 0 \\ 214 & 11 & 1 & 1 & 0 & 0 & 1 \\ 214 & 0 & 0 & 2 & 3 & 0 & 0\end{array}$

$124,1.0000000,0.0,0.0,0.0,0.0,1.0000000,0.0,0.0,0.0,0.0$,

$1.0000000,0.0,0,0$ :

$214,2, .1600000, .1290000,0.0,1.5319998,2.3839998,2.1759996$,

$2.7039995,2.1759996,2.7039995,0,0$;

$214,2, .1600000, .1280000,0.0,2.8319998,4.2799997,3.0799994$,

$4.3520002,3.0799994,4.3520002,0,0 ;$

$214,2, .1600000, .1280000,0.0,3.5999994,4.1920004,4.3280001$; $2.3519997,4.3280001,2.9519997,0,0$;

$214,2, .1600000, .1280000,0.0,5.7760000,4.5679998,4.8959999$, $1.5599999,4.8959999,1.5599999,0,0 ;$

$214,2, .1600000, .1280000,0.0,3.2559996,3.5279994,5.3479996$, $2.8079996,5.8479996,2.8079996,0,0$;

s

16

20

$12 p$

12

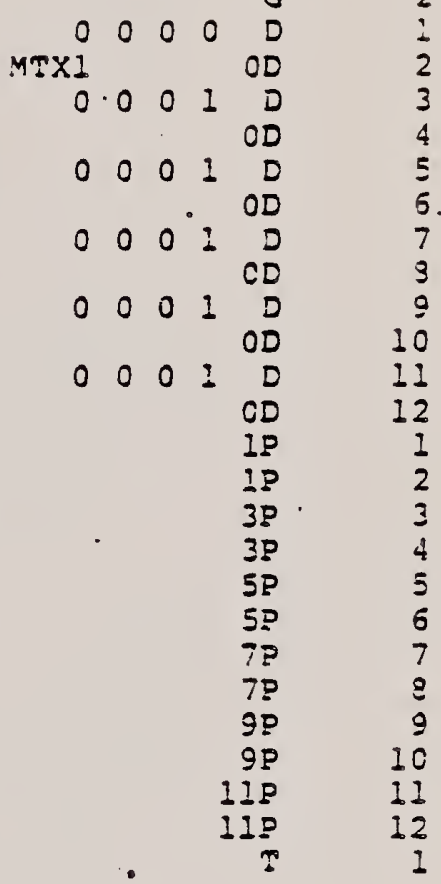


3.3.16 Section

Data case not available.

IGES Test Def.

D-282

$3.16-1$ 


\subsubsection{Witness Line}

The witness line contains one or more line segments associated with drafting entities (angular dimension and linear dimension). The test for witness lines is part of the tests for angular and linear dimensions.

The following conventions were followed:

- IF parameter set to 1

- N parameter set to 3

- solid font

- witness line is subordinate only 
3.3.18 Associativity Instance

Case 1 (Figure $3.3 .18-1$ ) tests the following:

- a single group composed of 10 points

Case 2 (Figure 3.3.18-2) tests the following:

- grouping (association) of line and point entities

- 7 levels of nesting

Each subtree in the figure represents a grouping with the level of the subtree representing the level of group nesting. 


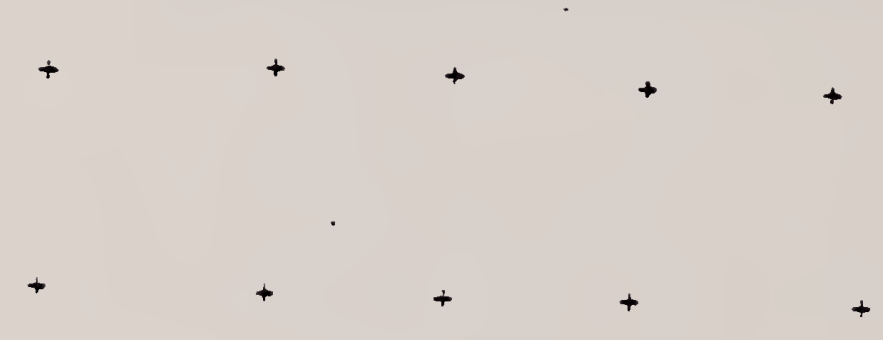

Figure 3.3.18-1 Associativity Instance Case 1 (actual part) Case 1: Rotation $\left(0^{\circ}, 0^{\circ}, 0^{\circ}\right)$ 


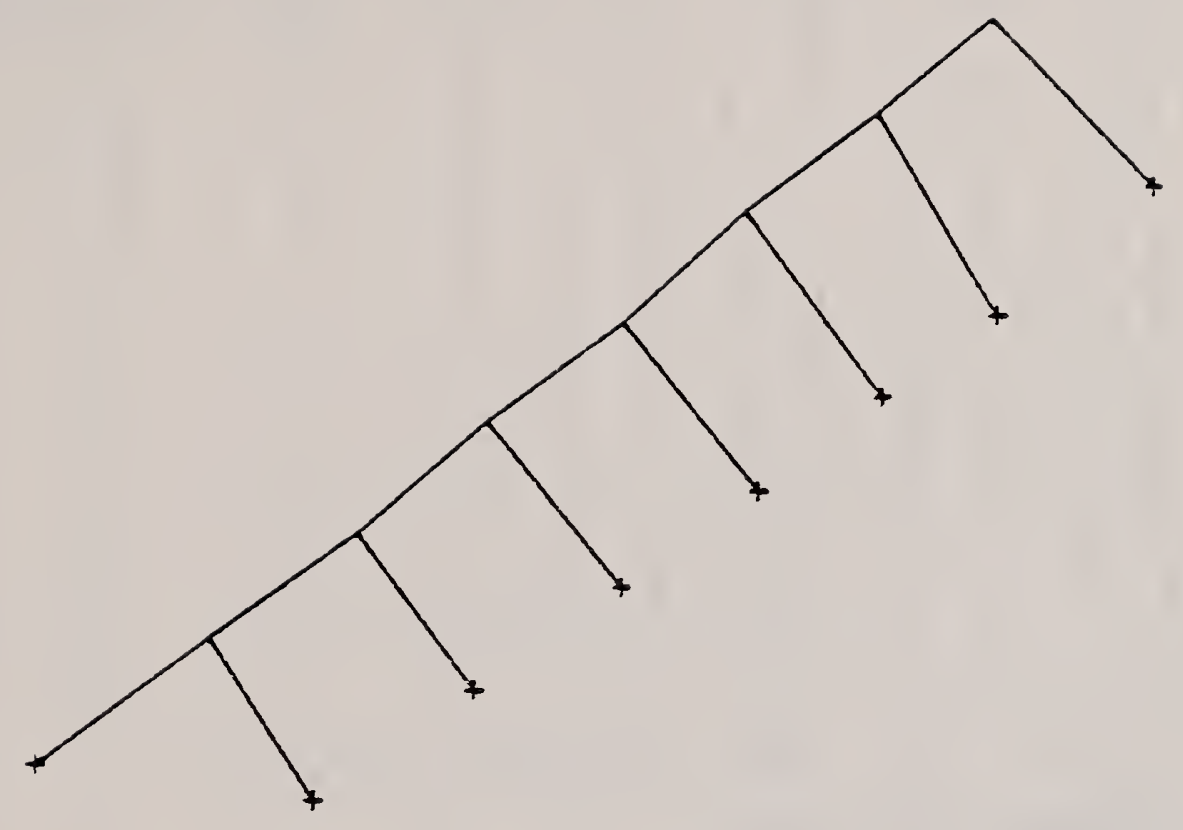

Figure 3.3.18-2 Associativity Instance Case 2 (actual part) Case 1: Rotation $\left(0^{\circ}, 0^{\circ}, 0^{\circ}\right)$ 


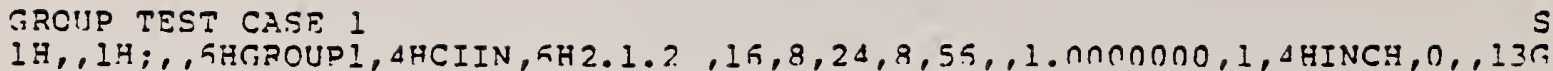
4810713.101823,., :

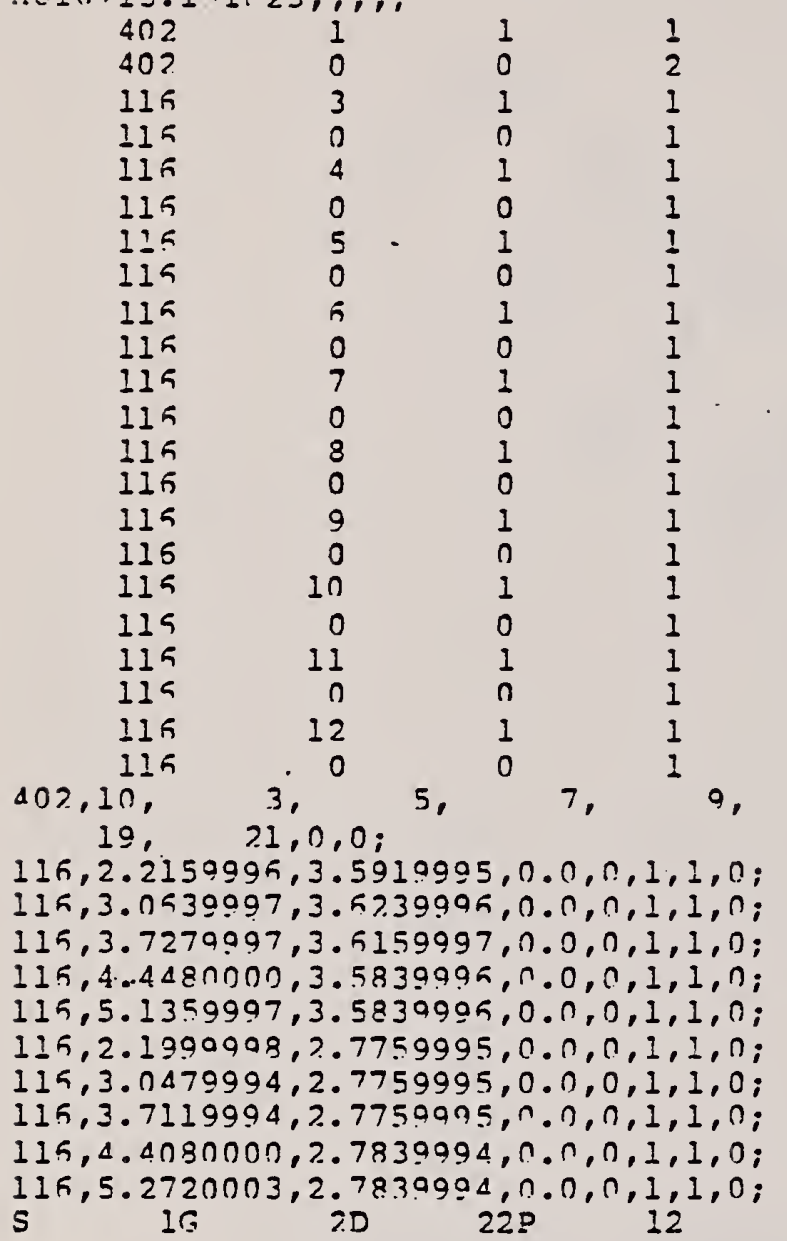

\begin{tabular}{|c|c|c|}
\hline$n$ & 0 & n \\
\hline 1 & $n$ & 0 \\
\hline 0 & $n$ & 0 \\
\hline 0 & 0 & $n$ \\
\hline ก & 0 & 0 \\
\hline 0 & 0 & 0 \\
\hline 0 & 0 & 0 \\
\hline 0 & 0 & 0 \\
\hline 0 & 0 & 0 \\
\hline 0 & 0 & 0 \\
\hline 0 & $n$ & ? \\
\hline 0 & 0 & 0 \\
\hline 0 & 0 & $n$ \\
\hline 0 & $n$ & 0 \\
\hline 0 & 0 & 0 \\
\hline 0 & 0 & $n$ \\
\hline 0 & n & 0 \\
\hline 0 & 0 & 0 \\
\hline 0 & 0 & n \\
\hline 0 & 0 & 0 \\
\hline 0 & 0 & n \\
\hline 0 & 0 & 0 \\
\hline 11, & 13, & 15, \\
\hline
\end{tabular}

$00 n 00$

$\begin{array}{lllll}0 & n & 1 & 0 & 0\end{array}$

$\begin{array}{llllll}0 & 0 & 1 & 0 & 0 & 1 D\end{array}$

$P \quad 2 D$

$\begin{array}{lllll}0 & \cap & 1 & \cap & D\end{array}$

P $3 D$

$\begin{array}{lllll}0 & \cap & 1 & 0 & D\end{array}$

P $4 \mathrm{D}$

$\begin{array}{lllll}0 & 0 & 1 & 0 & D\end{array}$

$P \quad 5 D$

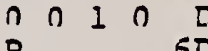

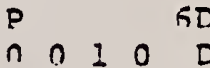

$\begin{array}{llllll}0 & 7 D\end{array}$

$\begin{array}{lllll}0 & 0 & 1 & 0 & 0\end{array}$

80

$\begin{array}{lllll}n & 0 & 1 & \cap & D\end{array}$

$\begin{array}{llllllll}0 & 0 & 0 & 0\end{array}$

$\begin{array}{lllll}0 & 0 & 1 & 0 & 0\end{array}$

17,

p

$\begin{array}{rr}0 & 0 \\ 10 n & 21 \\ 1 P & 27 \\ 1 P & 1 \\ 3 P & 3 \\ 5 D & 4 \\ 7 P & 5 \\ 9 P & 5 \\ 11 P & 7 \\ 13 P & 8 \\ 15 P & 9 \\ 17 P & 10 \\ 19 P & 11 \\ 21 P & 12 \\ 7 & 1 \\ & \end{array}$


GROUP TEST CASE 2

IH, IH, , , 5HGROUP2, 4 HCIIN , 6H2.1.2, 16,8,24,8,56, ,1.0000000,1, 4HINCH, 0, , 23G

H810714.101934, ...

$\begin{array}{lrll}402 & 1 & 1 & 1 \\ 402 & 0 & 0 & 1 \\ 110 & 2 & 1 & 1 \\ 116 & 0 & 0 & 1 \\ 110 & 3 & 1 & 1 \\ 110 & 0 & 0 & 1 \\ 110 & 4 & 1 & 1 \\ 110 & 0 & 0 & 1 \\ 402 & 5 & 1 & 1 \\ 402 & 0 & 0 & 1 \\ 116 & 6 & 1 & 1 \\ 116 & 0 & 0 & 1 \\ 110 & 1 & 1 & 1 \\ 110 & 0 & 0 & 1 \\ 110 & 8 & 1 & 1 \\ 110 & 0 & 0 & 1 \\ 402 & 9 & 1 & 1 \\ 102 & 0 & 0 & 1 \\ 116 & 10 & 1 & 1 \\ 116 & 0 & 0 & 1 \\ 110 & 11 & 1 & 1 \\ 110 & 0 & 0 & 1 \\ 110 & 12 & 1 & 1 \\ 110 & 0 & 0 & 1 \\ 402 & 13 & 1 & 1 \\ 402 & 0 & 0 & 1 \\ 116 & 14 & 1 & 1 \\ 116 & 0 & 0 & 1 \\ 110 & 15 & 1 & 1 \\ 110 & 0 & 0 & 1 \\ 110 & 16 & 1 & 1 \\ 110 & 0 & 0 & 1 \\ 402 & 17 & 1 & 1 \\ 402 & 0 & 0 & 1 \\ 116 & 18 & 1 & 1 \\ 116 & 0 & 0 & 1 \\ 110 & 19 & 1 & 1 \\ 110 & 0 & 0 & 1 \\ 110 & 20 & 1 & 1 \\ 110 & 0 & 0 & 1 \\ 402 & 21 & 1 & 1 \\ 402 & 0 & 0 & 1 \\ 116 & 22 & 1 & 1 \\ 116 & 0 & 0 & 1 \\ 110 & 23 & 1 & 1 \\ 110 & 0 & 0 & 1 \\ 110 & 24 & 1 & 1 \\ 110 & 0 & 0 & 1 \\ 402 & 25 & 1 & 1 \\ & & & \end{array}$

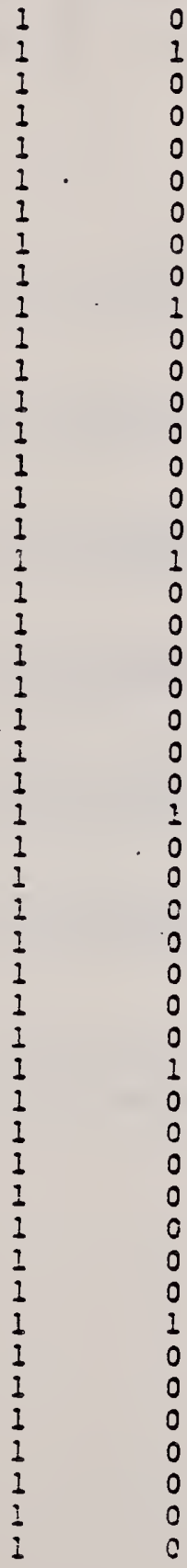

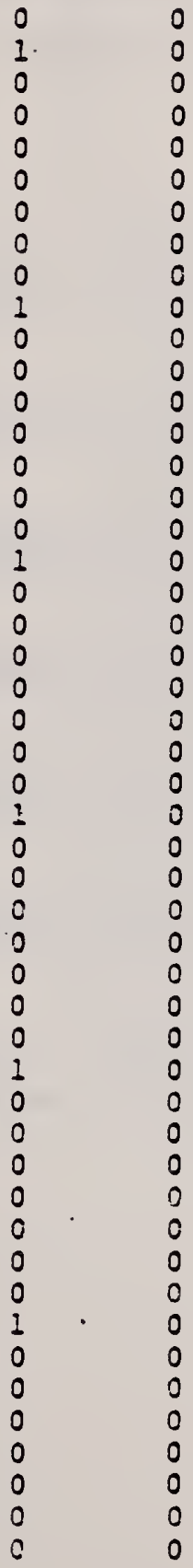

$\begin{array}{llllllllll}0 & 0 & 0 & 0 & 0\end{array}$

$\begin{array}{llllllll}0 & 0 & 1 & 0 & 0 & 0\end{array}$

2010

II 10 OD

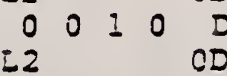

$\begin{array}{llllllllll}0 & 0 & 1 & 0 & 0\end{array}$

$\begin{array}{lllll}0 & 0 & 1 & 0 & D\end{array}$

$\begin{array}{llllll}0 & 0 & 1 & 0 & 0 \\ 0 & 0 & 0 & 0 & 0\end{array}$

L3

$\begin{array}{lllll}0 & 0 & 1 & 0 & D\end{array}$

L4

0010

$\begin{array}{lllllll}0 & 0 & 1 & 0 & 0 D\end{array}$

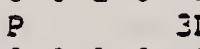

$\begin{array}{rrrrr}0 & 0 & 1 & 0 & 0 \\ -5 & & & & 0 \\ 0 & 0 & 0\end{array}$

001100

$\left[\begin{array}{llll}6 & 0 & 0\end{array}\right.$

$\begin{array}{lllll}0 & 0 & 1 & 0 & 0\end{array}$

$0 \begin{array}{lllll}0 & 0 & 1 & 0 & 0\end{array}$

3 4D

$00=0 \quad D$

L7

$\begin{array}{lllll}0 & 0 & 1 & 0 & 0 D\end{array}$

L8

0010

$\begin{array}{llllll}0 & 0 & 1 & 0 & 0 \\ 0 & & & & 5 \\ 0 & 0 & 0 & 0 & 0 & 0\end{array}$

0 o : 0

L9

LIO

$0 \quad 0 \quad 1 \quad 0 \quad 0$

00100

$\begin{array}{rrrrrr}0 & 0 & 1 & 0 & \\ 0 & 0 & 1 & 0 & 0 \\ 111 & & & & 0 D \\ 0 & 0 & 1 & 0 & 0 \\ 12 & & & & 0 \\ 0 & 0 & 1 & 0 & 0\end{array}$ 


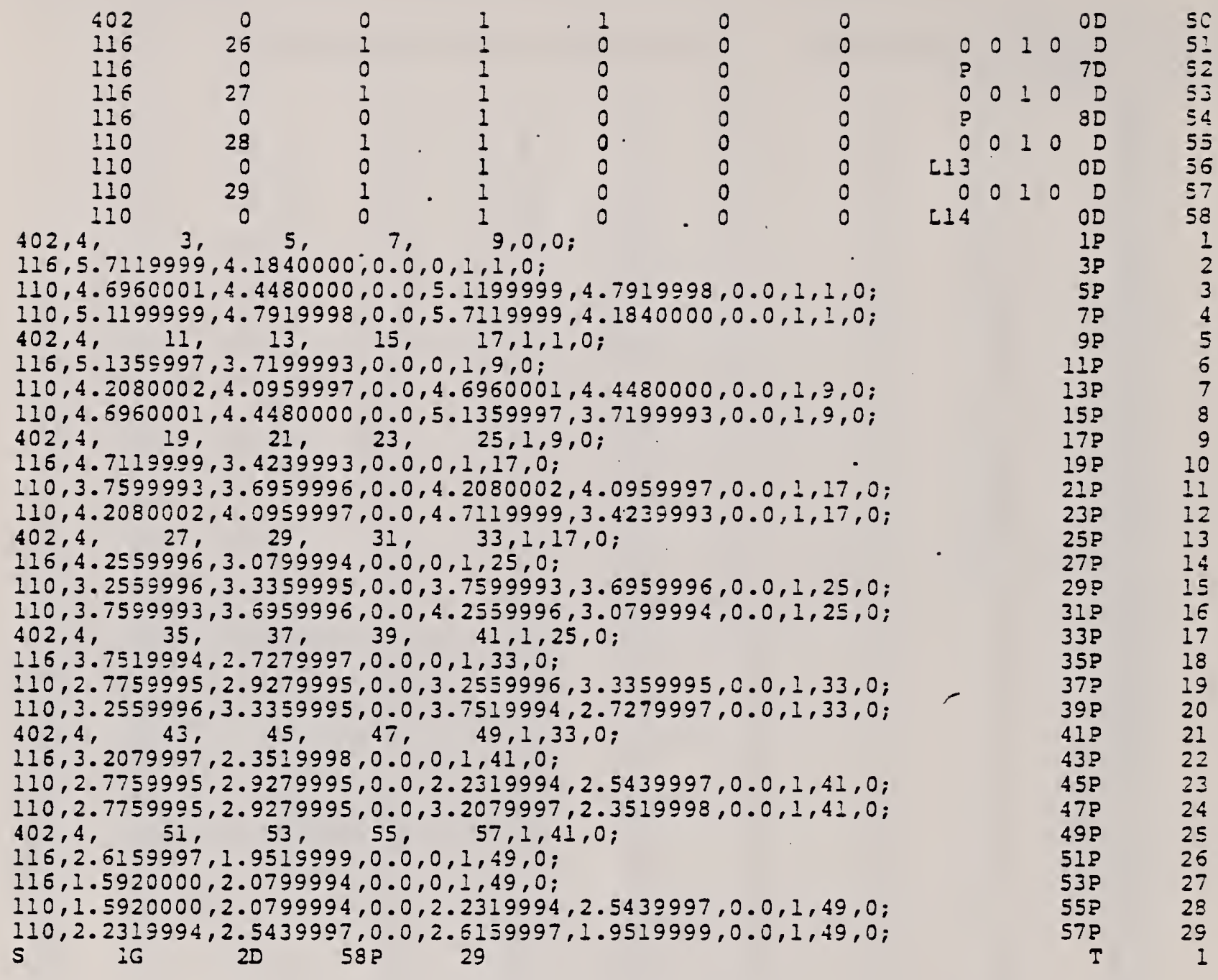




\subsubsection{Line Fonts}

The four line fonts of solid, dashed, phantom, and centerline are tested in various entity tests incuding those for lines, arcs, and conics. The fonts are defined qualitatively only and no data concerning exact dash and gap lengths is included. Thus the display of these fonts on different systems may appear differently. Examples of the different fonts are shown in Figure 3.3.19-1. 


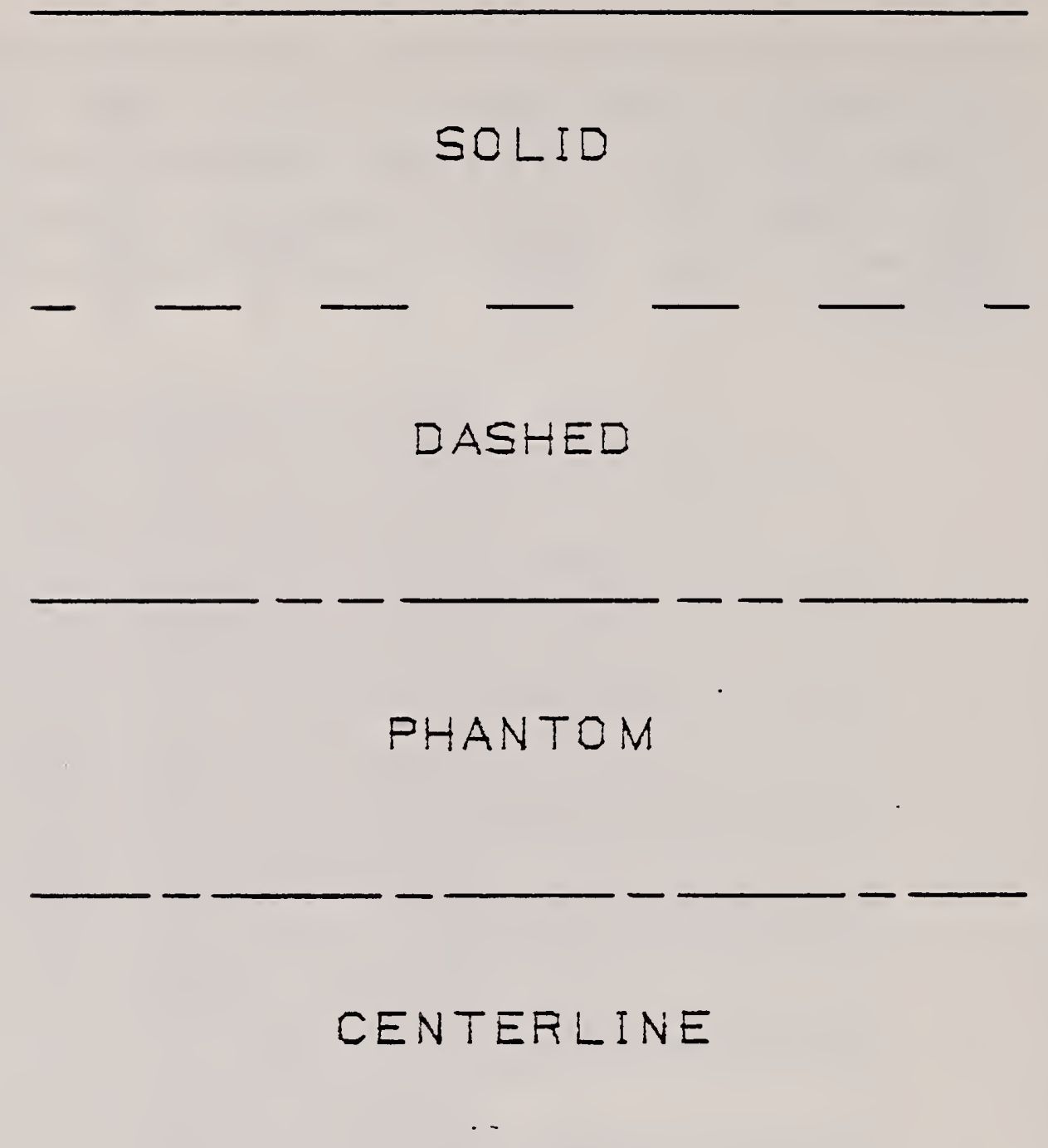




\subsubsection{Leyels}

Case 1 (Figures 3.3.20-1 and 3.3.20-2) tests the following:

- levels 0-15 contain one point on each level (point label/subscript corresponds to the level it resides on) 


$$
\begin{aligned}
& \text { LEVEL } 12 \text { LEVEL I3 LEVEL IS LEVEL IS } \\
& \text { LEVEL } 8 \text { LEVEL } 9 \text { LEVEL } 10 \text { LEVEL II } \\
& \text { LEVEL } 4 \text { LEVEL } 5 \text { LEVEL } 6 \text { LEVEL } 7 \\
& \text { LEVEL O LEVEL I LEVEL } 2 \text { LEVEL } 3
\end{aligned}
$$

Figure 3.3.20-1 Level Case 1 (with annotation and all levels visible) Case 1: Rotation $\left(0^{\circ}, 0^{\circ}, 0^{\circ}\right)$ 

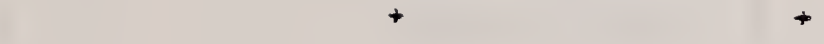
LEVEL TEST CASE I

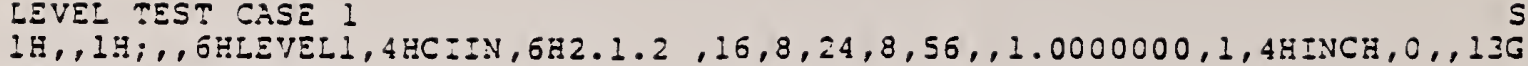
แ810713.191719,,,

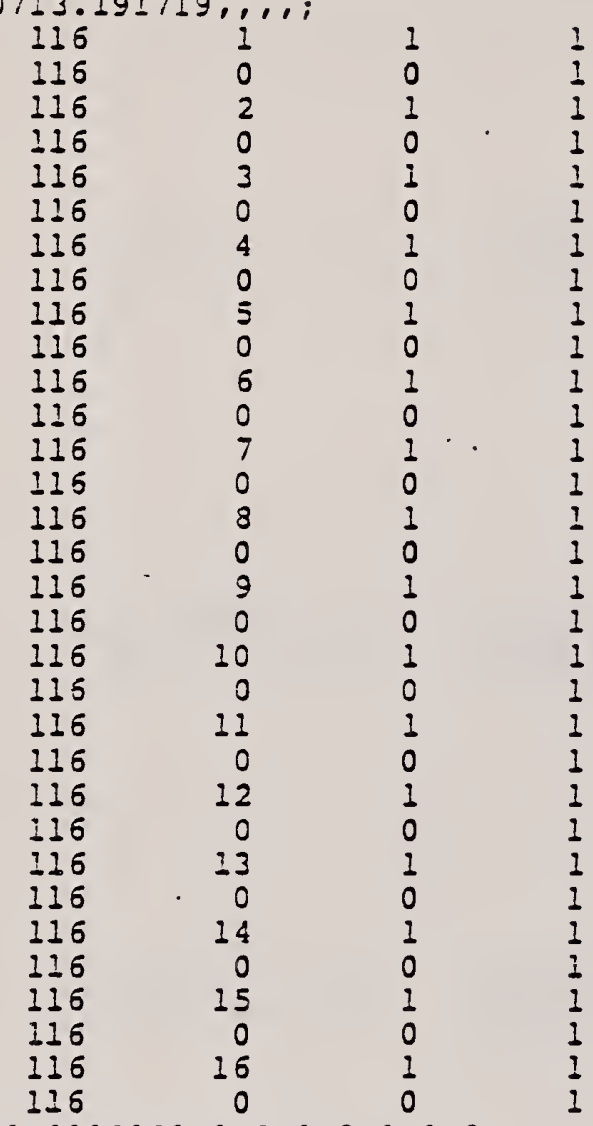

$116,1.0000000,0.0,0.0,0,0,0$;

$\begin{array}{cc}1 & 1 \\ 1 & 0 \\ 1 & 2 \\ 1 & 0 \\ 1 & 3 \\ 1 & 0 \\ 1 & 4 \\ 1 & 0 \\ 1 & 5 \\ 1 & 0 \\ 1 & 6 \\ 1 & 0 \\ 1 & 7 \\ 1 & 0 \\ 1 & 8 \\ 1 & 0 \\ 1 & 9 \\ 1 & 0 \\ 1 & 10 \\ 1 & 0 \\ 1 & 11 \\ 1 & 0 \\ 1 & 12 \\ 1 & 0 \\ 1 & 13 \\ 1 & 0 \\ 1 & 14 \\ 1 & 0 \\ 1 & 15 \\ 1 & 0 \\ 1 & 0 \\ 1 & 0\end{array}$

$116,2.0000000,0.0,0.0,0,0,0$;

$116,3.0000000,0.0,0.0,0,0,0$;

$116,0.0,1.0000000,0.0,0,0,0$;

$116,1.0000000,1.0000000,0.0,0,0,0$;

$116,2.0000000,1.0000000,0.0,0,0,0$;

$116,3.0000000,1.0000000,0.0,0,0,0$;

$116,0.0,2.0000000,0.0,0,0,0$;

$116,1.0000000,2.0000000,0.0,0,0,0$;

$116,2.0000000,2.0000000,0.0,0,0,0$;

$116,3.0000000,2.0000000,0.0,0,0,0$;

$116,0.0,3.0000000,0.0,0,0,0$;

$116,1.0000000,3.0000000,0.0,0,0,0$;

$116,2.0000000,3.0000000,0.0,0,0,0$;

$116,3.0000000,3.0000000,0.0,0,0,0$;

$116,0.0,0.0,0.0,0,0,0$;

s

16

$2 D$

$32 \mathrm{P}$

16

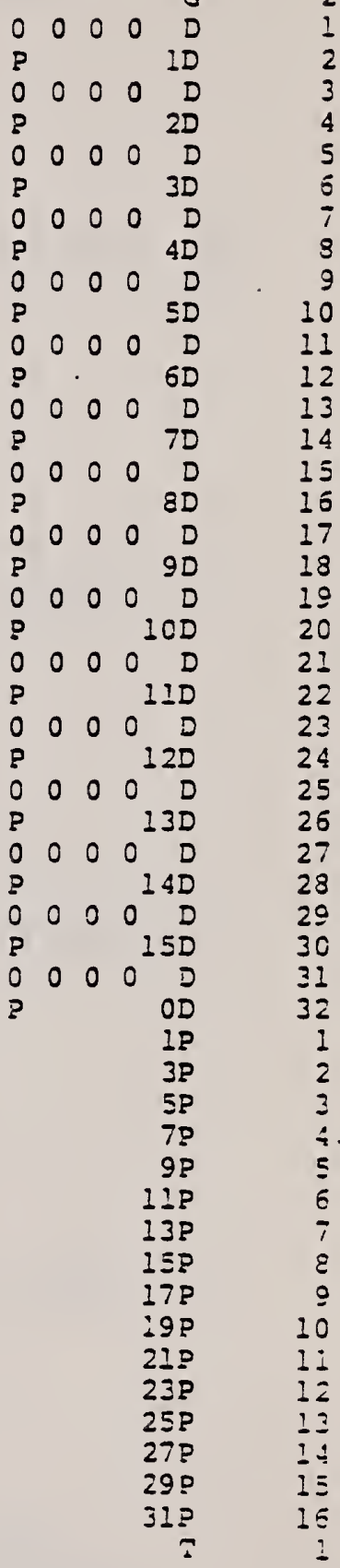




\subsubsection{BLANK Status}

Case 1 (Figure 3.3.21-1) tests the following:

$$
\begin{aligned}
&-\quad \text { Blanked geometry } \\
&- \text { points } \\
&- \text { lines } \\
&- \text { conics }
\end{aligned}
$$

- Blanked annotation

- general note

- general label

- linear dimension

- radius dimension

- diameter dimension

- angular dimension 


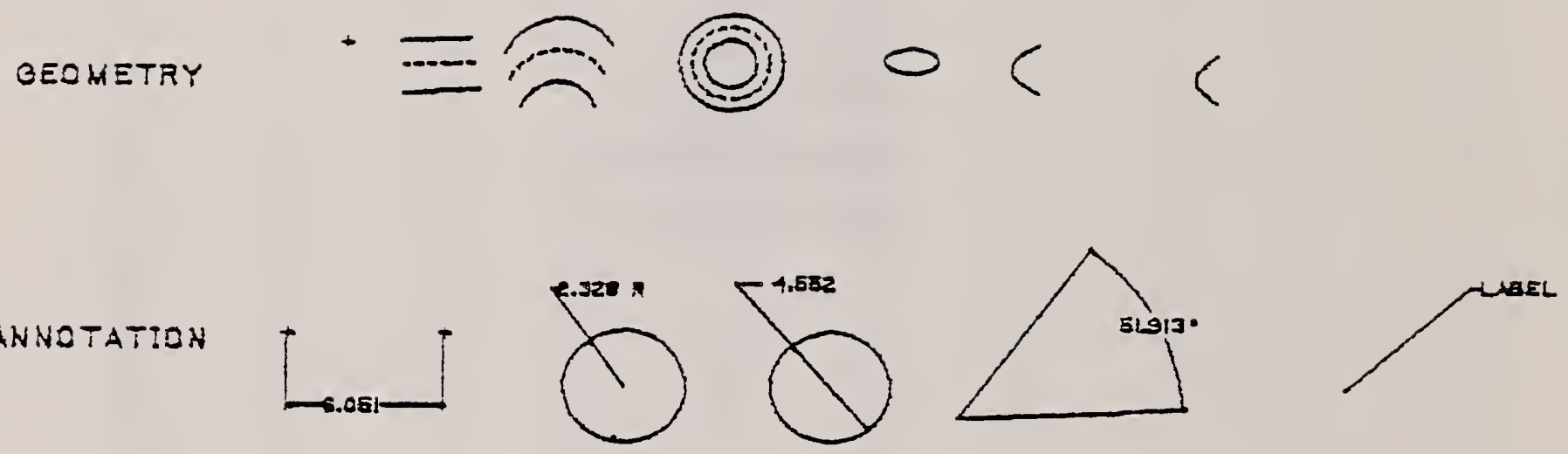

Figure 3.3.21-1 Blank Status Case 1 (actual entities are blanked) Case 1: Rotation $\left.0^{\circ}, 0^{\circ}, 0^{\circ}\right)$ 
BLANK TES? CASE 1

$1 \mathrm{H}, 1 \mathrm{i} ;,, 6 \mathrm{HBLANKI}, 4 \mathrm{HCIIN}, 6 \mathrm{H} 2.1 .3,16,8,24,8,56,1,0000000,1,4 \mathrm{HINCH}, 0,13 \mathrm{G}$ 1810827.122534,., ,

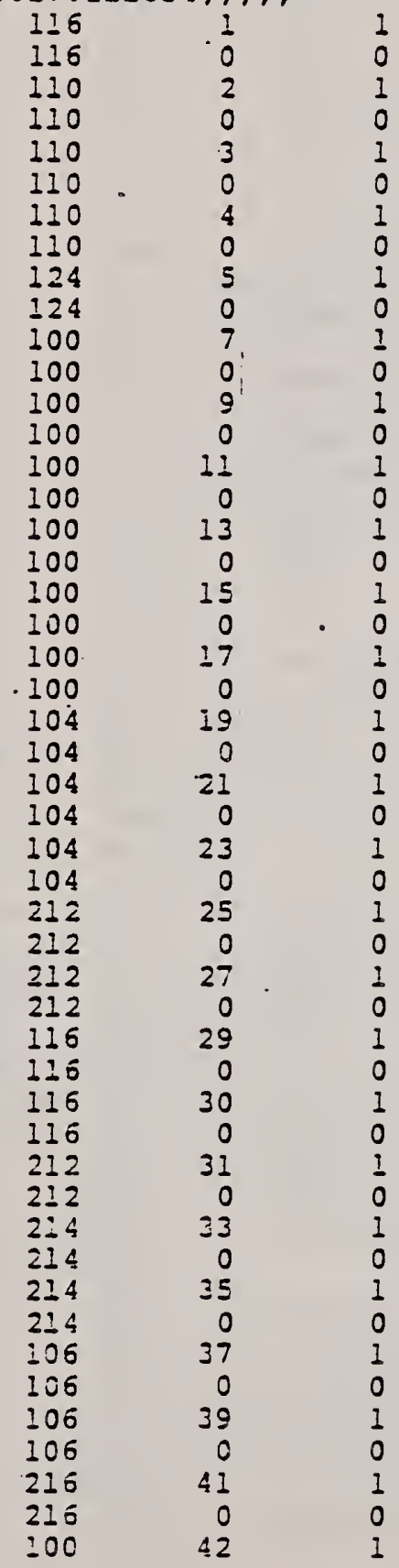

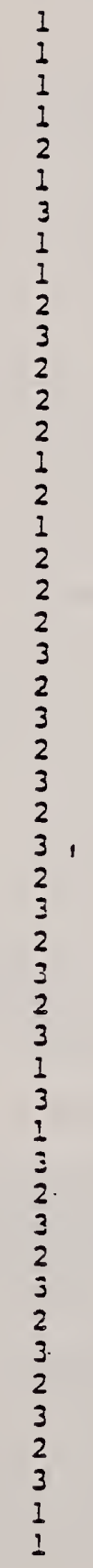
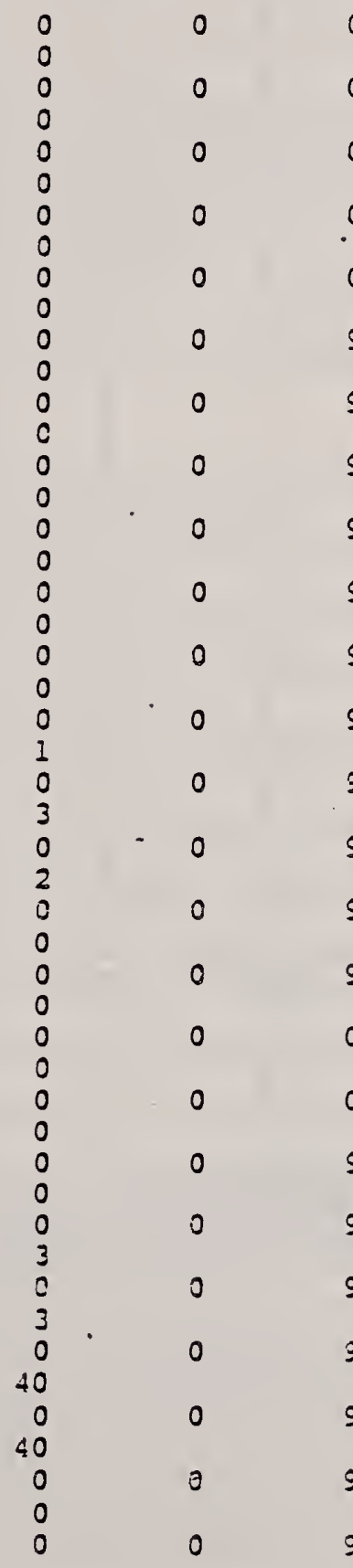

$\begin{array}{lllll}0 & 1 & 0 & 0 & 0\end{array}$

$\therefore$ iD

$\begin{array}{llllr}0 & 1 & 0 & 0 & 0\end{array}$

[I 1 OD

$\begin{array}{lllll}0 & 1 & 0 & 0 & D \\ 2 & & & & O D\end{array}$

$\begin{array}{lllll}0 & 1 & 0 & 0 & 0\end{array}$

$\left[\begin{array}{lllll}0 & 0 & 0 & 0\end{array}\right.$

$\begin{array}{lllll}0 & 0 & 0 & 0 & D \\ 0 & & & 0 & 0\end{array}$

$\begin{array}{lllll}0 & 1 & 0 & 0 & D\end{array}$

A1 OD

$\begin{array}{lllll}0 & 1 & 0 & 0 & 0\end{array}$

$\begin{array}{rllll}A 2 & & & & O D \\ 0 & 1 & 0 & 0 & 0\end{array}$

A3 OD

$\begin{array}{lllll}0 & 1 & 0 & 0 & 0\end{array}$

$C 1,1000$

C2 $\quad O D$

$\begin{array}{lllllll}0 & 0 & 1 & 0 & 0 & D & 21 \\ & C 3 & & & O D & 22\end{array}$

$\begin{array}{lllllll}0 & 0 & 1 & 0 & 0 & 0 & 23\end{array}$

3 CLPI 0100000

PRB I OD 16 $\begin{array}{llllll}0 & 1 & 0 & 0 & D & 27\end{array}$ EYPI OD 28 $\begin{array}{llllll}0 & 1 & 0 & 1 & D & 29\end{array}$ TXI OD 30 $\begin{array}{rrrrrr}0 & 1 & 0 & 1 & 0 & 31 \\ T \times 2 & & & O D & 32\end{array}$ $\begin{array}{llllllll}0 & 1 & 0 & 0 & D & 33\end{array}$ $\begin{array}{lllll}2 & 20 & 34\end{array}$ $\begin{array}{lllllll}0 & 1 & 0 & 0 & 0 & 35\end{array}$ $\begin{array}{llllllll}0 & & & & 3 D & 36 \\ 0 & 0 & 1 & 1 & 0 & 37\end{array}$ OD 38 0111035 $011: 0 \quad 41$ $001100 \quad 42$ 001 OD 4 OD 11 Q $0 \perp 010$ द $\begin{array}{llllrr}0 & 1 & 0 & 0 & 0 & 4 E\end{array}$ 


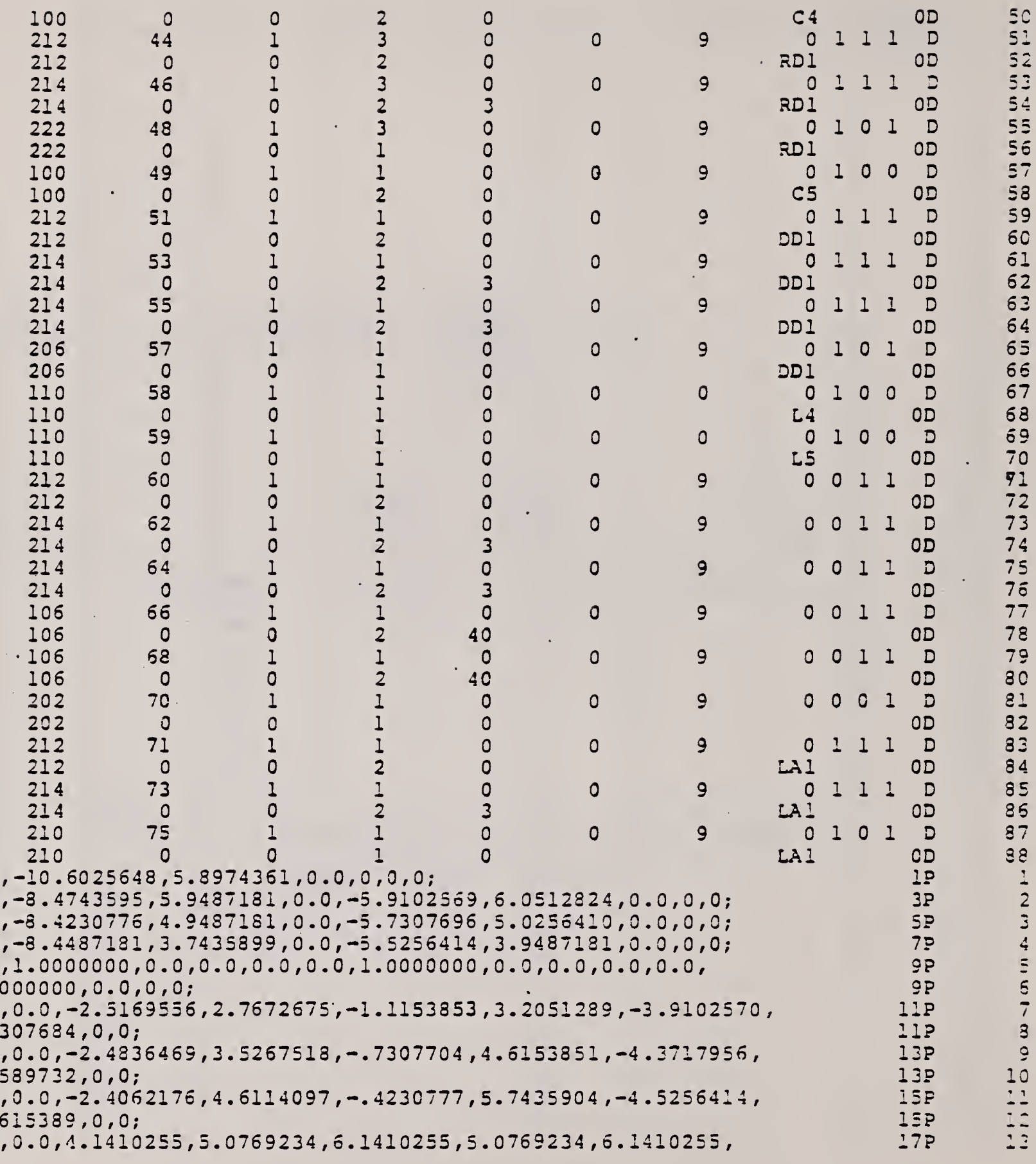


$5.0769218,0,0$;

$100,0.0,4.1410255,5.0759234,5.5410255,5.0769234,5.5410253$, $5.0769222,0,0$;

$100,0.0,4.1410255,5.0769234,5.1410255,5.0769234,5.1410255$,

$5.0769225,0,0$;

$104,1.0000000, .1430511 E-05,4.0000000,-22.2051351,-41.4359137$, $229.5753583,0.0,12.1025639,5.1794868,12.1025639,5.1794863,0,0$;

$104, .4706543 E-28,-.1372100 E-13,1.0000000,-1.0000000,-9.7435904$, $38.6331063,0.0,15.9487181,3.8717952,15.9487181,5.8717952,0,0$; $104, .2500000,0.0,-1.0000000,-10.0128202,3.9230776,79.3512408$, $0.0,22.8540588,3.4615381,22.8540668,5.4615378,0,0$;

$212,1,8,6.9750005, .7500001,1,1.5707963,0.0,0,0,-19.5641041$, $4.1025643,0.0,8$ KGEOMETRY, 0,0;

$212,1,10,8.1750006, .7500001,1,1.5707963,0.0,0,0,-20.0256424$,

$-6.9743590,0.0,10$ HANNOTATION, 0,0 ;

$116,-12.8974361,-5.3589745,0.0,0,0,0$;

$125,-5.8461547,-5.3076921,0.0,0,0,0$;

$212,1,5,2.0500000, .5000000,1,1.5707963,0.0,0,0,-11.4615393$,

$-9.5897436,0.0,536.051,0,0$;

$214,1, .1500000, .0500000,0.0,-12.8974361,-9.3397436,-11.5615396$, $-9.3397435,0,0$;

$214, i, .1500000, .0500000,0.0,-5.8461547,-9.3397436,-9.2215395$,

$-9.3397436,0,0$;

$106,1,3,0.0,-12.8974361,-5.4527245,-12.8974361,-6.4527245$,

$-12.8974361,-9.4647436,0,0$;

$106,1,3,0.0,-5.8461547,-5.4014421,-6.8461547,-5.4014421$,

$-6.8461547,-9.4647436,0,0$;

$215,37,39,41,43,45,0,0$;

$100,0.0, .0568982,-8.4683762,2.3846251,-8.4683752,2.3846251$,

$-8.4683780,0,0$;

$21<, 1,7,3.1500000, .5000000,1,1.5707963,0.0,0,0,-2.3846159$,

$-4.6665655,0.0,752.328 \mathrm{R}, 0,0$;

$214,3, .1500000, .0500000,0.0,-1.2475615,-0.5405025, .0568982$, $-8.4683762,-2.6846156,-4.4166665,-2.3846159,-4.4166665,0,0$; $222,51,53, .0568982,-8.4683762,0,0$

$100,0.0,3.0008917,-8.5078821,10.2770360,-8.5078821,10.2770360$, $-8.5078839,0,0$

$212,1,5,2.2500000, .5000000,1,1.5707963,0.0,0,0,5.8717942$,

$-4.4102564,0.0,5: 4.552,0,0$;

$214,3, .1500000, .0500000,0.0,6.5423788,-6.7505034,8.0010023$,

$-3.5078582,4.3717942,-4.1602564,5.3717942,-4.1602564,0,0$;

$214,1, .1500000, .0500000,0.0,9.4596258,-10.2552131,8.0010023$,

$-3.5078583,0,0$;

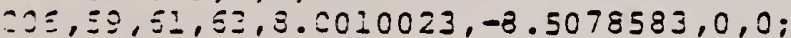

$110,19.2051277,-9.4871798,0.0,12.9487171,-9.7435894,0.0,0,0$;

:- $0.12 .9487171,-9.7435894,0.0,16.8974361,-4.2564101,0.0,0,0$;

$212,1, \sigma, 2.4500000, .5000000,1,1.5707963,0.0,0,0,19.1538467$,

$-5 . .025539,0.0,6151.913,0,0 ;$

$214.1, .1500000, .0500000,0.0,21.6179576,-9.3882931,20.9768944$. $-5.4526159,0,0$;

$=\{, \pm, \ldots 300000,00500000,0.0,18.0167206,-2.7010416,20.3860303$,

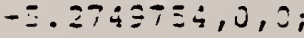


$106,1,3,0.0,19.2987991,-9.4833408,19.2987991,-9.4833408$, $21.7428628,-9.3831745,0,0$;

$106,1,3,0.0,16.9521960,-4.1803153,16.9521960,-4.1803153$,

$214,2, .1500000, .0500000,0.0,27.8572350,-8.5579443,32.6091995$,

$-4.2073960,32.9091988,-4.2073960,0,0 ;$

$210,83,1,85,0,0$; 


\subsubsection{Labels/Subscripts}

Case 1 (Figures $3.3 .22-1$ and $3.3 .22-2$ ) tests the following

- Points with label/subscripts with the following conditions:

. - Label/subscripts with one through six characters

- Label/subscripts with all alphabetic characters

- Label/subscripts with all numeric characters

Other test cases also contain entities with label/subscripts. 


\begin{tabular}{|c|c|c|c|c|c|}
\hline $\begin{array}{l}\text { LAEEL } \\
\text { ONLY. }\end{array}$ & \multicolumn{2}{|c|}{$\begin{array}{l}\text { SUESCRIFT } \\
\text { ONLY }\end{array}$} & & \multicolumn{2}{|c|}{$=L$ EOTH } \\
\hline A & 1 & + & + & $P$ & 11111 \\
\hline$A \equiv$ & 11 & + & + & $P T$ & 1111 \\
\hline$A E C$ & 111 & + & + & $P N T$ & 111 \\
\hline$A E C D$ & 1111 & + & + & FONT & 11 \\
\hline$A B C D E$ & 11111 & + & + & POINT & 1 \\
\hline$A D C D E F$ & 111111 & + & + & HIIAEC & \\
\hline
\end{tabular}

Figure 3.3.22-1 Label/Subscipt Case 1 (with annotation)

Case 1: Rotation $\left(0^{\circ}, 0^{\circ}, 0^{\circ}\right)$ 


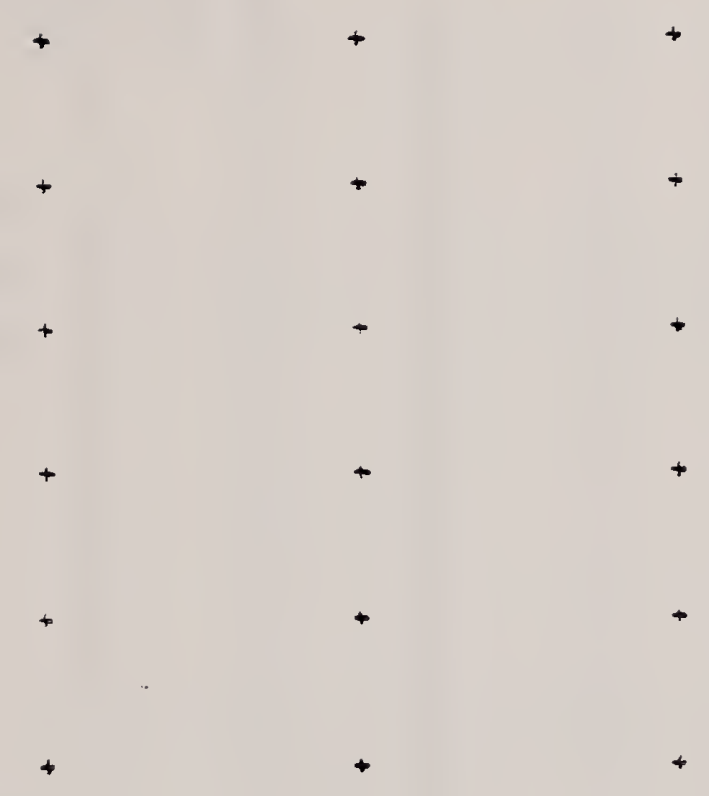

Figure 3.3.22-2 Label/Sucscript Case 1 (actual part) 


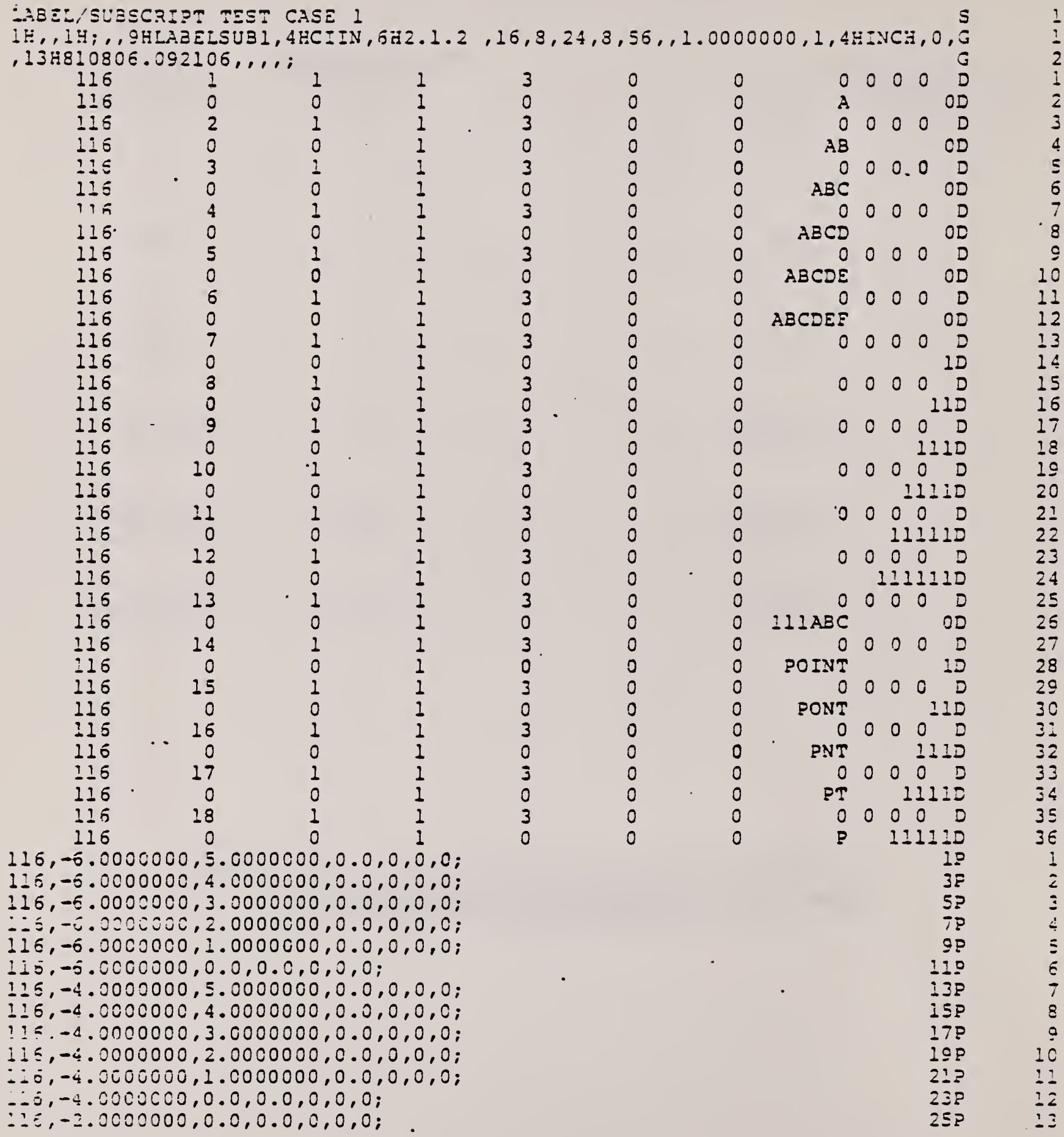


$116,-2.0000000,1.0000000,0.0,0,0,0$; $116,-2.0000000,2.0000000,0.0,0,0,0$; $115,-2.0000000,3.0000000,0.0,0,0,0$; $116,-2.0000000,4.0000000,0.0,0,0,0$; $116,-2.0000000 ; 5.0000000,0.0,0,0,0$; 



\section{REFERENCES}

A. Y14.26M-1981

American National Standard Engineering Drawing and Related Documentation Practices (sections 2 through 4 of this document were derived from the Initial Graphics Exchange Specification Version 1.0.)

B. Boeing Document Geometric Data Base File and Entity Format Standard D6-42705-210

C. 79-1847 Boeing's CAD/CAM Integrated Information Network AIAA Aircraft System and Technology Meeting, August 1979 
(a) 
NBS.114A (REV. 2-8C)

U.S. DEFT. OF COMM.

BIBLIOGRAPHIC DATA

SHEET (See in struction s)

1. PUBLICATION OR
REPORT NO.
NBSIR $83-2704$ (USAF)

2. Performing Organ. Report Nof 3. Publication Date

September 1983

4. TITLE ANO SUBTITLE

Initial Graphics Exchange Specification Test Library, Version 1.3.

5. AUTHOR(S) Bradford Smith, NBS, IGES Test, Evaluate \& Support Committee, Michael Liewaid, The Boeing Company

6. PERFORMING ORGANIZATION (If joint or other than NBS, see instructions)

NATIONAL BUREAU OF STANDARDS

DEPARTMENT OF COMMERCE

YASHINGTON, D.C. 20234

7. Contract Grant No.

FY 1457-82-N5024

8. Type of Report \& Period Covered

9. SPONSORING ORGANIZATION NAME AND COMPLETE AOORESS (Street, City, State, ZIP)

$9 / 30 / 81-2 / 28 / 83$

Air Force ICAM Program

Wright Patterson Air Force Base

Dayton, Ohio

10. SUPPLEMENTARY NOTES

$\square$ Document describes a computer program; SF-185, FIPS Software Summary, is attached.

11. ABSTRACT (A 200-ward or less factual summary of most significant information. If document includes a significant bibliography or literature survey. mention it here)

This document contains a library of bencinmark tests to be used to verify the interface capability possible with the Initial Graphics Exchange Specification (IGES) (Reference A). IGES provides a common data format to facilitate the exchange of data between different Computer Aided Design/Computer Aided Manufacturing (CAD/CAM) systems.

The test cases outlined in this document provide data for software modules which translate IGES format to and from the format of a particular CAD/CA.M system. The geometric and drafting entities contained in these parts comprise only a limited portion of all IGES entities and are intended to demonstrate some capabilities of an IGES translator on an individual entity basis. This set of tests does not constitute a test of compliance with any existing or proposed standardization of IGES. Subsequent tests with more complex parts should be made to insure the suitability of the processors for a user's production environment.

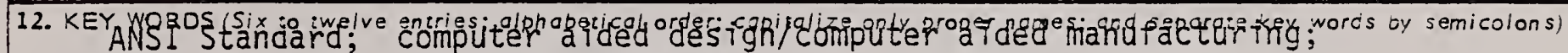
computer graphics; interface standard

13. AVAILABILITY

Unimited

For Official Distribution. Do Not Release to NTIS

Order From Superintendent of Documents, U.S. Government Printing Office, Washington, D.C. 20402.

X Order From National Technical information Service (NTiS), Springfield, VA. 22161
14. NO. OF

PRINTED PAGES

223

15. Price

$\$ 19.00$ 


